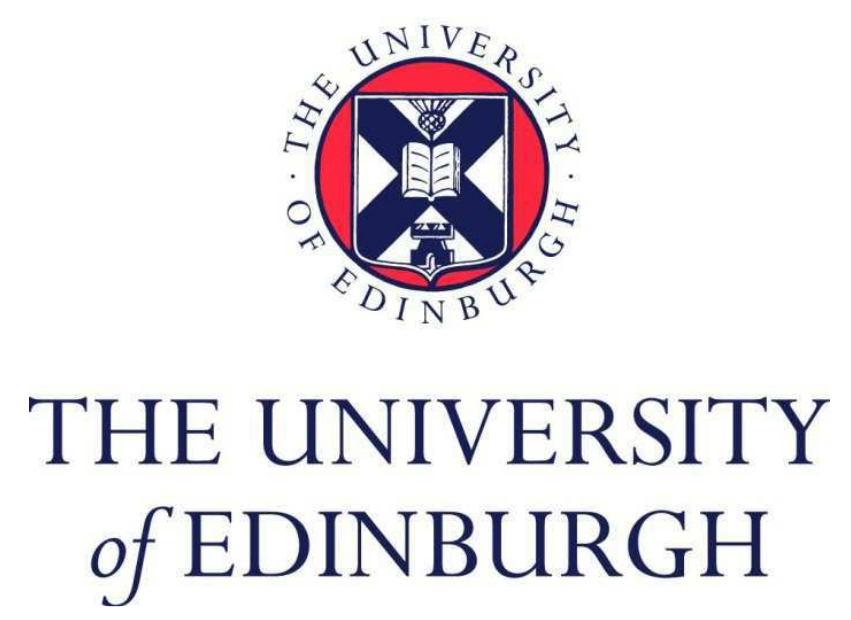

This thesis has been submitted in fulfilment of the requirements for a postgraduate degree (e.g. PhD, MPhil, DClinPsychol) at the University of Edinburgh. Please note the following terms and conditions of use:

This work is protected by copyright and other intellectual property rights, which are retained by the thesis author, unless otherwise stated.

A copy can be downloaded for personal non-commercial research or study, without prior permission or charge.

This thesis cannot be reproduced or quoted extensively from without first obtaining permission in writing from the author.

The content must not be changed in any way or sold commercially in any format or medium without the formal permission of the author.

When referring to this work, full bibliographic details including the author, title, awarding institution and date of the thesis must be given. 


\section{No missed opportunity: expanding sexual healthcare provision beyond current service delivery models}

Dr Rebecca Lily Heller BA MBChB MFSRH

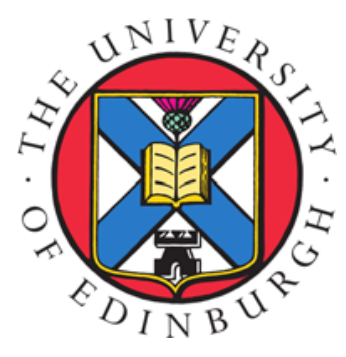

Doctor of Philosophy

The University of Edinburgh 


\section{Preface}

I declare that the thesis has been composed by myself and that the work has not been submitted for any other degree or professional qualification. I confirm that the work submitted is my own, except where work which has formed part of jointly-authored publications has been included. My contribution and those of the other authors to this work have been explicitly indicated below. I confirm that appropriate credit has been given within this thesis where reference has been made to the work of others.

\section{Chapters one, two and eight}

These chapters were composed solely by myself.

\section{Chapter three}

I designed and piloted the questionnaire, which was handed out by reception staff. I collated and analysed the results, and wrote the manuscript. SC and peer reviewers reviewed the manuscript.

\section{Chapters four and five}

SC and AG conceived the original idea for the research. I designed the research, organised pharmacist training and liaised with pharmacists throughout the research. $\mathrm{AJ}$ and myself recruited patients. I collected data from patients, undertook qualitative interviews with pharmacists and analysed both quantitative and qualitative data. SC reviewed parts of the chapters as a submission for publication, as did peer reviewers.

\section{Chapter six}

AG and SC conceived the original idea for the research. I collected data about pregnancy intervals. RB and NF handed out and collected questionnaires. I analysed the data and wrote the manuscript. SC and AG reviewed the manuscript, as did peer reviewers.

\section{Chapter seven}

$\mathrm{AG}$ and $\mathrm{SC}$ conceived the original research idea. I presented data to midwives and medical staff about insertion of IUC at section and trained medical staff. SC trained consultants. I liaised with medical staff in the early days of the service to answer questions. I saw all patients for six week follow up with AJ. AJ did the majority of 
telephone contact at three, six and 12 months. SC reviewed the parts of the chapter as a submission for publication, as did peer reviewers.

Signed:

Dr Rebecca Lily Heller BA MBChB MFSRH

\section{Publications resulting from this work}

Heller R, Johnstone A, Cameron ST. The feasibility of contraceptive injections at the community pharmacy. Eur J Contracept Reprod Health Care 2017 doi:10.1080/13625187.2017.1357808 [Epub ahead of print]

Heller R, Johnstone A, Cameron ST. Routine provision of intrauterine contraception at elective cesarean section in a national public health service: an observational study. Acta Obstet Gynecol Scand 2017;96(9):1144-1151.

Heller R, Cameron S, Briggs R, Forson, N, Glasier A. Author's response to comment on 'Postpartum contraception: a missed opportunity to prevent unintended pregnancy and short inter-pregnancy intervals.' J Fam Plan Reprod Health Care 2016;43(2)164-165.

Heller R, Cameron ST. Evaluating the attractiveness of the availability of injectable progestogen contraceptives at the community pharmacy setting in the United Kingdom. Int J Pharm Pract 2016;24(4):247-252.

Heller R, Cameron S, Briggs R, Forson, N, Glasier A. Postpartum contraception: a missed opportunity to prevent unintended pregnancy and short inter-pregnancy intervals. J Fam Plan Reprod Health Care 2016;42(2)93-98.

\section{International presentations}

An evaluation of postplacental insertion of intrauterine contraception (PPIUC) at elective caesarean section in the UK. International Federation of Professional Abortion and Contraception Associates (FIAPAC). Lisbon, Portugal. October 14-15 2016

An evaluation of postplacental insertion of intrauterine contraception at elective caesarean section. Royal College of Obstetricians and Gynaecologists (RCOG) World Congress. Birmingham, UK. June 20-22 2016 (Selected to be featured in Congress Closing Remarks) 


\section{Abstract}

Background: Despite a wide range of contraceptive options available in the United Kingdom, the unplanned pregnancy rate remains high. Contraceptive services are currently delivered by general practitioners, sexual health clinics and pharmacies, but there may be scope to expand the places that these are offered, and increase the options available within each service. Doing so could increase the uptake of contraceptive methods, particularly the most effective methods, and therefore reduce the unplanned pregnancy rate.

Aim and objectives: Research in this thesis aimed to investigate novel delivery models of contraception. The research had two main areas of focus. Firstly the capacity of the pharmacy to deliver regular contraception was examined, in the context of existing literature, and then through a pilot study. After that the expansion of contraception care to maternity services was investigated, first in the literature and then using an observational study.

Methods: In undertaking this thesis I used a variety of methods. Two patient surveys were employed to investigate patients' perspectives on proposed novel methods of contraceptive delivery. A pilot study investigated the feasibility and acceptability of delivery of the contraceptive injection at the pharmacy. Quantitative results about the numbers of injections given were collected, as were patient questionnaires.

Qualitative one-to-one interviews were conducted with participating pharmacists, these were recorded, transcribed and analysed.

An observational study was also undertaken to assess routine delivery of insertion of intra-uterine contraception at the time of caesarean section. Patients were seen at six weeks following insertion, and contacted by telephone at three, six and 12 months about satisfaction and continuation of the method.

Results: 220 women completed a questionnaire about attending the community pharmacy to receive a contraception injection. $33 \%$ of current non-users indicated that they would consider using this method if it was available at the pharmacy. 50 established users of the contraceptive injection participated in a pilot project receiving up to three injections from the community pharmacy. Only 48 injections of 
a possible 150 were delivered at the community pharmacy. Only 7 participants received all three injections at the pharmacy, and participants reported mixed experiences accessing the pharmacy. The practical obstacles around pharmacy engagement and the challenges of retaining participants were significant, and more research is necessary before proceeding with a randomised controlled trial. 250 women on a postnatal ward completed questionnaires about their pregnancy intentions. $96.7 \%$ were not planning a baby in the next year, but only $23.6 \%$ were planning on using the most effective methods of contraception. One in three respondents described themselves as likely to use either an implant or intra-uterine contraception if it could be inserted before they left the hospital.

In an observational study, 120/877 women opted to have intra-uterine contraception inserted at the time of caesarean section. Continuation rates at 12 months were $84.8 \%$ of those contacted, and $92.6 \%$ were either 'very' or 'fairly' happy with their contraception.

Conclusion: Although patients are receptive to contraception being delivered using novel service models, alternatives to current practice need careful investigation. Contraceptive injections at the community pharmacy are not necessarily more convenient for patients, and therefore may not increase uptake of this method. However, offering intrauterine contraception to patients at the time of caesarean section is highly acceptable to patients, and results in a substantial majority continuing this highly effective method.

Robust and careful research using a range of methods can help to identify which innovative approaches to contraceptive delivery offer the most promise. 


\section{Lay summary}

Despite many contraceptive options being available in the United Kingdom, a high number of pregnancies are still unplanned, meaning that they occur before they were wanted, or when they were not wanted at all. Mothers with unplanned pregnancies present later for maternity care and are more likely to have a premature and a low birth-weight baby. Both mother and child are at greater risk of violence and abuse when the pregnancy is unplanned. Contraception in Scotland is currently available from GPs, sexual health clinics and pharmacies, but lots of women still don't use it, or don't use the most effective methods. The research in this study looks at different ways to offer contraception from those that are currently available.

In our first study we looked at increasing the places where women could receive contraception to include the community pharmacy, where currently just emergency contraceptive pills and condoms are available. Women who were already using the contraceptive injection could receive up to three injections from one of eleven trained community pharmacists, instead of from their doctor or at a clinic. This might make this method more attractive to women, and as this method is more effective than either condoms or contraceptive pills, the two most popular methods, this might reduce unplanned pregnancy rates. Unfortunately there were lots of problems with this study. Staff at the pharmacy sometimes gave women wrong information, and women were not able to attend the pharmacy when they wanted to, so actually a lot of women participating received their injection at the clinic after all. In questionnaires afterwards some women were enthusiastic about getting their injection at the pharmacy but some pointed out the problems that they had experienced. The pharmacists were interviewed and they were generally positive about the idea, but they did not always appreciate the difficulties the women experienced.

This thesis also investigated offering women contraceptive methods in the time just after they have had a baby. When this research was started, women in Scotland were rarely offered contraceptive options at this time. One contraceptive method that is very effective is intrauterine contraception, a contraceptive device that is fitted inside 
the womb. We offered all women who were having an elective (not an emergency) caesarean section the option of having intrauterine contraception inserted at their operation, after their baby was delivered. We then followed all these women up with a visit at six weeks, and a telephone call at three, six and twelve months. Very few devices came out, and a year later a very high number of women who we contacted were still using their device for contraception, and were very happy with it. Offering this effective contraceptive method at this time therefore seems to be convenient for women and straightforward.

We concluded that not all ways to expand contraceptive delivery work for women, but some do, and it is a good idea to conduct careful research to make sure new ideas work well before they are adopted. 


\section{Acknowledgements}

My research fellowship was made possible due to an unrestricted grant from HRA Pharma. I am very appreciative of their support, and of my supervisors' support in securing the funding.

I am incredibly grateful to all of the participants who so generously gave up their time to participate in research both contained in this thesis and conducted throughout the course of my fellowship. The generosity of research participants in contributing towards the advancement of medical knowledge is always humbling to me.

Thank you to the staff at Chalmers Centre, Edinburgh, St. John's Hospital, Livingston and the Simpson Centre for Reproductive Health in Edinburgh for your support, enthusiasm and flexibility which are so necessary when conducting medical research.

I am extremely grateful to both of my supervisors, Professors Sharon Cameron and Anna Glasier, for the fantastic opportunity to complete this $\mathrm{PhD}$, their constant encouragement of me, and for all of the unique and wonderful opportunities that completing this research fellowship has given me access to.

I would like to thank my amazing family, lovely friends and treasured quartet for their unending support and love throughout completion of this $\mathrm{PhD}$. A final and special thank you must go to my Dad, Professor Simon Heller, for assistance both practical and emotional, and for passing on his passionate belief in medical research as the best tool we have to improve patient care. 


\section{List of abbreviations used in this thesis}

$\begin{array}{ll}\text { COC } & \text { Combined oral contraceptives } \\ \text { Cu-IUD } & \text { Copper intrauterine device } \\ \text { DMPA-IM } & \text { Depot medroxyprogesterone acetate: intramuscular } \\ \text { DMPA-SC } & \text { Depot medroxyprogesterone acetate: subcutaneous } \\ \text { DNA } & \text { Did not attend } \\ \text { EHC } & \text { Emergency hormonal contraception } \\ \text { GP } & \text { General practitioner } \\ \text { HCP } & \text { Health care provider } \\ \text { IUC } & \text { Intrauterine contraception } \\ \text { IUD } & \text { Intrauterine device } \\ \text { IUS } & \text { Intrauterine system } \\ \text { LARC } & \text { Long acting reversible contraception } \\ \text { LNG } & \text { Levonorgestrel } \\ \text { LNG-IUS } & \text { Levonorgestrel intrauterine system } \\ \text { OCP } & \text { Oral contraceptive pill } \\ \text { OTC } & \text { Over the counter } \\ \text { PGD } & \text { Patient group direction }\end{array}$




\section{Table of Contents}

Chapter 1 Introduction.................................................................................... 1

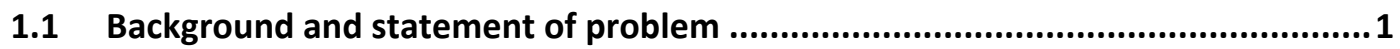

1.1.1 Unintended pregnancy in a national and global context ................................ 1

1.1.2 Contraception used in the United Kingdom .................................................... 4

1.1.3 Long acting reversible contraception ............................................................. 7

1.1.4 Contraceptive delivery: current service models........................................... 10

1.1.5 Contraceptive care: no missed opportunities .............................................. 12

1.1.6 Why expand contraceptive uptake? .......................................................... 13

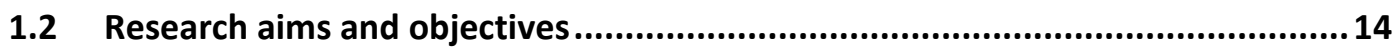

1.3 The importance of research contained in this thesis ......................................... 15

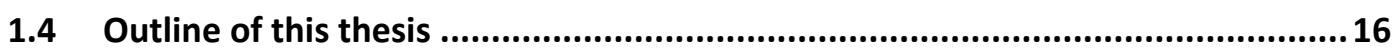

Chapter 2 Delivering sexual health at the community pharmacy: mixed methods literature review .................................................................................... 18

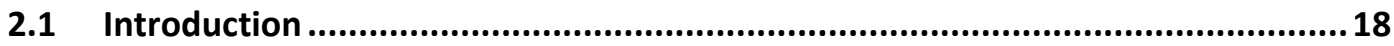

2.1.1 Justification for the methodology .............................................................. 19

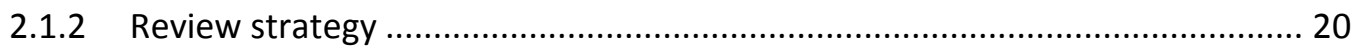

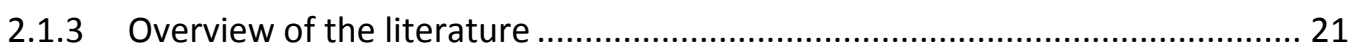

2.2 Pharmacists' thoughts about their role in dispensing contraception ..................25

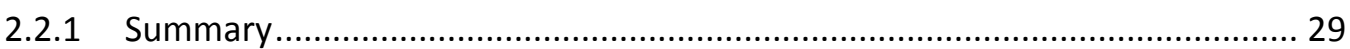

2.3 Patients' and communities' thoughts about pharmacists dispensing

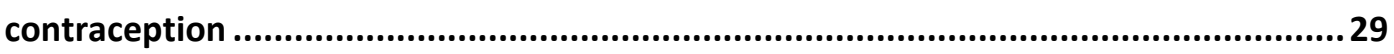

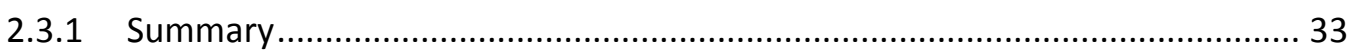

2.4 Emergency hormonal contraception in the pharmacy......................................33

2.4.1 Evidence for EHC in the pharmacy …………............................................. 34

2.4.2 'Mystery shopping' for EHC at pharmacies ...................................................... 36

2.4.3 Pharmacist and other healthcare professionals' attitudes to EHC.................. 37

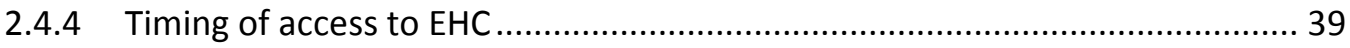

2.4.5 Ongoing contraception after EHC use ....................................................... 41

2.4.6 Patients' experience of EHC from pharmacies .............................................. 43

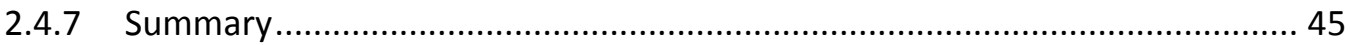


2.5 Ongoing contraception services delivered or facilitated by the pharmacy..........46

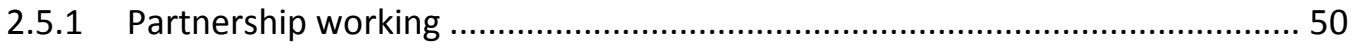

2.5 .2 Summary ................................................................................. 51

2.6 Long Acting Reversible Contraception (LARC) at the pharmacy ......................52

2.7 The need for further research...............................................................5 52

2.8 The literature review in the context of this thesis ..........................................53

Chapter 3 Background research for a pilot study examining contraceptive

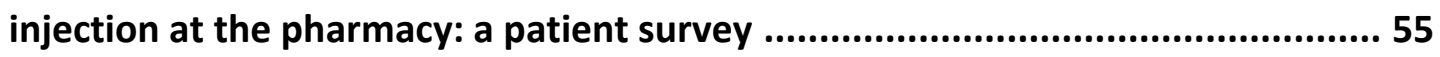

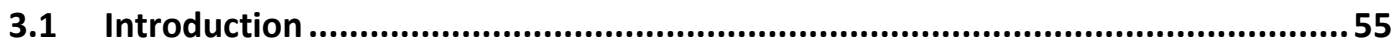

3.2 Justification for the methodology ............................................................ 55

3.3 Patients' preparedness to attend a pharmacy for contraceptive injections: a

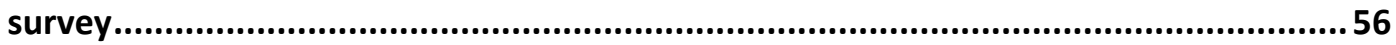

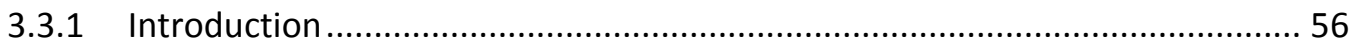

3.3.2 Methods........................................................................................ 58

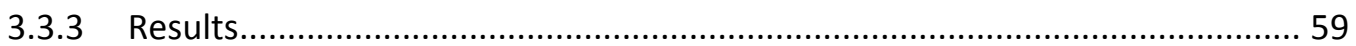

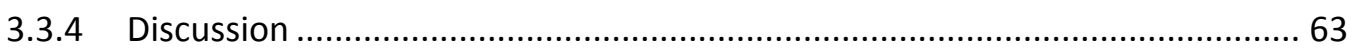

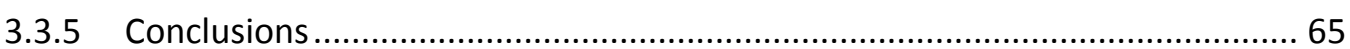

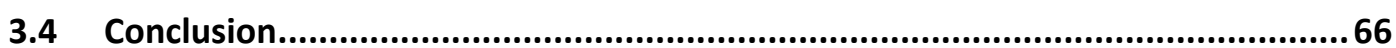

Chapter 4 Delivering injectable contraception at the community pharmacy:

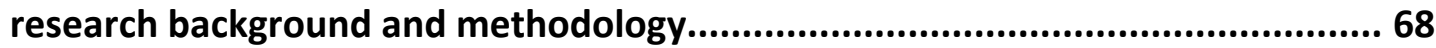

4.1 Justification for the research ......................................................................6 68

4.2 Background to the research hypothesis .................................................. 69

4.3 Designing the pilot study ....................................................................... 71

4.4 Identification and recruitment of pharmacies.............................................. 71

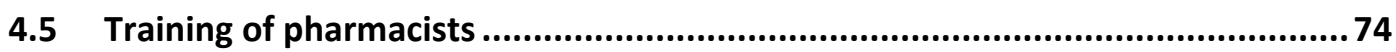

4.6 Patient population and recruitment ..................................................... 75

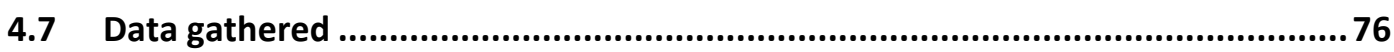

Chapter 5 Delivering injectable contraception at the community pharmacy:

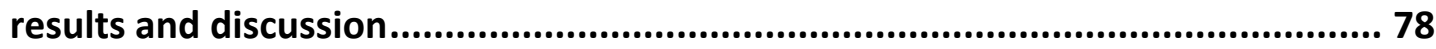

5.1 Introduction .............................................................................. 78

5.2 Results: demographics of participants ...................................................... 78 
5.3 Results: injections received at the pharmacy .................................................. 79

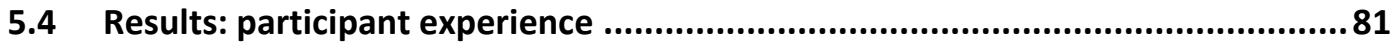

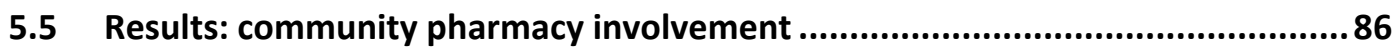

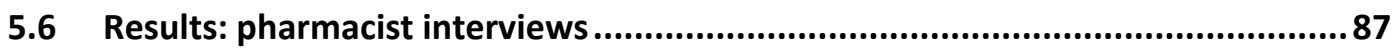

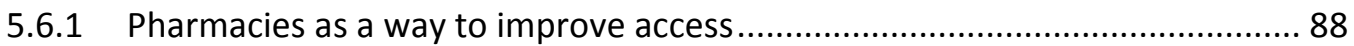

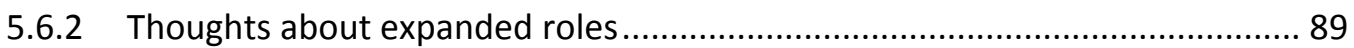

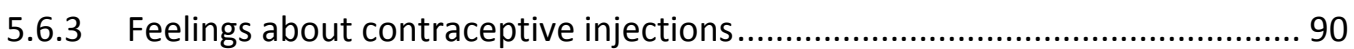

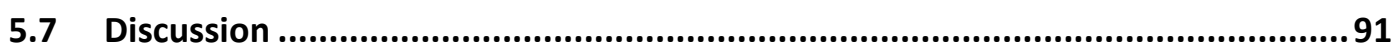

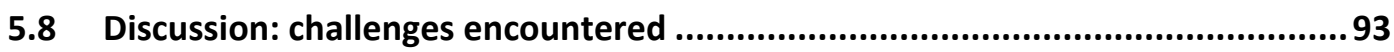

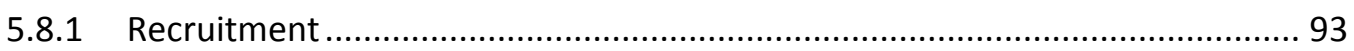

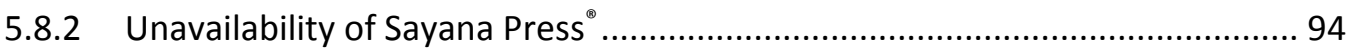

5.8.3 Licensing of Sayana Press ${ }^{\bullet}$ for self-injection.................................................. 95

5.8.4 Working with community pharmacies ......................................................... 95

5.9 Suggestions for future research .................................................................. 100

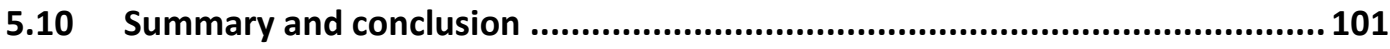

Chapter 6 Postpartum contraception: a missed opportunity to prevent unintended pregnancy and short inter-pregnancy intervals ...........................103

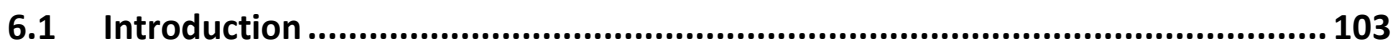

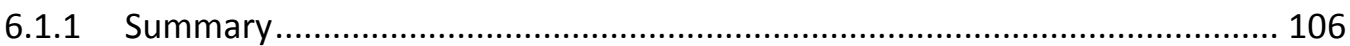

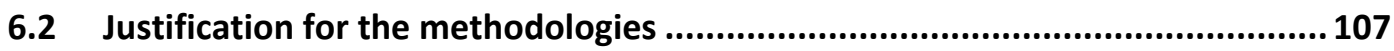

6.3 Postpartum contraception: a missed opportunity to prevent unintended pregnancy and short inter-pregnancy intervals .......................................................... 108

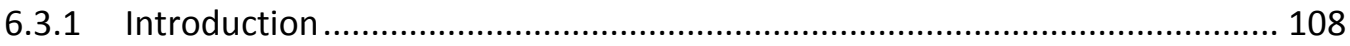

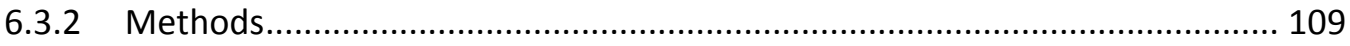

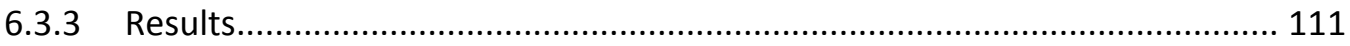

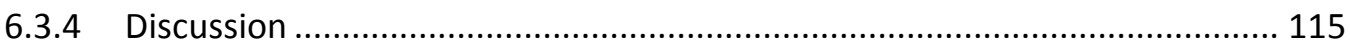

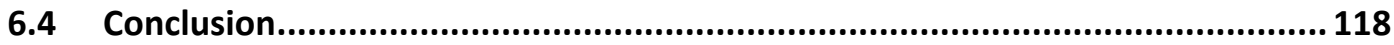

Chapter 7 Intrauterine contraception inserted at caesarean section..............119

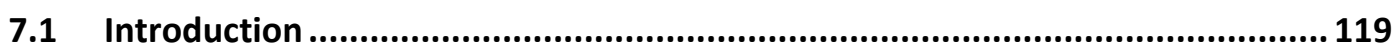

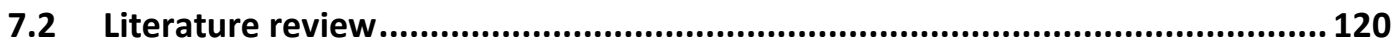

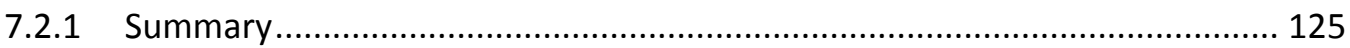

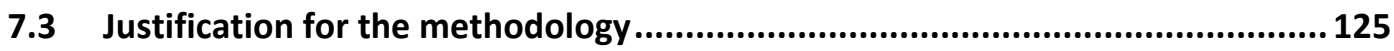




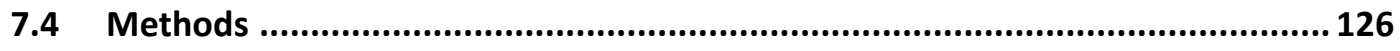

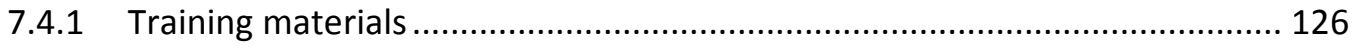

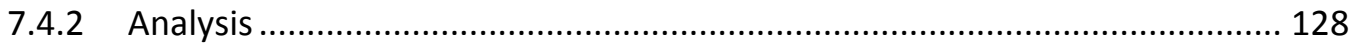

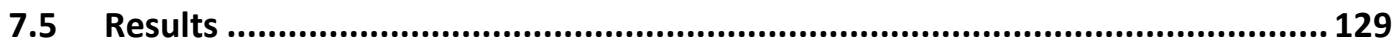

7.5.1 Outcomes and complications …………….............................................. 130

7.5.2 Continuation and satisfaction rates............................................................. 134

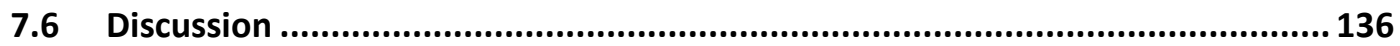

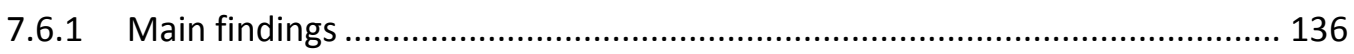

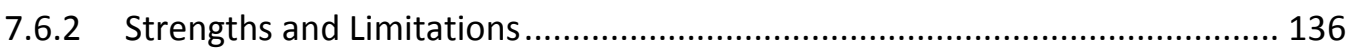

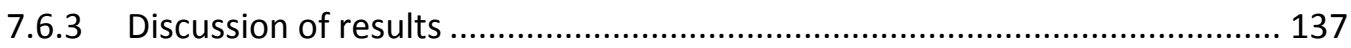

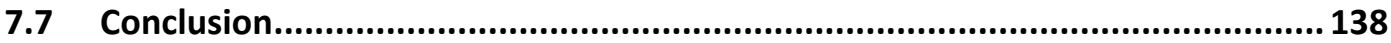

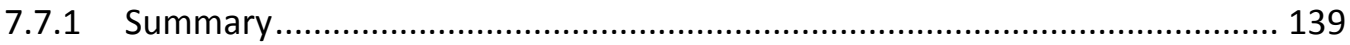

Chapter 8 Conclusions, Clinical Implications and Directions for Future Research 140

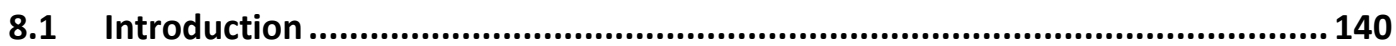

8.2 Expanding contraception uptake to prevent unplanned pregnancy ................ 140

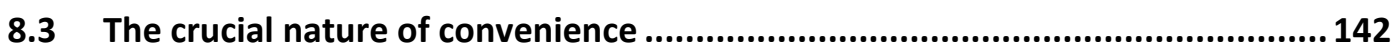

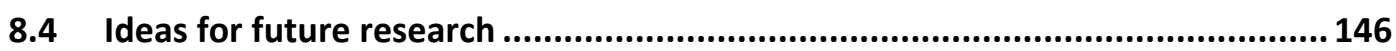

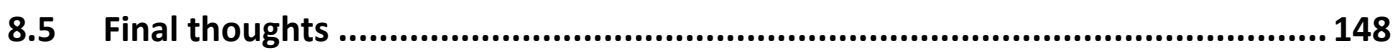

Appendix 1 Questionnaires administered in research described in this thesis

Appendix 2 Publications resulting from research described in this thesis appearing as published 


\section{Chapter 1 Introduction}

This chapter details the background and rationale for undertaking the research described in this thesis. I examine the problem of unplanned pregnancy in a global, national and Scottish context, and review the contraceptive options for women in use in the United Kingdom. Current national service for contraceptive provision is then outlined, and novel options for contraceptive access are identified. The aims, rationale and justification for the research are then presented.

\section{A note on the timing of the research explored in this introduction}

Contraception delivery in Scotland and the UK in the field of maternity and postpartum care has changed considerably since this thesis was commenced. International literature shows an increasing focus on starting the most effective methods immediately postpartum from 2011 (Celen et al., 2011; Bhutta et al., 2011; Glazer et al., 2011; Levi et al., 2012; Curry et al., 2012; Tocce et al., 2012b; Tocce et al., 2012a). However immediate postpartum contraception did not gain attention in the United Kingdom until 2015. This was due in part to research to which I contributed (Heller et al., 2016; Cameron et al., 2017; The Scottish Government, 2015), and guidelines produced at the beginning of 2017, which cite research described in this thesis (Faculty of Sexual and Reproductive Healthcare, 2017). This introduction will therefore concentrate on contraception services when the research commenced, in the summer of 2013. The concluding chapter of the thesis will reflect the changed landscape, and examine the most recent evidence for contraceptive delivery.

\subsection{Background and statement of problem}

\subsubsection{Unintended pregnancy in a national and global context}

Unintended pregnancy remains an ongoing problem in the United Kingdom, despite a variety of contraceptive options available to women. The idea of an unintended or unplanned pregnancy generally describes pregnancies that are either unwanted (occurring when no pregnancy was desired), or mistimed (occurring earlier than a desired pregnancy) (Santelli et al., 2003). However, the dichotomous concept of unintended versus intended pregnancy may be inadequate, as women's relationship 
to the 'wantedness' of their pregnancy may alter with pregnancy course and outcome (Trussell et al., 1999). This variability complicates answers to interviews and surveys about pregnancy intentions by suggesting that such research may only capture a snapshot of mutable feelings.

The London Measure of Unplanned Pregnancy was developed in response to demands for a reconceptualising of unplanned pregnancy. It used interviews over time with women who had a variety of pregnancy outcomes to build a valid measure of unplanned pregnancy. The measure was then rigorously tested for reliability, and enabled women to express mixed feelings about their pregnancy irrespective of outcome (Barrett et al., 2004). The most recent National Survey of Sexual Attitudes and Lifestyles (Natsal-3), one of the largest and most scientific studies of sexual behaviour, used the London Measure of Unplanned Pregnancy (LMUP) to examine pregnancy intentions of 591 women who had a pregnancy with a known outcome in the year preceding their interview. $16.2 \%$ of pregnancies were unplanned, $29 \%$ were classed as ambivalent, and 54.8\% were planned (Wellings et al., 2013). Similar data from Edinburgh, Scotland in 2006 showed that $65.6 \%$ of total pregnancies (ending in abortion, miscarriage or a continuing pregnancy) are intended, $25.8 \%$ were ambivalent and 8.6\% were unintended (Lakha and Glasier, 2006). Whether or not to classify ambivalent pregnancies as unintended is complex. The developers of the LMUP emphasise that the scale represents a spectrum rather than definitive categories (Barrett et al., 2004). Other surveys where women are offered only the option of intended pregnancy (the respondent wanted to have a baby at the time the pregnancy occurred) or unintended pregnancy have put an unplanned pregnancy rate as high as $49 \%$ (Finer and Henshaw, 2006), suggesting that when presented with only two choices women classify a larger proportion of pregnancies as unintended. Despite the inherent complexities as described above, the dichotomy of intended/unintended pregnancy has been essential to researchers and public health practitioners working to prevent unwanted childbearing, and will be used in this thesis. 
The impact of unintended pregnancy on society is substantial. Mothers with unintended pregnancies present later for antenatal care, are more likely to have a low birth weight infant and to deliver prematurely (Shah et al., 2011) and are less likely to breastfeed, or breastfeed for a shorter duration (Gipson et al., 2008). Evidence suggests a positive association between unintended pregnancy and child abuse, and between unintended pregnancy and child mortality (Mohllajee et al., 2007). One population-based study from the United Kingdom found that children registered with the child protection agency by the age of six were nearly three times likely to be a result of a pregnancy their mother considered unintended (Sidebotham et al., 2003). Unintended pregnancy is also likely to have a substantial impact on maternal wellbeing; unintended childbearing carries a significantly increased risk of maternal depression, anxiety and psychological ill health (Gipson et al., 2008). Women with unwanted or mistimed pregnancies are also more likely to be a victim of physical violence than women with intended pregnancies, regardless of other characteristics (Gazmararian et al., 1995). In addition, women with unintended pregnancies are far more likely to live in poverty, and to be made poorer by raising children or paying for abortions (Forrest, 1994).

Termination of pregnancy is also an important outcome of unplanned pregnancy. Although not all pregnancies ending in termination are unplanned, evidence suggests that only about $5 \%$ of pregnancies that result in a termination were initially an intended pregnancy (Finer and Henshaw, 2006), and figures from Edinburgh, Scotland put this figure at less than 1\% (Lakha and Glasier, 2006; Schünmann and Glasier, 2006). Unintended pregnancies therefore account for the vast majority of abortions. In Scotland there were 12,082 abortions performed in 2015. 98.3\% of these were carried out because "continuance of the pregnancy would involve risk, greater than if the pregnancy were terminated, of injury to the physical or mental health of the pregnancy woman" (NHS Scotland Information Services Division, 2016a). These abortions were carried out under 'Ground C' of the 1967 abortion act (1967). Abortions for fetal anomaly (and perhaps therefore more likely to have been originally a planned or wanted pregnancy) therefore accounted for less than $2 \%$ of abortions in Scotland. Although a termination of pregnancy in the United Kingdom is 
an extremely safe and straightforward procedure, whether medical or surgical (Kulier et al., 2001; Kulier et al., 2011; Say et al., 2002), any reduction in unplanned pregnancy would reduce the termination of pregnancy rate.

Abortion and its status in many countries makes the global burden of unplanned pregnancy extensive. Estimates are that each year in 2010-2014 there were 35 abortions per 1000 women aged 15-44 worldwide. Globally 25\% of pregnancies end in abortion. Extensive data from 2010-2014 were published in 2016, obtained from official statistics and published or unpublished national studies. Abortion legality does not affect abortion rates, this research showed a rate of 37 abortions per 1000 women in countries where abortion is prohibited completely or allowed only to save a woman's life, and 34 where it is available on request (Sedgh et al., 2016). In countries where abortion is illegal and therefore unsafe, unintended pregnancy is a major contributor to maternal morbidity and mortality (Santelli et al., 2003). A WHO systematic analysis estimated that abortions were responsible for $7.9 \%$ of maternal deaths between 2003-2009, equivalent to approximately 193000 deaths. Furthermore the authors identify that this is likely an underestimation due to illegality and religious and cultural stigma associated with abortion in some countries (Say et al., 2014).

\subsubsection{Contraception used in the United Kingdom}

Women in the United Kingdom have a wide variety of contraceptive options available to them, but limited data on current use are available. Evidence from 2009 suggests that one in four women under 50 are not using any method of contraception, of whom just under half in a sexual relationship with an opposite sex partner (Lader, 2009). Table 1-1 demonstrates all methods in use by women aged 16-49. This data is based on the National Statistics Opinion Survey during 2008/09, and draws on answers from just 1093 women. These figures may well have changed but this survey is no longer in existence and there are no more up to date data. 
Table 1-1 Contraception use in the United Kingdom 2008/9, adapted from Lader 2009

\begin{tabular}{|c|c|}
\hline Current use of contraception & Percentage who used \\
\hline \multicolumn{2}{|l|}{ Non-surgical } \\
\hline Pill & 25 \\
\hline Minipill & 6 \\
\hline Combined pill & 16 \\
\hline Male condom & 25 \\
\hline Withdrawal & 4 \\
\hline IUD & 6 \\
\hline Injection & 3 \\
\hline Implant & 1 \\
\hline Patch & 0 \\
\hline Safe period/rhythm method/Persona & 2 \\
\hline Cap/diaphragm & 0 \\
\hline Foams/gels & 1 \\
\hline Hormonal IUS & 2 \\
\hline Female condom & 1 \\
\hline Emergency contraception & 1 \\
\hline Total at least one method non-surgical & 58 \\
\hline \multicolumn{2}{|l|}{ Surgical } \\
\hline Sterilised & 6 \\
\hline Partner sterilised & 11 \\
\hline Total at least one method & 75 \\
\hline \multicolumn{2}{|l|}{ Not using a method } \\
\hline No heterosexual relationship & 13 \\
\hline Sterile after another operation & 2 \\
\hline Wants to get pregnant & 2 \\
\hline Pregnant now & 2 \\
\hline Going without sex to avoid pregnancy & 1 \\
\hline Unlikely to conceive because of menopause & 1 \\
\hline Possibly infertile & 1 \\
\hline Doesn't like contraception & 1 \\
\hline Other reason & 3 \\
\hline Total not using a method & 25 \\
\hline
\end{tabular}

The contraceptive pill and male condom are by far the most popular methods of contraception, accounting for $50 \%$ of contraceptive use between them. However, with this relatively small sample size it may be difficult to capture data on methods used by fewer women such as the diaphragm, contraceptive patch, and contraceptive 
implant. In addition the contraceptive implant in particular has seen steady growth in popularity since 2008 when this data was collected (Cea Soriano et al., 2014b) (see figure 1-1).

Figure 1-1 Annual incidence and prevalence of long-acting reversible contraceptive use from 2004 to 2010, reproduced with permission from Cea Soriano et al 2014b

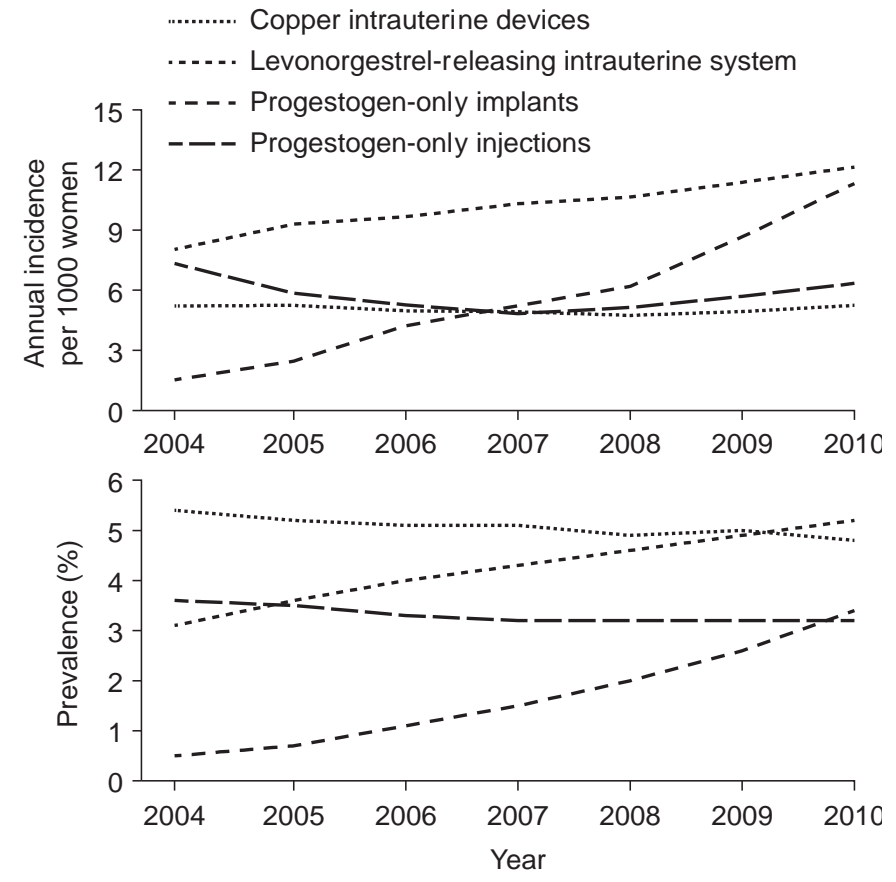

Data from 2013 examining methods prescribed by general practitioners also suggests that combined oral contraception remains by far the most popular prescribed method of contraception in the primary care setting. Anonymised patient records of women aged 12-49 in the year 2008 from a medical research database were analysed. 574 185 women were eligible, of whom 194054 (34\%) had used one of the included contraceptive methods during the study. These were the combined oral contraceptive pill, progestogen-only pill, copper IUD, levonorgestrel-releasing intrauterine system, progestogen-only implant, contraceptive injection and contraceptive patch. The study underlines the results from the National Statistics Opinion Survey, finding that oral contraceptives are used by $21.8 \%$ of women aged 12-49 (Cea Soriano et al., 2014a). The breakdown of the methods used by participants in the study is depicted in figure $1-2$. 
Figure 1-2 Prevalence of use of contraceptive methods in 2008 among women aged 12-49 (reproduced from Cea Soriano et al 2014a)

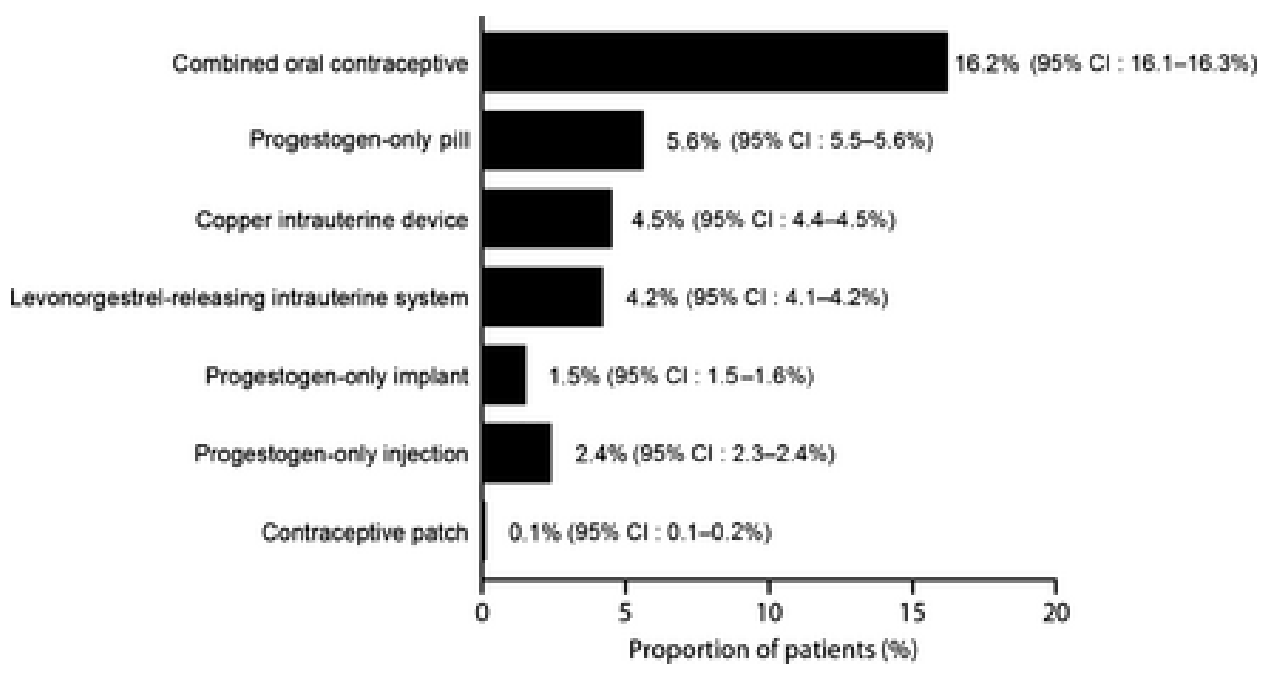

\subsubsection{Long acting reversible contraception}

Contraceptive failure can be examined using either 'perfect' use, or 'typical' use: use of the method by an average person in a real life setting, including inconsistencies and imperfect use. Table 1-2 demonstrates the typical and perfect use failure rates of available contraceptive methods used in the United Kingdom.

Table 1-2 Percentage of women in the United States experiencing an unintended pregnancy during the first year of use. Adapted from Trussell 2011

\begin{tabular}{|l|l|l|}
\hline Method used & Typical use & Perfect use \\
\hline No method & 85 & 85 \\
\hline Fertility awareness based methods & 24 & $0.4-5^{*}$ \\
\hline Withdrawal & 22 & 4 \\
\hline Female condom & 21 & 5 \\
\hline Male condom & 18 & 2 \\
\hline Diaphragm & 12 & 6 \\
\hline Combined pill and progestin-only pill & 9 & 0.3 \\
\hline Contraceptive patch & 9 & 0.3 \\
\hline Contraceptive vaginal ring & 9 & 0.3 \\
\hline Contraceptive injection (Depo-Provera) & 6 & 0.2 \\
\hline Copper intrauterine device & 0.8 & 0.6 \\
\hline Levonorgestrel intrauterine system & 0.2 & 0.2 \\
\hline Contraceptive implant (Implanon) & 0.05 & 0.05 \\
\hline Female sterilisation & 0.5 & 0.5 \\
\hline
\end{tabular}




\begin{tabular}{|l|l|l|}
\hline Male sterilisation & 0.15 & 0.10 \\
\hline *Depending on method used & \\
\hline \multicolumn{2}{|l|}{ Methods shaded in blue are long acting reversible contraception methods } \\
\hline
\end{tabular}

The LARC (long acting reversible contraceptive) methods (shaded in blue) are demonstrably the most effective reversible methods to avoid unplanned pregnancy, mainly due to the low discrepancy between their typical and perfect use. A large prospective cohort study in St. Louis, USA examining the relative effectiveness of LARC found that participants using contraceptive pills, patches and vaginal rings, had a risk of contraceptive failure 20 times higher than those using LARC (Winner et al., 2012). The contraceptive injection, or Depo-Provera ${ }^{\circ}$, can be classed as a LARC method, but as it requires user input every 12-13 weeks it is not usually included when data is collected specifically about LARC (NHS Scotland Information Services Division, 2016b). The LARC methods therefore consist of the contraceptive implant, a subdermal rod that releases progestogen, the copper IUD (intrauterine device) and the levonorgestrel-releasing intrauterine device, also known as an intrauterine system, or IUS. Because LARC methods are the most effective at preventing unplanned pregnancy, national strategies for sexual health include goals to improve the uptake of LARC. In Scotland the 2015 update to the Sexual Health and Blood Borne Virus Framework noted the increase in LARC since the framework's inception in 2011, and called for further increases (The Scottish Government, 2015).

Recent data from Scotland suggests that the LARC prescribing rate for women of reproductive age is 52.1 per 1000 women (NHS Scotland Information Services Division, 2016b). Figure 1-3 demonstrates the use of LARC by age group. Whilst it is difficult to compare different methods of investigating contraceptive use, data from the national opinion survey suggests that up to $59 \%$ of women of reproductive age require effective contraception (Lader, 2009). It therefore makes sense, from a public health point of view, for a much greater proportion of these women to be using LARC methods. Evidence suggests that prevalence of LARC methods is increasing UK-wide: between 2004 and 2010 use of the progestogen-only implant 
rose from 1.5 to 11.3 per 1000 women, and use of the LNG-IUS from 3.1 in 2004 to 5.2 in 2010 (Cea Soriano et al., 2014b).

However, despite their efficacy at preventing the main outcome contraceptive users wish to avoid, prevalence remains low. There are many reasons why patients choose not to use LARC methods, despite their demonstrably greater efficacy at preventing unplanned pregnancy. Evidence from Scotland in 2008 shows that pills and condoms are more familiar to women, who may hold incorrect beliefs about LARC, and suggests women may not appreciate the differences in efficacy with typical use (Glasier et al., 2008). GPs (general practitioners) may be contributing to the low LARC uptake, as evidence from 2011 showed that $92 \%$ of GPs questioned $(n=71)$ identified the contraceptive pill as their first-line method of contraception for a young nulliparous woman, despite $85 \%$ of respondents recognising that LARC were the most effective methods (Middleton et al., 2011).

Figure 1-3 LARC methods prescribing rate per 1000 women by age group 2015/16. Reproduced from NHS Scotland Information Services Division $2016 \mathrm{~b}$

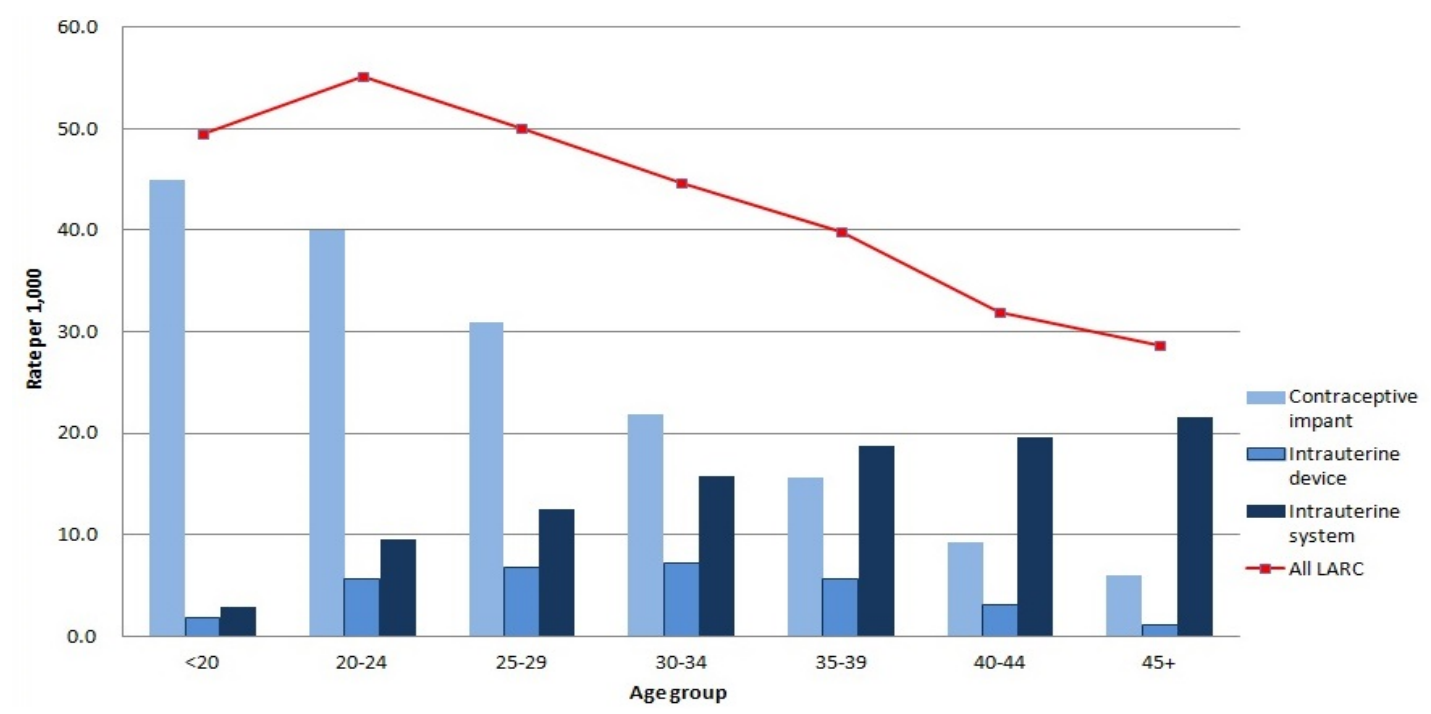

A recent article argued that there has been an unnecessary prioritisation of LARC methods over short-acting methods, using a computer simulation model to critique Winner et al, arguing that much of LARC's demonstrated efficacy in reducing unplanned pregnancy rates is due to a reduction in non-users and condom users, and that commencing all non-users on short-acting methods would have almost as 
significant an effect on unplanned pregnancy (Karpilow and Thomas, 2017).

However, there is overwhelming evidence that non-users do not use contraception for a multiplicity of reasons, notably thinking they will not get pregnant (Jones et al., 2002; Moreau et al., 2005), believing that their partner wants them to be pregnant (Stevens-Simon et al., 2005), their partner being in control of whether or not they use contraception (Williams et al., 2008), feeling that pregnancy should not or cannot be prevented (Borrero et al., 2015) and numerous other individual, interpersonal and societal factors (Ayoola et al., 2007). It is therefore not as simple as a computer simulation might suggest, and moving both non-users and users of short-acting methods to LARC has the potential to significantly impact unplanned pregnancy rates.

\subsubsection{Contraceptive delivery: current service models}

In the United Kingdom all available contraception is free of charge for users. The NHS website states that patients can receive contraception from most GP surgeries, community contraception clinics, some genitourinary medicine clinics, sexual health clinics and some young people's services (NHS Choices Website, 2015). There are no fixed rules about which of these services should deliver contraception, and as a result availability is not guaranteed, or necessarily easy to predict. In Scotland, the Primary Medical Services (Scotland) Act 2004 outlined the terms by which health services are contracted by the local NHS Board from GPs. The essential services that must be delivered under this contract are the management of patients who are ill or who believe themselves to be ill, the management of chronic disease, the management of patients who are terminally ill, and primary care services in core hours for accidents and emergencies. Contraceptive services are not deemed to be an essential service, and are instead deemed an additional service, which any practice can opt out of providing if they wish (2004). Nevertheless, evidence from the United Kingdom (there is no specific Scottish data available) suggests that GPs are the most common provider of contraceptive supplies.

Data from the National Survey of Sexual Attitudes and Lifestyles in 2010-2012 demonstrates that the source of contraceptive supplies used by the largest proportion 
of women were GPs, with $59.1 \%$ of sexually active women reporting use of GP services in the last year. This is almost identical to the proportion obtaining contraception from GP services in 1999-2001 (French et al., 2009). In 2010-12, 23\% of women interviewed obtained supplies from community services, largely family planning clinics (14.4\%). 8.5\% obtained supplies from genito-urinary medicine clinics, reflecting an increasing move towards integration between these two specialist services, at least in some parts of the country (French et al., 2017). Figure 1-4 demonstrates service use for contraceptive supplies and/or advice in the last year for women, based on answers to Natsal-3.

Figure 1-4 Sources used to obtain contraception in the past year among women aged 16-44 who report vaginal sex in the past year and obtaining contraceptives. Reproduced from French et al 2017

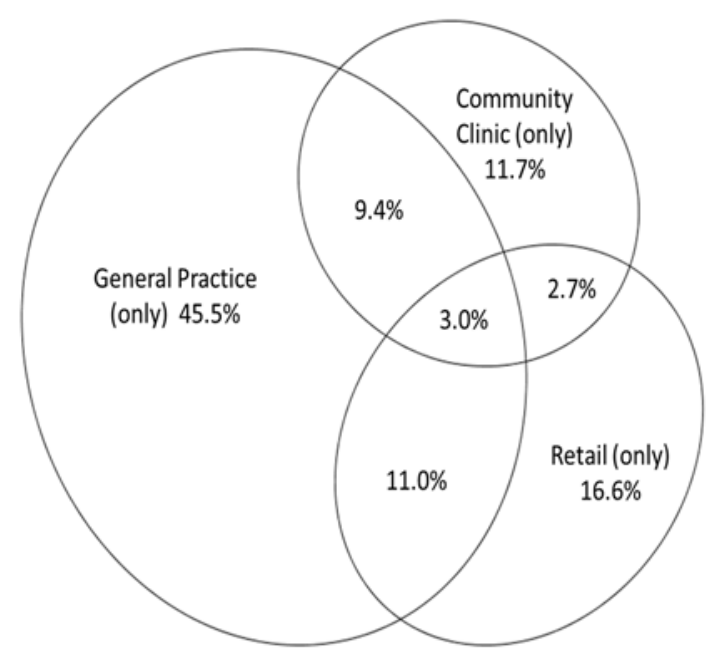

In Scotland specialist sexual health services are the main providers of contraception after GPs (NHS Scotland Information Services Division, 2016b), though contraceptives may sometimes be given in a hospital setting. This is particularly true after termination of pregnancy (Cameron et al., 2012a), and contraceptive provision after abortion was a key priority of the Scottish Sexual Health and Blood Borne Virus Strategy, both originally and in the 2015 update (The Scottish Government, 2011; The Scottish Government, 2015). Currently contraception is also provided very occasionally in the maternity setting (Cameron et al., 2017; Smith and McLellan, 2014). Emergency hormonal contraception (EHC) is also delivered 
through all routes mentioned above, though the vast majority of EHC is delivered through pharmacies based in the community (Marston et al., 2005). In Scotland EHC is available free of charge in the pharmacy as well as the clinical setting (Cameron et al., 2012c).

\subsubsection{A note on delivery of contraceptive care in England and Wales}

Delivery of contraceptive services in England and Wales may be more fragmented due to the recent introduction of clinical commissioning groups, and commissioning of sexual healthcare and contraceptive services by various community, education, pharmacy or private sectors (National Institute for Health and Care Excellence (NICE), 2016). The Royal College of General Practitioners has raised concerns about the implications of clinical commissioning of sexual and reproductive healthcare in England, particularly with regards to ensuring LARC provision and holistic care for patients (Royal College of General Practitioners, 2014). A discussion of this is outwith the scope and relevance of this research.

\subsubsection{Contraceptive care: no missed opportunities}

Contraception is a unique healthcare intervention, in that it is delivered to a well population who may not consider themselves in need of it. Evidence from the United States shows that almost one in five women aged 18-29 believe themselves very likely to be infertile (Polis and Zabin, 2012), and that $41 \%$ of women with an unintended pregnancy had thought either that they couldn't get pregnant at the time of the intercourse or that they or their partner were infertile (Nettleman et al., 2007). The perceived lack of need among the population combined with the unplanned pregnancy rate means that it needs to be as easy as possible for women to access contraceptive care with as few barriers as possible. The idea of contraception delivered opportunistically has been gradually adopted into policy in the area of termination of pregnancy. With a growing body of evidence that offering women contraception at the time of termination reduces unplanned pregnancy rates (Goodman et al., 2008; Bednarek et al., 2011; Cameron et al., 2012a), enthusiasm for the intervention has been reflected in policy and clinical guidance (The Scottish Government, 2011; The Scottish Government, 2015; Royal College of Obstetricians 
and Gynaecologists, 2011). However, when this research was commenced in 2013, the idea of offering contraception opportunistically at other healthcare interventions, notably after pregnancy ending in a live birth, was just appearing in the international literature (Celen et al., 2011; Bhutta et al., 2011; Glazer et al., 2011; Levi et al., 2012; Curry et al., 2012; Tocce et al., 2012a), but was not yet being discussed in the UK.

Figure 1-3 shows the variety of sources women use to access contraception. $16.6 \%$ of women only obtained contraception from retail settings (French et al., 2017). Currently the only contraception methods available in the retail setting are condoms and emergency hormonal contraception (EHC) (Department of Health, 2005). French et al note an increase in the use of retail outlets since similar data was gained in 1999-2001, and speculate that this increase may reflect the increase in use of EHC from pharmacies, suggesting that pharmacies therefore account for a significant source of contraception obtained at retail outlets (French et al., 2009). A possible expansion of contraception options could be to offer more contraceptive options in a pharmacy setting.

\subsubsection{Why expand contraceptive uptake?}

Unplanned pregnancy can be conceptualised as having three similar but separate antecedents. First, true contraceptive failure, the method used perfectly but pregnancy still occurring at the time of use. Secondly, a method used, but used inconsistently or imperfectly. Thirdly, no method used at the time of pregnancy occurring. The vast majority of unplanned pregnancies occur due to the second two (Jones et al., 2002; Larsson et al., 2002; Rasch, 2002; Schünmann and Glasier, 2006). The user independence of the LARC methods is the primary reason why they are more effective at preventing unplanned pregnancy, as it is largely impossible to use them inconsistently or imperfectly once they are inserted. A reduction in unplanned pregnancy therefore could take two approaches: moving women using less effective methods to more effective methods, and moving women using no contraception to using any method - ideally the most effective methods. 
Expanding contraceptive access to include the pharmacy would be likely to primarily achieve the second intervention, although some users might move from condoms to more effective methods, if they were more widely available. Documented systematic and medical barriers to women obtaining contraception include delayed initiation, clinician limitations and prescription requirements (Leeman, 2007). Removing some or all of these through pharmacy availability of some or all contraception options might increase uptake of the available methods.

The early focus on opportunistic delivery of contraception at the time of childbirth examines LARC methods, mirroring evidence from method initiation at the time of abortion (Goodman et al., 2008; Bednarek et al., 2011; Cameron et al., 2012a). The advantage of offering contraceptive at this time is twofold: evidence shows that this is a population who may not have access to accurate information about how to use contraceptives postpartum, and therefore may not use them at all (Murphy and British Pregnancy Advisory Service, 2014). In addition postpartum women are interacting with trained health professionals and therefore there is an opportunity to provide them with LARC methods. Opportunistic intervention could therefore enable women who are non-users to become users of the most effective methods.

Both offering the opportunity for non-users to become users through increased access, and increasing the uptake of the most effective methods which are far less likely to be used incorrectly and infrequently should act to reduce the unplanned pregnancy rate with a significant effect on both society and women's lives.

\subsection{Research aims and objectives}

In summary, unplanned pregnancy remains a significant problem in the United Kingdom and in Scotland, despite a wide range of effective contraceptive options available. The effect of these unplanned pregnancies on women and society is significant and wide reaching. The models of delivering contraceptive care differ from country to country, and within NHS boards in Scotland, but women access contraception from GPs, specialist sexual health clinics and retail outlets. Although there is clear evidence for opportunistic contraceptive delivery in the field of 
termination of pregnancy care that has been reflected in policy, there is limited evidence of similar enthusiasm for opportunistic contraceptive delivery elsewhere.

This aim of this research is to examine the expansion of contraceptive delivery beyond methods that were available to women at the time the research commenced, with the intent of providing evidence for new effective times and places of delivery.

The specific research objectives were as follows:

To explore a retail setting that currently delivers limited contraceptive options, namely the community pharmacy, to see whether there is scope for further provision of contraception in this location.

To explore the delivery of contraception in the postpartum setting, examining the need and evidence for this in a Scottish context. Specifically, is it possible for contraception to be routinely offered in the maternity setting as it currently is at termination of pregnancy?

\subsection{The importance of research contained in this thesis}

This research is important for a number of reasons.

- Increasing the sexual healthcare, including contraceptive care, delivered by community pharmacies has been called for at a national governmental level (Department of Health, 2004; Department of Health, 2005). It is a reasonable suggestion that, as patients already obtain some contraceptives at the pharmacy (French et al., 2009; French et al., 2017), increasing the contraceptives available here could increase the contraceptive uptake. This research therefore explores the evidence available for this proposition, and examines this possibility in a UK setting.

- There is good evidence that providing contraception, particularly the most effective methods, at the time of termination of pregnancy results in fewer unplanned pregnancies. This evidence is reflected in clinical guidance about contraceptive provision at the time of termination (Royal College of 
Obstetricians and Gynaecologists, 2011). This research aims to investigate the possibility of similar contraceptive provision in the maternity setting, through an examination of service provision, an assessment of patient opinion, and an observational study.

- Each of the possibilities outlined above describes contraceptive care that is currently beyond what is offered to patients. Examining evidence for these and investigating the ideas with appropriate research methodologies should provide general evidence about the possibilities for increasing future contraceptive delivery by looking outwith available service models.

\subsection{Outline of this thesis}

Chapter one provides an introduction to the subject, outlining the problem of unintended pregnancy in an global and national context, and introducing the current national contraception delivery systems designed to meet this need. It introduces the idea of expanding contraceptive delivery beyond current service models and suggests some options that will be further explored, namely contraceptive delivery from a pharmacy setting, and contraceptive delivery in the postpartum setting.

Chapter two provides a literature review of one possible contraceptive expansion: delivery of contraceptive care within the community pharmacy setting. The review examines available literature about service acceptability, both from a pharmacist and public point of view, and explores available evidence for service delivery from the pharmacy. It goes on to identify a possible area of exploration in a Scottish context, namely delivery of the contraceptive injection in the community pharmacy.

Chapter three presents published research undertaken in an urban sexual health centre to examine the demand for delivery of the contraceptive injection in a community pharmacy. A questionnaire was conducted with a potential recruitment population in order to assess feasibility of recruitment and service demand. This is outlined and results are presented.

Chapter four outlines the methodology for a pilot project that was conducted, offering participants contraceptive injections at the pharmacy. It outlines the 
strategies for identification of participating pharmacists, recruiting participants and designing how the pilot was conducted in practice.

Chapter five reports the results of this pilot. Both quantitative and qualitative results are presented. The results and implications of this research are then discussed.

Chapter six sets the scene for the delivery of contraception in the postpartum setting. The current national context is examined. There follows a published paper outlining research conducted on this subject, both identifying the need for postpartum contraception, and asking postpartum patients their thoughts on being offered contraception at this time.

Chapter seven focuses on a specific method of delivering postpartum contraception: insertion of intrauterine contraception at the time of caesarean section. A comprehensive literature review examines the evidence for this practice. An observational study that was conducted examining this option being made routinely available is then presented and discussed.

Chapter eight summarises the findings from the research presented in preceding chapters, and compares the findings to the research objectives set out in chapter one. It sets out the changes in clinical practice that have occurred locally and nationally as a result of this research. Finally it provides recommendations for future research and practice. 


\section{Chapter 2 Delivering sexual health at the community pharmacy: mixed methods literature review}

\subsection{Introduction}

In Chapter 1 I outlined the current unplanned pregnancy rate in Scotland, and argued that, in order to reduce as many barriers to contraception as possible for women, service delivery models can be expanded beyond those currently available. In this chapter, I will focus on the community pharmacist as a professional who has not traditionally delivered contraception, but whose potential to do so is gaining increasing attention. The term 'community pharmacist' will be used to denote a trained pharmacist who works in a retail community setting in a pharmacy, either independent or part of a chain. Community pharmacists are located within patients' communities, particularly in communities of deprivation, or rural settings (Todd et al., 2015). The involvement of community pharmacists in the sexual and reproductive healthcare of women could remove barriers of time, location and access, making it more possible for women to receive this intervention.

In 2004 a Department of Health White Paper entitled 'Choosing Health: making healthy choices easier' laid out a number of goals for improving the health of the nation. Within the chapter 'a health promoting NHS' special emphasis was put on the contribution to public health that pharmacists could make. "Working at the heart of the communities they serve, they have real opportunities to offer health messages and advice" (Department of Health, 2004). Sexual health was highlighted as a key component of this strategy. This idea was developed further in a 2005 White Paper, 'Choosing Health Through Pharmacy: a programme for pharmaceutical public health 2005-2015'. The paper noted the importance of an evidence base for pharmaceutical public health, perhaps because the 2004 White Paper was criticised for lacking one (Raine et al., 2004). The 2005 paper highlights the plan to involve pharmacies in sexual health, the pharmacist's role being defined as: offering emergency contraception under PGDs (Patient Group Directions - a legal framework allowing specified HCPs to supply and/or administer a medicine to a pre-defined group of 
patients, without the patient having to see a doctor or have medication prescribed (National Institute for Health and Care Excellence (NICE), 2015)), supplying condoms, signposting to other sources of advice and support, and offering sexual health advice and screening as part of an integrated system (Department of Health, 2001). Doctors working in sexual health have also noted the potential of the community pharmacist's role in sexual healthcare (Quilliam, 2013; Rani, 2008; Taylor, 2008).

\subsubsection{Justification for the methodology}

Although literature shows increasing enthusiasm for sexual healthcare delivered through community pharmacies (Chin, 2011; Rafie et al., 2014; Maderas et al., 2008; Eades et al., 2011), a systematic review of the contribution of pharmacists to public health concluded that there was insufficient evidence for their role in sexual health (Brown et al., 2012). There has been only one short review of the contribution of community pharmacy to sexual health (Chin, 2011). There have been reviews of the contribution to public health generally (Brown et al., 2012; Anderson et al., 2009; Anderson et al., 2005), and to the delivery of emergency hormonal contraception specifically (Anderson and Blenkinsopp, 2006). There has been one recent systematic review of pharmacy provision of reproductive health commodities to young people under 25 (Gonsalves and Hindin, 2016), and one review of the pharmacy's contribution to preventing unintended pregnancy, that focuses on United States data only (Farris et al., 2010). I planned to conduct research offering patients contraceptive care from their community pharmacy, and so it was crucial to assess the up to date evidence base surrounding this idea. This review will help to judge the potential of conducting contraception research within a community pharmacy setting, identify any gaps in the literature, and outline lessons learnt from research conducted in this field.

This chapter therefore provides a comprehensive review of the literature of contraceptive services delivered by community pharmacists. The review strategy is described. The findings of the literature review are then detailed, looking first at pharmacists' feelings on delivering sexual healthcare, then at patients' and the 
population's opinions, and finally an assessment of this as a strategy for delivering healthcare. The final section outlines gaps in the literature and provides the reasoning for my research that constitutes chapters 3,4 and 5 of this $\mathrm{PhD}$.

\subsubsection{Review strategy}

Literature searches were conducted in Medline, EMBASE and PubMed. Papers were limited from 2001 onwards as this is the year that emergency hormonal contraception became available from pharmacists over the counter in the United Kingdom. This service marked a change in the relationship of pharmacists to sexual health, and literature from after that point is more relevant to the current landscape. Searches were adapted to suit the different formats of the individual databases.

Search terms were: pharmacy OR community pharmacy OR community pharmacy services AND sexual health OR reproductive health OR contraception (exploded: contraception, barrier or contraception, postcoital or contraception or contraception, immunologic or contraception behaviour) OR birth control OR family planning OR family planning services. The search was limited to humans.

No formal protocol for the review was followed, as this was not a systematic review. Rather than answering a specific research question as a systematic review aims to do, it was more important to encompass the breadth of literature available, including position papers and literature looking at both patients' and pharmacists' perceptions and knowledge, as well as any assessment of delivery of sexual healthcare by the pharmacy that has taken place. Both qualitative and quantitative literature was examined, as each presents useful information for researchers on different aspects of the patient and pharmacist experience. Meta-analysis was not possible, and instead thematic analysis was used to draw out relevant themes. Papers were grouped together under these titles.

Literature searching provided me with 1,159 titles. These were screened for relevance by title and abstract (where title was inconclusive) to obtain 130 . I also screened references from four relevant reviews: two about public health in the pharmacy (Eades et al., 2011; Anderson et al., 2009), one about pharmacy provision 
of sexual health to young people (Gonsalves and Hindin, 2016), and one scoping review of the public response to pharmacy prescribing (Famiyeh and McCarthy, 2017). Examining the references of these reviews generated a further 23 papers. All 153 papers were all read in full, and 10 were rejected as not meeting the inclusion criteria. Only papers in English were included. Research articles, non-research articles (e.g. editorials) and posters/presentations from meetings were included if relevant. Papers met the inclusion criteria if they examined the idea of any type of contraceptive service, including condoms and EHC (emergency hormonal contraception) being delivered by a pharmacy, whether looking at the idea in theory or evaluating a current service, from the point of view of pharmacists, patients, or other healthcare professionals. Articles or research only about pharmacist knowledge about contraception, with no attitudinal component, were not included, as they were not felt to be a useful proxy for pharmacists' ability to deliver a service.

\subsubsection{Overview of the literature}

The availability of contraception from community pharmacies displays considerable geographic variation (Grindlay et al., 2013). Most of the literature available is from the United States, and from resource rich countries. A map showing the availability of oral contraceptives over the counter from the pharmacy is shown in figure 2-1 and detailed in table 2-1. Both demonstrate the considerable global variability. 
Figure 2-1 World map of contraception availability (reproduced from Grindlay et al 2013)

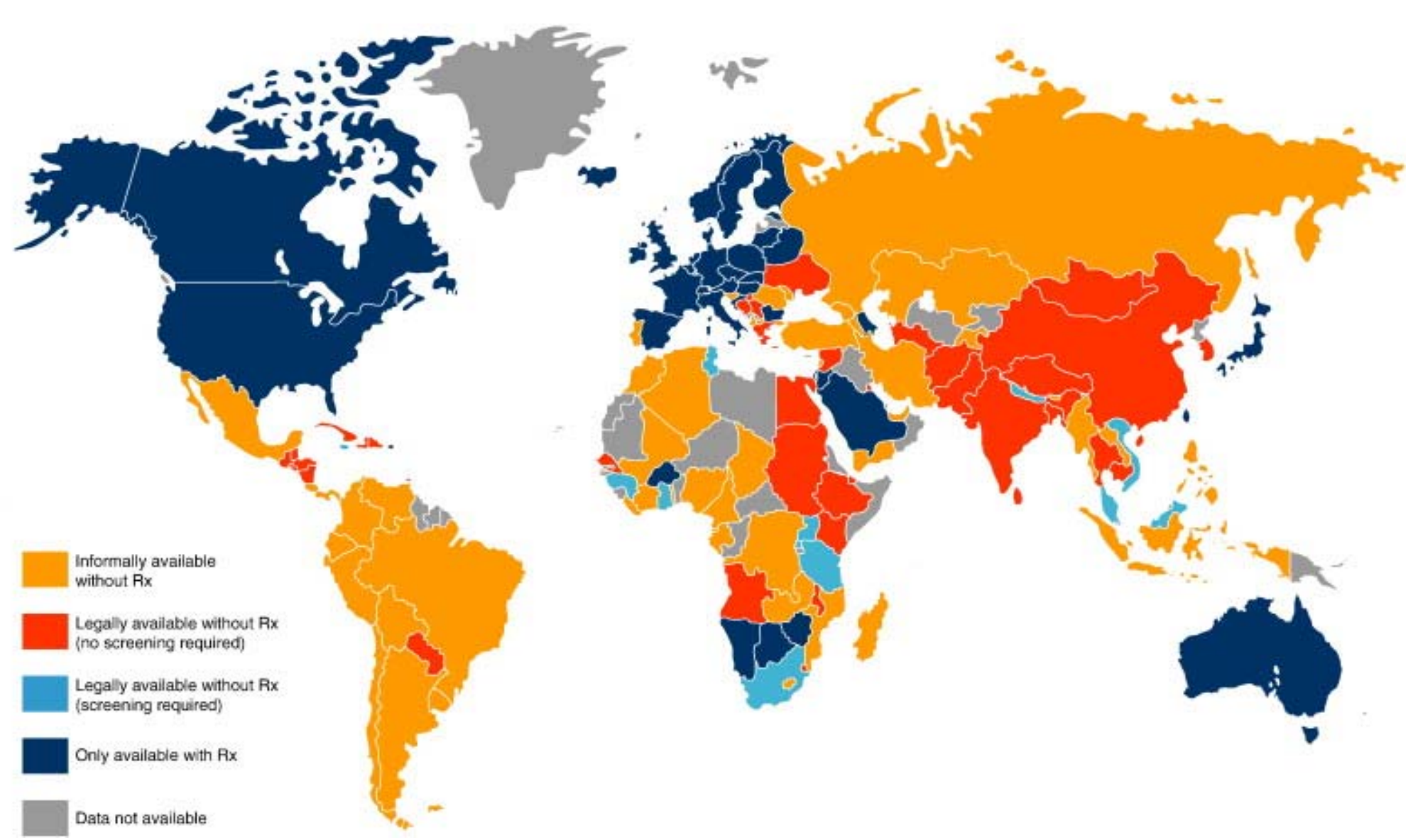


Table 2-1 Global oral contraception prescription and screening requirements: country list (n=147) (Reproduced from Grindlay et al 2013)

\begin{tabular}{|c|c|c|c|c|c|c|}
\hline \multicolumn{2}{|c|}{ Informally available without prescription } & \multicolumn{2}{|c|}{$\begin{array}{l}\text { Legally available without } \\
\text { prescription (no screening required) }\end{array}$} & \multirow{2}{*}{$\begin{array}{l}\text { Legally } \\
\text { available } \\
\text { without } \\
\text { prescription } \\
\text { (screening } \\
\text { required) } \\
\text { Ghana }\end{array}$} & \multicolumn{2}{|c|}{ Only available with prescription } \\
\hline Albania & Liberia & Afghanistan & Kenya & & Australia & $\begin{array}{l}\text { Micronesia, } \\
\text { Federated States } \\
\text { of }\end{array}$ \\
\hline Algeria & Macedonia & Angola & Kuwait & Guinea & Austria & Namibia \\
\hline Argentina & Madagascar & Bangladesh & Malawi & Jamaica & Azerbaijan & Netherlands \\
\hline Armenia & Mali & $\begin{array}{l}\text { Bosnia and } \\
\text { Herzegovina }\end{array}$ & Nicaragua & Malaysia & Belarus & New Zealand \\
\hline Aruba & Mexico & Cambodia & Pakistan & Nepal & Belgium & Norway \\
\hline Bahamas & Moldova & China & Paraguay & Rwanda & Botswana & Poland \\
\hline Bahrain & Mongolia & Cuba & Senegal & South Africa & $\begin{array}{l}\text { British Virgin } \\
\text { Islands }\end{array}$ & Puerto Rico \\
\hline Bhutan & Morocco & Djibouti & Serbia & Tanzania & Bulgaria & Saudi Arabia \\
\hline Bolivia & Mozambique & $\begin{array}{l}\text { Dominican } \\
\text { Republic }\end{array}$ & South Korea & Tunisia & Burkina Faso & Singapore \\
\hline Brazil & Myanmar & Egypt & Sri Lanka & Uganda & Canada & $\begin{array}{l}\text { Slovakia (Slovak } \\
\text { Republic) }\end{array}$ \\
\hline Cameroon & $\begin{array}{l}\text { Netherlands } \\
\text { Antilles }\end{array}$ & El Salvador & Sudan & Vietnam & Cape Verde & Spain \\
\hline Chad & Nigeria & Ethiopia & Swaziland & Ghana & Croatia & Sweden \\
\hline Chile & Palestine & Greece & Syria & Guinea & $\begin{array}{l}\text { Czech } \\
\text { Republic }\end{array}$ & Switzerland \\
\hline Colombia & Panama & Guatemala & Thailand & Jamaica & Denmark & $\begin{array}{l}\text { Taiwan (Republic of } \\
\text { China) }\end{array}$ \\
\hline
\end{tabular}




\begin{tabular}{|c|c|c|c|c|c|c|}
\hline Costa Rica & Peru & Haiti & $\begin{array}{l}\text { Trinidad and } \\
\text { Tobago }\end{array}$ & Malaysia & Estonia & United Kingdom \\
\hline $\begin{array}{l}\text { Democratic Republic of the } \\
\text { Congo }\end{array}$ & Philippines & Honduras & Turkmenistan & Nepal & Finland & United States \\
\hline Ecuador & Portugal & Hong Kong & Ukraine & & France & Zimbabwe \\
\hline Equatorial Guinea & Romania & India & & & Germany & \\
\hline Gabon & $\begin{array}{l}\text { Russian } \\
\text { Federation }\end{array}$ & & & & Hungary & \\
\hline Georgia & Slovenia & & & & Iceland & \\
\hline Grenada & Tajikistan & & & & Ireland & \\
\hline Indonesia & Togo & & & & Israel & \\
\hline Iran & Turkey & & & & Italy & \\
\hline Ivory Coast & United Arab & & & & Japan & \\
\hline & Emirates & & & & Jordon & \\
\hline Kazakhstan & Uruguay & & & & Lithuania & \\
\hline Laos & Venezuela & & & & Malta & \\
\hline Lebanon & Yemen & & & & Marshall & \\
\hline Lesotho & Zambia & & & & Islands & \\
\hline
\end{tabular}


Much of the available literature focuses on whether regularly taken hormonal contraceptives (as opposed to emergency hormonal contraception) should be available from a pharmacy without a medical prescription, either "behind the counter" (subject to some restrictions on age, gender and status) or "over the counter" (not subject to any such restrictions). This research is largely conducted in countries where contraceptives are not available over the counter. There are a large number of surveys and some qualitative data seeking the opinion of users, potential users, pharmacists and health professionals. There is a variety of literature evaluating the current pharmacist delivery of emergency hormonal contraception. There are a number of papers on pharmacists already offering hormonal contraception without a prescription in pilot projects, and some ways to explore partnerships between community pharmacies and healthcare professionals in order to improve patients' sexual health and increase access to contraception.

\subsection{Pharmacists' thoughts about their role in dispensing contraception}

A number of studies have examined pharmacists' thoughts about expanding their role regarding contraception - usually asking pharmacists their willingness to dispense hormonal contraception on a regular basis without a doctor's prescription. Research from a variety of (resource rich) countries shows an enthusiastic response, indicating pharmacists embracing the idea of expanding their role, and welcoming a potential increase in status and autonomy.

Surveys from 145 pharmacists across Canada, and qualitative interviews with a representative 19 demonstrated that $90 \%$ of pharmacists felt they had the skills for assessment, examination, decision-making and follow-up involved in independent prescribing of hormonal contraception. In addition they felt that doing so would give them more pride and autonomy and increase job satisfaction (Norman et al., 2015). A survey of student pharmacists in California, USA in 2003 demonstrated a willingness to provide novel reproductive health services, with $96 \%$ interested in providing hormonal contraception (the pill, patch or ring) when asked. Participants felt that pharmacists providing more services would benefit patients by giving them improved 
access and opportunities for advice about hormonal contraception (Rafie and ElIbiary, 2011). Of 1250 pharmacists in Quebec, Canada, 99.3\% believed they should be 'very involved' or 'involved' in the provision of emergency contraception, with 97.4\% believing they should be 'very involved' or 'involved' in the provision of contraception in general (Laliberté et al., 2012). A survey completed by 2,725 pharmacists in the United States (with only a 19\% response rate) showed that most respondents were comfortable and interested in providing direct access to hormonal contraception in the pharmacy (Landau et al., 2009). The main weakness of the research outlined above is that it asks people how enthusiastic they are about a proposed idea. Such information is of value when considering setting up new services but is not a guide to how likely those services are to be provided in practice, given the varied financial, political and practical elements involved in service expansion, particularly in the field of reproductive health. Of note in the Quebec survey, only $67.8 \%$ described themselves as currently 'involved' or 'very involved' in sexual health activities, with lack of time, space, skills and human resources all cited as barriers to providing more comprehensive services in the pharmacy (Laliberté et al., 2012). These findings were similar to those of Paluck et al, who surveyed 625 pharmacists in British Columbia, Canada. Counselling clients on sexual health aspects of health such as "STDs" and "AIDS" accounted for a very small portion of pharmacists' workload, with $31 \%$ and $41 \%$ of pharmacists never doing this (Paluck et al., 1994). However this does not offer any evidence about their ability or willingness to offer such services, only that in the current set up they are not doing so.

Pharmacists in North-East Scotland expressed a willingness to provide various services in the future, with $75 \%$ open to providing oral contraceptives under a PGD. However only $33 \%$ were willing to provide long-acting reversible contraceptive (LARC) methods (Gale and Watson, 2011). A report by the Scottish Government in 2011 about EHC (and smoking cessation) in pharmacies included 121 surveys with community pharmacists from 13 out of 14 health boards in Scotland. Although pharmacists felt EHC dispensing worked well and needed little improvement, suggestions for improvement did include expanding the service to include pregnancy 
testing and long term contraception, and "new drugs which can be prescribed up to 5 days" (ulipristal acetate) (Hametz et al., 2011). Despite a readiness to provide services pharmacists felt that they lacked adequate training and that pressure of time was a barrier to providing the services they wished to; themes that recur often in the literature (Gale and Watson, 2011; Laliberté et al., 2012; Rafie and El-Ibiary, 2011; Sweeney et al., 2015).

Structured interviews with 20 California physicians and advanced practice clinicians, and a self-administered survey of 482 California physicians and mid-level providers showed that the majority of all types of respondents considered the prescription-only model of accessing hormonal contraception to be unnecessarily restrictive, believing that obtaining contraceptives directly from pharmacists would expand access and increase compliance (Rafie et al., 2012; Rafie et al., 2015). The State of California legislature recently expanded the ability of pharmacists to give "self-administered hormonal contraception" (California State Legislature Assembly, 2013). Although the evidence is that knowledge of this service spread slowly, with few pharmacists aware of it the following year (Richards et al., 2015), two years later enthusiasm seems high among pharmacists, with $71 \%$ of 257 pharmacists reporting they would be likely to expand access to hormonal contraception under the new legislature $(\mathrm{Vu}$ et al., 2015), and over $65 \%$ of student pharmacists in California feeling that they had been adequately educated to offer hormonal contraception (Rafie and El-Ibiary, 2014). Following the California legislation (and similar legislation in Oregon), pharmacists and physicians in Colorado were asked about OTC ('over the counter') access to progestogen-only contraception. There were substantial differences about the different groups' primary concerns, with physicians most concerned about the efficacy of this type of contraception, but pharmacists being concerned about patient self-assessment of contraindications (Yazd et al., 2016).

Interviews with pharmacists in New Zealand who were giving free condoms to 13-24 year olds under a pilot condom card scheme noted generally positive views, though the number of people who had actually used the scheme was low. The pharmacist enthusiasm was based more on their self-perception about their suitability as 
contraception providers rather than interactions with young people (Ryder et al., 2015). Qualitative interviews with pharmacy support staff working for "Healthy Living Pharmacies" in England (a scheme where certain pharmacists proactively promote health by offering advice, services and signposting) showed a mixed response, with some feeling like these pharmacies were useful but others more sceptical, due to concerns over whether patients wanted to "speak to a stranger" over health concerns, and whether there was adequate time and training to provide a good service. Sexual health was felt to be a particularly difficult topic, "due to a perceived discrepancy between the target demographics... compared to regular pharmacy clientele" (Donovan and Paudyal, 2016). Similar reservations were displayed by 273 pharmacists from Northern Ireland who completed self-administered questionnaires. Although generally they were comfortable discussing most aspects of women's health including contraception, $23 \%$ of male pharmacists were embarrassed discussing women's health issues, and $59 \%$ of men and $33 \%$ of women found it difficult to initiate a conversation on women's health issues (McAree and Scott, 2004).

Some qualitative research with groups of patients, GPs and pharmacists in the United Kingdom noted limited enthusiasm among pharmacists for discussion of contraception, with one pharmacist stating that "deciding on which contraception is suitable for the patient is between the patient and their GP”. It is worth noting here that it was the decision-making portion of a discussion about contraception that pharmacists were not comfortable with. They were not asked about delivering contraception to established users. Time pressure was also noted as a barrier to providing a good contraception service (Sweeney et al., 2015).

Pharmacists in Sudan who were filling prescriptions written by a clinician were not asked about expanding their role, but about what they currently did, with $73.4 \%$ noting that they gave information about contraception methods (Mohamed et al., 2013). A small survey of 33 pharmacists in Virginia, USA found almost no support for the idea that contraceptives should be available over the counter (only one person thought pills should be and no pharmacists thought other methods should be), but 
found some support for the idea that methods should be 'behind the counter' available to women aged 17 and older only, with the offer of pharmacist counselling: nine pharmacists believed this about the progestin-only pill, and ten believed that pharmacists should be able to prescribe the progestin-only pill. This research was rare in asking about one of the more effective and long-term methods, the contraceptive injection. Nine pharmacists thought they should be able to prescribe the contraceptive injection; none thought it should be available over the counter (Richard et al., 2015).

Making methods available over the counter does not guarantee availability, perhaps particularly in the United States, where pharmacists may decide not to carry any product based on perceived demand or personal beliefs (Mackin and Clark, 2011). Secondary analysis of data collected for a descriptive study about EHC was conducted in Iowa, US, before and after the US policy change in August 2006 to make oral emergency contraceptives available OTC. They found that the percentage of pharmacies offering EHC increased from 57.8 to $70 \%$, based on 713 pharmacies. However, even after the change availability was only $70 \%$, and myths about safety and method of action persisted (Mackin and Clark, 2011).

\subsubsection{Summary}

In summary, the literature suggests that pharmacists are generally enthusiastic about providing contraceptive services, although more ambivalence is displayed about EHC than regular contraceptives, and more ambivalence about LARC than shortacting methods. This enthusiasm appears to be based on the idea that providing contraceptives is an appropriate role for pharmacies, and one that will enhance their status. However, there has been limited opportunity to see whether this enthusiasm translates into practice, as most pharmacists don't offer hormonal contraception in the UK, US or Canada.

\subsection{Patients' and communities' thoughts about pharmacists dispensing contraception}

As women are able to access regular contraception in only a limited number of countries, multiple papers examined in this section ask patients about their behaviour 
in theory: would they hypothetically attend a pharmacy for contraception? There are undoubtedly difficulties with using research which asks participants what they would do in different circumstances, as there can be no guarantee that they would act as they say or think they would. However, such questionnaires are useful to generally assess feasibility and acceptability of services and are therefore important before spending resources on large trials or expansion of healthcare delivery systems. Intention does not absolutely predict behaviour but there does appear to be a relationship between them (Ajzen, 1991). It is worth drawing the comparison that support for the theoretical availability of EHC from the pharmacist that was demonstrated before this option was available (Folkes et al., 2001) has been reflected in high usage and satisfaction subsequently (Anderson and Blenkinsopp, 2006).

A number of quantitative surveys, largely conducted in the United States, have asked women whether they would attend the community pharmacy to receive their contraception. A telephone survey of 811 women in the United States found that $68 \%$ of women said they would use a pharmacy to access to the oral contraceptive pill, the contraceptive patch and the vaginal ring, as well as EHC. $41 \%$ of women questioned who were not using any contraception stated that pharmacy availability would mean they would start using a hormonal contraceptive (Landau et al., 2006). An online survey of 2046 American women found $62.2 \%$ were strongly or somewhat in favour of the OCP (oral contraceptive pill) being available OTC, and that $28 \%$ of women currently using no method would be likely to use the OCP if available OTC (Grossman et al., 2013). Similar results were found with younger women. 348 women aged 14-17 were recruited to an online survey using social media, and $73 \%$ supported OTC access to oral contraception. $61 \%$ reported that they would likely use oral contraceptives in this way (Manski and Kottke, 2015). It is important to note that barriers exist for this patient population that are not present in the United Kingdom, as demonstrated by the fact that $76 \%$ of women in the telephone survey said they would benefit from not needing to pay for a clinician visit. However other advantages more relevant to the UK included convenient hours $(85 \%)$ and beneficial location (84\%) (Landau et al., 2006). A survey of 651 women attending abortion clinics in urban areas of the US found that although $47 \%$ in favour of OTC access 
stated that this was because it would save money, 63\% were in favour because it would save time (Grindlay et al., 2014).

Fakih et al conducted an online survey with women from Michigan, USA, aged 2526. Only $21 \%(41 / 195)$ had a positive attitude towards pharmacists providing pregnancy prevention information, preferring instead to see a physician (Fakih et al., 2015). Contraceptive information has traditionally been provided by physicians and nurses, so this conclusion is perhaps not surprising. Obtaining advice from a pharmacist would mark a distinct shift in how women have traditionally received this information. There is also the question of how much 'information' patients feel they need when obtaining routine contraception from a healthcare professional. If someone is a well established user of the method then it may not be necessary to provide them with a significant amount of information every time the method is dispensed: this may be an important difference between initial choice and maintenance, and may be relevant to delivery of any contraceptive service from the pharmacist (though there is no literature examining the difference).

Qualitative research in the form of interviews or focus groups provides richer data on how participants feel, and allows the participants themselves to generate ideas. Three pieces of qualitative research have investigated women's ideas about obtaining contraception from their community pharmacist. Focus groups with low-income women in Boston, USA demonstrated general support for OTC access to oral contraception, although women emphasised that they would be unlikely to pay more for OTC access, and advised that the cost should be lower than their current costs. A number of these women reported switching to less effective methods, or from dual to single methods, based on cost (Dennis and Grossman, 2012). The financial factor is less relevant in the UK, where all regularly taken contraception is free regardless of where it is dispensed, and visiting a doctor is also free. Focus groups with young women and women of colour in the US (who may face particular barriers to contraception) echoed the perceived advantages of access, with the main benefit being convenience. Women suggested that this convenience would make it easier to continue pill use, suggesting gaps in use could occur a result of forgetting to make an 
appointment (Baum et al., 2016). In-depth telephone interviews were conducted in August 2015 with 30 women aged 18-19 in California, prior to the new legislation allowing direct pharmacy access for contraception. Almost all participants were supportive of the law, and believed it would lead to better access and increased convenience (Miller et al., 2016). The evidence suggests that women, whether current or potential users, are keen to have the option of obtaining oral contraception from their pharmacist.

A questionnaire sent out to 637 women in Michigan, USA with a follow up rate of $54 \%$, found that $25 \%$ of respondents had had a negative experience with a pharmacist regarding contraception. Examples given of negative experiences are pharmacists not having contraception, or not wanting to dispense it (Higgie et al., 2014). Although numbers and follow up are small, and in addition may reflect the limited amount of contraception currently offered by pharmacist, this research provides evidence against the assumption that it will be preferable and more convenient for all women to visit the pharmacy to fulfil their sexual health needs.

There has been less work outside the United States asking patients about obtaining contraception OTC, and the work that has been done appears to demonstrate more ambivalence. A survey of 488 users of the OCP in the Republic of Ireland found that $87.9 \%$ were in favour of OCP being available without prescription, with convenience and ease of access being the primary advantages (Barlassina, 2015). The Republic of Ireland is a predominantly Catholic country so these findings are perhaps unexpected, but the survey was conducted among current pill users. A survey of 592 pharmacy attenders in Barnet, UK conducted in 1998 is one of the only pieces of literature conducted in the United Kingdom. It found that only $40 \%$ of participants believed it was the pharmacists' role to give health promotion advice (Anderson, 1998 ) - but participants were not asked specifically about contraception, and this may not be something patients traditionally see as health promotion. Of note, $95 \%$ of respondents in this survey agreed that they felt totally at ease when asking the pharmacist for advice and would ask when they needed to do so. It is difficult to 
determine however, whether this would be true of advice relating to contraception and sexual health.

A street survey in an English city centre, aimed at optimising a random selection of people (although people with the ability to get out and about) rather than current pharmacy users, found that $78.9 \%$ of 300 people questioned agreed that contraception should be a pharmacy service. It is unclear however, whether this refers to the current dispensation of prescriptions, or the idea that pharmacists' existing role should be expanded. A further $66.6 \%$ believed that pharmacists should be providing unsolicited advice on sexually transmitted infections. Participants identified barriers to seeking advice from the pharmacy including the pharmacist being too busy, lack of privacy, and doubts about pharmacy expertise. Again, the value of these questions asked in a neutral setting is difficult to quantify. Would those respondents who felt pharmacists should be offering unsolicited advice on STIs for example, be happy to receive such advice themselves? The survey cannot tell us (Krska and Morecroft, 2010).

\subsubsection{Summary}

In summary, the literature from the United States suggests an appetite for access to regular hormonal contraception from the pharmacy. Those questioned believe that pharmacy access will save time, be more convenient, and improve access as a result. This attitude has so far not been demonstrated among patients in the United Kingdom, but that may be because patients have not been asked about this possibility.

\subsection{Emergency hormonal contraception in the pharmacy}

Much of the evidence about the ability of community pharmacists to actually offer contraception is drawn from the provision of emergency hormonal contraception (EHC) to patients without prescription. France was the first country to make EHC available without prescription, in 1999 (Schenk, 2003), and EHC has been available in the United Kingdom without prescription since January 2001 for a cost (Killick and Irving, 2004), and in Scotland without charge since 2008 (Cameron et al., 
2012c). Although professional groups and clinicians were generally in favour of deregulation, there was considerable controversy about deregulation of EHC initially, and challenges were launched (but defeated) in both the House of Commons and House of Lords (Schenk, 2003). Although cost remained (and remains, in England) a barrier, the amount of EHC dispensed by pharmacies increased considerably following deregulation (Marston et al., 2005). Global access remains variable, with 20 countries allowing direct access to EHC OTC, and 76 allowing access from a pharmacist without a prescription (International Consortium for Emergency Contraception, 2017). As EHC has been available from pharmacies in some countries for almost twenty years now, there is a considerable body of literature on the subject, which will be examined here. Unfortunately most of the literature uses surrogate endpoints such as patient satisfaction with the service, pharmacist confidence in delivering EHC, or how quickly patients were able to attend. There is little data about EHC at the pharmacy that is actually powered to detect a reduction in unplanned pregnancy rates (Anderson and Blenkinsopp, 2006; Raine et al., 2005).

\subsubsection{Evidence for EHC in the pharmacy}

As prescription rules about EHC changed, pilot projects in the United Kingdom explored pharmacy dispensing under PGDs (National Institute for Health and Care Excellence (NICE), 2015). Projects in Lambeth and Lewisham, UK and North Yorkshire, UK trained and supported pharmacists to dispense EHC under a specific set of circumstances. Both projects noted the flexibility needed in the PGD as the projects progressed, but the overall success levels with the medication dispensed without difficulty by pharmacists and received by patients, with pharmacy soon accounting for the majority of EHC dispensed (Bacon et al., 2003; Lambeth Lewisham and Southwark Health Action Zone, 2002; Lloyd and Gale, 2005). This was highlighted in an analysis of a large UK multipurpose survey in 2005, which noted no difference in the types of contraception being used by patients since EHC was available OTC - the main difference was that a smaller proportion of women obtained EHC from physicians and a greater number bought it OTC (Marston et al., 2005). 
Similar analysis of general health data from surveys in France (4166 in 1999 and 7490 in 2004) found that the availability of progestogen-only EHC without prescription in 1999 resulted in increased EHC use, but did not increase the numbers of women having intercourse or decrease the age of first intercourse, and did not decrease the numbers of women using the most effective contraceptive methods (though neither did it increase it) (Moreau et al., 2006a). OTC availability did not address health inequalities however; both before and after the change in prescription status, use remained higher among more educated women, and those in cities (Moreau et al., 2006b). Non-prescription status for EHC came to British Columbia, Canada in 2000. Data from prescriptions dispensed from 1996-2003 noted a 102\% increase in EHC received by women compared to a 5 year pre-policy mean. The mean age remained standard and, in keeping with Marston et al in the UK, after the change in prescription status more pharmacies provided EHC than did physicians (Soon et al., 2005). Importantly for service delivery costs, an analysis of patient records of emergency departments in the South-East Thames region of the United Kingdom in 2000-2001 noted that after EHC became available OTC there was a 52\% reduction in the number of women attending emergency departments requesting EHC (M et al., 2004).

One of the earliest initiatives took place in Washington State, USA in 1998-1999, using collaborative prescribing agreements to enable pharmacists to prescribe $\mathrm{EHC}$ directly to women without a prior consultation with a prescriber. This was linked with extensive media outreach and advertising so that patients knew this was possible. Pharmacists dispensed over 1000 EHC pills per month, however feedback on patient satisfaction was minimal, and notably women felt they had received limited information about ongoing contraceptive use. Although the pregnancy and abortion rate dropped following the initiative, the authors note that this is likely due to a number of factors (Gardner et al., 2001; Hayes et al., 2000). A similar project in Ontario, Canada trained pharmacists to dispense EHC with the support of a physician and ran a publicity campaign to alert patients to EHC's pharmacy availability. They found that $98 \%(6931 / 7074)$ pharmacy visits resulted in a prescription, and that patients were very satisfied with the service (based on a response rate of $21 \%$ to a 
satisfaction questionnaire). They estimate that 520 pregnancies were prevented with the pilot project, but this is based purely on a baseline risk and there is no information on the pregnancy rates of women who accessed EHC. Of note also is the $10.2 \%$ who wanted information on ongoing contraception, something they were unable to access from their pharmacist (Dunn et al., 2003). Ragland et al investigated counselling about EHC provided by student pharmacists and found that postcounselling knowledge among the 87 recipients of EHC was higher than precounselling knowledge - although this had fallen with the $43 \%$ who were followed up one to three months afterwards (Ragland et al., 2015).

\subsection{2 'Mystery shopping' for EHC at pharmacies}

The 'mystery shopper technique', or 'simulated client method' is a research method where a researcher acts as a customer to gain real-life data on the practice of a service, without the provider being aware of the researcher's investigative status. In the context of health research, a researcher acts as a patient seeking health services. If the deception is successful, providers' normal method of practice can be observed (Madden et al., 1997). Research using 'mystery shoppers' with pharmacists providing EHC has identified gaps in knowledge among dispensing pharmacists, and/or a failure to discuss ongoing contraceptive methods; this has been demonstrated in Australia (Hussainy et al., 2015; Higgins and Hattingh, 2013; Queddeng et al., 2011), the United States (Wilkinson et al., 2012; French and Kaunitz, 2007; Nelson and Jaime, 2009; Bennett et al., 2003; Sampson et al., 2009; Cleland et al., 2016; Gaffaney et al., 2016; Bullock et al., 2015), Canada (Cohen et al., 2004), Thailand (Ratanajamit and Chongsuvivatwong, 2001; Ratanajamit et al., 2002), Kenya (Liambila et al., 2010), Brazil (Tavares and Foster, 2016), and the United Kingdom (Glasier et al., 2010; Brant et al., 2014). Mystery shopper techniques have also identified other gaps in service provision, for example that EHC is more likely to be procured immediately from an urban than a rural pharmacy in California (Sampson et al., 2009), and Kansas (Samson et al., 2013), and by an English speaker more successfully than a Spanish speaker in California (Sampson et al., 2009). This technique has also been used to identify variations in pharmacies' ability to fill an EHC prescription within 24 hours, with Shacter et al noting that $35 \%$ 
of Atlanta pharmacists were unable to do this, compared to $4 \%$ of Boston pharmacists (Shacter et al., 2007). Brant et al also used this method to identify gaps in pharmacist knowledge in Massachusetts, USA about ulipristal acetate (Brant et al., 2014), and Bullock noted a similar unfamiliarity with ulipristal acetate among pharmacists in Hawaii (Bullock et al., 2015). Legare noted the homogeneity of availability of EHC in different New York neighbourhoods (Legare et al., 2012).

The mystery shopper technique is a useful way to identify problems that particular groups may face: Wilkinson et al used researchers to pose as 17-year-old adolescent females. They found that pharmacists introduced false barriers about EHC, in some cases using these as justification not to dispense the contraception, for example that patients had to be 18 to receive $\mathrm{EHC}$, or that only women could receive it. None of these barriers should exist according to federal guidelines (Wilkinson et al., 2014). The disadvantage of the mystery shopper technique is that it carries a strong risk of confirmation bias, particularly as people conducting the research are often the shoppers themselves. Nevertheless, if a reasonable attempt at standardisation is made, it offers the opportunity to record actual unselfconscious practice from the patient's point of view in an immediate way (Madden et al., 1997). The method is at its most useful when examining more objective outcomes, such as whether a particular intervention is spontaneously mentioned. The research performed in Scotland examining the dispensing of EHC found that ongoing contraception was not mentioned $57 \%$ of the time (Glasier et al., 2010).

\subsubsection{Pharmacist and other healthcare professionals' attitudes to EHC}

A number of different surveys with pharmacists investigate both their attitude to EHC and their experience of dispensing it. A survey of 112 pharmacists in Durban, South Africa in 2001 found that $69 \%$ felt EHC should be available without a prescription, but $60 \%$ felt that increased availability of EHC would decrease the use of barrier contraception (Hariparsad, 2001). 300 community pharmacists in Texas, USA who completed a survey in 2003 displayed reasonable knowledge about EHC, but $27 \%$ of participants were opposed to dispensing it, reasons cited included religious beliefs $(86 \%)$, moral concerns $(81 \%)$ and a belief that use of EHC might 
lead to an increase in unprotected sex (56\%). Although respondents agreed pharmacist-initiated emergency contraception would enhance the role of community pharmacists, they were nevertheless largely unwilling to participate (Griggs and Brown, 2007). A survey of 85 community pharmacists in Rhode Island conducted in 2007 found that $69 \%(n=59)$ strongly disagreed with the statement that EHC should be available OTC in Rhode Island, despite the fact that $59 \%(n=50)$ agreed or strongly agreed with the fact that EHC OTC would reduce unwanted pregnancies in Rhode Island (Orr and Kachur, 2007). A pilot study including a three-hour focus group produced a self-administered questionnaire to send to 1424 registered pharmacies in New Mexico, USA in 2004. Of 523 respondents, only 13\% $(n=67)$ had participated in a training programme for EHC. $56 \%(n=295)$ agreed or strongly agreed with the statement; "I am concerned that oral emergency contraceptives will be used as a regular form of birth control", and $25 \%(n=131)$ with the statement "I think oral emergency contraception is a form of abortion". Write-in statements also revealed strong beliefs among pharmacists that EHC meant the expulsion of a fertilised egg, which they classed as an abortion (Borrego et al., 2006).

Self-completed surveys of 427 pharmacists across Australia in 2008-9, five years after EHC was made available OTC, noted that neither moral restrictions nor the perceived impact of EHC on sexual health influenced pharmacists' decisions to decline provision of EHC (Hussainy et al., 2011). Semi-structured interviews in 2010 with 145 pharmacists and pharmacy managers in and around Cairns, Australia found that most respondents were in favour of OTC provision of EHC and did not have moral or religious concerns. $34 \%$ of pharmacists believed that OTC EHC discouraged regular contraceptive use by young people (Downing et al., 2011).

Focus groups conducted with 14 UK (United Kingdom) pharmacists about the deregulation of EHC in 2001 noted particular attitudes about EHC, notably concerns about the concept of 'abusing' the service available, although no pharmacists could offer examples of this (Seston et al., 2001). These concerns were echoed by 51 pharmacists surveyed in Kuwait, $48 \%$ of whom were concerned that EHC encourages irresponsible behaviour. No pharmacist surveyed stocked it (Ball et al., 
2006). $51.4 \%$ of 367 Puerto Rican pharmacists surveyed in 2006 supported a law that enabled them to dispense EHC without prescriptions, although their knowledge about its mechanism of action was generally lacking (Fuentes and Azize-Vargas, 2009). Of 501 pharmacists in South Dakota, USA interviewed, 84\% did not believe EHC should be available over the counter (Van Riper and Hellerstedt, 2005).

In-depth interviews conducted in the North-East of England with pharmacists offering EHC noted approval by pharmacists, not only of their own role and job description but of what offering EHC could do for the pharmacy profession in general, notably enhancing their status among the public (Bissell and Anderson, 2003) (Bissell et al., 2006). This was echoed by further interviews in 2006 with pharmacists in the North of England. "I think [EHC]'s great. I think it really has enhanced the profession.” (Cooper et al., 2008)

Interestingly, a survey of both GPs and pharmacists conducted in London found that GPs had more knowledge about EHC, but were less prepared to prescribe it. Only $55 \%$ had no reservations about offering EHC, compared to $75 \%$ of pharmacists (D Souza and Bounds, 2001). A self-administered survey in Sweden of both nursemidwives and pharmacy staff who were dispensing EHC found a much higher percentage of nurse midwives giving information on side effects, mechanism of action and efficacy, as well as advising a pregnancy test, than pharmacist dispensers (Aneblom et al., 2004). Contraceptive counselling, including for EHC, takes a level of expertise and confidence that may not be part of the pharmacist's skill set without substantial training and opportunities to practice. These findings reinforce a general theme of pharmacists being enthusiastic about offering sexual health services, but present less evidence for their current skills and knowledge, particularly when compared to other healthcare professionals.

\subsubsection{Timing of access to EHC}

There is some evidence that women find it quicker to access the pharmacy, and are able to take EHC sooner after unprotected sexual intercourse (UPSI) when visiting a pharmacy than when visiting a clinic (Lewington and Marshall, 2006; Black et al., 2008); this is important when effectiveness is inversely proportional to number of 
hours after unprotected sexual intercourse it is taken (World Health Organisation, 1998). However, obtaining EHC is only a surrogate outcome when the actual goal is reducing unplanned pregnancies. There is in fact no good evidence that increased provision of EHC from pharmacies reduces the likelihood of an unplanned pregnancy. A large randomised, single-blind, controlled trial in California divided women into three groups: those who were given EHC in advance, those who could attend a pharmacy for EHC when they needed it, and a control group who attended a clinic providing family planning services when they needed EHC, as was standard practice. Those attending the pharmacy experienced no significant reduction in unplanned pregnancy rates compared to controls. This was a large study, powered for a difference in pregnancy rates. However it did rely on self-reporting of sexual intercourse, and at least in part on self-reporting of pregnancy rates (Raine et al., $2005)$. Further reporting on the same study noted that $66 \%(127 / 194)$ found it very convenient to attend the pharmacy for EHC, compared with $56 \%(36 / 64)$ finding it very convenient to attend the clinic. However, 85\% (258/305) found it very convenient to be provided with EHC in advance, and no option made a difference to unplanned pregnancy rates (Rocca et al., 2007). Another RCT (randomised controlled trial), also powered to detect a difference in unplanned pregnancy rates, noted no difference in pregnancy rates between women given EHC in advance to take when they wanted, and those attending clinics to obtain EHC as per usual practice (Raymond et al., 2006). These limitations are emphasised by a Cochrane Review in 2007, which noted that even advance provision of EHC only improves rates of EHC taken, and not pregnancy rates (Polis et al., 2007), suggesting that even the most minimal time between UPSI and EHC may not reduce unplanned pregnancies significantly. This idea was underlined by a comprehensive review in 2014 of the policy in the USA to expand access to EHC at a state and federal level. Gross et al noted that doing so had had no statistically significant effect on birth or abortion rate - what it had done was shifted the venue in which EHC was provided, and therefore had resulted in some cost savings (Gross et al., 2014). In conclusion improved access to EHC, as offered by pharmacy and even advance provision, may not be as significant an intervention for women as it might at first appear. 


\subsubsection{Ongoing contraception after EHC use}

It has been postulated that the most important intervention at the time of dispensing EHC is not the giving of the medication itself, but the opportunity to improve contraception use, either by increasing knowledge about the method already being used, or directing the recipient towards more effective methods (Black et al., 2008). Clinical guidance in the $\mathrm{UK}$ is that when a woman is given $\mathrm{EHC}$, ongoing contraception should be discussed with all women, including typical use failure rates and the benefits of LARC over short-acting methods (Faculty of Sexual and Reproductive Healthcare, 2012). By definition those who are attending for EHC are a patient population who are not using a regular form of contraception correctly. Interviews with 232 women in Ghana when they were obtaining EHC found that over $40 \%$ used EHC as their main method of contraception and yet had sex at least twice a week (Chin-Quee et al., 2012). There is some evidence that a clinic, rather than a pharmacy setting, is more likely to discuss ongoing contraception with patients, and that this information is more likely to be correct. An observational study of 133 women in London found that when completing questionnaires four months after obtaining EHC, only $28 \%$ of those attending a pharmacy could remember a discussion of future contraception, compared to $90.4 \%$ who had attended a clinic. Although this method is obviously subject to the difficulties associated with remembering a consultation at a later date, there is no reason to think that the memories of the groups would differ significantly, and therefore recall bias should generally apply equally to both groups (Black et al., 2008). A self-administered survey of 126 adolescents who attended the pharmacy for EHC in Washington, USA, found that among $31 \%$ needing new birth control, $21 \%$ had no intention of seeing a doctor in the next month (Sucato et al., 2001). However it is difficult to draw conclusions about what counselling they received from the pharmacist, or what they might have done had the pharmacy service not been available. Surveys from 309 pharmacists participating in this research showed that two thirds of them had referred at least one patient receiving EHC for ongoing medical care - but there is no record of whether they attended (Sommers et al., 2001). Qualitative interviews with users of EHC from the pharmacy by Bissell and Anderson found that some patients felt the 
service should be confined to supplying EHC, rather than discussing long-term contraception: "You'd go elsewhere for that." (Bissell and Anderson, 2003)

A small number of pilot projects have attempted to link prescription of EHC to prescription of ongoing contraception. A study in Lambeth, UK, produced a PGD for pharmacists who had undergone additional training to supply oral contraceptives without prescription. Of 741 consultations about contraception, $45.5 \%$ (274) were made as a result of discussion during EHC prescription. 69\% resulted in the supplying of oral contraceptives to either an established or first time pill user. Patient questionnaires revealed that patients were satisfied with this service, and both 'mystery shoppers' and clinical audit demonstrated that pharmacists adhered to the PGD. However, of crucial importance in preventing unplanned pregnancy is the fact that only $1.2 \%$ of contraceptive consultations resulted in a referral for LARC (Parsons et al., 2013). A pilot study in Edinburgh, Scotland, randomised 168 women presenting for $\mathrm{EHC}$ to either standard care, receiving one packet of the progestogenonly pill (giving them one month to arrange ongoing contraception), or an invitation to present the empty packet of EHC to a family planning clinic for a rapid access appointment. At six to eight weeks post-EHC 56\% of women who had received POP were using an effective method of contraception, compared to just $16 \%$ who had received standard care (though the follow up period was extremely short) (Michie et al., 2014). So there is some evidence of success in contraception uptake when pharmacists manage to link provision of EHC to ongoing contraception. An RCT with 938 women in Jamaica who were not regular contraception users but who attended a pharmacy for EHC, randomised women to receive either normal care, or a coupon for $3-27 \%$ off the price of oral contraceptive pills purchased from the pharmacy. Within the 6 month period $15 \%$ of participants had adopted either the OCP, injectable or IUD as a regular method, but there was no significant difference between the trial arms (Chin-Quee et al., 2010). Although prescription of long-term contraception is not an endpoint in itself, it is at least more likely to lead to prevention of unplanned pregnancy for a longer period than a one-off prescription of EHC. Nevertheless, it must be remembered that the important outcome, which was 
not examined in any literature linking EHC to ongoing contraceptive provision, is a reduction in unplanned pregnancy.

\subsubsection{Patients' experience of EHC from pharmacies}

As EHC has been available from the pharmacy for a number of years, there is a body of research asking patients directly about their experience. In a survey of adolescents in Washington State who had obtained EHC from a pharmacy, only 58\% stated that they would have attended a doctor if they couldn't get emergency contraception from a pharmacist. Over $90 \%$ were happy with the level of information and privacy, and would recommend the service to a friend (Sucato et al., 2001). This is echoed by similar research from the United Kingdom. From 53 completed surveys from young people (average age 19.6 years) accessing EHC in the pharmacy, 41.5\% $(n=22)$ stated that if they could not get EHC from the pharmacy they would wait and see if they got pregnant. $18 \%(\mathrm{n}=10)$ chose a community pharmacy for ease of access (Berry et al., 2016). Although there is insufficient evidence to conclude with certainty that some women would attend the pharmacist for regular ongoing contraception who would not attend a doctor or nurse, these papers provide some evidence for this idea. A survey of 426 Californian women accessing EHC at the pharmacy found that they had chosen this because they believed pharmacy access was faster (54\%) and more convenient (47\%) than attending a physician (Greene et al., 2006). However, focus groups with women in Australia about provision of EHC OTC found that, although women were happy about this development, they did not wish to have information about contraception or sexually transmitted infections delivered by pharmacists, largely because they believed that to be the role of the GP or family planning doctor (Hobbs et al., 2009).

5020 questionnaires were distributed in community pharmacies in Manchester, Salford and Trafford. 430 were returned (a $9 \%$ response rate). $93 \%$ of respondents said they found it easy or very easy to obtain EHC, and $99 \%$ were satisfied or very satisfied with how their request was dealt with (Anderson et al., 2001). Focus groups as part of the same evaluation showed that participants found receiving EHC from the pharmacy convenient, discrete and straightforward. They did discuss the 
embarrassment about having to attend for $\mathrm{EHC}$, and the emphasis on being a 'responsible' user, for example taking it after contraceptive failure (Bissell and Anderson, 2003). These concerns are perhaps less likely to be reflected in patients receiving regular contraceptive supplies from community pharmacy who may feel this reflects more 'responsible' behaviour. Focus groups with women in Australia who had received EHC from a pharmacy found both positive and negative experiences; although women felt that EHC should be available, a number reported concerns about how much privacy was available in the pharmacy setting (Hobbs et al., 2009). Focus groups with women from France, Norway, Sweden and Portugal identified some national differences. Although women were generally happy with the pharmacy dispensing EHC, opinions varied between nationalities about how much of an active role the pharmacist should play. Women from France felt that it was important that they ask the same questions a doctor would, whereas women from Norway displayed a reluctance to engage in discussion with their pharmacist. Women from all countries expressed doubts about the amount of knowledge pharmacists had about EHC (Gainer et al., 2003). Conversely, 419 women in the UK completing anonymous questionnaires after receiving EHC at the pharmacy stated that they were more likely to be satisfied with information about side effects from their pharmacist, compared to their doctor (91\% versus 58\%). As this was a sample of women who had chosen to attend the pharmacy, rather than a more random sample as in Gainer et al, this preference for pharmacy is perhaps unsurprising (Killick and Irving, 2004).

531 adolescents from 49 states in the US who had engaged in unprotected intercourse completed an online survey, after recruitment through social media and adolescent-oriented websites. Those living in states with pharmacist access to EHC were much more likely to have obtained it without a prescription, and those who obtained EHC in a pharmacist-access state were more likely to be satisfied with their experience (Rubin et al., 2011). A survey of 800 randomly selected women in Sweden found that $78 \%$ believed that access to EHC over the counter was positive, although only $27 \%$ of respondents had used it in this context (Larsson et al., 2004). In contrast, Seston et al conducted a stated preference discrete choice experiment 
with 269 women attending primary care clinics in the North West of England, examining women's preferences and trade-offs for aspects such as opening hours, staff seen and length of wait. They found that respondents preferred to be seen by a doctor or a nurse to a pharmacist, and that this choice was one of the most important factors for them (others being attitude of staff and privacy of consultation) (Seston et al., 2007). If women are dissatisfied with aspects of a service, they may choose not to visit it and risk unplanned pregnancy.

Patients were also satisfied with the pilot projects whereby ongoing contraception was prescribed after EHC (Parsons et al., 2013), though women in the Scottish study voiced some concerns, notably that they went away with questions that they didn't feel able to ask the pharmacist, and that they were unsure whether it was the role of the pharmacist to undertake contraception consultations (Michie et al., 2016). However a small study of 86 women in the USA who had received EHC noted that the majority were very satisfied with the pharmacist counselling, with $93 \%$ agreeing that the counselling would enable them to use EHC in the future. (Battle et al., 2013). (In both cases the low recall of patients about EHC at one to three months after counselling in Ragland's study is worth remembering here (Ragland et al., 2015).) Importantly, patient satisfaction is only another surrogate outcome. It is useful as an assessment of healthcare services currently offered, but it cannot tell us about how valuable the service is, when the objective must be a reduction in unplanned pregnancies at a population level. The research already mentioned, casting doubt on the fact that rapid access reduces unplanned pregnancy rates (Raine et al., 2005; Raymond et al., 2007; Polis et al., 2007), is crucially relevant here, though is rarely mentioned in discussion of broadening access to EHC via pharmacy provision (Department of Health, 2005).

\subsubsection{Summary}

In summary, pharmacists in a number of countries currently successfully deliver EHC. Women can obtain it more quickly than they would if they attended a clinic, and report a good experience obtaining EHC from the pharmacy. This was the conclusion of a structured literature review in 2006 (Anderson and Blenkinsopp, 
2006). However, the evidence is that this rapid access does not reduce unplanned pregnancy rates (Polis et al., 2007). This may be in part because discussion about ongoing contraception is limited after pharmacy dispensation of EHC. Preliminary research examining the potential of pharmacists to dispense or advise about ongoing contraception choices has not yet demonstrated the long-term efficacy of this strategy. Pharmacy access to EHC is clearly quicker, but other aspects of care such as discussion of the method and ongoing contraception may still favour a clinical setting.

\subsection{Ongoing contraception services delivered or facilitated by the pharmacy}

A small number of pilot projects have examined pharmacist provision of contraception that has not already been prescribed by a doctor. The project in Lambeth, UK has already been discussed in the context of EHC: five community pharmacists who had undergone additional training provided oral contraceptive pills via a PGD. An audit showed PGD adherence, and questionnaires demonstrated high levels of customer satisfaction. However $93 \%$ of patients left with oral contraceptive pills, and only $1.2 \%$ were referred for a more effective LARC method (Parsons et al., 2013). The Direct Access study took place in Washington, USA from 2003-2005, and gave women the option of accessing combined hormonal pills, patches and rings from their pharmacist. 195 women were prescribed hormonal contraceptives, $70 \%$ of which were still using the method after 12 months (follow-up rate 65\%). Agreement between women's self-assessment and nurse practitioner assessment for risk factors was over $90 \% .97 .7 \%$ of participants stated that they were satisfied or very satisfied with obtaining hormonal contraception from the pharmacist, and at $97 \%$ would feel comfortable continuing to do so (Gardner et al., 2008). A pilot RCT in North Carolina, USA investigated women receiving their contraceptive injection from the community pharmacy: a more effective method than the contraceptive pills trialled in Lambeth. Women were randomised to receive two injections either from the clinic that recruited them or from a trained community pharmacist. There was no significant difference in patient satisfaction with location, convenience, privacy or provider respect among either group. However although follow up rates were small 
$(36 \%, n=9$, in the pharmacy group and $48 \%, n=12$, in the clinic group for the second injection), the relative risk of returning to the pharmacy compared to the clinic for the second injection was 0.73 , and for the third injection 0.75 . The small study demonstrated feasibility, but may indicate that women, at least initially, prefer to return to clinic than attend community pharmacy for contraceptive injections (Picardo and Ferreri, 2010).

The Picardo study took place in a setting where contraception incurs costs, and also used one pharmacy 1.5 miles away from the original Planned Parenthood clinic. One of the advantages of community pharmacies compared to clinics, cited by both patients and pharmacists, is direct access (Anderson and Blenkinsopp, 2006; Anderson and Thornley, 2014; Barlassina, 2015; Bissell and Anderson, 2003; Bissell et al., 2006; Black et al., 2008; Saramunee et al., 2014). It may be more useful therefore, to provide patients with options for community pharmacies in a wide variety of locations, in the explicit hope that they would be able to receive their contraceptive injection nearer to their work, home or where they shopped, and therefore increase convenience. This variety was present in a similar intervention in California, where 27 pharmacists in 26 independent and chain pharmacies partnered with 19 clinics/physician offices. Over a two year programme, established users of the contraceptive injection DMPA-IM (intra-muscular depot medroxyprogesterone acetate) were given the option of attending a trained participating pharmacist for their repeat injections. 69 women received their injections at the pharmacy, several (number not specified) returning five or six times. Unfortunately there is no data about how many women were offered this service and chose not to use it, or how many were eligible and not offered it. The success of the project seems to have been due in part to the enthusiasm of two pharmacists, one in particular who developed excellent links with his local physicians (Monastersky and Landau, 2006).

A similar project was developed in Uganda, where 139 DSOs 'drug shop operators' (not necessarily trained pharmacists, but staff working in a drug shop) were trained to administer the DMPA-IM injection to patients. A drug shop (also known as a patent medicine vendor shop, similar to a UK pharmacy) is mandated to sell only 
unrestricted and unclassified medicines - which would include oral contraceptives but not the contraceptive injection. However, in practice drug shops often do provide injections. Interviews with 585 recipients during the project showed that $57 \%$ of women interviewed had received their last injection elsewhere and had therefore switched to receive their injection at the drug shop. Reasons mentioned were convenient location (43\%), shorter waiting times (12\%) and flexible opening hours (11\%). 98\% of those interviewed intended to use the drug shop as the location of their next injection (Akol et al., 2014). Surveys in Nigeria showed that, although it is not currently legal for drug shops to offer injectable contraceptives, $14.9 \%$ of 396 DSOs who completed the survey reported administering them. Out of 393 women surveyed, $19.6 \%$ had received an injection from a DSO, and $67 \%$ rated the service as good (Ajuwon et al., 2013). By contrast evidence from Ghana from 2014 however, suggests that DSOs sell and give information about the contraceptive injection, but none of the 94 DSOs studied had given the contraceptive injection, necessitating patients to obtain their prescription and then attend a healthcare clinic at a further visit (Lebetkin et al., 2014). In the context of sub-Saharan Africa, receiving contraceptive injections from DSOs, few of whom are trained pharmacists, is well received by women. A technical consultation in 2013 reviewed the evidence and concluded that, with appropriate training and monitoring, DSOs can effectively and safely administer contraceptive injections, and that this should be reflected in national and international family planning standards and policies (Stanback et al., 2014).

The introduction of the Affordable Care Act (ACA) in 2010 in the United States affected the way some women accessed contraception, with a greater number of women receiving a prescription from family planning providers, which they then filled at a pharmacy, rather than receiving the contraception from the family planning provider. Focus groups conducted in Massachusetts, USA noted that women responded positively to this change, noting the ease of filling prescriptions at the pharmacy, the convenience of locations being nearer to their home, and the professionalism of pharmacists asked about contraception (McIntosh et al., 2012). 
Although regular hormonal contraception is not available OTC in the USA or the UK, it is available elsewhere. A survey of 1502 women from Kuwait, where it is possible to buy OCPs OTC found that $25 \%$ of women used OCPs without physician consultation. The paper notes that women attending the pharmacy for OCP were rarely told about how to use the pill or side effects, but it must be noted that this data is based on patients' recall of information (Shah et al., 2001). Some women in the United States also have the option of hormonal contraception over the counter: women who live near the U.S./Mexican border can get contraceptive pills OTC from Mexico. A 2010 study of 532 clinic users and 514 pharmacy users found that among pharmacy users, for $27 \%$ of those surveyed, avoidance of having to get a doctor's prescription was a determining factor (Potter et al., 2010). Further research with the same cohort also showed that obtaining contraception OTC meant patients were more likely to continue using the method (Potter et al., 2011).

Concern has been expressed in the literature that obtaining contraception OTC would mean a reduced screening rate for patients for cervical changes or breast abnormalities, with fewer reasons to attend a clinic. However a study comparing 514 US women who received their contraception OTC in Mexico with 532 women who received it from a family planning clinic in the US, noted no difference in screening rates for STIs, cervical smears or breast exams (Hopkins et al., 2012). By contrast a study using data from the 2000 Mexican National Health Survey, a large door-todoor survey collecting information on blood pressure, smoking and contraceptive use from women aged 20 and over, found that $10 \%$ of pill users under 35 and $33 \%$ of pill users over 35 had health conditions that were relative or absolute contra-indications to pill use, according the WHO medical eligibility criteria (Yeatman et al., 2006). If contraception were available over the counter the reliance would be on both patients and pharmacists to identify exclusion criteria. Shotorbani et al paired up 399 women completing a self-administered questionnaire and healthcare providers, and found that participant-provider agreement was over $90 \%$ (Shotorbani et al., 2006). Research using the mystery shopper technique in Kingston, Jamaica, found that although in most cases pharmacists correctly denied the OCP to women in whom it was contraindicated (11/15 interactions with a heavy smoker and 14/15 interactions 
with a hypertensive woman), they also denied it in 10/15 interactions with an eligible adolescent, suggesting that OTC access is safe, but may not eliminate all barriers to access (Chin-Quee et al., 2006). Grossman recruited women at shopping venues and asked them whether they thought they were eligible for the contraceptive pill: only $6.6 \%$ of 1,271 participants thought they were eligible when they weren't, largely due to undiagnosed hypertension (Grossman et al., 2008). An expert review of the evidence in 2011 concluded that compelling evidence exists about the safety and effectiveness of providing oral contraception OTC, and that this is reflected in a public perception that this would be safe (Grossman, 2011).

\subsubsection{Partnership working}

The White Paper 'Choosing health through pharmacy' asserts that pharmacies should "work in partnership with health organisations and the wider public health community across primary, community, social care and hospital settings' (Department of Health, 2005). However, although it is certainly a reasonable hypothesis that partnerships between pharmacy and the wider community, and with GP practices and sexual health centres in particular, are a useful way to involve pharmacists in improving health behaviours, there is only very limited evidence that such partnerships deliver a high standard of patient care. The White Paper acknowledges this deficit, saying that this is because, in a developing area of public health "the available evidence, as generated from research studies, will inevitably lag behind the reality of good practice and service innovation" (Department of Health, 2005). Unfortunately, over a decade later, there remains a paucity of research on delivering sexual health through partnerships with community pharmacy. A novel service in Glasgow, Scotland set up a sexual health service in the premises of a busy community pharmacy (McAllister et al., 2002). The service was staffed by a nurse or health promotion officer, so did not expand or increase the pharmacists' role, though it is possible that services being in such close proximity to one another would expand the pharmacists' education through informal information exchange. The service was felt to be moderately successful, although numbers were initially small. A further paper about the same service notes the difficulties inherent in partnership working between the health and commercial sectors (Mackie et al., 2002). 
In the North-East of England, educational seminars were provided for pharmacists giving EHC that emphasised the efficacy of a copper IUD (intrauterine device) at preventing unplanned pregnancy, and a straightforward 'fast-track' pathway was developed for pharmacists to use to refer women to their local sexual health centre. This change in education resulted in a 2.73 fold increase in the number of women at the sexual health centre having a copper IUD fitted for emergency purposes, with the majority being referred by a pharmacist (Clement and Mansour, 2014). This example of partnership working may well have been so successful due to its specific and measurable outcome: increasing the knowledge of pharmacists' about the efficacy of the copper IUD, and providing easier access for them to refer patients. In addition, whilst not looking at unplanned pregnancy rates, this important intervention increased the uptake of one of the most effective methods, both of emergency and ongoing contraception (Trussell, 2011). The California intervention by Monastersky et al, where physicians could refer patients for repeat contraceptive injections at participating pharmacies, does seem to have worked much more smoothly when one pharmacist worked closely with his physician colleagues, an example of clinician/pharmacist partnership working to deliver good contraceptive care (Monastersky and Landau, 2006).

\subsubsection{Summary}

A number of different approaches have demonstrated modest success in the involvement of pharmacists in contraception care, though none have used a reduction in unplanned pregnancy as an endpoint. Delivery of the contraceptive injection by DSOs in Sub-Saharan Africa is useful, and small studies from the USA have suggested that this may be a service pharmacists can successfully deliver. Other ways of partnership working such as placing healthcare professionals within a pharmacy, or close fast-track links to enable pharmacists to refer patients for the copper IUD, also seem to work. Studies on a larger scale with each of these approaches would provide more information. 


\subsection{Long Acting Reversible Contraception (LARC) at the pharmacy}

The overwhelming majority of studies investigating contraception from the pharmacy, either in theory or in practice, concentrate on short-acting methods such as the pill, the patch and the ring, or on EHC. There is almost no literature that investigates the possibility of patients receiving LARC from their community pharmacy. The studies mentioned in section 2.5 investigating the contraceptive injection at the pharmacy are the only ones (Picardo and Ferreri, 2010; Monastersky and Landau, 2006; Akol et al., 2014; Ajuwon et al., 2013). However, longer acting methods are known to be more effective at preventing unplanned pregnancy (Trussell, 2011; Winner et al., 2012). Another successful intervention is the development of a pathway for pharmacists to refer patients to clinicians for emergency IUDs: an example of presentation to the pharmacy resulting in patients obtaining a LARC method as well as having access to the most effective emergency method (Clement and Mansour, 2014). Although pharmacists have rarely been asked about delivering LARC, a survey of 94 community pharmacists in Scotland noted only $33 \%(n=31)$ interested in "the administration of long acting reversible contraceptives (e.g. by injection)" (Gale and Watson, 2011).

\subsection{The need for further research}

Before a substantial amount of public money is used to equip pharmacies to deliver contraception there is an urgent need for well-designed research examining the best and most effective approaches. Ideally, quantitative research looking at access to contraception, including emergency contraception, should use an endpoint most relevant to patients and the community, i.e. a reduction in unplanned pregnancy rates. This might compare rates in a group using the novel access method versus a control, or at least compared to similar unplanned pregnancy rates in the relevant population group. Surrogate endpoints such as access to contraception must be acknowledged as such, and ideally studies should be powered to detect pregnancy rates. 
The enthusiasm in the literature for pharmacists to offer regular contraception without a prescription is not echoed in government literature (Department of Health, 2005). Despite debates about whether regular hormonal contraception should be offered in the pharmacy existing in British medical journals almost ten years ago (Grossman, 2008; Jarvis, 2008), there is no evidence in the literature of any growing support for this idea in the UK. If the medication is to be offered OTC then certain criteria must be met: 1) the patient must recognise and diagnose the condition in themselves, 2) they must be able to assess the information about the medicine correctly, 3) the medicine must be as effective as when used as recommended and 4) the drug must be safe (Brass, 2001). Although each of these conditions seems easily fulfilled by making contraception over the counter, crucially the short-acting methods which most of the literature concentrates on are not as effective as LARC methods at preventing unplanned pregnancy.

\subsection{The literature review in the context of this thesis}

The contraceptive injection has traditionally been a method requiring patients to attend a healthcare professional every 12-13 weeks. Although some would classify it as a LARC method, users need to visit a HCP (healthcare professional) more often than with other shorter-acting methods such as the OCP, because current guidance is to offer users a year's supply if they are unlikely to change methods in a year (Faculty of Sexual and Reproductive Healthcare, 2016). In addition, there is no reminder to patients, such as using the last pill or patch, which encourages them to book a further appointment (Davidson et al., 1997). Frequency of having to attend a healthcare provider may be part of the reason why discontinuation method of this method is high when compared to other methods (Davidson et al., 1997; Paul et al., 1997; Kaunitz et al., 2014). The contraceptive injection is therefore a method where receiving an injection from an available and convenient provider, such as a community pharmacist, seems particularly appropriate. Trials with involved, committed pharmacists such as the one seen in California seem to have the best evidence (though still very scant) for pharmacists contributing effectively to contraception uptake by patients (Monastersky and Landau, 2006). There is scope to examine the feasibility of community pharmacists delivering contraceptive injections 
in other countries and settings. (Of note a healthcare professional used to be required to give this method, though a subcutaneous form became licensed for self-injection in September 2015 (Faculty of Sexual and Reproductive Healthcare, 2015a).)

Before embarking on a definitive randomised controlled trial, there would need to be initial work exploring feasibility, especially given the lack of conclusive evidence of success in the research already done. This would require a pilot study, and perhaps some locally based patient research to look at accessibility. These will be examined in upcoming chapters. 


\section{Chapter 3 Background research for a pilot study examining contraceptive injection at the pharmacy: a patient survey}

\subsection{Introduction}

Chapter 2 examined the evidence available for delivering contraception from the community pharmacy setting. There is enthusiasm for the concept in United Kingdom governmental policy (Department of Health, 2005) and, as detailed in Chapter 2.3, the majority of women questioned in the United States think that contraceptive services should be available from their pharmacy. Most pharmacists themselves are also open to the idea, as shown in Chapter 2.2. However, evidence actually assessing services delivering contraception from the pharmacy is far from conclusive. In addition the majority of the literature concentrates on short-acting contraceptive methods such as the contraceptive pill (Shah et al., 2001; Potter et al., 2010; Parsons et al., 2013). Other methods such as the contraceptive injection, implant and intrauterine device are more effective at preventing unplanned pregnancy (Trussell, 2011; Winner et al., 2012). There is a small amount of evidence that delivery of the contraceptive injection from the community pharmacy is appealing to a group of patients, though no study has shown that it improves unplanned pregnancy rates (Monastersky and Landau, 2006; Picardo and Ferreri, 2010; Maderas et al., 2008). The typical failure rate for the contraceptive injection is $6 \%$, compared to $9 \%$ for the more popular oral contraceptive pill (Trussell, 2011), so increasing uptake of this method among patients using less effective methods could reduce unplanned pregnancy rates.

\subsection{Justification for the methodology}

Although there is very limited literature about delivery of LARC from community pharmacies, a review of the literature suggests there may be some potential for delivery of the contraceptive injection. The location of pharmacists within the community, particularly communities of deprivation, as well as longer opening hours than many general practices, makes them a place that is easily accessible for people. 
If women could receive the injection from the pharmacy this might reduce barriers to regular injections, improving continuity and potentially uptake of this effective method. However, before proceeding with any research, even an initial feasibility study, it was important to examine the willingness of our patient population to attend the community pharmacy for contraceptive injections. Although this would seem to give patients a convenient way of accessing one of the more effective types of contraception, there is limited evidence about this practice, and none from the United Kingdom. As a preliminary step, we decided to therefore conduct a survey among our patients, to assess their readiness to attend. This would ensure that conducting research with this population was feasible, and that recruitment would be possible.

We conducted a survey with patients attending a large urban sexual health centre in Edinburgh, exploring their feelings about attending the community pharmacy to receive the contraceptive injection.

\subsection{Patients' preparedness to attend a pharmacy for contraceptive injections: a survey}

Published in full: Heller R, Cameron ST. Evaluating the attractiveness of the availability of injectable progestogen contraceptives at the community pharmacy setting in the United Kingdom. International Journal of Pharmacy Practice 2016 Aug;24(4):247-52. Published with permission of the editor and co-author.

\subsubsection{Introduction}

There is growing recognition of the potential that community pharmacists could play in delivering sexual health services within the UK. Pharmacies are located directly in communities and have extended evening and weekend hours. Examples of sexual health services delivered successfully by community pharmacists include provision of emergency contraception (EC) (Black et al., 2008; Marston et al., 2005), and treatment of partners of individuals with uncomplicated Chlamydia infection (Cameron et al., 2010). In addition there have been pilot projects of provision of the combined oral contraceptive pill from the pharmacy (Parsons et al., 2013), and pharmacists providing 'bridging contraception' (a one month supply of a 
progestogen-only pill) alongside oral EC (Michie et al., 2014). A pharmacist in the UK has also been trained to fit contraceptive implants and give the DMPA-IM injection (Pharmaceutical Journal News Team, 2011). Community pharmacists in the UK currently administer other subcutaneous injections and are essential for delivery of national flu vaccination programmes (Hind et al., 2004; Brown et al., 2012; Anderson and Thornley, 2014). Pharmacists administer these medications under a Patient Group Directive, a legal framework that allows certain registered health professionals to administer specified medicines to a pre-defined group of patients without the necessity of seeing a doctor. Patient Group Directives were first introduced through legislation in 2000, and are currently covered under The Human Medicines Regulation 2012 (National Institute for Health and Care Excellence (NICE), 2015). This questionnaire was administered in Scotland, where sexual health services differ somewhat at an administrative level from other parts of the UK. However the same 'no cost' services to the public are provided (through the National Health Service) including contraceptive methods.

The contraceptive injection depot medroxyprogesterone acetate (DMPA) is given every 12 weeks, but discontinuation rates of this contraceptive method have been noted to be particularly high (Davidson et al., 1997; Paul et al., 1997; Potter et al., 1997). In one study, discontinuation rate due to dissatisfaction was $42.3 \%$ (Moreau et al., 2007). Although not the only factor, remembering to return to clinic every three months, as well as the inconvenience of having to do so has been cited by users as a contributory factor to discontinuation (Davidson et al., 1997). A subcutaneous formulation of injectable contraception (Sayana Press ${ }^{\circledR}$, Pfizer Inc.) is now available in the UK. This preparation is a lower dose (104mg compared with $150 \mathrm{mg}$ for IMDMPA) but is similar in other aspects including efficacy, injection interval, return to fertility and bleeding pattern (Faculty of Sexual and Reproductive Healthcare, 2013). The subcutaneous preparation comes packaged in a simple delivery system known as uniject, consisting of a polyethylene reservoir pre-filled with micronised DMPA and an integral fine subcutaneous needle. This may facilitate administration of injectable contraception and so expand the range of health professionals who can administer it, including community pharmacists. Self-administration of subcutaneous DMPA has 
been shown to be feasible in several trials (Cameron et al., 2012b; Prabhakaran and Sweet, 2012; Williams et al., 2013), and Sayana Press ${ }^{\circledR}$ has recently been licensed in the United Kingdom (UK) for this purpose (Faculty of Sexual and Reproductive Healthcare, 2015a).

For women that are already established users of the contraceptive injection, receiving repeat injections from their community pharmacy may be an attractive option. Administration of subcutaneous DMPA by community pharmacists (using a more complicated system of syringe and needle) has already been shown to be feasible in pilot studies conducted in the United States (Picardo and Ferreri, 2010; Monastersky and Landau, 2006).

We wanted to establish whether the option of receiving the injectable contraceptive from a community pharmacist was acceptable to women, particularly in the case of those who are using less effective methods.

\subsubsection{Methods}

From 3rd February to 11th July 2014, an anonymous, self-administered questionnaire was offered to women attending a single-site walk-in clinic at an integrated sexual and reproductive health (SRH) service located in the centre of Edinburgh, UK. The site was selected as it sees a high volume of patients and, as research is often conducted from this centre, reception staff are used to handing out research materials to patients. Women attend this walk-in clinic largely for contraception and sexually transmitted infection screening. Clinic reception staff were asked to hand out a questionnaire to all women of reproductive age (16 to 50 years) attending the clinic, to complete while they were waiting to be called to see the doctor or nurse. Women attending the termination of pregnancy (TOP) service or the menopause clinic were not invited to participate. Women were asked to place completed questionnaires in a closed collection box.

Questionnaires were piloted with five service users and consisted of a brief written introduction that outlined the injectable method of contraception and introduced the idea that it might be possible to receive injections at a community pharmacy in the 
future. It was explained to women that the injection would be subcutaneous ("under the skin rather than into the muscle"). Questions required simple tick-box responses, with a space to expand upon responses if wished. Questionnaires asked women if they were current, previous or never users of the injectable, which method of contraception they were currently using, and whether availability of the injectable from a local pharmacist would influence their decision to use this method. They were also asked to select perceived advantages or disadvantages of receiving their contraception from the community pharmacy from a predefined list of options.

\subsubsection{Data analysis}

All data was entered into Microsoft Excel for analysis. All questionnaire responses were entered including those where not all questions had been answered. Responses to questions were expressed as numbers and percentages, with percentages rounded to the nearest integer. Questions were analysed separately, i.e. if a questionnaire was not fully answered analysis was performed on the questions that were answered.

The local Quality Improvement Team for sexual and reproductive health approved the project. The scientific officer of the local ethics committee reviewed the project proposal and confirmed that ethical approval was not required.

\subsubsection{Results}

During the study period 1605 women attended appointments at the walk-in clinic. The number of women attending is likely to be smaller as some women may have attended more than once. In theory all of these women may have received a questionnaire although the reception staff handing out the questionnaires confessed to usually forgetting - so this is unlikely. Unfortunately due to a high workload for the staff handing out the questionnaires we do not know how many women were offered a questionnaires and declined. 240 questionnaires were distributed and 220 were returned (91\%). 163 were fully completed (74\%) and the remaining 57 were partially completed. 3 only had information completed about the contraception being used by the participant, and 54 had answers completed stating whether they would attend the pharmacist for the contraceptive injection but no reasons completed why/why not. 
The main contraceptive methods being used by respondents are outlined in table 3.1. As a percentage of 220 returned questionnaires there were 8 current users of the injectable in the women surveyed (4\%), 23 previous users $(10 \%)$ and 187 who had never used it (85\%). Of those that had never used it, 33 (18\%) had considered using it in the past but decided against it.

Table 3-1 Contraceptive method in use by participants

\begin{tabular}{|l|l|l|}
\hline $\begin{array}{l}\text { Method in use at time of completing } \\
\text { questionnaire* }\end{array}$ & $\begin{array}{l}\mathrm{n}(\%) \\
\mathrm{n}=220\end{array}$ & $\begin{array}{l}\text { n (\% of users } \\
\text { of this method) } \\
\text { that would } \\
\text { consider a } \\
\text { contraceptive } \\
\text { injection from } \\
\text { a community } \\
\text { pharmacist } \\
\mathrm{n}=65\end{array}$ \\
\hline $\begin{array}{l}\text { Combined oral contraception } \\
\text { (pill/patch/ring) }\end{array}$ & $60(27 \%)$ & $15(25 \%)$ \\
\hline Condoms & $43(20 \%)$ & $12(28 \%)$ \\
\hline Not using any contraception & $34(16 \%)$ & $8(24 \%)$ \\
\hline Contraceptive implant & $30(14 \%)$ & $16(53 \%)$ \\
\hline IUD/Mirena IUS & $27(12 \%)$ & $11(41 \%)$ \\
\hline Progestogen only pill & $9(4 \%)$ & $2(22 \%)$ \\
\hline Current depot users & $8(4 \%)$ & $1(13 \%)$ \\
\hline Withdrawal method & $2(1 \%)$ & 0 \\
\hline Contraception is not necessary for me & $3(1 \%)$ & 0 \\
\hline Trying for a pregnancy & $1(0.5 \%)$ & 0 \\
\hline Diaphragm & $1(0.5 \%)$ & 0 \\
\hline Vasectomy & $1(0.5 \%)$ & 0 \\
\hline * for respondents using more than one method, most effective method \\
being used
\end{tabular}

Of 23 previous users, the most common reason for having stopped the injectable was irregular bleeding, cited by 10 women ( $44 \%) .6$ respondents $(26 \%)$ indicated that having to see a doctor or nurse every 3 months was a reason for stopping this contraceptive method. Of the 33 women who had considered using the contraceptive injection but decided against it, 24 (73\%) cited having to see a doctor or nurse every 
3 months as one of the reasons they had decided not to use it. Other reasons are further detailed in 3.2

Table 3-2 Reasons for stopping the contraceptive injection, or deciding not to use it

\begin{tabular}{|l|l|}
\hline Reasons for stopping (completed by 23 ex-users of this method) \\
\hline Reason & $\begin{array}{l}\text { n (no of times ticked) } \\
(\%) \text { (percentage of respondents } \\
\text { selecting it*) }\end{array}$ \\
\hline Irregular bleeding & $10(44 \%)$ \\
\hline Weight gain & $6(26 \%)$ \\
\hline Acne & $6(26 \%)$ \\
\hline $\begin{array}{l}\text { Having to see a nurse or doctor every } 12 \\
\text { weeks }\end{array}$ & $6(26 \%)$ \\
\hline $\begin{array}{l}\text { Reasons for deciding not to use the method (completed by 33 women who } \\
\text { had considered using the contraceptive injection but decided not to) }\end{array}$ \\
\hline Worry about weight gain & $28(85 \%)$ \\
\hline $\begin{array}{l}\text { Having to see a doctor or nurse every } 3 \\
\text { months }\end{array}$ & $24(73 \%)$ \\
\hline $\begin{array}{l}\text { Don't want a method where your period } \\
\text { stops }\end{array}$ & $22(67 \%)$ \\
\hline Delay in return to fertility & $15(45 \%)$ \\
\hline Worry about bone health & $14(42 \%)$ \\
\hline Prefer something more long acting & $14(42 \%)$ \\
\hline Other people put me off & $9(27 \%)$ \\
\hline Not familiar with this method & $8(24 \%)$ \\
\hline
\end{tabular}

Out of 212 current non-users, 65 (31\%) said they would consider using the contraceptive injection if they could get this from their local pharmacy. 58\% of them $(n=37)$ were using less effective methods or no methods. The methods that all 65 were using are in table 3.1. In response to why this would be a good option for them, most respondents ticked more than one option from a list. The main reasons were quicker appointments (52\%) and easier access (47\%) Further reasons are detailed in table 3.3. Just one current contraceptive injection user said that they would be interested in getting future contraceptive injections from a community pharmacy. 
143 respondents $(65 \%)$ said that they would not consider using the contraceptive injection even if it could be given by a community pharmacist. The reasons largely related to the method itself rather than the place of delivery. $53(37 \%)$ said that they did not wish to use this method at all, and 32 respondents (22\%) ticked 'other' and stated that they wanted a hormone free method. Only $11(8 \%)$ stated that they would prefer to see a nurse or doctor. Reasons are further outlined in table 3.3. We did not include cost as a factor because in the United Kingdom all contraception is free to users, as is seeking advice from a pharmacist, doctor or nurse. Obtaining contraception from a pharmacist would therefore represent no financial advantage or disadvantage to patients.

Table 3-3 Perceived advantages and disadvantages of receiving contraceptive injections from a pharmacist in the community

\begin{tabular}{|c|c|}
\hline \multicolumn{2}{|c|}{$\begin{array}{l}\text { Perceived disadvantages (completed by } 143 \text { respondents who would not } \\
\text { choose this option) }\end{array}$} \\
\hline Perceived disadvantages & $\begin{array}{l}\mathrm{n}(\text { no of times ticked) } \\
(\%) \text { (percentage of respondents } \\
\text { selecting it*) }\end{array}$ \\
\hline $\begin{array}{l}\text { Would not consider using this method } \\
\text { ever }\end{array}$ & $53(37 \%)$ \\
\hline Other: "want hormone free method" & $32(22 \%)$ \\
\hline Other reasons given** & $25(17 \%)$ \\
\hline $\begin{array}{l}\text { Prefer to see a nurse or doctor every } 12 \\
\text { weeks }\end{array}$ & $11(8 \%)$ \\
\hline Prefer coming to this clinic & $7(5 \%)$ \\
\hline $\begin{array}{l}\text { Prefer an injection into the muscle to } \\
\text { under the skin }\end{array}$ & $4(3 \%)$ \\
\hline Do not trust a pharmacist to give it & $0(0 \%)$ \\
\hline Did not answer & $47(33 \%)$ \\
\hline \multicolumn{2}{|c|}{$\begin{array}{l}\text { Perceived benefits (completed by } 65 \text { respondents who indicated interest in } \\
\text { this service) }\end{array}$} \\
\hline Perceived benefits & $\begin{array}{l}\mathrm{n}(\text { no of times ticked) } \\
(\%) \text { (percentage of respondents } \\
\text { selecting it*) }\end{array}$ \\
\hline Better opening hours & $25(38 \%)$ \\
\hline Easier to get to & $31(47 \%)$ \\
\hline Closer to my home & $20(30 \%)$ \\
\hline Quicker appointment & $34(52 \%)$ \\
\hline
\end{tabular}




\begin{tabular}{|l|l|}
\hline Prefer an injection under the skin & $14(21 \%)$ \\
\hline Did not answer & $17(26 \%)$ \\
\hline * Percentages add up to >100 as most respondents selected more than one \\
option.
\end{tabular}

\subsubsection{Discussion}

Our survey suggests that almost one in three women who is not currently a user of the contraceptive injection would consider using this method if they could receive injections from their local pharmacy. Quicker appointments, better opening hours and the pharmacy being easier to get to were all stated as reasons why women thought this would be a good option for them.

Questions examined only what people said they might do in theory, so it is possible that this may not truly reflect the proportion of women who would choose to go to the pharmacist for contraceptive injections if this were a current option. We also captured only a small number of current users. This may be an indication that regular users of DMPA are choosing to attend their GP or practice nurse, rather than a sexual and reproductive health clinic. It may also reflect the local service policy of actively encouraging women who need a repeat injection to attend their local GP practice for this, so that SRH consultations can be prioritised for individuals with more complex sexual health needs. It does mean however that the survey findings cannot estimate what proportion of total current users would switch to go a pharmacy if this option was available. It would be worthwhile repeating this survey in a primary care setting to see if results differ. Since we relied on reception staff handing out questionnaires to women, only a small proportion of women that were eligible were handed questionnaires. We did not capture information on demographics of respondents. Due to busy clinics and a high workload reception staff were unable to keep track of how many eligible women were not offered questionnaires. The list of possible advantages and disadvantages of receiving contraception at the pharmacy were 
created based on what we anticipated women's views would be and not on qualitative research with users.

Interestingly, a quarter of ex-users stated that having to visit a doctor or nurse every three months had been a reason that led to them stopping this method of contraception, suggesting that some women might continue using the contraceptive injection if they did not have to make a regular appointment with a doctor or nurse. In keeping with the existing literature, the main reason cited for stopping was problematic bleeding (Davidson et al., 1997; Paul et al., 1997; Potter et al., 1997). Among the women that had given consideration to using the contraceptive injection in the past, but elected not to use it, almost three quarters of respondents stated that the fact that you had to see a doctor or nurse regularly had been a factor in them deciding not to use it. This gives a strong indication that removing this aspect of the contraceptive injection might make it a more attractive option to women who are considering using this as their method of contraception.

Although almost one in three women indicated that they would be interested in the contraceptive injection if the option to receive it from pharmacies were available, this may be an overestimation of the proportion of women choosing this if available. When asked about the reason they had decided not to use, or stopped using, the contraceptive injection, respondents mainly cited many reasons unrelated to delivery of the contraception, notably irregular bleeding and weight gain. As with all contraception, women's decision to use a method is likely to be influenced by a number of factors, and perceived disadvantages may be more or less relevant when weighed up against perceived advantages. Our survey does seem to suggest that availability at a pharmacy would be a perceived advantage to a fair proportion of women who are using less effective methods. Very few women stated that they preferred to see a doctor or nurse or attend a clinic.

This research captures patients' opinions on a novel potential option for delivering contraceptive injections in the community and demonstrates that a significant proportion of women would welcome the possibility of receiving this method from a community pharmacy. The availability of contraception injections from a local 
pharmacy would increase access for women and might make this longer acting method more attractive to a greater number of women, notably to women who are put off by the necessity of regular appointments with a doctor or nurse, who may opt instead for less effective methods. It may also mean that women might be less likely to discontinue the method once they start using it and this could potentially prevent more unintended pregnancies. Although a pilot study of pharmacists administering the contraceptive injectable in the US did not observe any difference in continuation rates of the method compared to standard practice, the study was a pilot of just fifty women randomised to clinic or pharmacy (Picardo and Ferreri, 2010). The study did confirm that women found the pharmacy convenient, that pharmacists were respectful and overall women were very satisfied with receiving the injectable from this setting.

Studies indicate that both providers and women prefer a subcutaneous injection of DMPA delivered via Sayana Press ${ }^{\circledR}$ 's uniject system to an intra-muscular injection DMPA (Burke et al., 2014b; Burke et al., 2014a). Whilst self administration of the subcutaneous DMPA remains highly acceptable to women (Cameron et al., 2012b; Prabhakaran and Sweet, 2012; Williams et al., 2013), the option of receiving injections from pharmacists nevertheless expands the options for increasing access to this method and may be particularly attractive to women who don't want to selfadminister injections.

Our survey did not specify whether injections from the community pharmacy would be repeat or first injections of this method. It would make sense for a pilot project to explore continuation injections from the pharmacy, following method initiation from a clinician.

\subsubsection{Conclusions}

Our survey indicates that for a significant proportion of women who are not using the contraceptive injection, the ability to receive repeat injections at a local pharmacy would be an attractive prospect. This could be a simple strategy that might increase uptake and continuation rates of injectable contraception, and reduce unplanned pregnancies. 
Further research piloting pharmacists as providers of this method of contraception is therefore warranted. Our survey did not specify whether injections from the community pharmacy would be repeat or first injections of this method. It would make sense for a pilot project to explore continuation injections from the pharmacy, following method initiation from a clinician.

\section{$\sim$ End of published paper}

\subsection{Conclusion}

Both the literature review presented in Chapter 2 and the survey presented in this chapter set out the background exploration that was done before embarking upon a pilot project delivering the contraceptive injection from community pharmacies. Although most of the evidence in the literature review is from the United States, it suggests acceptability among a wide variety of patient populations, citing convenience and time saved as possible advantages to attending the pharmacy.

This chapter describes a survey undertaken with Edinburgh patients, to examine their willingness to attend a community pharmacist to receive the contraceptive injection. It is reassuring that patients appear to trust their pharmacist to give their contraceptive injection. This echoes other research published about influenza vaccines given by pharmacists. Questionnaires completed by 898 patients in Scotland as part of a pharmacy influenza vaccination scheme, demonstrated similar views, with $99.8 \%$ of respondents $(n=896)$ answering that the injection was administered as well as by other health care professionals (Hind and Downie, 2006). Although this is the only evidence published on patients' willingness to attend their pharmacy for a contraceptive injection, there are no documented concerns about receiving other injections from a pharmacy, and no documentation from patients that this procedure should only be undertaken by a doctor or a nurse.

The last large national survey of contraceptive use was published in 2009. At this point 3\% of women aged 16-49 in the United Kingdom were using the contraceptive injection as their contraceptive. It was most popular among the more fertile age groups, with $9 \%$ of 25-29 year olds who used contraception using the injection and 
$6 \%$ of 20-24 year olds (Lader, 2009). Given these numbers, if only a small number of women chose to attend a pharmacy for their contraceptive injection, the reduction in visits to clinicians in an overstretched healthcare system would be significant.

Importantly, our survey suggests that a number of patients currently using other, less effective methods of contraception might change to the more effective injection if the convenience of pharmacy availability was possible. These responses provide some evidence for our hypothesis that pharmacy availability could improve continuity and uptake of this method, and suggest that recruitment will be possible for a pilot trial. Although there may still be as yet unidentified barriers to this practice, patient acceptability is unlikely to be a significant one.

The background research will help to inform the methodology design of the pilot study, as outlined in chapter 4 . 


\section{Chapter 4 Delivering injectable contraception at the community pharmacy: research background and methodology}

This chapter sets out the background for the pilot study, outlines the process by which the study was conducted, and explains the research methodology. The identification of both pharmacy and patient participants is outlined then the processes of recruitment, training and documentation are explained.

\subsection{Justification for the research}

As has been outlined, the DMPA contraceptive injection (or Depo Provera ${ }^{\circledR}$ ) is an effective method when users are able to receive regular doses at the correct time intervals (Faculty of Sexual and Reproductive Healthcare, 2016). However, discontinuation rates are high compared to other effective methods (Kaunitz et al., 2014; Trussell, 2011), with one population study showing that only the cervical cap or diaphragm and the contraceptive sponge (both among the least effective methods), had higher discontinuation rates than Depo-Provera ${ }^{\circledR}$, in which the discontinuation rate due to dissatisfaction was $42.3 \%$ (Moreau et al., 2007). An observational study using a primary care database noted that users of the contraceptive injection were the least likely to use just that method in one year, compared to users of all other methods (Cea Soriano et al., 2014a). Although the primary reason for discontinuing the contraceptive injection is usually given as experiencing side effects (Potter et al., 1997; Paul et al., 1997), the inconvenience of having to attend a healthcare provider may be a contributory factor, and the balance of positive and negative factors may change according to how convenient a method is. A study of users of DepoProvera ${ }^{\circledR}$ not long after FDA approval in the US noted a 17\% rate of "default discontinuers", who failed to return for further injections - this could be due to the time burden of returning to clinic (although in the UA cost may also be a factor) (Potter et al., 1997). Compared to either LARC methods (which last for years) or short-acting methods (where the advice is to give patient's a year's supply at a time (Faculty of Sexual and Reproductive Healthcare, 2016)), using the contraceptive 
injection in its traditional form entails more time spent visiting a healthcare provider than any other method.

Research conducted with our own patient population as outlined in Chapter 3, supports the idea that this may contribute to discontinuation rates, with $26 \%$ of exusers identifying regular visits to a healthcare provider as the reason that they stopped using the method. This research also showed that $31 \%$ of non-users of the injection would potentially be interested in receiving contraception injections from the community pharmacy (Heller and Cameron, 2016).

\subsection{Background to the research hypothesis}

My hypothesis was that offering women contraceptive injections from a variety of community pharmacies would increase accessibility to the method, which would increase continuation rates, and ultimately decrease the unplanned pregnancy rate among users. If accessing this contraceptive method was more straightforward, it might be more attractive to users of other, less effective methods. The most robust way to test these hypotheses would be an RCT, the highest standard of evidence about scientific practice. A suitable design would be a two-armed study where women were randomised to receive contraceptive injections from primary care or sexual health clinics as per standard practice, or to receive contraceptive injections from a pharmacy. A variety of pharmacists would enable participants to choose one with relative proximity to them. Evidence from the literature would suggest that under current service models, $65 \%$ of women discontinue the contraceptive injection in less than 24 months (Paul et al., 1997). It would be reasonable therefore to continue this trial for three years. This would enable women to decide to continue or discontinue as they wished, for two years, and then be followed up for a further year. In order to produce the highest level of evidence, an RCT would be powered to detect a difference in unplanned pregnancy rates. However, this would be extremely challenging, as numbers of unplanned pregnancies are likely to be small. Evidence from primary care suggests that the majority of women who change from injectable contraceptives switch to oral contraceptives (Cea Soriano et al., 2014a), the typical failure rate of which is approximately $9 \%$ over the first year of use, compared to a 
failure rate for the contraceptive injection of 3\% (Trussell, 2011). Therefore even people discontinuing injectable contraceptives are likely to have low rates of unplanned pregnancy (although higher than those who continue it). An RCT powered to detect a change in unplanned pregnancy rates would therefore be extremely large, which is one of the reasons so few studies use this endpoint. A very large RCT over a number of years would be extremely resource intensive. Before proceeding with such an RCT it is therefore good practice to perform a pilot study to determine the recruitment and consent rate, establish the acceptability of the service and identify appropriate outcome measures (Lancaster et al., 2004).

Two small studies in the United States have already examined the feasibility and acceptability of administering the subcutaneous contraceptive injection in the pharmacy setting. One in North Carolina randomly allocated 50 women to receive either their usual follow up contraceptive injections at Planned Parenthood, or at a pharmacy close by. They found recruitment straightforward, but noted that women receiving injections at the pharmacy were no more likely to return for follow up injections than were women who received injections at Planned Parenthood (Picardo and Ferreri, 2010). A demonstration programme in California gave established contraceptive injection users the option of attending a participating pharmacist for repeat injections. The experience was a mixed one. Some pharmacists developed excellent links with patients and the clinic, but the research emphasises the provider support necessary to make the program run successfully (Monastersky and Landau, 2006). The question of feasibility and acceptability in a UK setting has not been addressed, crucially in a setting where all contraception and healthcare delivery is free. The reasons for carrying out this particular pilot study were therefore to determine how easy it would be to recruit patients for this service, to examine acceptability and feasibility both for pharmacists and patients, and to identify any barriers in place to both research and service delivery in this field.

The research question of self-administration was not addressed in the study design, as at the time the research was conceived self-administration of the contraceptive injection did not have a product licence in the UK, despite studies showing its high 
levels of patient satisfaction and feasibility (Keith et al., 2014). In September 2015, whilst the research was being conducted, DMPA-SC (Sayana Press ${ }^{\circledR}$ ) became licensed for self-injection (Faculty of Sexual and Reproductive Healthcare, 2015a).

\subsection{Designing the pilot study}

The US pilot study used a community pharmacy near the clinic that recruited patients were attending (Picardo and Ferreri, 2010). However, my belief was that a significant advantage offered by community pharmacies was their proximity to patients, and therefore their easier accessibility and greater convenience. To establish this I therefore planned to select a number of pharmacists across the city, so that patients would be able to receive contraceptive injections from a pharmacist near them. It was not a component of the research that patients should be offered a choice of contraception at the pharmacy, as the assessment of feasibility was being made for this method only. Where pharmacists have demonstrated more ambivalence towards offering contraception, it has been about making the choice of contraceptive with patients (Sweeney et al., 2015). In addition, the precedent set for other contraceptives that require repetition, such as the contraceptive pill and patch, is a visit to a healthcare professional at least annually (Faculty of Sexual and Reproductive Healthcare, 2016). The choice was therefore made to offer only repeat contraceptive injections at the pharmacy, after research staff introduced women to the method in a clinical setting. Participants would receive three injections from a pharmacist, and then re-attend a nurse or doctor to discuss their contraceptive choice, sexual health, and make any changes to their care as necessary. This timescale was validated during the life of the research, when the guidelines for self-injection using Sayana Press ${ }^{\circledR}$ were produced by the Faculty of Sexual and Reproductive Healthcare, and stated that recipients should see a doctor yearly (Faculty of Sexual and Reproductive Healthcare, 2015a).

\subsection{Identification and recruitment of pharmacies}

Recruitment of pharmacists was not conducted according to an a priori hypothesis, as there was nothing in the literature to guide this research. The aim instead was to choose pharmacists whom we thought would be able to work productively with the 
research team, who had an interest in providing the service, and who would be able to offer convenient and straightforward access to as many participants as possible. In order to identify pharmacies that were usefully located, the postcodes of all patients who had attended sexual health clinics in Edinburgh and West Lothian for the contraceptive injection over the two years before the project started were identified. All patients who had received two or more contraceptive injections were then mapped by their residential postcode. Postcode areas in Edinburgh and West Lothian of high-density contraceptive injection use were therefore identified (figure 4.1). Finally Edinburgh and West Lothian pharmacies were then identified in areas of high-density contraceptive injection use. As patients attending our service for contraceptive injection came from these places, it seemed likely that recruitment would be most successful here.

A number of pharmacists throughout Edinburgh and West Lothian already participate in the 'partnership voucher scheme', a scheme where partners of patients positive for Chlamydia trachomatis can exchange a voucher for $1 \mathrm{~g}$ of azithromycin at a community pharmacy (Cameron et al., 2010). Pharmacists involved in this scheme had established partnership working with our sexual health service and were familiar with sending paperwork there for remuneration. In addition they had already shown a level of enthusiasm for participating in novel service development in the field of sexual health, 
Figure 4-1 Contraceptive injection use mapped by postcode with pharmacies in Edinburgh

Depo Provera injections by Lothian Sexual Health Services 2013

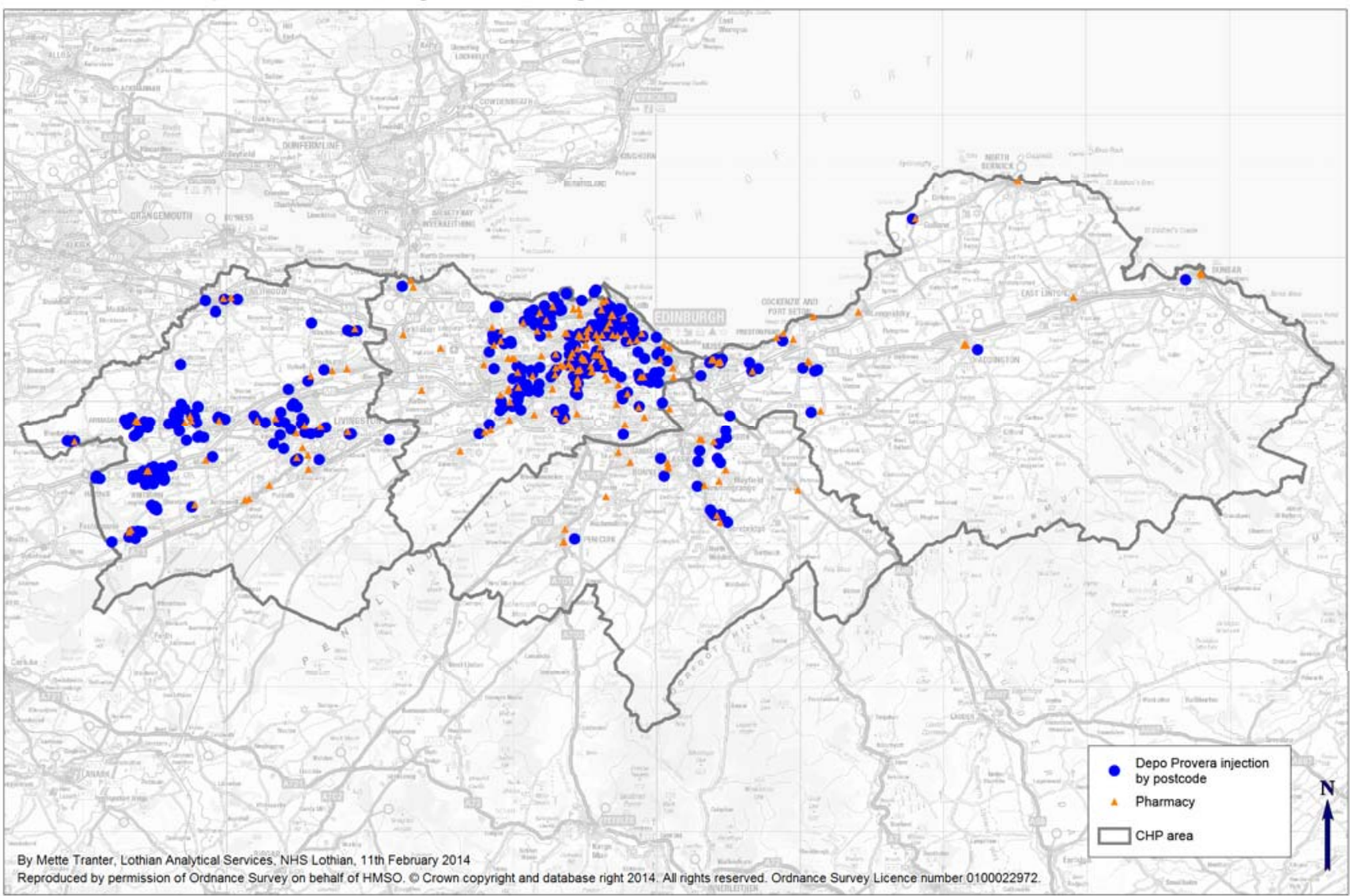


Twelve pharmacies were initially chosen based on their participation in the Chlamydia voucher scheme and their proximity to numbers of users of the contraceptive injection (figure 4.1). Priority was also given to pharmacies that were based in large shopping centres, based on the idea that attending these might be particularly convenient. All identified pharmacists were invited to attend an information evening to learn more about the project. Representatives from all pharmacies attended the event which consisted of information about Sayana Press ${ }^{\circledR}$ and a demonstration on how to give the injection. The role of the pharmacists involved was outlined, and information from attendees was sought about the running of the research. After discussion and consultation pharmacists felt that participants telephoning in advance to arrange their injection would be most feasible.

Following this evening everyone who had attended was contacted and asked if they would like to be involved. Two pharmacists opted out, citing pressure of work $(n=1)$ or feeling the project was not suitable for their premises $(n=1)$. The other ten wanted to be involved with the project and this was felt to be a suitable number to proceed with. It was important to achieve a balance so that women had a choice of pharmacists to attend and yet each pharmacist gave a reasonable number of injections to maintain competence. A further pharmacist who was not originally invited to attend but who has worked closely with the team on other research pertaining to sexual health was also invited to participate, and responded enthusiastically. With this number involved, the pharmacies involved were mapped, and a good area of the city seemed to be covered. Recruitment of pharmacists therefore ended.

\subsection{Training of pharmacists}

A patient group direction (PGD) had already been created in NHS Lothian, enabling pharmacists to give the subcutaneous contraceptive injection without a doctor's prescription. This PGD identified who was eligible to receive the contraceptive injection from pharmacists, and necessitated pharmacists using it being familiar with the exclusion and inclusion criteria for the contraceptive injection, as well as side effects and risks of delivery. All pharmacists involved were trained in a) giving of 
subcutaneous injections (necessary to participate in the project) and b) anaphylaxis training (arrived at after discussion within the research team and discussion with the pharmacists themselves on the initial open evening). Pharmacists themselves were the determiners of when they were sufficiently trained and therefore comfortable to give the injection to patients, in collaboration with the research team. Some pharmacists already had anaphylaxis training due to ongoing administration of influenza vaccines as part of their regular daily workload. The remaining pharmacists attended a session in how to respond to anaphylaxis given by a member of NHS Lothian's emergency care training team.

\section{Pharmacist payment}

Upon completion of each injection, pharmacists completed paperwork identifying the patient and when the injection was given. This was sent to the research team and a payment of $£ 20$ per injection plus the cost of one Sayana Press ${ }^{\circledR}$ injection ( $\left.£ 6.90\right)$ was paid to the pharmacy for participation in the research.

\subsection{Patient population and recruitment}

The inclusion criteria for participation in this research was patients that were already using the contraceptive injection, and had been doing so for at least six months, and so were receiving their third injection or more at the time of recruitment. This decision was made due to the high attrition rate of users of the contraceptive injection (Moreau et al., 2007). Women who discontinue a contraceptive method after a short period of time are likely to do so within the first two months of use (Rosenberg and Waugh, 1998). Patients who had been using the contraceptive injection for a minimum of six months would therefore be more likely to continue it as their chosen method, and less likely to leave the project due to changing methods. The patient group direction also only applied to patients who had reached menarche, were not breastfeeding or $<6$ weeks postpartum and who were between 16 and 45 years of age. Women not meeting these criteria were therefore not eligible for inclusion, as pharmacists would not have been able to administer their contraceptive injection. 


\subsection{Data gathered}

Each patient was followed up by a phone call or text message after they were due at the pharmacy for each injection. Data was collected about which pharmacy they had attended, whether they had encountered any problems, and whether they were happy to attend a pharmacy for their next injection, or whether they would like to attend the clinic and see a doctor or nurse. If they preferred this then I would see them for their next injection in order to answer any questions they had. If patients discontinued the method they were briefly asked why, and what alternative method they were using instead.

If participants encountered any difficulties getting an appointment with a pharmacist they had the contact details of the research team to phone for an appointment, to ensure no participant was put at risk of missing their injection. Any participant who chose to withdraw from the research was seen for their initial injection (or first appointment for alternative contraception) by the research team. If participants continued with the research until the end, after a year of injections (initial injection at clinic followed by 3 injections at the pharmacy) participants were invited to attend the clinic for their fifth injection and to complete a questionnaire about their experience. This was also offered to any patients withdrawing from the project who had had at least one injection at a community pharmacy. The questionnaire asked about the disadvantages and advantages of attending the community pharmacy, and also contained free text boxes wherein participants were encouraged to write their feelings about the project.

Each pharmacist involved in the project was interviewed individually using a semistructured interview format, between October 2014 and May 2015. A topic guide outlined the key areas to be covered in the interview. Interviews usually took place in the clinical room for patients, or in the pharmacy staff room. With the written consent of participants, these interviews were audio recorded and transcribed. New ideas that arose in interviews were explored in subsequent interviews. Data analysis was conducted as interviews progressed, and then afterwards. 
Results based on these methods of data collection are outlined in the following chapter. 


\section{Chapter 5 Delivering injectable contraception at the community pharmacy: results and discussion}

(A modified version of chapters 4 and 5 has been published by the European Journal of Contraception and Reproductive Health Care, under the title 'The feasibility of contraceptive injections at the community pharmacy', August 2017.)

\subsection{Introduction}

Literature presented in chapter 2 demonstrates that both patients and pharmacists are generally open to the idea of patients receiving their contraception from the pharmacy. However, there is limited evidence available that this would improve on current services, and no evidence that increased contraceptive services from pharmacies reduce unplanned pregnancy rates. A small number of pilot projects from the United States (Picardo and Ferreri, 2010; Monastersky and Landau, 2006) and Sub-Saharan Africa (Ajuwon et al., 2013; Akol et al., 2014; Stanback et al., 2014) suggest that the pharmacy may be a particularly suitable place for patients to receive their contraceptive injection. Chapter 3 detailed research to assess the feasibility of piloting this service in Edinburgh, indicating that patients were open to this idea (Heller and Cameron, 2016). Chapter 4 explained the methodology of the research, and in chapter 5 I present and discuss the results.

\subsection{Results: demographics of participants}

78 women were approached for study participation during the recruitment period: 1st October 2014 to 15th March 2016. 50 were recruited; the main reason for declining participation was that patients preferred to continue receiving their injection at the sexual health clinic they had previously attended. Those recruited had an age range of 17-48 and a median age of 28. The BMI range was 17.9-45.7, with a median BMI of 26. Women belonged to all socioeconomic groups; see table 5-1 for Scottish Maternal Index of Deprivation scores (The Scottish Government, 2012a). Most participants were nulliparous; table 5-1 also demonstrates the parity of participants. 
Table 5-1 Demographics of participants

\begin{tabular}{|l|l|}
\hline Age median (range) & $28(17-48)$ years \\
\hline $\begin{array}{l}\text { BMI (body mass index): } \\
\text { median (range) }\end{array}$ & $\begin{array}{l}26(17.9-45.7) \\
\mathrm{kg} / \mathrm{m} 2\end{array}$ \\
\hline Parity & $37(74 \%)$ \\
\hline 0 & $4(8 \%)$ \\
\hline 1 & $7(14 \%)$ \\
\hline 2 & $1(2 \%)$ \\
\hline 3 & $1(2 \%)$ \\
\hline 4 & $40(80 \%)$ \\
\hline Previous termination of pregnancy (TOP) \\
\hline 0 & $10(20 \%)$ \\
\hline 1 & $11(22 \%)$ \\
\hline SIMD (Scottish index of maternal deprivation) (The Scottish \\
Government, 2012a) & $10(20 \%)$ \\
\hline SMID 5 (affluent) & $11(22 \%)$ \\
\hline 4 & $15(30 \%)$ \\
\hline 3 & $3(6 \%)$ \\
\hline 2 & $7(14 \%)$ \\
\hline 1 (deprived) & $9(18 \%)$ \\
\hline Months using contraceptive injection \\
\hline 6 & $13(26 \%)$ \\
\hline $7-12$ & $9(18 \%)$ \\
\hline $13-24$ & $4(8 \%)$ \\
\hline $25-36$ & $11(22 \%)$ \\
\hline $37-48$ & \\
\hline$>48$ &
\end{tabular}

\subsection{Results: injections received at the pharmacy}

Of 50 patients who completed the project or withdrew (including three who were subsequently lost to follow up), 24 received at least one injection at a community pharmacy. Seven received one injection, ten received two injections and seven received three injections. 26 of the 50 participants (52\%) did not receive any injections at the pharmacy, 22 withdrew before the research was completed, and four continued the project to the end in the intention of receiving an injection at the pharmacy but were not able to do so, and received all their injections at a clinic. 48 injections were delivered in a pharmacy out of a possible 150 (32\%). Figure 5-1 demonstrates where participants received their injections. 
Figure 5-1 Flowchart of outcomes of participants

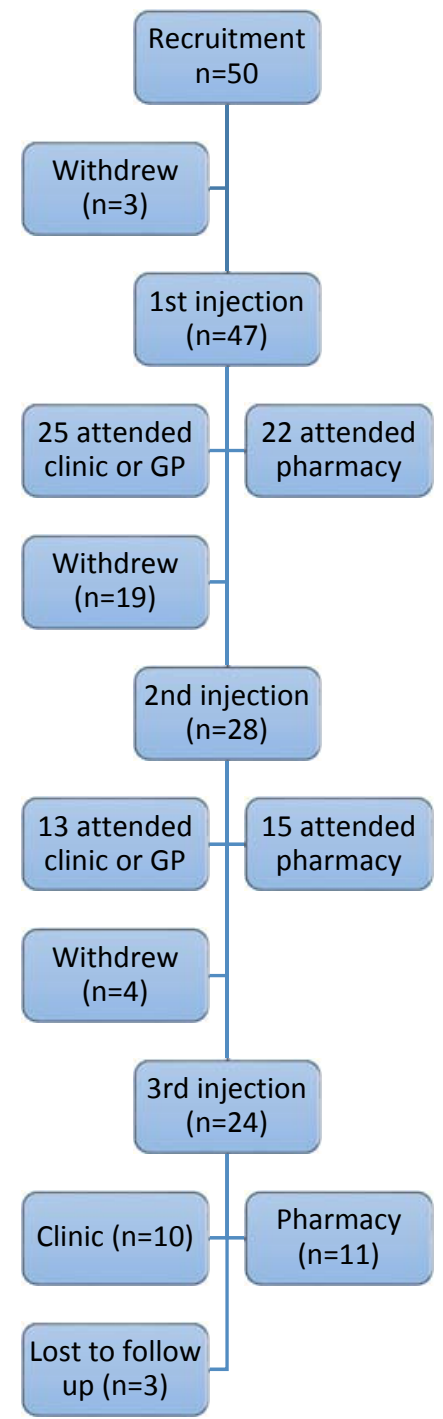

As figure 5-1 demonstrates, the withdrawal rate was high, with 27 participants (54\%) choosing not to continue with the study until its end. Three were lost to follow up after having received their second injection. Some reasons for the high withdrawal rate will be discussed further, but the reasons participants themselves gave are summarised in table 5-2. 
Table 5-2 Timings and reasons for withdrawals

\begin{tabular}{|c|c|c|}
\hline \multicolumn{3}{|c|}{ Withdrew before first injection at pharmacy due $(n=3)$} \\
\hline \multicolumn{2}{|l|}{ Left area } & 1 \\
\hline \multicolumn{2}{|l|}{ Changed contraceptive method } & 1 \\
\hline \multicolumn{2}{|l|}{ Unacceptable side effects } & 1 \\
\hline \multicolumn{3}{|c|}{ Withdrew before second injection at pharmacy due $(n=19)$} \\
\hline & $\begin{array}{l}1 \text { st injection } \\
\text { received at } \\
\text { pharmacy }(n=4)\end{array}$ & $\begin{array}{l}1 \text { st injection } \\
\text { received at } \\
\text { clinic }(n=15)\end{array}$ \\
\hline Left area & 0 & 0 \\
\hline Prefer to attend clinic & 1 & 6 \\
\hline Discontinued contraceptive injection & 1 & 2 \\
\hline Outwith protocol due to age & 0 & 2 \\
\hline Unacceptable side effects & 0 & 4 \\
\hline Prefer self injection & 2 & 1 \\
\hline \multicolumn{3}{|c|}{ Withdrew before third injection at pharmacy due $(n=4)$} \\
\hline & $\begin{array}{l}2 \text { nd injection } \\
\text { received at } \\
\text { pharmacy }(n=1)\end{array}$ & $\begin{array}{l}2 \text { nd injection } \\
\text { received at } \\
\text { clinic }(n=3)\end{array}$ \\
\hline Prefer to attend clinic & 0 & 1 \\
\hline Discontinued contraceptive injection & 1 & 1 \\
\hline Unacceptable side effects & 0 & 1 \\
\hline
\end{tabular}

By the end of the study there were 21 participants remaining that had not withdrawn or been lost to follow up. Seven had received all three injections at the pharmacy, eight had received two injections at the pharmacy, two had received one, and four participants stayed in the project but did not manage to receive any injections at the community pharmacy.

\subsection{Results: participant experience}

Of 24 participants who had received at least one injection at a community pharmacy, 22 completed an exit questionnaire. This represented $44 \%$ of participants, $92 \%$ of those who had experienced the intervention. Participants were asked about the advantages of attending the community pharmacy. Results are detailed in table 5.3. 
Table 5-3 Advantages of attending the community pharmacy

What did you find to be the benefits of attending the pharmacy?

(Respondents could tick as many as they wished, $\mathrm{n}=21$ questionnaires completed)

\begin{tabular}{|l|l|}
\hline Better opening hours & 10 \\
\hline Nearer to me & 13 \\
\hline Easier to get to a pharmacy & 15 \\
\hline Flexibility of attending different pharmacists & 6 \\
\hline Shorter waiting time & 11 \\
\hline Quicker appointment & 14 \\
\hline Can do other things at the same time & 7 \\
\hline Other (write in space): & \\
\hline Not registered with a GP so very helpful & 1 \\
\hline What one thing was the best thing about attending the pharmacy? (Respondents \\
asked to select one but 3 selected more than one, n=21 questionnaires completed) \\
\hline Better opening hours & 5 \\
\hline Nearer to me & 4 \\
\hline Easier to get to a pharmacy & 5 \\
\hline Flexibility of attending different pharmacies & 2 \\
\hline Shorter waiting time & 2 \\
\hline Quicker appointment & 5 \\
\hline Could do other things at the same time & 2 \\
\hline
\end{tabular}

Table 5-3 demonstrates the variety of responses; there was no consensus among patients on the preferred aspect of attending a pharmacy, instead they highlighted different aspects that they found useful. This was also seen when participants identified the negative aspects of attending a pharmacy, a similar diversity of answers is shown in table 5-4.

Table 5-4 Disadvantages of attending the community pharmacy

What was the worst thing about attending the pharmacy?

(Respondents could tick as many as they wished, $\mathrm{n}=21$ questionnaires completed)

Wasn't able to discuss problems I had with the method

Feel more comfortable seeing a nurse or doctor

Wasn't able to discuss other medical problems

Better opening hours at doctors/sexual health clinic

Doctors/sexual health clinic is nearer to me

Easier to get to GP/sexual health clinic

Longer waiting time at the pharmacy

Longer appointment at the pharmacy

\begin{tabular}{|l|l|}
\hline 1 \\
1 \\
1 \\
1 \\
1 \\
1 \\
1 \\
\hline 1 \\
\hline
\end{tabular}




\begin{tabular}{|l|l|}
\hline Can do other things at the same time in the sexual health clinic & 1 \\
\hline No downside to attending the pharmacy & 10 \\
\hline Other: the pharmacist wasn't there every day & 1 \\
\hline Other: I thought the opening hours would be better & 1 \\
\hline Other: the injection was painful & 2 \\
\hline $\begin{array}{l}\text { If you had to select one thing that was the worst thing about attending the pharmacy } \\
\text { what would it be? (Respondents asked to select one, n=21 questionnaires completed) }\end{array}$ \\
\hline Wasn't able to discuss problems I had with the method & 1 \\
\hline Feel more comfortable seeing a nurse or doctor & 2 \\
\hline Wasn't able to discuss other medical problems & 2 \\
\hline Better opening hours at doctors/sexual health clinic & 1 \\
\hline Doctors/sexual health clinic is nearer to me & 1 \\
\hline Easier to get to GP/sexual health clinic & 1 \\
\hline Longer waiting time at the pharmacy & 1 \\
\hline Longer appointment at the pharmacy & 0 \\
\hline Can do other things at the same time in the sexual health clinic & 1 \\
\hline No downside to attending the pharmacy & 9 \\
\hline Other: the injection was painful & 1 \\
\hline
\end{tabular}

Participants also had free text boxes where they were encouraged to tell us anything more about the project that they felt we should know. Responses reflected the mixed experience participants had and perhaps the different pharmacies involved.

Some participants found the service excellent, and commented to the research team that they thought it should be available as a service generally. For a small group of participants (eight had nothing but positive things to say) the service worked exactly as we had hoped it would.

"Very easy and positive experience! Pharmacist great" (32 year old, 6 months using injection, 3 injections at pharmacy)

"I found going to the pharmacy better than the GP. The pharmacist was very friendly and flexible with times." (25 years, 6-12 months using injection, 3 injections at pharmacy)

"Loved taking part in the trial as it has been so much more convenient than visiting the nurse every 3 months, and also don't feel that I'm wasting the nurse's time by taking up an appointment." (23 years, 12-24 months using injection, 3 injections at pharmacy)

"I thought the service was fantastic. I have a job which unfortunately leaves me with very little spare time and next to no spare time during 
regular office hours so this worked perfectly... Sad that the study has come to an end!" (28 year old, >4 years injection user, 3 injections at pharmacy)

Most participants chose one pharmacy and received all their injections there, presumably because it was conveniently located for them. Only four participants moved pharmacies during their time in the research. For one this was a considerable plus of the research:

"I found it very useful being able to attend different pharmacies and much easier to book an appointment. Some pharmacists did seem a bit anxious but I guess that will improve with time and practice. I had a good experience and think it is a really great idea." (25 years, 6-12 months using injection, 3 injections at pharmacy)

It became clear to the research team as time progressed that participants' experiences were variable according to what pharmacy they chose to use. Although numbers were too small to draw any firm conclusions, participants' attending some pharmacies gave wholly positive comments, whereas others had a more unpredictable experience. This was evident in the written feedback. One participant's comments highlight the different experience at two different independent pharmacies.

"First pharmacy attended had poor knowledge of drug. Appointments made for injection but drug not ordered so had to make multiple repeat appointments. Moved to [different] pharmacy - different experience, seamless and no problems." (43 year old, > 4 years using injection, 3 injections at pharmacy)

Some participants had a more difficult experience, with problems focusing around a few themes. A high number of participants commented that when they phoned the pharmacy to make an appointment the staff answering the phone did not know about the service and were unsure how to advise them. This comment was made even by women who found the service convenient, and were generally positive about it. The difficulty was commented on in the larger, chain pharmacists, and was more marked at the beginning of the project, but continued throughout the research. Participants found this frustrating and inconvenient, and most women who commented on this did not receive all three injections at the pharmacy. 
"When you call the pharmacy not everyone knows what you are calling for." (19 year old, 6-12 months using injection, 2 injections at pharmacy)

"The pharmacy had no idea about it the first time, the person who knew about it wasn't in. A real lack of communication." (21 year old, 2-3 years using injection, 1 injection at pharmacy)

"Problems getting appointment at the pharmacy - seemed to be confusion regarding making appointments and mixed messages from staff and lead pharmacist." (33 year old, 6 months using injection, 2 injections at pharmacy)

A small number of participants who completed questionnaires also expressed concerns about the ability of the pharmacist to give the drug. They commented on the anxiety of the pharmacist, and their apparent lack of familiarity with the injection.

"The two times I attended, the pharmacist was very, very nervous... she was very doubtful of herself. She asked me where on the stomach I normally get the injection and just seemed nervous at all times, shaky hands etc." (27 year old, 1-2 years using injection, 2 injections at pharmacy)

However, participants who chose to highlight this did not find it off putting enough to stop using the service. One person said "I have had the injection many many times before, so the above didn't phase me too much, as I know what to do and what to expect already." This was a participant in whom the pharmacist attempted the injection four times at one visit. However she explicitly states that the reason she did not receive three injections at the pharmacy was not because of this.

A high number of participants also commented on the lack of convenience of attending the pharmacy. They were unable to find a time that suited them to come in where the trained pharmacist was working. This was more evident in the chain pharmacies, where one of the trained providers might only be available a few days a week. In addition this availability changed from week to week, so participants were not able to get an idea of when they could attend in advance. The independent pharmacies usually had the trained provider there for most of the working hours of the pharmacy and were therefore more flexible for attendance. After the problem of staff who were not aware of the project wrongly advising participants when they 
called, difficulties of access and opening hours were the next most commonly mentioned comments.

"Wasn't as convenient as expected due to pharmacy opening hours and having to make appointment and check that they had the depot in stock" (43 year old, 2-3 years using injection, 3 injections at pharmacy)

"I thought the opening hours would be better, as I work 9-5 and [pharmacy] is opening later, its closer to my home and easy to get to. But it turns out there was only one trained pharmacist, who was only in this pharmacy part time, and only seemed to work at the same times I work 95. So the times didn't suit me better than going anywhere else." (27 year old, 1-2 years using injection, 2 injections at pharmacy)

The variety of comments made by participants reflects their mixed experiences. Anecdotally it seemed to the research team that two pharmacies were extremely reliable, two or three were generally reliable but participants could encounter some problems, and the rest were either rarely or never used.

\subsection{Results: community pharmacy involvement}

As has been documented in Chapter 4, we ended up starting the project with 11 pharmacies. Four were independent pharmacies, three were from smaller chains, and four were from a large national chain. Unfortunately two pharmacies withdrew during the project, one after their first injection, and one before any participants attended them. One independent pharmacist went on long-term sick leave, and one pharmacist from a small chain pharmacy left the practice, and none of his colleagues wished to participate. Another two pharmacists left whilst the research was continuing, one was replaced by a colleague who wanted to participate, and the other was not. After approximately eight months a further pharmacist was recruited, from a small chain which already had one participating pharmacist. This pharmacist also left the pharmacy after giving two injections.

There was a large variety in the popularity of the pharmacists and the number of injections each pharmacy gave. This is detailed in table 5-5. 


\begin{tabular}{|c|c|c|c|c|}
\hline & $\begin{array}{l}\text { Number of } \\
\text { participants } \\
\text { seen }\end{array}$ & $\begin{array}{l}\text { Number of } \\
\text { injections } \\
\text { given }\end{array}$ & Length of participation & $\begin{array}{l}\text { Number of } \\
\text { pharmacists } \\
\text { trained }\end{array}$ \\
\hline \multicolumn{5}{|c|}{ Independent pharmacies } \\
\hline Pharmacy 1 & 6 & 9 & Throughout & 2 \\
\hline Pharmacy 2 & 2 & 4 & Throughout & 2 \\
\hline Pharmacy 3 & 0 & 0 & Throughout & 4 \\
\hline Pharmacy 4 & 0 & 0 & $\begin{array}{l}\text { Pharmacist left before } \\
\text { any injections given }\end{array}$ & 1 \\
\hline \multicolumn{5}{|l|}{ Small chains } \\
\hline Pharmacy 5 & 1 & 1 & $\begin{array}{l}\text { Pharmacist left after } \\
\text { giving one injection }\end{array}$ & 1 \\
\hline Pharmacy 6 & 0 & 0 & $\begin{array}{l}\text { One pharmacist left six } \\
\text { months in, another } \\
\text { pharmacist was trained }\end{array}$ & 2 \\
\hline Pharmacy 7 & 0 & 0 & $\begin{array}{l}\text { Pharmacist joined after } 8 \\
\text { months, left before } \\
\text { project finished }\end{array}$ & 1 \\
\hline \multicolumn{5}{|c|}{ Supermarket chain } \\
\hline Pharmacy 8 & 7 & 13 & Throughout & 1 \\
\hline \multicolumn{5}{|l|}{ Large chain } \\
\hline Pharmacy 9 & 2 & 3 & Throughout & 1 \\
\hline Pharmacy 10 & 8 & 14 & Throughout & 2 \\
\hline Pharmacy 11 & 1 & 2 & $\begin{array}{l}\text { Pharmacist left after } 1 \\
\text { year }\end{array}$ & 1 \\
\hline Pharmacy 12 & 2 & 2 & Throughout & $1 *$ \\
\hline
\end{tabular}

\subsection{Results: pharmacist interviews}

In order to get an idea of the pharmacist experience, each pharmacist was invited to participate in a recorded, one-to-one semi-structured interview. Interviews were conducted with pharmacists from all of the above pharmacies except for pharmacies 4 and 5, where the trained pharmacists left early in the project. One was not able to be contacted and the other declined to be interviewed. Interviews were conducted with two pharmacists from Pharmacy 1, three pharmacists from Pharmacy 3, two pharmacists from Pharmacy 11 and one pharmacist from all the others. The pharmacist interviewed from Pharmacy 6 was the replacement for the pharmacist who left the project, who declined to be trained. Interviews took place after the 
project had been running for approximately 18 months. The number of injections pharmacists had given prior to interviewing ranged from zero to five. Pharmacists ranged in experience from just over three years to over 30 years.

\subsubsection{Pharmacies as a way to improve access}

All pharmacists felt that providing contraceptive services in the pharmacy was a way to improve access to them. They felt strongly that the pharmacy was easy for participants to get to and conveniently located for people. This was for a variety of reasons; almost all interviewees mentioned increased opening hours, the number of pharmacists available and the quicker appointments. They compared this to general practitioners and sexual health clinics, which they felt would be harder to get to, due to their central location and the difficulty of getting appointments. The flexibility of the pharmacy was also mentioned as an advantage. Pharmacists saw their service as convenient for women to attend and providing fewer barriers to attending than settings where other healthcare practitioners are based.

A number of pharmacists also talked about the unique relationship that pharmacists had with people attending in deprived communities. There was a perception that there was a greater familiarity than a GP would have.

"In community pharmacy, some people are in here every day. So they know me better than they know their GP. So they might feel confident to come here as well." [Pharmacist from chain pharmacy, no injections given]

A number mentioned dispensing EHC, and the idea that people were comfortable attending for this, and pharmacists were happy dispensing it. They felt that this was a useful service. Pharmacists felt that they were well placed to give advice to women, and that the pharmacist was an appropriate place for women to go for contraceptive advice. There was a perception among some pharmacists that people came to the pharmacy before going to other services, a pharmacy was a "first port of call", and so it was particularly appropriate if pharmacists could offer more services.

Pharmacists also saw themselves as contributing to the healthcare of the population in a way that "freed up" other services such as doctors. There was a view that 
enabling contraception from the pharmacy would mean that GPs had greater availability "to actually deal with emergencies". Pharmacists appointments were seen as more appropriate for routine contraception than a GP appointment, so providing this service would help the general healthcare system, which almost all pharmacists perceived as being under severe pressure. Another advantage mentioned by one pharmacist was that people might not be registered with a GP, and attending the pharmacist meant they could still access contraception. This pharmacist discussed how full the GP practices around his pharmacy were, and that he knew his customers were having difficulty registering when they moved into the area. This particular pharmacist had close links with the sexual health centre and used it to refer people he saw: "we've got your cards here, we always give them that option as well you know".

Most pharmacists did not perceive the movement of pharmacists and the covering of holidays to be a problem. They felt that this would be easily manageable in most cases and would not prove difficult for participants in the research. Although they did acknowledge that if they weren't available for a prolonged period "you're going to have to find somewhere else so it's not going to work that way", generally, even when questioned specifically, they felt that neither movement of pharmacists or pharmacist absence would interrupt service delivery unduly.

\subsubsection{Thoughts about expanded roles}

Generally pharmacists showed an enthusiastic willingness to expand their role. About half of pharmacists interviewed didn't see any downside to taking on the ability to administer the contraceptive injection. They saw it as an interesting addition to their current skill set, and a refreshing change from dispensing medication. Some pharmacists however made the point that the expanded pharmacist role was not necessarily optional, and that there was pressure from managers to constantly develop new skills, perhaps beyond what they had time to do, in order that the pharmacy could offer an increasing amount of services. "We don't have a choice really." This was mentioned by pharmacists working in chains only, the independent 
pharmacists appeared to feel in control of the decision to participate, and had done so because they felt it was a good fit for their pharmacy.

There was a perception among chain pharmacists that expansion of roles needed to be matched by sufficient time and staffing to enable this.

I think it's great, but I just don't think that we're being given enough time to actively, and proactively take part in these roles. And being given the support. [Chain pharmacist, no injections given]

Most chain pharmacists mentioned that they would be happy to continue delivering contraceptive injections, but that it was much easier to offer the service when there was a second pharmacist in the branch, and no additional cover had been made available to assist with research participation. There was a general consensus among chain pharmacists, particularly when offering the contraceptive injection long term was being discussed, that more support would need to be offered at a managerial level in the form of additional pharmacy cover, in order to enable reliable service delivery.

\subsubsection{Feelings about contraceptive injections}

A factor that the research team had not anticipated was the level of anxiety pharmacists felt about delivering the contraceptive injection. This was in part due to the fact that it was an injection and meant "breaking the skin"; a number of pharmacists talked about the anxiety this caused them. However anxiety was also attributed to the fact that they were giving contraception, a field in which the stakes were very high.

"if I don't do it well, and she gets pregnant... that would be soooo... [shocked face] ...so that was my major scare, yeah" [chain pharmacist, 13 injections given]

A number of pharmacists that were trained to give flu injections compared their anxiety to first learning to deliver that service. All found giving the contraceptive injection more stressful than the flu injection, although a number stated that this was probably due to unfamiliarity. The interviews were conducted in the early months of the research when most participants had given only a few injections. 
Many pharmacists mentioned the importance of contraception to women and that they felt therefore that it was extremely important to get it right. For some pharmacists the fact that the research was about contraception had been part of the appeal. Through their delivery of EHC a number of pharmacists were aware of the number of women not using regular contraception and therefore felt like they wanted to contribute to this unmet need through offering the contraceptive injection.

“It's frustrating as well when people come, and they've forgotten their pill or whatever, and we can't supply it... and I just think there's so much more we could do." [chain pharmacist, 3 injections given]

\subsection{Discussion}

The number of participants who received all three injections at the community pharmacy ended up being just over a tenth. Approximately a third of injections for participants were delivered in the community pharmacy setting. This was considerably less than we had anticipated. The number of withdrawals was also extremely high, with over half of the participants withdrawing before the end of the research, the majority after the first injection. Although numbers are too small to draw real conclusions, most withdrawals were from participants who had received their first injection at a clinic rather than a pharmacy. The high number of withdrawals does not therefore seem to indicate a negative pharmacy experience. However, some attempted to receive an injection at the pharmacy and were not successful, and ended up attending a clinic. This could be for a variety of reasons, as will be discussed, but as the comments in 5.4 highlight, participants had problems accessing the pharmacy service, citing lack of information among the pharmacy staff about when and how it was offered. If participants met barriers attending the pharmacy they seemed more likely to withdraw from the research and carry on attending the clinic as they were used to doing. The most common reason for withdrawing from the research given was that participants preferred to attend a clinic. Although there is little literature on this subject, when attendees of a sexual health clinic in England were offered a choice concerning various aspects of obtaining EHC, they demonstrated a preference for a doctor or a nurse (Seston et al., 2007). The pharmacists themselves did not see pharmacy access being a problem and 
many highlighted the ease of access to a pharmacy as making things more straightforward. This was not always borne out by either our numerical data or participants' comments.

Six participants also discontinued the research because they did not like the side effects experienced on DMPA-SC. This was usually a lump at the injection site, although two women who had been amenorrhoeic on DMPA-IM found they had some bleeding on DMPA-SC and discontinued for this reason. Unacceptable side effects is a reason women discontinue all contraception, including the contraceptive injection (Moreau et al., 2007). Our own research in Chapter 3 demonstrated that the main reasons participants discontinued the contraceptive injection were irregular bleeding, weight gain and acne (Heller and Cameron, 2016). However the burden that side effects cause may be weighed by women against the inconvenience of attending clinic, and so it is possible that a more straightforward experience might have led to participants continuing with research, despite some ongoing side effects.

The participants who received all three injections at the pharmacy tended to be those who were recruited later in the project. All except one were participant number 35 or higher. This may reflect the time that it took to establish the service in the pharmacies, and for the staff to become comfortable delivering it. Various factors make successful change more likely in health services. Grol et al divided theories into aspects relating to individual, aspects relating to social context and aspects relating to organisation and economic context (Grol and Wensing, 2004). In the scope of an exploratory pilot project we had restricted abilities to affect aspects like the existing pharmacy culture, and only limited economic incentives to offer. As time progressed and more participants attended successfully for their injections, the attitudinal contexts probably did shift towards a service change, which may be why the project was more successful in later stages, with more injections delivered in pharmacy and lower withdrawals. However the high number of withdrawals and the number of people who did not successfully receive their injection at the pharmacy was due at least in part to specific challenges encountered by this research. These challenges are explored here. 


\subsection{Discussion: challenges encountered}

\subsubsection{Recruitment}

The first difficulty was recruitment: this was largely a consequence of the recruitment site. Recruitment initially took place at a large urban sexual health centre. However following the conception of the research there was a concerted effort by the centre's lead clinicians to move routine contraception appointments, such as the contraceptive injection, away from specialist sexual health centres and into primary care. A triage system redirected these patients, giving priority instead to patients with symptoms of sexually transmitted infections, and those requiring emergency contraception or long-acting reversible contraception. This heavily reduced participants available for recruitment. A number of steps were taken to try and overcome this problem. The first step was to inform reception staff taking phone calls from patients that I would see any patient requesting a contraceptive injection. This strategy had reasonable success, and most participants were recruited through this pathway.

Recruitment was initially expanded to include peripheral clinics associated with the sexual health centre; these were located in areas of higher social deprivation and operated on a booked appointment system rather than a walk-in system. However although these clinics saw a high number of appropriate patients, the recruitment rate was low in this population. Peripheral clinics were already conveniently located for patients to attend: this was the reason behind their inception. Attending their community pharmacy did therefore not offer them the advantage that it might offer those who had to travel to receive their injection. Many patients had been attending clinics for years and knew the staff well, and confided to research staff that this was a reason they wished to continue attending clinic. This strategy did not therefore increase recruitment rates.

A second attempt to increase recruitment looked at recruiting participants from primary care. By the time the research had started it was clear that some pharmacists were finding it more challenging than others, for a variety of reasons demonstrated in pharmacist interviews. In addition, some pharmacists were more popular with 
participants. Three GP centres were therefore chosen that were located near pharmacies that were successfully delivering injections. The GPs and practice managers were contacted with information about the project, and invited to participate. Two practices agreed. I attended each practice to explain to the medical team about the research. Bright attractive posters and leaflets were designed, approved by the ethics committee that had originally reviewed the project, and located in the patient waiting room and nursing rooms. These encouraged patients to get in contact with the research team if they were interested in participating. Practice nurses were also encouraged to speak to patients that they saw for contraceptive injections. Despite an enthusiastic response from the healthcare professionals involved, no participants were recruited via this method.

In the end the decision was made to cap recruitment for the project at 50 participants, though the original target had been 100 . By the time 50 participants were recruited, the timescale for recruitment had already been longer than anticipated. It was also clear that there were a number of problems with the research, and it was not necessary to recruit another 50 participants to demonstrate this.

\subsubsection{Unavailability of Sayana Press ${ }^{\circledR}$}

Approximately three months after recruitment of the first participant, and after recruitment of 25 participants, Sayana Press ${ }^{\circledR}\left(\right.$ Pfizer $\left.^{\circledR}\right)$, the only licensed product for delivery of the subcutaneous contraceptive injection, became globally unavailable. It was not possible to order it from anywhere and supplies to give to patients participating in the research quickly ran out. Throughout the shortage Pfizer ${ }^{\mathbb{B}}$ was unable to anticipate when supplies of the drug would become available: the shortage lasted approximately five months. As a consequence all participants already recruited were unable to attend the pharmacy for one injection, and some were unable to attend for two injections. Instead they attended the clinic and were seen by the research team, and given the DMPA-IM that they had previously received.

During this time some good will appeared to be lost with participants. Many that had anticipated attending the community pharmacy but instead attended their regular 
clinic withdrew from the project. During the time that Sayana Press ${ }^{\circledR}$ was unavailable 17 participants withdrew from the project.

We cannot say with certainty that the reason that these 17 participants withdrew was the unavailability of Sayana Press ${ }^{\circledR}$. The questionnaire asking about reasons for withdrawal was designed before the study commenced, and the option of withdrawing due to unavailability of the contraceptive was not included. The majority of patients who withdrew during this time period cited preferring to attend clinic as the reason for their withdrawal. They declined the option of being notified when Sayana Press ${ }^{\circledR}$ was back in stock so that they could continue to receive injections at the pharmacy at a later date. Other participants received an injection from the clinic during the period of unavailability and then subsequently returned to the pharmacy. However it seems likely that the unavailability made the experience less attractive to participants, and it certainly made it less straightforward.

\subsubsection{Licensing of Sayana Press ${ }^{\circledR}$ for self-injection}

In September 2015 Sayana Press ${ }^{\circledR}$ was licensed in the UK for self-injection (Faculty of Sexual and Reproductive Healthcare, 2015a). The main sexual health centre from where recruitment took place began offering this service in July of 2016. This had a small impact on our study, as three participants left the study preferring to learn selfinjection rather than continue attending the pharmacy. As evidence outlined in chapter 2 suggests, the main perceived advantages in attending the pharmacy are access and convenience of attending. It is therefore unsurprising that self-injection at a time and place of their own choosing offers patients another level of convenience that they are keen to take advantage of. The available literature on self-injection highlights the convenience of this method (Kim et al., 2017; Beasley et al., 2014; Cameron et al., 2012b).

\subsubsection{Working with community pharmacies}

Working with community pharmacies was challenging in ways that were not anticipated when the project started. Some pharmacists working for commercial chains took months to be able to deliver the contraceptive injection. This was not the case for any independent pharmacists. Pharmacies did not have protocols for practice 
in the context of research, and all pharmacists except one were new to clinical research. Each commercial chain had forms that needed to be signed by differing levels of management before the pharmacists could deliver injections, and senior staff were unsure which of their own protocols applied to pharmacists involved in research. In addition pharmacists have a number of pressures that inevitably impacted the time available for them to spend on this research, particularly as they were not offered any extra time or financial compensation. The time taken for each pharmacist to receive full approval from each level of management delayed progress and consequently a number of participants that anticipated receiving their injection at a chain pharmacy needed to attend a clinic instead. This break in anticipated service delivery may be another explanation for the high withdrawal rate seen.

The number of people working in each pharmacy meant that the full staff was not always aware that the service was being offered. Participants attempting to make their appointment were informed on more than one occasion that this service was not available. This misinformation generated some frustration, as demonstrated in the participant comments. Attempts to rectify these communication difficulties were made by repeated site visits and contact with the pharmacy, but a high degree of staff turnover meant that many staff remained unaware of their pharmacy's participation. When asked, pharmacists involved felt confident that all staff in the pharmacy were aware of the service, and declined further visits or meetings, but this picture did not match patient's comments. Dissemination of information is a challenge in large pharmacy chains and would be an important aspect of the feasibility of the research being expanded or repeated. It is likely that the uncertainty was a consequence of this being a small research project rather than a service offered generally, but our experience demonstrates the difficulty of bringing about service change, particularly within large services employing many staff members. Grol and Wensing highlight the importance of the social context in which any change is planned. The existing culture, the opinion of key people and the observed behaviour of role models all contribute to change being more or less likely to happen (Grol and Wensing, 2004). Although we did not collect any evidence from other groups, it does not seem as if 
other staff at the pharmacy felt part of the service delivery, and were not invested in its successful implementation.

A further unexpected challenge was the number of times that pharmacists moved between pharmacies. Pharmacists who had been trained to give the subcutaneous injection could at any time be sent to work in a different pharmacy not listed as participating. Pharmacists had no control over this and found out only a week in advance. Participants had been instructed (on the pharmacists' advice) to phone up the week before their injection was due. However the pharmacist might only be working at the relevant pharmacy one or two days a week. As one key advantage potentially offered by the pharmacy was better opening hours, this was a considerable disadvantage. Participants who contacted pharmacies but were unable to arrange appointments at a time when a pharmacist was available often contacted the research team to ask for a clinic appointment, as they were anxious about putting themselves at risk of unplanned pregnancy. The pharmacists themselves did not see this as problematic. Even when explicitly asked, they felt that moving between pharmacies would be a surmountable problem that would not impact on the research. This discrepancy between the access anticipated by the pharmacist and the participant experience was conspicuous. Chapter 2's literature review demonstrated that, although there is often marked enthusiasm by pharmacists about role expansion, there is limited evidence that sexual and reproductive healthcare services will be delivered as comprehensively in pharmacies as in traditional healthcare settings. This differential was clearly evident in this aspect of our research.

Some of the challenges of working with community pharmacists on research projects have been explored elsewhere. Pharmacists often receive minimal or no training on conducting healthcare research, and they have commercial pressures in addition to those of service delivery, not found elsewhere in the NHS (Black et al., 2009). The pressures experienced by pharmacists were particularly apparent in comments made by pharmacists working within a chain. Only these pharmacists raised concerns about managerial support and cover if the service was to expand. Working within a pharmacy corporate structure carries further challenges, and can often mean rapid 
turnover in staff involved, necessitating adjustments and changes on the research team's part (Weinberger et al., 2002). A high staff turnover meant that our original plan - to train a number of pharmacists at the beginning of the research - was not the best approach, leaving us with fewer trained pharmacists than we had originally planned. In addition the comments from patients and pharmacists indicating their anxiety suggests that further training would have been useful for those that remained in the research. Although we emphasised that the pharmacists could contact the team if they wanted further training, this option was only taken up by one pharmacist, who received additional supervision at her pharmacy. The anxiety displayed is particularly relevant in the context of proposed expansion of healthcare services to community pharmacies, and the potential resources this may require. Suggestions for alternative approaches to training pharmacists in order to increase their confidence are discussed below. It should however be noted that, although some participants commented on pharmacist anxiety, none offered this as a reason for withdrawal from the research.

The response of participants and pharmacists, particularly in chain pharmacies, suggests that any contraceptive service offered would have a greater chance of success if the majority of pharmacists in most pharmacies offered the service. The confusion of some staff and the sporadic nature of the injections given seem to have had a significant influence on the experience of both participants and pharmacists. In particular the lack of awareness about the project by pharmacy staff influenced women's access, and increased access was the main hope for initiating the research. If offering the contraceptive injection at the pharmacy became routine then it is likely awareness would spread and both pharmacists and pharmacy staff would become more comfortable with the concept.

The influenza vaccination is a good example of an injection delivered successfully by UK pharmacies, both as part of the NHS and privately (Orrell et al., 2015; Brown et al., 2012). Some patients will even pay for a vaccination at the pharmacy that they would be eligible to receive at no cost (Anderson and Thornley, 2014). Evidence indicates that pharmacy vaccination results in vaccination for those who would not 
otherwise receive it; this is potentially due to better accessibility and convenience. The Isle of Wight introduced a program in 2010-2011 expanding influenza vaccinations to include community pharmacies. 1597/2207 (72.3\%) of patients who received the vaccination at a pharmacy answered a satisfaction questionnaire, and $98 \%(n=1540)$ indicated that they would use the pharmacy service again. $6.8 \%$ $(n=207)$ indicated that they would not have been vaccinated if not for the pharmacy service (Warner et al., 2013). Patient questionnaires completed by 898 patients in Grampian, Scotland, as part of a pharmacy influenza vaccination scheme in 2002, demonstrated similar views, with $37 \%(n=335)$ stating that they would not have received an influenza vaccine if it had not been offered at the pharmacy (Hind and Downie, 2006). Investigation of pharmacy vaccination in Wales in 2012/13 showed that $30.8 \%$ of patients vaccinated at pharmacy $(350 / 1136)$ had not been vaccinated the previous year. When questioned $26.7 \%$ of under- $65 \mathrm{~s}$ indicated that they would not have been vaccinated if they had not been at the pharmacy that day (Evans et al., 2013). By contrast however a 2015 survey of 7154 pharmacy customers, $63 \%$ $(n=4480)$ of whom were eligible to receive an NHS vaccine, noted that $51 \%$ $(\mathrm{n}=3620)$ preferred to receive vaccinations at their GP surgery, with $12 \%(\mathrm{n}=834)$ preferring the pharmacy (Tan et al., 2015).

Pharmacists are usually paid a small amount for delivering influenza vaccinations: in Scotland the pharmacy is reimbursed for the cost of the vaccination by the Scottish Government (The Scottish Government: Pharmacy \& Medicines Division, 2013) and private patients pay a fee of around $£ 10$ (Boots.com, 2016). Although pharmacists were paid a fee of $£ 20$ in our research this was not sufficient to remove barriers to smooth service delivery. If the service were offered routinely this fee would be unlikely to be sustainable. A key difference between our pilot project and influenza vaccination is that high-level management within pharmacies instigated influenza vaccination, and a number of systems were concurrently put in place to ensure that vaccinations could be delivered to customers. The barriers that participants discussed tended to be service delivery barriers, and minimising these might make future research more successful, as I will go on to discuss. 


\subsection{Suggestions for future research}

The small-scale nature of this research resulted in specific challenges around service delivery that may well be present in future pilot studies. However it would not be either good practice or cost effective to instigate large changes in service delivery on equivocal evidence such as we produced. Repeating the project but addressing some of the training and communication needs identified by pharmacists and staff working in pharmacy might generate more positive findings. The comments about "shaky hands" and multiple attempts being needed to give injections suggests that the training was not adequate for pharmacist confidence, even if it was deemed adequate for competence. A rolling programme of training for pharmacists to attend prior to giving an injection might alleviate anxiety and increase confidence. This style of training would also enable pharmacists joining the service to gain skills as others left, maintaining a larger pool of proficient pharmacists. Ideally future research would include adequate funding to enable pharmacists to attend training in their paid working hours by providing relief pharmacist cover.

In our research only one pharmacist from each pharmacy wished to develop the necessary skill. If more financial support was available, perhaps with remunerated time and more extensive training, more pharmacists might wish to be involved. Training every pharmacist working at pharmacies involved in service delivery would be an excellent approach, as this would remove the barrier of limited trained pharmacist availability that frustrated some participants. However the chain pharmacies had a large number of pharmacists who could potentially work in each pharmacy, and therefore training them all might prove challenging. A potential solution would be to make delivering the contraceptive injection a core pharmacist skill, as influenza vaccination now is (Orrell et al., 2015; Brown et al., 2012). Such a significant change in pharmacy services would need managerial input, as well as the support of local public health authorities, and this might be difficult to achieve without compelling research to demonstrate its efficacy and acceptability. Nevertheless, enthusiastic support at a managerial level would be considerably more likely to lead to new service implementation (Grol and Wensing, 2004). 
Our research demonstrates the importance of involving all staff at the pharmacy, not just the trained pharmacists delivering the injection. Training and information sessions held with all staff likely to be in contact with participants would minimise the chance of inaccurate information being conveyed. Ensuring that the majority of pharmacists in participating pharmacies are adequately trained, and that all staff members know about the service, might be achieved by conducting future studies using either just independent pharmacies, or pharmacists from one chain only. This approach would enable limited financial resources (which would be most usefully spent on staff to train pharmacists and compensating pharmacists for training time) to be targeted effectively. However, doing so would lose the advantage of greater flexibility for participants about where they attended, which some identified as a positive. Running the research again without the global unavailability of the product would also be likely to positively influence the participant experience.

Ultimately the licensing in the UK of Sayana Press ${ }^{\circledR}$ for self-administered injection will impact substantially on the utility of further exploring pharmacy administration. The main concern that patients cite when discussing receiving healthcare in the community pharmacy setting is lack of privacy, with resulting anxiety about confidentiality (Saramunee et al., 2014; Lambeth Lewisham and Southwark Health Action Zone, 2002; Anderson et al., 2004). The primary advantages are cited as convenience and speed and ease of access (Barlassina, 2015; Greene et al., 2006). Giving patients the option to self-administer in the privacy of their own homes at a time that suits them may therefore confer greater advantages than attending a pharmacy (Kim et al., 2017; Beasley et al., 2014; Cameron et al., 2012b). However some people may continue to prefer receiving their injection from a healthcare professional, and there is potential to further explore pharmacy delivery, adapting our research as outlined above.

\subsection{Summary and conclusion}

This was a pilot study to assess the feasibility and acceptability of patients receiving their contraceptive injection at the community pharmacy. Our study failed to show either feasibility or acceptability to participants, as reflected in the high withdrawal 
rate. This may be due to some particular challenges encountered throughout the research, as outlined in my discussion.

The most robust way to investigate women receiving their contraceptive injection from the pharmacy would be to conduct an RCT where participants were randomised to either current care (injections at a sexual health clinic or a GP) or injections received from a pharmacy. For greatest utility this would be powered to identify a difference not just in continuity rates, but also, and more importantly, in unplanned pregnancy rates. Normally a pilot project such as we conducted would assess feasibility issues concerned with an RCT, as well as identifying any barriers prior to conducting it. Any RCT, particularly one large enough to detect a difference in unplanned pregnancy rates, would require significant resources. Due to the change in licensing for self-injection, and the problems in feasibility that we have identified through our pilot trial, it would not be sensible to allocate resources to an RCT at this time. Further pilot studies are instead merited, using the lessons learnt from our research, that aim to overcome to some of the difficulties we encountered with training and staff. Indeed, despite the potential for delivering contraception in the community pharmacy as outlined in chapter 2 , any contraceptive service from pharmacies needs to be comprehensively piloted, ensuring that limited public resources are spent in the most useful and effective ways possible. 


\section{Chapter 6 Postpartum contraception: a missed opportunity to prevent unintended pregnancy and short inter-pregnancy intervals}

\subsection{Introduction}

Chapter 1 outlined the unmet need for contraception, and set out the case for expanding current service delivery. Doing so has the potential to increase contraceptive uptake and offer women better protection against unplanned pregnancy, particularly if use of the most effective LARC methods was increased. Chapters 2-5 examined delivery of contraception from the community pharmacy.

This would represent novel service delivery but unfortunately chapter 5 presented the difficulties our research encountered, and I concluded that delivering the contraceptive injection at the community pharmacy presents considerable access challenges for recipients and is not resource efficient.

As discussed in chapter 1, a key opportunity for contraceptive intervention currently gaining attention in the UK is the immediate postpartum period. Systematic reviews consistently show immediate postpartum contraception to be safe, acceptable and cost-effective (Lopez et al., 2015; Washington et al., 2015; Prager and McCoy, 2015; Goldthwaite and Shaw, 2015). Sexual health strategies from Scotland, England and Wales all encourage delivery of contraceptive services from maternity settings and agree that access to postnatal contraception should be a public health priority (The Scottish Government, 2011; The Scottish Government, 2015; Department of Health, 2013; Welsh Assembly Government, 2010). The development of a best practice paper by the Royal College of Obstetricians and Gynaecologists in 2015 entitled 'best practice in postpartum family planning' provided further impetus for clinicians, policy developers and public health strategists to recognise the importance of the postpartum period as an opportunity for women to plan their future pregnancies (Royal College of Obstetricians and Gynaecologists, 2015). In Scotland, the 2015 update to the Sexual Health and Blood Borne Virus Framework increased its emphasis on postpartum contraception, recommending that all NHS Boards in 
Scotland include antenatal counselling about contraception, and provide patients in maternity services with their preferred choice of contraception, or a bridging method, prior to discharge following delivery (The Scottish Government, 2015). Finally, at the beginning of 2017, the Faculty of Sexual and Reproductive Health's guidelines to contraception after pregnancy recommend that effective contraception should be initiated as soon as possible after childbirth, that maternity services should ensure all women have access to a full range of contraceptives, including LARC, and that intrauterine contraception and the contraceptive implant can be inserted immediately after vaginal or caesarean delivery (Faculty of Sexual and Reproductive Healthcare, 2017).

Until these guidelines, UK guidance about postpartum contraception has lacked specificity. The clinical guideline from NICE (the National Institute of Health and Care Excellence) about postnatal care up to eight weeks after birth says only that "methods and timing of resumption of contraception should be discussed within the first week of birth", and that healthcare professionals should provide proactive assistance to women who may have difficulty accessing contraceptive care (National Institute for Health and Care Excellence (NICE), 2006). There is no explanation of who may have difficulty accessing care, or what such proactive assistance might entail, and no attempt to standardise discussion. As of January 2017 this guideline is currently being updated (National Institute for Health and Care Excellence (NICE), 2017).

It is unsurprising that NICE guidance offers little clarity about what postpartum contraception counselling should comprise, as generally there is a paucity of evidence about strategies that lead to an improved uptake. Two Cochrane reviews have examined interventions; the first in 2012 investigated education about postpartum contraceptive use from RCTs, looking at 12 trials comprising 4145 women (Lopez et al., 2012). The authors of the review note the considerable heterogeneity in the contraception counselling described. Four studies provided a single counselling session about contraception to one arm of the trial, and then examined the contraception uptake at a later date. Two showed that counselling 
(structured or informal) meant the experimental group were more likely to be using contraception than the control group (Saeed et al., 2008; Torres et al., 2014). However, two other trials showed that neither a one minute script about LARC nor counselling, video and written material appeared to make a difference to the intervention group in use of the contraceptive investigated (Tang et al., 2014; Gilliam et al., 2004). It is particularly challenging to conduct RCTs in this area, as standardising the intervention is both complex to achieve and difficult to measure. The authors of the review note this difficulty, and suggest that the quality of the evidence could be improved by better reporting of the training providers received, ways of measuring adherence to the protocol and way of measuring recipients knowledge and understanding. They also note the lack of an objective outcome measure in most trials, such as a pregnancy test or structured questionnaire (Lopez et al., 2012).

An attempt is made to address some of these challenges with a 2014 Cochrane review of non-randomised studies to improve postpartum contraception use. The authors point out that educational interventions may not be suitable for RCTs, particularly as blinding is unlikely to be feasible, and funding for large RCTs may be harder to obtain. As with the RCTs discussed, most studies examined an educational intervention, where messages were given about healthy birth spacing and postpartum contraception, sexual health, lactational amenorrhoea or a combination of all three. The 2014 review examined six studies and 5143 women. Although all studies showed some association of an educational intervention with contraception use, only two showed fewer pregnancies in the treatment group. In these interventions, counselling at various times postpartum did appear to mean people receiving the intervention were more likely than comparison groups to be using a modern or effective method of contraception (Hardy et al., 1998; Lee et al., 2011). When a contraceptive method (the IUD) was actually made accessible to women, in addition to contraceptive counselling, women in the intervention group were more likely to be using it. Women in the intervention group were not more likely to be using other methods of contraception, suggesting that access to a method may make at least as much difference to women as education about it (Foreit et al., 1993). 
The Cochrane reviews demonstrate the difficulties inherent in assessing a complex and multifactorial health intervention. Engin-Üstün et al examined the effect of providing counselling to 143 women in the immediate postpartum period. They noted that after counselling, $33 \%$ of women $(n=47)$ had decided to use intra-uterine contraception. At a telephone interview at least five months later however, only $10 \%$ $(\mathrm{n}=14)$ were using this method. Although initially this might indicate the counselling was not successful, this intervention is not designed to either investigate or report barriers in place for these women getting IUC. Without this knowledge it is difficult to assess the use of the counselling as an isolated intervention, particularly as there are clear documented barriers to accessing IUC postpartum (Ogburn et al., 2005; Potter et al., 2016; Zerden et al., 2015; Harney et al., 2017).

Until the 2017 guideline there was no clear guidance about when information about postpartum contraception should be offered and when the contraception itself should be offered. Although only a very limited amount of research has been done with women themselves about what they want, what is available demonstrates only the need to tailor counselling to individual women. Available evidence shows a wide variety of opinions about what counselling should be offered and when, with women as likely to criticise providers for not providing information about the most effective methods as to feel that they were being coerced into using them (Murphy and British Pregnancy Advisory Service, 2014; Yee and Simon, 2011a; Yee and Simon, 2011b). Further research, both qualitative and quantitative, on women's feelings about discussion of contraception at various points in the antenatal and postnatal course is needed. This should be linked with birth spacing and unplanned pregnancy as objective measures, as well as the acceptability of differently timed interventions.

\subsubsection{Summary}

There is some weak evidence that educational or counselling style interventions designed to improve uptake of postpartum contraception may result in adoption of more effective methods in the postpartum period, though length of follow-up in the literature is often short term. There is weaker evidence that such interventions lead to 
fewer unplanned pregnancies. The literature provides little consensus about what postpartum women prefer.

\subsection{Justification for the methodologies}

At the time and location this research was carried out postpartum contraception consisted of a brief discussion with patients by the midwife discharging the patient. Evidence from this population indicates that contraceptive information given was basic, giving it was not a priority, and midwives felt ill equipped to deal with any discussion (McCance and Cameron, 2014). However before developing any intervention to alter services we wanted to assess whether any change was necessary. Short inter-pregnancy intervals are an independent risk factor for intrauterine growth restriction, preterm birth and neonatal death (Smith et al., 2003). A WHO Technical Consultation assessed all available evidence and determined that this risk was present in both resource-rich and resource-poor countries, and recommends a birth to pregnancy interval of two years (World Health Organisation, 2006). The first part of this research used maternity records to examine what percentage of women delivering in our maternity unit had conceived their baby within a year of a previous birth. We also examined records from women attending the termination of pregnancy service to see what percentage had conceived the pregnancy within a year of a previous birth. This evidence can be used to help assess the unmet need for contraception among this population.

As there is a lack of evidence about what women want, we also designed a selfadministered questionnaire for our postpartum population, asking about plans for future pregnancies, contraceptive counselling already received, and whether they would take up the option of immediate LARC if available to them. This was distributed on maternity wards in the immediate post-partum period.

In the context of expanding sexual healthcare beyond current service delivery models, the research described in this chapter seeks to examine both the need and the patient willingness to expand service delivery of contraception to include the postpartum period. 


\subsection{Postpartum contraception: a missed opportunity to prevent unintended pregnancy and short inter- pregnancy intervals}

Published in full: Heller R, Cameron ST, Briggs R, Forson, N, Glasier A. Postpartum contraception: a missed opportunity to prevent unintended pregnancy and short inter-pregnancy intervals. Journal of Family Planning and Reproductive Health Care 2016 42(2):93-8. Published with permission of the editor and coauthors.

\subsubsection{Introduction}

The World Health Organization (WHO) recommends that birth to pregnancy intervals be at least two years in order to optimise maternal and infant outcomes across both resource poor and resource rich countries (World Health Organisation, 2006). Data from the United Kingdom (UK) has shown that women with intervals between births of less than 12 months are at increased risk of obstetric complications, premature birth and neonatal death, even after the potentially confounding effect of maternal obstetric history has been taken into account (Smith et al., 2003). It is therefore essential that in the postpartum period there are as few barriers as possible to accessing effective contraception, particularly long-acting reversible contraceptive (LARC) methods such as intrauterine contraception and the contraceptive implant since these are the most effective at preventing pregnancy (Winner et al., 2012; Trussell, 2011).

Guidelines from the National Institute for Health and Care Excellence (NICE) state that method and timing of contraception should be discussed in the first week postpartum, although provide no guidance about the content of this discussion (National Institute for Health and Care Excellence (NICE), 2006). The Faculty of Sexual \& Reproductive Healthcare guidelines advise that time should be found in both the antenatal and postnatal period to discuss all forms of contraception (Faculty of Sexual and Reproductive Healthcare, 2009a). However, a large Internet survey of UK mothers found widespread dissatisfaction with the timing and quality of contraceptive advice received postnatally (British Pregnancy Advisory Service, 2012; McCance and Cameron, 2014). Although traditionally contraception is discussed at the six week GP visit, fertility may have returned by this time for 
women who are not exclusively breast feeding (Jackson and Glasier, 2011). There is evidence that $35-57 \%$ of mothers resume intercourse within six weeks postpartum (Connolly et al., 2005; Murphy and British Pregnancy Advisory Service, 2014) and that attendance for additional visits required to insert an intrauterine method or implant is poor at this time (Ogburn et al., 2005). Caring for a young baby, as well as fatigue and adapting to a period of change may make attendance particularly challenging. At delivery however, the mother is already in a health care setting with access to trained health professionals. Provision of implants and intrauterine contraception from the maternity service after childbirth could be convenient for women as they are already in a health care setting with skilled providers available. Although some maternity services in the UK currently provide contraceptive implants for some women before discharge home (Vanthuyne et al., 2014; Smith and McLellan, 2014), this is not universal across UK maternity services.

The Royal Infirmary of Edinburgh (RIE) is the main delivery unit for women in NHS Lothian (Edinburgh and the surrounding region), a district which had more than 9000 births in the year ending 31st March 2013 (NHS Scotland Information Services Division, 2014b). In NHS Lothian in 2013, there were 2314 induced abortions (NHS Scotland Information Services Division, 2014a). The RIE and the Chalmers Centre for Sexual and Reproductive Healthcare are the main providers of abortion in NHS Lothian. The aim of this study was to determine what proportion of women attending the abortion service had given birth in the preceding 12 months, and what proportion of women giving birth in NHS Lothian had given birth following a birth to pregnancy interval of 12 months or less. We also wished to determine views of postpartum mothers on future contraception and in particular on the theoretical acceptability of provision of intrauterine contraception and implants in the immediate postpartum period.

\subsubsection{Methods}

We examined the computerised database for women presenting for abortion at RIE and Chalmers Centre, Edinburgh between 1st September 2013 and 28th February 2014 to determine if women had given birth in the preceding year. All women 
attending the abortion service have a routine ultrasound for gestational dating, and so we calculated the inter-pregnancy interval from the date of last childbirth to the estimated date of conception of the index pregnancy (abortion request). The date of conception was assumed to be two weeks after the last menstrual period (using the ultrasound gestational age).

We used the regional maternity computer database (Trak) to identify women who gave birth during the same study period in the region. We then gathered further data on anyone with an inter-pregnancy interval of 12 months or less i.e. a previous birth within 21 months (we assumed a gestation of nine months for the index pregnancy and added 12 months for an inter-birth interval to give 21 months). This method may underestimate the number of inter-pregnancy intervals less than twelve months, as it does not take account of shorter gestations associated with preterm birth. (For reference, in 2012/13 the percentage of babies born under 37 weeks was 5.3\%, 476 out of a total of 9050 births (NHS Scotland Information Services Division, 2014b).)

Demographic data collected on women from abortion and maternity cohorts included age, parity and deprivation scores based upon postcode area of residence. Due to the different ways that the services managed data, women presenting for abortion were assigned deprivation scores (DEPCAT) based on 2001 census data about their postcode at the time of presentation (Carstairs and Morris, 1991; McLoone and Boddy, 1994), but for maternity patients the deprivation measure assigned was the Scottish Index of Multiple Deprivation (SIMD) spread into population-weighted categories of roughly equal quintiles (The Scottish Government, 2012a; NHS Scotland Information Services Division, 2010).

An anonymous self-administered survey asking women their views on postnatal contraception and contraceptive intentions was conducted between 3/10/13 and $31 / 1 / 14$ on postnatal wards and the Lothian Birth Centre (low risk delivery unit) in the RIE. Specifically, women were asked whether they would be likely to use a contraceptive implant or intrauterine contraception if these methods were available in the immediate postpartum period, before discharge from the maternity service. Questionnaires were distributed by a research midwife. Women who spoke little 
English and who were without an interpreter or who had had poor pregnancy outcomes (e.g. still birth) were not given a questionnaire. The questionnaire consisted mainly of a series of simple tick box answers or a Likert scale (very likely to very unlikely) to indicate level of agreement with a range of statements on contraception. The questionnaire also provided free space for any comments women wished to make. Women were asked to place completed questionnaires in a closed box on the ward.

The local Quality Improvement Team for obstetrics \& gynaecology approved both projects. The scientific officer of the local ethics committee reviewed the project proposal and confirmed that ethical approval was not required.

\subsubsection{Statistics}

The questionnaires were coded and the data was entered into Microsoft Excel. Statistical analysis was conducted using IBM Statistical Package for Social Sciences (SPSS) software Version 18 (SPSS Inc. Chicago, Il, USA). Descriptive statistics were performed for demographics and groups were compared by Chi squared test of significance. Statistical significance was taken as a $\mathrm{P}$ value of $<0.05$.

\subsubsection{Results}

\subsubsection{Women presenting for abortion within one year of childbirth}

Over the study period, 1179 women attended requesting abortion. Of these, 75 had given birth within the year preceding conception of this index pregnancy $(6.4 \%)$. Considering only the parous women $(n=563)$ who attended the clinic, $13.3 \%$ of women had given birth within the year preceding the index conception. The average gestation at the time of presentation for abortion was 52 days. The average number of days between childbirth and the start of the index pregnancy as determined by ultrasound was 193 (just over 6 months), with a minimum of 51 days (just over 7 weeks). The average age of women who had given birth in the previous year was 26 (17 to 37 years). $29.3 \%$ of women $(n=22)$ who attended for abortion within a year of giving birth were aged 30 or older. Table 6.1 demonstrates their demographics and compares them to the demographics of all women presenting to the same abortion 
service during this period. Women who had given birth within twelve months were more likely to be deprived $(p=0.016)$ and to be aged $20-34(p=0.021)$.

Table 6-1 Demographics of women attending for abortion who had given birth within 12 months of conception of index pregnancy compared with demographics of all women attending for abortion September 2013-February 2014

\begin{tabular}{|c|c|c|}
\hline & $\begin{array}{l}\text { Women who } \\
\text { have given } \\
\text { birth within } 1 \\
\text { year } \\
\mathrm{N}=(\%)\end{array}$ & $\begin{array}{l}\text { All women } \\
\text { attending for } \\
\text { abortion } \\
\mathrm{N}=(\%)\end{array}$ \\
\hline Total number & 75 & 1179 \\
\hline \multicolumn{3}{|c|}{ Deprivation Category* $(p=0.016)$} \\
\hline 1-2 (affluent) & $14(18.7 \%)$ & $193(16.4 \%)$ \\
\hline 3-5 (intermediate) & $44(58.7 \%)$ & $820(69.6 \%)$ \\
\hline 6-7 (most deprived) & $17(22.7 \%)$ & $146(12.6 \%)$ \\
\hline Unknown & 0 & $20(1.7 \%)$ \\
\hline \multicolumn{3}{|l|}{ Age/ yrs $(p=0.021)$} \\
\hline$<20$ & $8(10.6 \%)$ & $221(18.7 \%)$ \\
\hline $20-24$ & $24(32 \%)$ & $362(30.7 \%)$ \\
\hline $25-29$ & $21(28 \%)$ & $266(22.6 \%)$ \\
\hline $30-34$ & $17(22.7 \%)$ & $161(13.7 \%)$ \\
\hline $35-39$ & $5(6.7 \%)$ & $106(9.0 \%)$ \\
\hline 40 and over & 0 & $61(5.2 \%)$ \\
\hline Unknown & 0 & $2(0.2 \%)$ \\
\hline
\end{tabular}

\subsubsection{Women giving birth who previously delivered within one year of pregnancy}

4713 women gave birth in the region over the time period. 332 women had given birth within the preceding 21 months, (representing a birth to pregnancy interval of less than a year), representing $7.0 \%$ of the total births. When considering only the 2393 parous women, the percentage was $13.9 \%$. The average time between births of this cohort was 17 months with the shortest period being 9 and a half months between births (in this case the index birth was significantly preterm). 108 women 
( $4.5 \%$ of parous women) had a birth to birth interval of 15 months or less, representing conception within 6 months of childbirth.

The average age of the women with a birth to pregnancy interval of 12 months or less was 29 years (see table 6.2). 53.6\% $(\mathrm{n}=178)$ were aged 30 years or older. Table 6.2 demonstrates the deprivation and ages of women in both categories. There was a statistically significant difference in the characteristics of women with short birth to pregnancy interval and the general population of women giving birth. Women who had given birth within 12 months of their previous birth were more likely to come from areas of deprivation $(\mathrm{P}=0.002)$ and tended to be younger $(\mathrm{P}=0.020)$.

Table 6-2 Demographics of women giving birth who had given birth within the preceding 21 months compared with demographics of all women giving birth September 2013-February 2014

\begin{tabular}{|c|c|c|}
\hline & $\begin{array}{l}\text { Women who have } \\
\text { also given birth } \\
\text { within } 12 \text { months } \\
\mathrm{N}=(\%)\end{array}$ & $\begin{array}{l}\text { All women giving } \\
\text { birth } \\
\mathrm{N}=(\%)\end{array}$ \\
\hline & 332 & 4713 \\
\hline \multicolumn{3}{|c|}{ SIMD Quintile* $(\mathrm{p}=0.002)$} \\
\hline 4-5 (affluent) & $107(32.2 \%)$ & $1927(40.9 \%)$ \\
\hline 3 (intermediate) & $79(23.8 \%)$ & $912(19.4 \%)$ \\
\hline $1-2$ (deprived) & $137(41.3 \%)$ & $1715(36.4 \%)$ \\
\hline Unknown & $9(2.7 \%)$ & $159(3.4 \%)$ \\
\hline \multicolumn{3}{|l|}{ Age $(p=0.020)$} \\
\hline$<20$ & $10(3.0 \%)$ & $190(4.0 \%)$ \\
\hline $20-24$ & $61(18.4 \%)$ & $633(13.4 \%)$ \\
\hline $25-29$ & $83(25 \%)$ & $1165(24.7 \%)$ \\
\hline $30-34$ & $105(31.6 \%)$ & $1554(33.0 \%)$ \\
\hline $35-39$ & $64(19.3 \%)$ & $927(19.7 \%)$ \\
\hline 40 and over & $9(2.7 \%)$ & $244(5.2 \%)$ \\
\hline
\end{tabular}

\subsubsection{Questionnaires}

A total of 300 questionnaires were handed out to a total of 318 postpartum women, present in the maternity wards over the study period, representing a distribution rate of $95 \%$. The completion rate was $83 \%$ : 250 completed. The reasons why 18 women did not receive a questionnaire included inability to speak English and poor 
pregnancy outcome. These judgments were made through discussion with the midwives looking after the women on the ward. Women may have also been absent from the ward when researchers were distributing questionnaires.

Of those completing the questionnaires, $49 \%(n=121)$ had given birth before. The average age was 30 , with an age range from 16 to 47 . $20 \%$ of women were from DEPCAT categories 1-2 (affluent), 65.6\% were from categories 3-5 (moderate deprivation) and 8\% were from categories 6-7 (severely deprived). 6.4\% did not provide their postcode. Of the 242 women (97\%) who answered the question about whether or not they could recall ever having a discussion antenatally with a health care provider about future contraception, 73 women (30\%) indicated that they had discussed contraception during the pregnancy. Of this group, 56 women $(77 \%)$ had found the discussion helpful.

237 women ( $96.7 \%$ of 245 women) stated that they were not planning a baby in the next year. Table 3 shows which methods of contraception women intended using.

Only 32 women (12.8\%) were planning on using the contraceptive implant or intrauterine contraception and 27 (10.8\%) were planning male or female sterilisation. $35.2 \%$ of women (88) indicated that they did not know what contraception they would be using postnatally. $4 \%(n=10)$ women indicated that contraception was not necessary for them. The most popular method that women were planning to use was condoms (see table 6.3).

Table 6-3 Intentions for contraceptive use

\begin{tabular}{|l|l|}
\hline & $\mathrm{n}=250(* \%)$ \\
\hline Don't know & $88(35.2 \%)$ \\
\hline Condoms & $71(28.4 \%)$ \\
\hline Progestogen only pill & $28(11.2 \%)$ \\
\hline Combined oral contraceptive pill & $24(9.6 \%)$ \\
\hline Progestogen only implant & $16(6.4 \%)$ \\
\hline Intrauterine contraception** & $16(6.4 \%)$ \\
\hline Progestogen only injectable & $13(5.2 \%)$ \\
\hline
\end{tabular}




\begin{tabular}{|l|l|}
\hline Male sterilisation & $14(5.6 \%)$ \\
\hline Contraception not necessary & $10(4 \%)$ \\
\hline Female sterilisation & $10(4 \%)$ \\
\hline Lactational amenorrhoea & $7(2.8 \%)$ \\
\hline Combined contraceptive patch or ring & $2(0.8 \%)$ \\
\hline Question not answered & $5(2 \%)$ \\
\hline $\begin{array}{l}\text { * Numbers add up to more than 100\% as some respondents ticked } \\
\text { more than one answer }\end{array}$ \\
\hline $\begin{array}{l}\text { **Intrauterine contraception includes both the copper intrauterine } \\
\text { device and Levonorgestrel releasing intrauterine system }\end{array}$ \\
\hline
\end{tabular}

Of 239 women who answered the question about whether they would choose an intrauterine method if it could be inserted prior to leaving the hospital, 78 (32.6\%) described themselves as "very likely" or "quite likely" to choose this. Of 241 women answering the same question about the likelihood of choosing the contraceptive implant if it could be fitted before leaving the hospital, 74 (30.7\%) said they would be "very likely" or "quite likely" to choose this. Combining the numbers of women likely to choose either intrauterine methods or implants gives a total of 107 respondents $(42.8 \%)$ who stated they would opt for one of these methods if insertion were available before leaving hospital. The difference in planned use of implant and intrauterine method and 'theoretical' use of the method if it was immediately available was statistically significantly $(\mathrm{p}<0.0001)$. Only 12 women $(4.8 \%)$ who completed questionnaires were aged 20 or under and six (50\%) indicated that they would be very likely or quite likely to choose a contraceptive implant if available before discharge home.

\subsubsection{Discussion}

Almost 1 in 13 women in our population presenting for abortion or giving birth have conceived within a year of giving birth. Of women who already have children, over one in eight requesting an abortion or giving birth conceived again within one year of their previous birth. Yet the survey suggests that the vast majority of postnatal women do not plan on having another pregnancy within the next year. Does this suggest that we are failing to meet the contraceptive need of women postpartum? It may well do. Most women had not discussed ongoing contraception during the 
pregnancy with a health professional and most were unsure about what method they would use. Although one in 10 were considering intrauterine contraception or implants, almost one in two women indicated that they would ' in theory' choose these methods if they could be provided before they left hospital. This interesting finding may reflect the convenience of this option, or the removal of important barriers that would otherwise deter them from choosing this method. Immediate postpartum provision of these methods could therefore be an important strategy to prevent unintended pregnancies for women in the same way that immediate uptake of LARC post-abortion reduces a woman's risk of having another abortion in the next two years (Cameron et al., 2012a). There is also evidence that uptake of contraceptive implants in the early postpartum period reduces the risk of another pregnancy in young women in the next 12 months (Tocce et al., 2012b; Lewis et al., 2010). Although there has been some concern about the impact of early initiation of progestogen on breastfeeding, the available evidence is consistently reassuring and shows no adverse effects on lactation or infant growth (Cameron, 2014).

Women who attended for an abortion within a year of giving birth were more likely to be from areas of deprivation as were women who gave birth to a baby that had been conceived within one year of childbirth. Nevertheless, both women who gave birth after a short inter-pregnancy interval and those attending for an abortion were from a wide age range and all socio-economic groups, suggesting that all women, not only those from vulnerable groups (who are often the focus of targeted, contraceptive interventions) (Lewis et al., 2010; Wilson et al., 2011; Wilson et al., 2013; Lopez et al., 2012; Stevens-Simon et al., 2001) could benefit from immediate access to implants and intrauterine contraception postpartum.

Although a significant proportion of women expressed a desire for immediate intrauterine contraception, this service is not usually available in the UK, with insertion usually taking place after four weeks postpartum (Faculty of Sexual and Reproductive Healthcare, 2009b). There is however reasonable evidence from low and middle-income countries that post-placental insertion of intrauterine contraception (within 10 minutes of placental expulsion) is safe and effective (Kapp 
and Curtis, 2009; Grimes et al., 2010; Chen et al., 2009; Lara et al., 1989), although expulsion rates of between 7 and 25\% have been reported (Kapp and Curtis, 2009; Grimes et al., 2010; Chen et al., 2009; Mwalwanda and Black, 2013). Insertion immediately postpartum may also be easier since the cervix is dilated. Small studies also show that insertion at elective caesarean section may be a particularly beneficial option, with low expulsion rates of 0-4\% (Lara et al., 1989; Chi et al., 1986; Levi et al., 2012).

A major limitation of our study is that the survey asked women about what they would theoretically choose: i.e. they are open to the idea in theory, but this is not the same as evidence of acceptability. Addressing the question of whether women would choose LARC immediately post-partum and whether this would result in fewer unintended pregnancies and fewer short inter-pregnancy intervals will need further research. In addition, this study is unable to determine what proportion of the pregnancies following a short inter-pregnancy interval was intentional. Any intervention to improve uptake of LARC postpartum would ideally therefore contain an educative component which makes clear the risks of short inter-pregnancy intervals, as well as providing the means to prevent them. Finally, although our research was conducted in one of the largest maternity services and abortion services in the UK, our findings cannot be assumed to reflect others throughout the UK.

\subsubsection{Conclusions}

This study shows that short inter-pregnancy intervals are not uncommon, However our survey data suggests that a significant proportion of women might be open to choosing one of the most effective methods of contraception (intrauterine contraception or the implant) in the immediate postpartum period, if this option was available. Provision and uptake of these methods at this time could in theory prevent more unintended pregnancies that currently end in abortion, miscarriage, ectopic pregnancies or short inter-pregnancy intervals. This would have important health benefits for women, their children and their future opportunities. Research is therefore necessary to progress strategies to provide LARC in the immediate postpartum period and to evaluate the acceptability and effectiveness of such 
strategies. This will necessitate both training of healthcare providers in maternity services to counsel and provide LARC to women postpartum, and funding to provide this service.

\section{End of published paper}

\subsection{Conclusion}

The paper presented here demonstrates clearly that in our patient population there is both a need and a willingness to expand contraceptive care to include the postpartum period. Short inter-pregnancy intervals leading to both termination and birth are clearly present in this population, and span all maternal ages and socioeconomic groups, in keeping with larger data examining inter-pregnancy intervals in Scotland (Smith et al., 2003). However, in isolation this data is not sufficient evidence of an unmet need for contraception in this population. It is plausible that women either choose to space their pregnancies with full knowledge of the inherent risks, or would prefer to risk unplanned pregnancy than to receive contraception in this period.

Our survey suggests that this is not the case, particularly given the high number of women who say they are not planning a pregnancy in the next year following a birth. This evidence is in keeping with the theory that the immediate postpartum period is a time when women may be particularly motivated to avoid unplanned pregnancy (Cameron, 2014). The number of women who say that they would be likely to choose insertion of LARC immediately post-partum if it was available further emphasises this motivation. The enthusiasm for this option points clearly towards a further intervention to explore, namely routinely offering LARC in the immediate postpartum period. One option to make LARC available to postpartum women with as few barriers as possible will be explored in chapter 7 . 


\section{Chapter 7 Intrauterine contraception inserted at caesarean section}

\subsection{Introduction}

Chapter 6 introduced the need to improve contraception delivery in the immediate postpartum period. One in 13 women requesting termination had conceived the pregnancy within one year of childbirth and one in 13 women who gave birth had conceived within one year of childbirth. $32.6 \%$ of women would like an intrauterine device inserted in the immediate postpartum period (Heller et al., 2016).

Long acting reversible contraception (LARC) methods such as the copper intrauterine device (Cu-IUD) and levonorgestrel intrauterine system (LNG-IUS), and the subdermal implant are known to be the most effective methods of preventing unintended pregnancy (Winner et al., 2012; Trussell, 2011). The prevalence of use of intrauterine contraception (IUC) in the UK is 8-9\% (Cea Soriano et al., 2014a; Lader, 2009), and in 23.2 per 1000 women in Scotland (NHS Scotland Information Services Division, 2016b). Traditionally women choosing IUC postpartum may require several visits with a healthcare provider before insertion (Lunniss et al., 2016). Each visit may represent a significant barrier for women who are likely to be recovering from childbirth (particularly after a caesarean section) and caring for a new baby, and additional visits have been identified by women themselves as a reason they do not receive postpartum LARC after choosing it (Zerden et al., 2015).

Inserting IUC at the time of caesarean section is not new, with studies showing efficacy dating back to the 1970s and 1980s (Newton et al., 1977; Ruiz-velasco et al., 1982; Chi et al., 1984). However the practice is now gaining increasing attention from obstetricians and gynaecologists as a straightforward and efficient way to provide obstetric patients with immediate effective contraception (Blumenthal and Goldthwaite, 2015). In this chapter I will initially examine the evidence for the insertion of IUC at the time of caesarean section. I will then detail and appraise research conducted with our patient population in Edinburgh investigating making IUC at the time of caesarean section routinely available to all patients undergoing elective procedures. 
(A modified version of this chapter has been published in Acta Obstetricia et Gynecologica Scandinavica, under the title 'Routine provision of intrauterine contraception at elective cesarean in a national public health service: a service evaluation.' September 2017.)

\subsection{Literature review}

In order to examine the current evidence on this subject, a literature search was conducted in Medline. Search terms were intrauterine device OR intrauterine device, copper OR intrauterine device, medicated AND caesarean section (keyword) or caesarean section (exploded). The search was limited to humans and articles in English. 93 articles were retrieved. After review by title and abstract, 30 articles were found to be relevant, including 6 reviews of the literature, 4 of which were from the last five years. References of these four reviews were also examined for papers but no additional literature was found. Papers were included if they were research, or reviews of research, about the insertion of intrauterine contraception at the time of caesarean section.

Studies from the 1980s and 1990s showed that insertion at caesarean section was safe and associated with low complication rates (Liu et al., 1983; Chi et al., 1984; Chi et al., 1986; Zhou and Chi, 1991; Xu et al., 1992). These studies investigated devices used in China that were intended to be permanent, so their utility with regard to modern devices used in the United Kingdom is limited. Celen et al undertook an observational study in Turkey in 2004, inserting copper IUDs in 235 women within ten minutes of placental expulsion. $26 \%$ of these were in women who had a caesarean section; total number was not given but would have been 61-2. Expulsion rate was $12.3 \%$ by twelve months (total data), and continuation rate was $76.3 \%$ at six months, with a follow up rate of $78 \%$ (Celen et al., 2004). A much smaller observational study was undertaken in 2005 in South Africa, looking at the Mirena ${ }^{\circledR}$ LNG-IUS (levonorgestrel-releasing intra-uterine system) inserted at caesarean section in 33 women. Information was not given on expulsion or removal rates but 20 patients still had a device in situ at six months (Puzey, 2005). In 2007 Celen's team in Turkey undertook further research, examining 245 women who had copper 
IUDs inserted at caesarean section within ten minutes of placental removal. There was one case of unplanned pregnancy $(0.4 \%)$, and the cumulative expulsion rate at six months was $17.6 \%$. Continuation rates at six months were $62 \%$ (Celen et al., 2011).

A study of 1317 women who had a copper IUD inserted after delivery of the placenta after either vaginal delivery or at caesarean section was undertaken by Shukla et al in India. 653 had insertion during caesarean section. The cumulative expulsion rate was $10.1 \%$, but the paper does not differentiate between expulsion rate for vaginal and caesarean deliveries, and this is based on a follow up rate of only $11.2 \%$.

Continuation rates at six months are also not given, and it is therefore difficult to draw conclusions about long or even medium term effects from this study, despite large numbers (Shukla and Qureshi, 2012). Follow up was more successful in a pilot study undertaken with 90 patients in Chapel Hill, USA, each of whom had a copper IUD inserted at the time of caesarean section. $47 \%$ of women were successfully contacted at six months by telephone, when no expulsions or removal were reported. However $26 \%$ of women who returned for follow up and had no visible threads did not return for their ultrasound to confirm location: expulsion cannot be ruled out among this patient population (Levi et al., 2012).

Extensive follow up was carried out by Elsedeek et al on 143 women who had either a copper IUD $(n=63)$ or an LNG-IUS $(n=80)$ during caesarean section. Women were seen weekly for six weeks, monthly for a year and then every three months, an ultrasound was performed at every visit and women answered a questionnaire. No expulsions were seen over five years in the LNG-IUS group, and four in the IUD group (6.3\%). Removal rates were very low: $7.9 \%(n=5)$ in the IUD group and $3.8 \%$ $(n=3)$ in the LNG-IUS group. Total continuation rates are not given. The ultrasounds performed gives useful data that is not available from other studies. After placement during caesarean delivery, in $65 \%$ of women (using both copper IUD and LNG-IUS) the device appeared to be abnormally placed on ultrasound performed at six weeks. A copper IUD was more likely to 'self-correct', but the efficacy of an LNG-IUS device did not appear to be affected by its position within the uterus - this is to be 
expected given that its method of action does not work primarily by its mechanics, but by its hormonal effect (Faculty of Sexual and Reproductive Healthcare, 2015b). Unusually, in this study a failure rate of $6.3 \%(n=4)$ was seen in the IUD group (Elsedeek, 2015).

Dias et al performed ultrasounds on 91 postpartum patients in Sri Lanka, 31 of whom had had a post-placental IUD insertion at caesarean section. Ultrasounds were performed prior to hospital discharge and at six weeks following delivery. Measuring the distance between the lower end of the IUD and the internal os on ultrasound at discharge was found to be moderately accurate in predicting IUD retention, but expulsion rates in this study $(22.4 \%$ after vaginal delivery and $25.8 \%$ after caesarean section) were higher than rates quoted in other studies. This study also found that mode of delivery had no impact on IUD retention (Dias et al., 2015). The fact that mode of delivery did not influence the expulsion rate of the IUD was also noted by Sucak et al in Turkey. They compared expulsion rates for 51 women who underwent an elective caesarean, 47 women who underwent a caesarean during active labour, and 62 women who had a vaginal delivery. There was no significant difference in either expulsion rates at six weeks, or cumulative expulsion rates at 12 months follow up (Sucak et al., 2015). This study is of particular interest, as there is little literature examining women who have undergone a caesarean during active labour.

A further study on a subset of Elsedeek's population looks specifically at bleeding in the puerperium between 80 controls (no device inserted at caesarean), 75 women who had a copper IUD inserted at caesarean and 65 women who had an LNG-IUS inserted at caesarean. Women who had an LNG-IUS inserted had a significantly shorter and lighter puerperium compared to the other two groups (20.2 \pm 7.7 days), and women with a copper IUD had heavier menstrual periods once menses returned compared to the control group, but this difference was not significant (Elsedeek, 2012).

A case controlled study in Pakistan examined three groups of women: 50 who had a copper IUD inserted at caesarean section, 50 who had a caesarean without an IUD, and 50 who had an IUD inserted 3 months following a vaginal delivery or abortion. 
There was no significance difference in length of hospital stay or heavy lochia between the two caesarean groups, and no difference in willingness to continue with IUD at one week, six weeks or six months between the caesarean and interval insertion groups (Bhutta et al., 2011).

An RCT (randomised controlled trial) produces the highest level of evidence to compare two different options for patients. There have been five RCTs randomising women to insertion at the time of caesarean section versus insertion six to eight weeks later. In 2014 Whitaker et al in Chicago, USA randomised 42 women to receive an LNG-IUS at the time of caesarean section or four to eight weeks later. Use of the LNG-IUS was higher in the group who had it inserted during caesarean section (60\% vs. $41 \%$ ), but not significantly so. Expulsion was significantly more common in these women ( $20 \%$ vs. $0 \%)$. The number needed in each group to assess a significant difference in LNG-IUS continuation rates was calculated to be 46 , but the trial was stopped early due to lack of recruitment (Whitaker et al., 2014). Lester et al conducted a small RCT in Uganda with 68 women undergoing caesarean delivery, 34 receiving an IUD during their caesarean and 34 receiving it six weeks afterwards. The primary outcome was use of the IUD at six months post-delivery, and use in the caesarean insertion group was $79 \%$ versus $47 \%$ in the delayed insertion group, a difference that was statistically significant. Of note, only approximately $53 \%(n=18)$ of women in the delayed insertion group received an IUD (Lester et al., 2015).

As a development of their earlier pilot in 2012 (Levi et al., 2012), Levi et al conducted an RCT in New York, USA, randomising 112 women to insertion of an IUD at the time of caesarean or six or more weeks later. Follow up at six months showed continuing use of IUD by $83 \%$ of women randomised to insertion during caesarean compared to $64 \%$ of women randomised to insertion six or more weeks later, with a $95 \%$ confidence interval of $1.02-1.66$. Satisfaction was also high in both groups (92\% and 100\% respectively) (Levi et al., 2015). A small RCT in Australia examining just the LNG-IUS randomised 48 women to LNG-IUS ( $n=23)$ insertion at the time of caesarean versus six weeks postpartum $(n=23)$. This study took satisfaction rates as its primary endpoint, and noted no significant difference in 
satisfaction rates between the two groups at six weeks, three months or six months. Four women in the interval insertion group withdrew prior to receiving an LNG-IUS but, in contrast to Lester et al, the remaining $82 \%$ of women in the delayed cohort attended for a six week visit (Braniff et al., 2015). Each of the RCTs mentioned uses small numbers only, and follows women up for a relatively short amount of time six months or a year. As the WHO recommends a birth-to-pregnancy interval of two years (World Health Organisation, 2006) follow up of this length is therefore inadequate to determine whether post-caesarean insertion of IUC leads to successful birth spacing. Numbers in RCTs are also small, perhaps due to the difficulties of recruitment described in Whitaker et al. (Whitaker et al., 2014).

There is a very small amount of literature looking at modifying techniques or devices to improve patient outcomes with insertion of IUC at caesarean section. A small pilot in Los Angeles, USA looked at tying additional suture material to strings of the copper IUD in just seven women, in at attempt to ensure the threads were always visible for removal. All women involved had threads visible at the os after six weeks (Nelson et al., 2009).

A number of systematic reviews have assessed the insertion of intrauterine contraception in the immediate postpartum period, though none have focused specifically on provision at caesarean section. A Cochrane review is recognised as being one of the most rigorous assessments of literature on a medical subject. In 2015 a Cochrane review examined intrauterine devices in the postpartum period, investigating insertion after all types of delivery, using only RCTs. This was an update of a previous Cochrane review on the same subject (Grimes et al., 2010). Of trials that have a full report of data available, the ones used by the Cochrane review are the ones mentioned in this chapter ((Whitaker et al., 2014; Lester et al., 2015; Levi et al., 2015)), other than Braniff et al, which was published after the literature search for the review had concluded. The authors of the review found that evidence was limited due to small sample sizes and limited reporting - particularly in older studies. However, the conclusion was that the benefit of providing effective contraception immediately after delivery may outweigh the disadvantages, namely 
increased expulsion risk (Lopez et al., 2015). As expulsion risk appears to only be increased after a vaginal delivery, and not a caesarean section (Lopez et al., 2015; Sucak et al., 2015), this Cochrane review presents an especially compelling case for insertion of intrauterine contraception at the time of caesarean section.

The most recent evaluation, by Goldthwaite and Shaw in 2015, concludes that the use of highly effective contraception immediately postpartum protects against short inter-pregnancy intervals and unintended pregnancy. They highlight the fact that women in the delayed insertion arm of studies often fail to receive effective contraception, and are therefore ultimately less likely to use effective methods (Goldthwaite and Shaw, 2015). Similar conclusions have been reached by other systematic literature reviews (Sonalkar and Kapp, 2015; Mwalwanda and Black, 2013; Kapp and Curtis, 2009), and this body of evidence is reflected in the recent UK guidelines about contraception after pregnancy (Faculty of Sexual and Reproductive Healthcare, 2017).

\subsubsection{Summary}

There is good evidence that insertion of IUC at caesarean is safe, convenient and acceptable, and has high continuation rates. However, this data comes mainly from low-income countries and the United States. There is no data from the UK or from Europe. With a caesarean section rate of $30 \%$ in the UK in $2014 / 5$, there is the potential to offer IUC at caesarean to a significant proportion of women. We therefore decided to conduct research with our own patient population, offering insertion of IUC at caesarean section.

\subsection{Justification for the methodology}

Although there have been relatively few, the evidence from RCTs, the highest level of scientific evidence, is that women who have intrauterine contraception inserted in the immediate postpartum period, including at caesarean section, are more likely to be using it six to 12 months later. This has been the conclusion of two Cochrane reviews and multiple other reviews of the literature. We therefore decided not to instigate a randomised controlled trial in our clinical setting, particularly given documented difficulties of recruitment (Whitaker et al., 2014). Instead we chose to 
examine the practicality and feasibility of making insertion of IUC at caesarean section routine practice in a non-fee paying healthcare system. In systems where contraception is available only for a charge, availability of free devices within a research setting can alter the findings of who chooses a device and who attends for follow-up. The devices in the study by Levi et al were paid for by TEVA, a pharmaceutical company, and this may have influenced women's choice of device (Levi et al., 2015). In the United Kingdom all contraception is available free of charge.

Research conducted with our population outlined in chapter 6 demonstrates both the short inter-pregnancy intervals present and a willingness among postpartum women to consider immediate postpartum contraception (Heller et al., 2016). By routinely offering IUC at the time of caesarean section we were able to gather data on how many women took up this offer, knowing that the only determinant of their choice was timing. In addition by collecting data on routine procedure we were able to examine barriers to instigating this practice as part of a real-life healthcare setting.

NHS Lothian began a series of pilot initiatives in 2013 to improve the uptake of postpartum contraception. This involved the introduction of antenatal contraceptive counselling by midwives and provision of contraception to women living in one area of the region from the maternity service after delivery (Cameron et al., 2017). As part of this initiative this study aimed to investigate the feasibility of routinely offering all women within this region who were undergoing an elective caesarean section the opportunity to have IUC insertion at this time, and to assess uptake, complications, continuation rates and acceptability.

\subsection{Methods}

\subsubsection{Training materials}

A training package and video were developed that included the technique of insertion of IUC at caesarean section, medical eligibility criteria for contraceptive use (Faculty of Sexual and Reproductive Healthcare, 2016), contraindications to IUC postpartum, and what post-procedure counselling with women should include. We also produced checklists that obstetricians could use for counselling women about IUC, detailing 
the risks, benefits and possible side effects and written information for women about what to expect after IUC insertion and what follow-up was necessary. All of these materials were presented to consultants and obstetric trainees working at the two maternity hospitals in the region (approximately 35 doctors). Consultants underwent one individual training session and registrars observed the video as part of their ongoing training. Ongoing peer-supervision in theatre was encouraged and adopted until all staff were trained, this took approximately four months. Obstetricians were instructed that following delivery of placenta the IUC should be removed from its inserter and placed at the fundus through the uterine incision. They were instructed to use artery forceps to guide the threads of the device towards the cervix, and through the cervix if possible. Threads of the device were not cut. The uterus, sheath and skin were then sutured as per the obstetrician's normal practice. In addition, training on contraception and information about IUC insertion at caesarean was also provided to hospital midwives.

From the 22nd June 2015, information about all types of postpartum contraception was included with routine information sent to women scheduled for an elective caesarean section three to four weeks before their scheduled caesarean date. They also received a letter explaining that if they wished to use an IUC postnatally they might be able to have it inserted during their caesarean section. Women were invited to discuss this option with their obstetrician in advance of delivery. This was in addition to the routine antenatal contraceptive counselling that some women in the region at that time received with a midwife at 22 weeks (Cameron et al., 2017). The telephone numbers of the evaluation team were included so that women could contact the team if they had any unanswered questions about IUC at caesarean. When women were consented for their delivery on the day of caesarean section those who had made the prior decision to have IUC also gave written consent for insertion of IUC.

Following delivery all women who had elective caesareans were routinely reviewed by obstetricians. Both obstetric and midwifery staff provided verbal and written information about the device that was inserted and what to expect in coming weeks. 
All women who had IUC fitted were identified by the evaluation team using electronic maternity records (TRAK). These women were contacted and offered a six week IUC thread check at their local hospital or sexual health centre with the evaluation team, consisting of myself and a research nurse. At this visit women had the opportunity to ask any further questions about IUC. They underwent a speculum to look for the threads of the IUC and trim them if necessary. If no threads were seen, an ultrasound scan was conducted to ensure the device was in situ. A partial expulsion was diagnosed if the stem of the device was seen on clinical speculum examination or if an ultrasound scan revealed the device to be in the cervical canal. Women were also asked how satisfied they were with having IUC inserted at the time of caesarean. Women were then contacted by phone or text at three, six and 12 months to determine continuation rates, and satisfaction with IUC as a method of contraception. Our primary outcome measures were uptake of IUC and complications by six weeks. Secondary outcomes were continuation and satisfaction with IUC at 12 months.

In addition to the information gathered at follow-up, the regional hospital database was reviewed for each woman in order to ensure that data on any complications was accurate. For all women who were lost to follow up both the regional hospital database and the local sexual health database were examined, to gain any further information about the presence or removal of IUC, and any further pregnancies. Sample size was not determined on an a priori hypothesis. Data continues to be collected and I report here data from those women who have completed 12 months follow up.

The local Quality Improvement Teams for women's services and sexual and reproductive health approved the project. The scientific officer of the local ethics committee reviewed the project proposal and confirmed that ethical approval was not required as this was a health service evaluation project.

\subsubsection{Analysis}

All data was entered into an excel database, and only descriptive statistics were used. 


\subsection{Results}

During the time period (13th July 2015 to 2nd March 2016) 877 women were scheduled for caesarean section, and were sent information about having IUC inserted at delivery. 120 women chose this option (13.7\%). Demographics of women who chose IUC are detailed in table 7-1.

Table 7-1 Demographics of women who chose IUC

\begin{tabular}{|c|c|}
\hline Age median (range) & $33(21-41)$ years \\
\hline $\begin{array}{l}\text { BMI (body mass index) at } \\
\text { booking: median (range) }\end{array}$ & $\begin{array}{l}26.25(17.0-49.7) \\
\mathrm{kg} / \mathrm{m} 2\end{array}$ \\
\hline \multicolumn{2}{|l|}{ Parity } \\
\hline 0 & $9 \quad(7.5 \%)$ \\
\hline 1 & $80 \quad(66.6 \%)$ \\
\hline 2 & $26 \quad(21.6 \%)$ \\
\hline 3 & $2 \quad(1.7 \%)$ \\
\hline 4 & $2 \quad(1.7 \%)$ \\
\hline $4+$ & $1 \quad(0.8 \%)$ \\
\hline \multicolumn{2}{|c|}{$\begin{array}{l}\text { SIMD (Scottish index of maternal deprivation) (The } \\
\text { Scottish Government, 2012a) }\end{array}$} \\
\hline SMID 5 (affluent) & $33 \quad(27.5 \%)$ \\
\hline 4 & $22(18.3 \%)$ \\
\hline 3 & $20(16.6 \%)$ \\
\hline 2 & $\begin{array}{ll}24 & (20.0 \%)\end{array}$ \\
\hline 1 (deprived) & $21 \quad(17.5 \%)$ \\
\hline
\end{tabular}

114 devices were inserted at the time of caesarean, 100 LNG-IUS and $14 \mathrm{Cu}-\mathrm{IUD}$. Two women changed their minds and four devices could not be inserted due to postpartum haemorrhage. All of the latter four women attended the local sexual health service for subsequent insertion of IUC $(n=3)$ or insertion of a subdermal contraceptive implant. $(n=1) .103$ women attended for a six week follow up appointment, and a further nine were contacted at this time by phone. The percentage of women with information available at each interval is detailed in figure 7-1. 
Figure 7-1 Percentage of women with information available at follow up

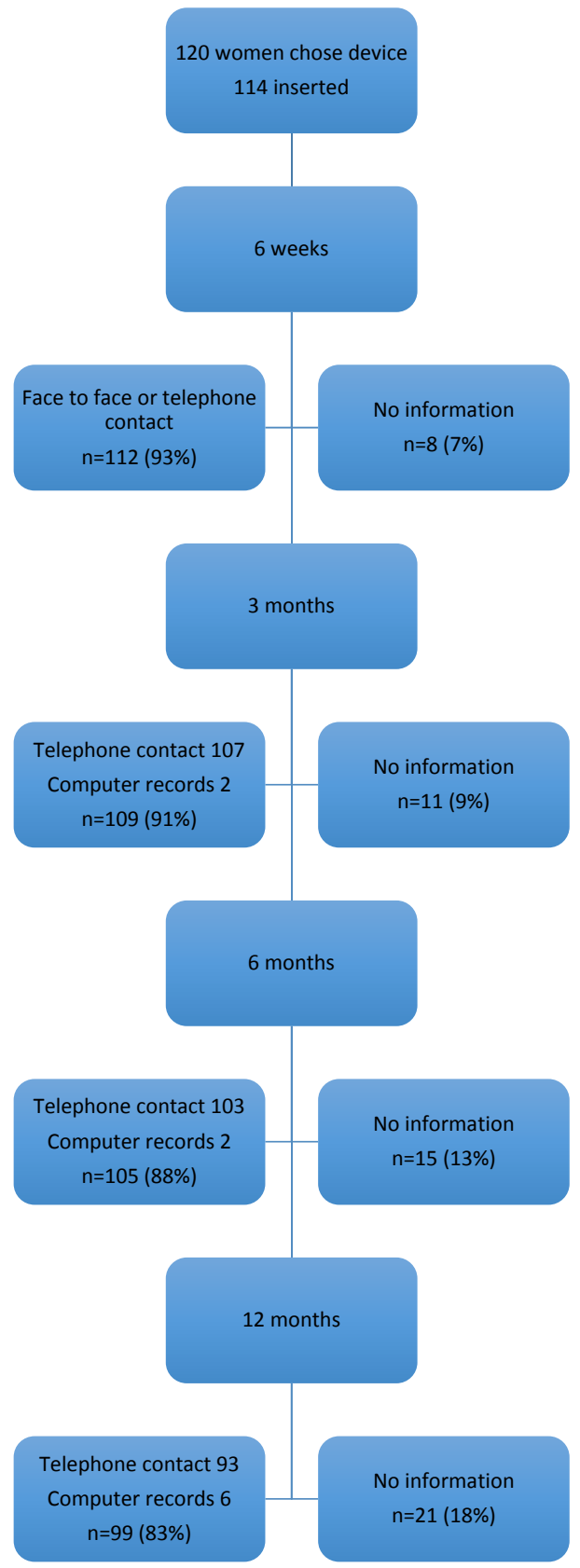

\subsubsection{Outcomes and complications}

Outcomes of IUC insertion are detailed in figure 7-2. 100/114 women who had IUC inserted at caesarean (88.5\%) attended for a follow up check at six weeks. A further three women who had not had a device inserted came for insertion at six weeks. 14 women attended earlier than six weeks due to noticing long threads or threads external to the vagina. One of these women was found to have a partial expulsion 
and the device was removed and a new IUC replaced. All others had the threads trimmed to $2 \mathrm{~cm}$ from the os. 
Figure 7-2 Outcomes of participants

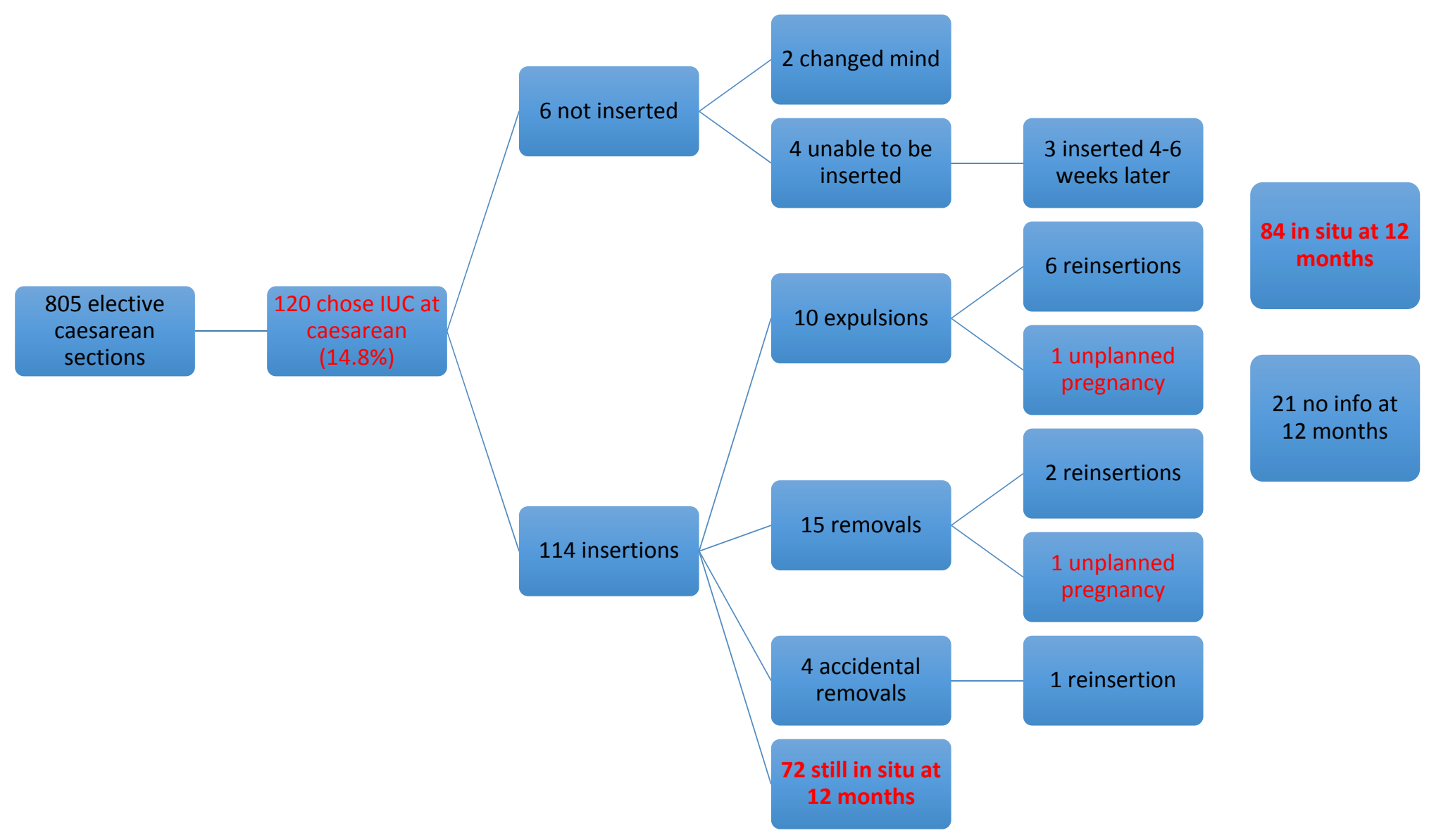


There were ten expulsions, seven by six weeks following insertion, one at seven weeks, one at 14 weeks and one at 28 weeks. This includes five partial expulsions (the stem of the device was seen in the cervical canal). All complete expulsions were noticed by women except in one case (details below). Of the ten expulsions, six women were fitted with a replacement IUC. In four cases, IUCs were removed accidentally by women. Two happened before women were seen for their six week thread check, when their threads had not been trimmed. Two others were pulled out with tampons. One woman had her device replaced.

There was one case of endometritis diagnosed at the six week check (pain plus $E$. coli infection confirmed on high vaginal swab). This was treated with oral antibiotics and the device was left in situ. Another device was removed at two days post insertion due to an initial suspicion of endometritis, but microbiological investigation was negative, and the woman was subsequently diagnosed with pyelonephritis. (A further device was inserted eight months later.)

Of complications that occurred, complications that had occurred by six weeks included six expulsions (three partial and one complete), two accidental removals and one case of endometritis. There were three removals before six weeks, one for suspected infection and two at patient request.

Of 97 women who attended believing they had an IUC in situ, threads were visible in 49 cases $(50 \%)$. Ultrasound confirmed intra-uterine placement in all but two women with missing threads. One woman was noted to have an intra cervical device, this was removed and alternative contraception started; this is counted in our data as a partial expulsion. Another woman had no device seen on ultrasound. Unfortunately despite multiple efforts she did not attend for abdominal x-ray. She did attend clinic six months after insertion with an unplanned pregnancy for a termination. Six women did not attend the six week thread check but were followed up by telephone and either reported that they could feel threads or had attended their GP (general practitioner) for a confirmation that the IUC threads were visible. Of four women that could not have an IUC inserted at caesarean, three of them attended for IUC insertion at six weeks. 


\subsubsection{Continuation and satisfaction rates}

Continuation rates are detailed in table 7.2. Percentages are detailed both from the total number of devices planned, using intention to treat and assuming that everyone lost to follow up is no longer using IUC for contraception, and as a percentage of number of women contacted. At 12 months 93 women were contactable, and information about a further six was gained from clinical databases, making a follow up rate of $82.5 \%$. As a proportion of all 117 women who got the device fitted either at caesarean $(n=114)$ or afterwards $(n=3), 71.8 \%$ were known to have a device in situ at 12 months $(\mathrm{n}=84)$

Table 7-2 Continued use of intrauterine contraception

\begin{tabular}{|l|l|l|l|l|l|}
\hline $\mathrm{n}=120$ & $\begin{array}{l}\text { At } \\
\text { caesarean } \\
\text { section }\end{array}$ & 6 weeks & $\begin{array}{l}3 \\
\text { months }\end{array}$ & $\begin{array}{l}6 \\
\text { months }\end{array}$ & $\begin{array}{l}12 \\
\text { months }\end{array}$ \\
\hline $\begin{array}{l}\text { Number of women } \\
\text { contacted/information } \\
\text { available }\end{array}$ & 120 & 112 & 109 & 105 & 99 \\
\hline Using IUC & 114 & $111 *$ & 96 & 91 & $84 * *$ \\
\hline $\begin{array}{l}\text { Continuation rates as } \\
\text { percentage of those } \\
\text { with known status }\end{array}$ & $95 \%$ & $99 \%$ & $88 \%$ & $87 \%$ & $85 \%$ \\
\hline $\begin{array}{l}\text { Continuation rates as } \\
\text { percentage of 120 } \\
\text { planned insertions }\end{array}$ & $95 \%$ & $91 \%$ & $80 \%$ & $76 \%$ & $70 \%$ \\
\hline $\begin{array}{l}* 114 \text { devices inserted at caesarean, 3 removals, 7 expulsions, } 4 \text { replacements, } \\
3 \text { new insertions }\end{array}$ & $\begin{array}{l}* * 72 \text { still in situ, 6 expulsions replaced, 2 removals replaced, 1 accidental } \\
\text { removal replaced, 3 new insertions }\end{array}$ \\
\hline
\end{tabular}

There have been 15 removals out of 117 devices inserted (12.8\%). 14 removals were of devices inserted at caesarean, one of a device inserted ten weeks postpartum. Removals have been from two days after insertion to 27 weeks, all were LNG-IUS devices. Reasons for removal included disliking bleeding patterns $(\mathrm{n}=5)$, headache $(\mathrm{n}=1)$, disliking a foreign body in the uterus $(\mathrm{n}=1)$,$) recurrent thrush (\mathrm{n}=1)$, pain during intercourse $(n=1)$, postpartum haemorrhage $(n=1)$, not liking the method 
$(n=1)$, believing the method affected milk production $(n=1)$ and wanting to conceive again ( $n=1,10$ months in situ). One removal was due to placement concerns. An ultrasonographer reported the IUC to be embedded in the myometrium, but it was removed easily and replaced. One woman whose device was removed due to an infection concern had IUC subsequently replaced. All removals were straightforward outpatient procedures, including in three cases where threads were not visible. Of note, one woman who chose to have her LNG-IUS removed and commenced a less effective method (the combined hormonal contraceptive patch) had an unintended pregnancy two months later.

After one year, 82 women still using the device were asked about their satisfaction with the device. $92.7 \%$ of them were 'very' or 'fairly' happy with the IUC $(n=76)$. Further satisfaction rates are detailed in table 7.3.

\section{Table 7-3 Satisfaction rates}

\begin{tabular}{|c|c|c|c|c|}
\hline \multicolumn{5}{|c|}{$\begin{array}{l}\text { Satisfaction with insertion taking place at the time of caesarean section }(n=114) \\
\text { (asked at } 6 \text { week follow up) }\end{array}$} \\
\hline Number of women asked & \multicolumn{4}{|l|}{98} \\
\hline Very happy & \multicolumn{4}{|l|}{$74(76 \%)$} \\
\hline Fairly happy & \multicolumn{4}{|l|}{$21(21 \%)$} \\
\hline Neither/nor & \multicolumn{4}{|l|}{$3(3 \%)$} \\
\hline Fairly unhappy & \multicolumn{4}{|l|}{0} \\
\hline Very unhappy & \multicolumn{4}{|l|}{0} \\
\hline \multicolumn{5}{|c|}{ Satisfaction with intrauterine contraception device } \\
\hline & $\begin{array}{l}6 \text { week } \\
\text { thread } \\
\text { check }\end{array}$ & $\begin{array}{l}3 \text { month } \\
\text { follow up }\end{array}$ & $\begin{array}{l}6 \text { month } \\
\text { follow up }\end{array}$ & $\begin{array}{l}12 \text { month } \\
\text { follow up }\end{array}$ \\
\hline $\begin{array}{l}\text { Number of women with IUC } \\
\text { in situ }\end{array}$ & $111^{*}$ & 96 & 91 & 84 \\
\hline $\begin{array}{l}\text { Number of women asked } \\
(=n)\end{array}$ & 99 & 92 & 89 & 82 \\
\hline Very happy & $42(42 \%)$ & $67(72 \%)$ & $61(69 \%)$ & $64(78 \%)$ \\
\hline Fairly happy & $43(43 \%)$ & $21(23 \%)$ & $22(25 \%)$ & $12(15 \%)$ \\
\hline Neither/nor & $14(14 \%)$ & $4(4 \%)$ & $4(4 \%)$ & $5(6 \%)$ \\
\hline Fairly unhappy & 0 & 0 & 0 & $1(1 \%)$ \\
\hline Very unhappy & 0 & 0 & $2(2 \%)$ & $1(1 \%)$ \\
\hline
\end{tabular}




\subsection{Discussion}

\subsubsection{Main findings}

Routinely offering insertion of IUC at the time of elective caesarean section was popular amongst women, with just over one in seven women choosing this option, the majority opting for an LNG-IUS. In addition, the study confirmed the low complication rate associated with insertion of IUC at this time and an expulsion rate in keeping with that of insertion of IUC in women who are not postpartum (Faculty of Sexual and Reproductive Healthcare, 2015b). Furthermore, satisfaction rates amongst women with IUC insertion at caesarean were high, and satisfaction and continuation with the method remained high by twelve months post insertion.

\subsubsection{Strengths and Limitations}

To our knowledge this is the first study from Western Europe to evaluate insertion of IUC at the time of elective caesarean section, routinely offered in a public health setting. Our loss to follow up was low, and reviewing regional databases minimised the risk of missing any serious complications associated with IUC insertion. In addition there is little existing data in the literature regarding women's acceptability of IUC insertion at this time. It is a significant strength of our research that we examined routine insertion of IUC in a setting where all contraception is free. A number of other studies have made IUC free for the purposes of research, in a setting where it would normally be an extra cost for patients. However in this NHS population, cost is not an incentive to choice of contraceptive method, so removing the barrier of attending a clinic at a later date in the postpartum period may have a proportionately greater impact on women's choice of IUC at caesarean.

This was an evaluation of a new service and so the findings are likely to represent uptake of IUC in similar public maternity settings. Our study is limited though, by the fact that this service evaluation relates to a single region. The percentage of women choosing IUC and the satisfaction rates may differ in other populations. We have limited data on removals, as only a small number of women have had their device removed. It is however reassuring that removal was straightforward in those women who requested it. 


\subsubsection{Discussion of results}

As has been argued elsewhere, the main advantage of insertion of IUC at caesarean section is the advantage it confers for uptake and continuity (Blumenthal and Goldthwaite, 2015). In addition the procedure is convenient both for women and health services, and avoids a potentially more difficult vaginal insertion (in some cases through a cervical os that has never dilated) weeks later. This is particularly relevant given the evidence that the risk of uterine perforation is increased in the first 36 weeks postpartum, with breastfeeding at time of insertion associated with a sixfold increased risk (Heinemann et al., 2015). The number of women choosing IUC as postpartum contraception was considerably higher than was previously found in a similar maternity population, when insertion at caesarean section was not routinely available (Heller et al., 2016).

Expulsion rates in our study were low, and when it did occur it was recognized in all but one case, and most women chose to have another device inserted. A potential challenge with IUC insertion at caesarean is that a significant proportion of women will not have visible threads at six weeks. In our study this was half of all cases. This indicates that an important component of making insertion of IUC at caesarean routine practice is a system ensuring rapid access to ultrasound scans to confirm that the IUC is in situ. This could be either at a hospital or clinic or by direct referral to sexual health centers that have ultrasound capabilities. A small number of women in our study presented with very long threads, but trimming the threads can be easily managed by any healthcare practitioner who is competent to conduct a cervical smear.

Insertion of IUC at caesarean section is a relatively straightforward procedure. Obstetricians are skilled operators and insertion of the IUC at caesarean is not complicated. Indeed it is less complicated than female sterilisation at the time of caesarean, and could in fact be offered to women as an alternative to this practice, particularly as sterilisation is a permanent procedure. Training of obstetricians was straightforward and required a small number of dedicated trainers only (the evaluation team). Training for midwives to ensure a basic knowledge about IUC and 
dispel any myths was also required, although some of this could be delivered online or in video format rather than face to face teaching sessions (Cameron et al., 2017).

Robust processes need to be in place in order for appropriate follow up post IUC insertion to succeed and so close working partnerships between maternity, sexual health services and GPs are important to make routine insertion of IUC at caesarean achievable and successful.

\subsection{Conclusion}

Chapter 6 set out the unmet need for contraception among the postpartum population, as shown by short inter-pregnancy intervals. Questionnaires conducted with patients suggest that these short intervals were not by choice, and the same questionnaires captured the openness of patients to considering immediate postpartum contraception. Chapter 7 outlines a practical, straightforward and viable approach to improving postpartum contraception for patients.

As demonstrated by the literature review in 7.2 , there is robust evidence that insertion of IUC at caesarean section is safe and carries a low complication rate. Randomised controlled trials demonstrate that continuation rates of IUC at six months are significantly higher in women randomised to insertion at the time of caesarean section (Lester et al., 2015; Levi et al., 2015; Braniff et al., 2015). Most RCTs are not powered to detect a difference in unplanned pregnancy rates or short inter-pregnancy intervals, however continuation rates of the most effective methods of contraception (Trussell, 2011) can act as a reasonable proxy for pregnancy rates in the absence of other data. Our complication rate was consistent with the existing evidence base, as was our continuation rate.

Set against the evidential background, our research aimed to investigate the feasibility of offering and delivering this service in a public health system, as well as its acceptability to patients. Over a period of six months, approximately one in seven women undergoing a caesarean section chose the option of IUC insertion. This was a new service, and it may be that this number rises as word and use of the service spreads. Already the popularity demonstrates the feasibility and acceptability of this service to patients, as does the high continuity and low withdrawal rates seen in this 
research. As immediate postpartum contraception also marks a culture change, it would also be useful to conduct some qualitative research, both with patients and staff, to gain a richer understanding of how participants feel about this change. In addition, we would like to continue to follow patients up for as long as five years, to capture information on their pregnancy spacing and on removals of devices inserted at caesarean. Longer-term information is currently scarce about this practice. Both aspects are possible areas for future research.

\subsubsection{Summary}

Routine provision of IUC at caesarean section is feasible with a simple training package for maternity staff. It is both safe, with low complication rates, and highly acceptable to women, with good uptake and good continuation rates. Any increase in LARC in the postpartum setting has the potential to prevent unintended pregnancy and enable women to achieve healthy inter-pregnancy intervals. This option should be routinely offered to women as part of good maternity care (Faculty of Sexual and Reproductive Healthcare, 2017). 


\section{Chapter 8 Conclusions, Clinical Implications and Directions for Future Research}

\subsection{Introduction}

This chapter will examine the research contained in this thesis in the context of expanding sexual healthcare provision. The successes and challenges of different aspects of research will be considered.

\subsection{Expanding contraception uptake to prevent unplanned pregnancy}

In Chapter 1 I introduced the idea of preventing unplanned pregnancy through avoiding one of three antecedents: non-use of contraception, incorrect or inconsistent use of contraception and contraceptive failure. This thesis describes research to address the first two of these precursors. Chapters 4 and 5 describe our pilot project examining users of the contraceptive injection receiving their injection from pharmacy. Although this does not in itself increase uptake, the survey described in chapter 3 suggests that if this was a feasible option, current non-users of contraception and users of less effective methods than the injection might be interested in this service (Heller et al., 2016). By increasing the convenience of the injection, both non-users and users of methods where inconsistent use is more likely (condoms and oral contraceptive pills) might be more likely to use this effective method. However, the pathway that is explored in chapters 4 and 5 of this thesis did not prove to be a feasible option for patients. The high level of withdrawals and the number of participants that chose to continue to visit the clinic even with the option of pharmacy attendance suggests that this was not an attractive option for many people.

As discussed in chapters 6 and 7, provision of contraception at childbirth is an ideal time to offer postpartum women, who may be unsure about what contraceptive methods they can use, (Murphy and British Pregnancy Advisory Service, 2014) the most effective methods. Since the start of my research fellowship, a great deal more evidence has been published supporting this idea. Research consistently demonstrates that providing contraception at the time of childbirth, particularly in the 
form of the most effective LARC methods, is acceptable to women, straightforward for clinicians, and cost-effective. Notably, receiving LARC post-pregnancy leads to longer inter-pregnancy intervals (Harney et al., 2017). Women are much more likely to receive LARC if they are offered it in the immediate postpartum period, whether that means an IUD at the time of delivery (Lester et al., 2015; Levi et al., 2015; Foreit et al., 1993), or a contraceptive implant inserted immediately postpartum (Tocce et al., 2012b). The publication of a Best Practice Paper in Postpartum Family Planning by the Royal College of Obstetricians in 2015 (Royal College of Obstetricians and Gynaecologists, 2015), and the guidance on Contraception after Pregnancy from the Faculty of Sexual and Reproductive Health in 2017 (Faculty of Sexual and Reproductive Healthcare, 2017) are beginning to alter the landscape of postpartum contraception significantly.

It is easy to identify why immediate postpartum contraception has gained increasing attention not only from clinicians but also from government and legislators (The Scottish Government, 2015; Kramer, 2017). What evidence there is shows that postpartum women in the United Kingdom lack information about postpartum fertility and when to start a contraceptive method (Murphy and British Pregnancy Advisory Service, 2014). Prior to the new guidelines neither postpartum midwives (McCance and Cameron, 2014) nor GPs at the postpartum check (Lunniss et al., 2016) prioritised contraceptive advice. As a result postpartum women may end up being non-users of contraception, and at high risk of unplanned pregnancy. An intervention at the time of delivery offers the opportunity for women to receive any contraceptive method. Interval postpartum insertion (four to six weeks after birth) of intrauterine contraception in particular may require additional visits (Lunniss et al., 2016), which are a reason women do not receive IUC (Zerden et al., 2015). All LARC methods require insertion by someone who has been trained, a fact that can sometimes be a barrier to improving uptake (Kavanaugh et al., 2013). However maternity staff are likely to have many of the requisite basic skills such as pelvic examination and familiarity with inserting devices such as scalp electrodes through the cervix. Fostering interest and inclination in maternity HCPs to undertake some brief additional training would enable women to be provided with all methods, including LARC, as per new guidelines (Faculty of Sexual and Reproductive 
Healthcare, 2017). In this way fertile women who might be non-users, or users of less effective methods, can have access to the methods with the least risk of incorrect or inconsistent use, and therefore the best chance at avoiding unplanned pregnancy.

Chapter 6 showed that when asked only a small number of women were planning on using LARC in the postpartum period. However a much greater number were open to immediate insertion, with almost one in three saying that they would use intrauterine contraception if it could be fitted immediately (Heller et al., 2016). Most women questioned were planning on using condoms or pills, in keeping with the popular methods in the United Kingdom (Cea Soriano et al., 2014a; Lader, 2009). However, among modern contraceptive methods, these methods are most likely to be associated with unplanned pregnancy, due to their higher risk of inconsistent and/or imperfect use (Jones et al., 2002; Rasch, 2002). Increasing postpartum uptake of LARC would therefore also address the problem of unplanned pregnancy by reducing pregnancies associated with inconsistent and imperfect use of contraception. Chapter 7 demonstrates the feasibility and acceptability of a straightforward way to offer LARC at delivery for women delivering by caesarean section.

\subsection{The crucial nature of convenience}

In my research examining the feasibility of participants receiving the contraceptive injection at the community pharmacy, participants' comments emphasise difficulties of access. Barriers to attending the pharmacy included lack of information when contacting the pharmacy and limited availability of the pharmacist trained to provide the service. Those patients who did comment positively on the pharmacy all mentioned convenience and ease as being a significant part of why the experience was a good one, with comments such as "easy and positive experience", "flexible with times" and "so much more convenient than visiting the nurse". Chapter 3 outlines a questionnaire conducted in the sexual health clinic, which demonstrates the anticipation that pharmacy attendance would be more convenient, with better opening hours, a quicker appointment, and easier to access premises (Heller and Cameron, 2016). 
What patients were looking for was a more convenient experience, where obtaining contraception fitted seamlessly into their daily lives. The extra work involved in contacting the pharmacy more than once and negotiating appointments during limited opening hours clearly impacted upon participants, and it seems likely that this is partly responsible for the high numbers of participants who left the study. The importance of convenience is echoed in the literature asking women about advantages of pharmacy access. In a survey of 811 American women about pharmacy access to contraception, $85 \%$ believed they would benefit from convenient hours of pharmacies, $84 \%$ from convenient locations, $82 \%$ from saved time, and $74 \%$ from the absence of the need for a clinician appointment (Landau et al., 2006). In a survey of 651 American women seeking abortion services, 514 expressed support for over-the-counter access to contraception. The most commonly cited reason was convenience, given as a reason by $76 \%$ (Grindlay et al., 2014). Focus groups with young women and women of colour, again in the United States, emphasise once more that convenience is the most important perceived advantage of over-the-counter access, with one participant stating that to get hormonal contraception from a pharmacy would make it "almost as convenient as a condom" (Baum et al., 2016).

For some participants in the research described in chapters 4 and 5, the experience of making an appointment with the pharmacist with limited availability did not come close to the experience of buying condoms from a pharmacy, and therefore their involvement fell short of offering the advantages of convenience both anticipated by participants and in the literature. The contraceptive injection does not feature prominently in research looking at method failure leading to unplanned pregnancy (Schünmann and Glasier, 2006; Rasch, 2002; Jones et al., 2002). However this is likely to be because of its relative unpopularity rather than its efficacy, in 2008 it was the method for just $2.4 \%$ of contraceptive users (Cea Soriano et al., 2014a). It is clearly evident that if patients are unable to attend their injection provider in a timely fashion, they will be at risk of unplanned pregnancy.

Rather than replicating a current contraceptive service delivery method in the pharmacy (making an appointment to receive method) it may therefore be more 
effective to concentrate on over-the-counter access for contraception. The evidence for this is detailed in Chapter 2, however there is very little evidence assessing the acceptability of this idea among a UK public. In addition, over-the-counter access is likely to be available only for short-acting methods like the pill, the patch, and possibly the vaginal ring (though this last method is not popular in the UK) (Lader, 2009). All of the existing literature about the acceptability of contraception OTC focuses on the pill, patch and ring, which is unsurprising as both the contraceptive implant and intrauterine contraception need trained providers to insert. Making less effective methods available at pharmacy might therefore work to reduce the uptake of LARC, as women may attend pharmacies as their primary place to receive contraception. OTC access to contraception would need to be accompanied by close partnership links between pharmacy and sexual health clinics, and pharmacists must be aware of comparative effectiveness rates of LARC methods. The research described in Clement and Mansour, where pharmacists received intensive education about all emergency contraception options including the copper IUD, and a rapid access referral pathway was established for pharmacists, is an ideal model to consider here (Clement and Mansour, 2014).

Self-administration of the contraceptive injection clearly offers the most convenient way for patients to access this particular method, and it is to be welcomed that the SC-DMPA used in our study is now licensed for self-injection (Faculty of Sexual and Reproductive Healthcare, 2015a). The available evidence is that selfadministration is feasible and straightforward for patients, with high satisfaction rates, although of note no study has to date shown that it improves continuation rates compared to receiving an injection by a healthcare professional (Prabhakaran and Sweet, 2012; Cameron et al., 2012b; Beasley et al., 2014; Cover et al., 2017).

Offering patients insertion of intrauterine contraception at the time of caesarean section presented them with a clear advantage in terms of convenience. Evidence shows that women who want a LARC method fitted in the weeks after giving birth may need to attend two visits with a healthcare practitioner, in addition to the routine six-week check, in order to get intrauterine contraception fitted (Lunniss et al., 2016). Attendance for fitting of intrauterine contraception postpartum may be as low 
as $50 \%$ (Ogburn et al., 2005), and women identify extra visits as a barrier to insertion, even when they have decided that they would like to use a LARC method (Zerden et al., 2015). By contrast, almost all the women in the study described in Chapter 7 did attend for the check of their IUC inserted at caesarean section, suggesting that women may prioritise a check up for an existing method of contraception differently to a visit to start a new method.

RCTs looking at insertion of IUC at caesarean section demonstrate that women who are randomised to receive IUC at the time of caesarean are more likely to receive it than those who are randomised to receive it at a follow up visit (Lester et al., 2015; Levi et al., 2015; Braniff et al., 2015). Unsurprisingly, a recent analysis of women who plan to use LARC after delivery has demonstrated that those who do not receive LARC are more likely to have short inter-pregnancy intervals (Harney et al., 2017). In addition a very large cohort study of 61,448 women in six European countries examining IUC insertion found that both breastfeeding at the time of insertion and an interval of $\leq 36$ weeks since last delivery were independently associated with an increased risk of uterine perforation (Heinemann et al., 2015). There are no documented perforations when IUC is inserted at caesarean section. Immediate postpartum IUC may therefore not only be more convenient, it may carry fewer risks.

The high continuation and satisfaction rates described in chapter 7 lend weight to the idea that opportunistic delivery of contraception at the time of childbirth works equally as well as the well-documented opportunistic delivery of contraception at the time of termination of pregnancy (Goodman et al., 2008; Rose and Lawton, 2012; Cameron et al., 2012a; Church et al., 2010; Royal College of Obstetricians and Gynaecologists, 2011). Although these areas have traditionally been viewed differently for contraception provision, with the advent of new guidelines that comprehensively address delivery of contraception after pregnancy, whether the outcome of the pregnancy is childbirth, abortion, miscarriage or ectopic pregnancy, the medical norms around contraception are beginning to change (Faculty of Sexual and Reproductive Healthcare, 2017). 


\subsection{Ideas for future research}

The new guidelines on contraception delivery after pregnancy identify all pregnancies as a time to discuss contraception, regardless of outcome. This includes miscarriage and ectopic pregnancy. Evidence from Scotland shows the percentage of unintended pregnancies was almost the same for women at the miscarriage clinic as at the antenatal clinic (11.9\% versus 7.7\% respectively) (Lakha and Glasier, 2006). Data from the Natsal-3 survey in 2010-2012 demonstrates that of women whose pregnancy ended in miscarriage, $35.3 \%$ were planned pregnancies, $31.1 \%$ were ambivalent and 33.6\% were unplanned pregnancies (Wellings et al., 2013). These figures would suggest that women experiencing miscarriage are at an equal if not higher risk of the pregnancy being unintended as women having a live birth. They are therefore a cohort in whom opportunistic contraception would be appropriate.

Although some clinicians might assume that after a miscarriage women would want to become pregnant again quickly, there is no data about how many women go on to have an unplanned pregnancy that then ends in abortion after a miscarriage. Given the high number of unplanned pregnancies ending in miscarriage the number is likely to be substantial. These could be avoided if effective contraception was started after miscarriage for those women who wanted it. However there is essentially no literature about the feasibility, acceptability or effectiveness of offering women contraception at the time of miscarriage (Faculty of Sexual and Reproductive Healthcare, 2017). This is a rich area for further research, both qualitative and quantitative. Qualitative research with midwives in maternity care revealed that some felt their poor knowledge of contraception was a barrier to contraceptive discussions (McCance and Cameron, 2014). Similar research could be usefully conducted in miscarriage services, to identify perceived barriers and opportunities, as well as with women who have experienced miscarriage, in order to identify ways to bring up contraception at a sensitive time.

The period after an ectopic pregnancy may be perceived as an easier time to introduce the idea of contraception to women, as the clinical advice is to delay pregnancy for three months (Faculty of Sexual and Reproductive Healthcare, 2017). However, there is a similar lack of research on starting contraception immediately 
after ectopic pregnancy in order to avoid future unplanned pregnancies, and therefore a similar opportunity to learn more.

There is evidence that insertion of IUC after a vaginal birth offers many of the advantages of convenience, time saving, cost-effectiveness and attractiveness to patients as insertion of IUC at caesarean section, even taking into account that the expulsion rate is higher (Chen et al., 2009; Kapp and Curtis, 2009; Chen et al., 2010; Mwalwanda and Black, 2013; Lopez et al., 2015; Washington et al., 2015; Goldthwaite and Shaw, 2015; Eggebroten et al., 2017). To date there has been no research with a UK patient population examining this practice, although an observational study is now beginning in Edinburgh (Wellbeing of Women, 2016). An RCT examining this practice, large enough to detect a difference in unplanned pregnancy rates or short inter-pregnancy intervals, would give the highest level of evidence about this exciting possibility.

One of the reasons that participants withdrew from our research investigating contraceptive injections at the community pharmacy was that they preferred selfinjection. To date there has been one RCT examining self-injection of DMPA compared to clinic use, with 86 women randomised to self-injection and 46 to clinic administration. There was no significant difference in continuation rates at the end of one year $(p=0.47)$ (Beasley et al., 2014). The study was not powered to investigate pregnancy rates. The percentage of women experiencing an unintended pregnancy in the first year of using Depo-Provera ${ }^{\circledR}$ is 6\% with typical use (Trussell, 2011). This rate might be lower in women who are self-injecting and are therefore at less risk of missing injections. Any RCT powered for a difference in unplanned pregnancy rates would therefore have to be very large. However a larger trial could more robustly confirm or refute any significant difference in continuation rates. The existing RCT (Beasley et al., 2014) also took place in the United States, so repeating this trial in the United Kingdom would be a useful way to determine whether UK women respond differently to the availability of self-injection. A recent systematic review found only this RCT, a pilot cohort study from Scotland and a prospective cohort study examining a combined injection of DMPA and estradiol. It concludes that there is no evidence that either continuation rates or satisfaction are higher with 
either clinic or self-administration (Kim et al., 2017). More research, looking at ideally unplanned pregnancy, but if not then at continuation rates and patient satisfaction would be a very useful addition to the literature. Self-administration is likely to be resource saving for the National Health Service and therefore any research would benefit from a cost-effectiveness analysis too.

\subsection{Final thoughts}

Novel service models can offer patients improved access contraception. Those which present the most straightforward and convenient ways of delivery are the most likely to be successful in enabling patients to either begin contraception, or to begin using a method with less risk of imperfect use. The post-pregnancy period is gaining momentum both globally and in the United Kingdom as an ideal time to offer contraception, and LARC methods have the best evidence for preventing unplanned pregnancy and increasing inter-pregnancy intervals.

Before any new service models are adopted and funded a rigorous examination of the literature will enable targeted and well-designed research trials to investigate service delivery. Definitive research should then be conducted, ideally using RCTs, although this is not always practical in the field of contraception. Similarly endpoints of unplanned pregnancy rates, whilst providing the most useful data, may be difficult when examining what is still a rare event. Nonetheless, rigorous research is our best approach to understanding how to best deliver contraception. Improved uptake of the most effective contraceptive methods offers the best chance at avoiding unplanned pregnancy for society and for our patients. 


\section{References}

(1967). Abortion Act 1967.

Ajuwon A, Imaledo J, Oshiname F \& Ajayi O. (2013) Delivery of injectable contraceptives by patient medicine vendors in rural communities in Oyo state, Nigeria. FHI 360,

Ajzen I (1991). The theory of planned behavior. Organizational Behavior and Human Decision Processes, 50(2), 179-211.

Akol A, Chin-Quee D, Wamala-Mucheri P, Namwebya JH, Mercer SJ \& Stanback J (2014). Getting closer to people: family planning provision by drug shops in Uganda. Global Health: Science and Practice, 2(4), 472-481.

Anderson C \& Blenkinsopp A (2006). Community pharmacy supply of emergency hormonal contraception: a structured literature review of international evidence. Human Reproduction, 21(1), 272-284.

Anderson C, Blenkinsopp A \& Armstrong M (2004). Feedback from community pharmacy users on the contribution of community pharmacy to improving the public's health: a systematic review of the peer reviewed and non-peer reviewed literature 1990-2002. Health Expectations, 7(3), 191-202.

Anderson C, Blenkinsopp A \& Armstrong M. (2009) The contribution of community pharmacy to improving the public's health. Summary report of the literature review 1990-2007. PharmacyHealthLink, London.

Anderson C \& Thornley T (2014). "It's easier in pharmacy": why some patients prefer to pay for flu jabs rather than use the National Health Service. BMC Health Services Research, 14, 35.

Anderson C (1998). Health promotion by community pharmacists: consumers' views. International Journal of Pharmacy Practice, 6(1), 2-12.

Anderson C, Bissell P, Sharma S \& Sharma R (2001). Supplying emergency hormonal contraception in the pharmacy: the perspectives of service users. International Journal of Pharmacy Practice, 9(S1), R56.

Anderson C, Blenkinsopp A \& Armstrong M. (2005) The contribution of community pharmacy to improving the public"s health: evidence from the peer-reviewed literature 2001-2004. PharmacyHealthLink, London.

Aneblom G, Lundborg CS, Carlsten A, Eurenius K \& Tydén T (2004). Emergency contraceptive pills over-the-counter: practices and attitudes of pharmacy and nursemidwife providers. Patient Education and Counseling, 55(1), 129-135.

Ayoola AB, Nettleman M \& Brewer J (2007). Reasons for unprotected intercourse in adult women. Journal of Women's Health, 16(3), 302-310.

Bacon L, Savage I, Cook S \& Taylor B (2003). Training and supporting pharmacists to supply progestogen-only emergency contraception. Journal of Family Planning and Reproductive Health Care, 29(2), 17-22. 
Ball DE, Marafie N \& Abahussain E (2006). Awareness and perceptions of emergency contraception among retail pharmacists in Kuwait. Pharmacy World and Science, 28(2), 101-106.

Barlassina L (2015). Views and attitudes of oral contraceptive users towards their availability without a prescription in the Republic of Ireland. Pharmacy Practice, 13(2), 565.

Barrett G, Smith SC \& Wellings K (2004). Conceptualisation, development, and evaluation of a measure of unplanned pregnancy. Journal of Epidemiology and Community Health, 58(5), 426-433.

Battle M, Kueter T, Caraway J, Payakachat N, Stafford R \& Ragland D (2013). Customer satisfaction with emergency contraception counseling in a retail pharmacy setting. Proceedings from The American Pharmacists Association Conference, Los Angeles, USA.

Baum S, Burns B, Davis L, Yeung M, Scott C, Grindlay K et al. (2016). Perspectives among a diverse sample of women on the possibility of obtaining oral contraceptives over the counter: a qualitative study. Women's Health Issues, 26(2), 147-152.

Beasley A, O’Connell White K, Cremers S \& Westhoff C (2014). Randomized clinical trial of self versus clinical administration of subcutaneous depot medroxyprogesterone acetate. Contraception, 89(5), 352-356.

Bednarek PH, Creinin MD, Reeves MF, Cwiak C, Espey E \& Jensen JT (2011). Immediate versus delayed IUD insertion after uterine aspiration. New England Journal of Medicine, 364(23), 2208-2217.

Bennett W, Petraitis C, D'Anella A \& Marcella S (2003). Pharmacists' knowledge and the difficulty of obtaining emergency contraception. Contraception, 68(4), 261267.

Berry HJ, Shaw M \& Willis SC (2016). An evaluation of the ASC (action and support in pregnancy in Coventry) emergency hormonal contraception scheme. Proceedings from Health Services Research and Pharmacy Practice Conference, Reading, United Kingdom.

Bhutta SZ, Butt IJ \& Bano K (2011). Insertion of intrauterine contraceptive device at caesarean section. Journal of the College of Physicians and Surgeons - Pakistan, 21(9), 527-530.

Bissell P \& Anderson C (2003). Supplying emergency contraception via community pharmacies in the UK: reflections on the experiences of users and providers. Social Science \& Medicine, 57(12), 2367-2378.

Bissell P, Savage I \& Anderson C (2006). A qualitative study of pharmacists' perspectives on the supply of emergency hormonal contraception via patient group direction in the UK. Contraception, 73(3), 265-270.

Black K, Anderson C, Kubba A \& Wellings K (2009). Involving pharmacists in sexual health research: experience from an emergency contraception study. Journal of Family Planning and Reproductive Health Care, 35(1), 41-43. 
Black KI, Mercer CH, Kubba A \& Wellings K (2008). Provision of emergency contraception: a pilot study comparing access through pharmacies and clinical settings. Contraception, 77(3), 181-185.

Blumenthal PD \& Goldthwaite LM (2015). Intrauterine device insertion during cesarean delivery: the rising tide of the postdelivery intrauterine device. Obstetrics \& Gynecology, 126(1), 1-2.

Boots.com. (2016). Health pharmacy advice: winter flu jab services. Retrieved 23rd March, 2017, from http://www.boots.com/health-pharmacyadvice/vaccinations/flujab.

Borrego ME, Short J, House N, Gireesh G, Naik R \& Cuellar D (2006). New Mexico pharmacists' knowledge, attitudes, and beliefs toward prescribing oral emergency contraception. Journal of the American Pharmacists Association, 46(1), 33-43.

Borrero S, Nikolajski C, Steinberg JR, Freedman L, Akers AY, Ibrahim S et al. (2015). "It just happens": a qualitative study exploring low-income women"s perspectives on pregnancy intention and planning. Contraception, 91(2), 150-156.

Braniff K, Gomez E \& Muller R (2015). A randomised clinical trial to assess satisfaction with the levonorgestrel releasing intrauterine system inserted at caesarean section compared to postpartum placement. Australian and New Zealand Journal of Obstetrics and Gynaecology, 55(3), 279-283.

Brant A, White K \& St. Marie P (2014). Pharmacy availability of ulipristal acetate emergency contraception: an audit study. Proceedings from The North American Forum on Family Planning, Miami, USA.

Brass EP (2001). Changing the status of drugs from prescription to over-the-counter availability. New England Journal of Medicine, 345(11), 810-816.

British Pregnancy Advisory Service. (2012). Mumsnet/BPAS survey shows gaps in contraception care for new mums. Retrieved 28th September, 2014, from https://www.bpas.org/about-our-charity/press-office/press-releases/mumsnetbpassurvey-shows-gaps-in-contraception-ca/.

Brown D, Portlock J \& Rutter P (2012). Review of services provided by pharmacies that promote healthy living. International Journal of Clinical Pharmacy, 34(3), 399409.

Bullock H, Steele S, Kurata N, Tschann M, Elia J, Kaneshiro B et al. (2015). "I need to look that up. I've never filled it before": information from pharmacy staff regarding ullipristal acetate. Proceedings from The North American Forum on Family Planning, Chicago, USA.

Burke HM, Mueller MP, Packer C, Perry B, Bufumbo L, Mbengue D et al. (2014a). Provider acceptability of Sayana Press: results from community health workers and clinic-based providers in Uganda and Senegal. Contraception, 89(5), 368-373.

Burke HM, Mueller MP, Perry B, Packer C, Bufumbo L, Mbengue D et al. (2014b). Observational study of the acceptability of Sayana Press among intramuscular DMPA users in Uganda and Senegal. Contraception, 89(5), 361-367.

California State Legislature Assembly (2013). Pharmacy practice (S 493) 2013-2014. 
Cameron ST (2014). Postabortal and postpartum contraception. Best Practice \& Research Clinical Obstetrics and Gynecology, 28(6), 871-880.

Cameron ST, Glasier A, Chen ZE, Johnstone A, Dunlop C \& Heller R (2012a). Effect of contraception provided at termination of pregnancy and incidence of subsequent termination of pregnancy. British Journal of Obstetrics and Gynaecology, 119(9), 1074-1080.

Cameron ST, Glasier A, Muir A, Scott G, Johnstone A, Quarrell H et al. (2010). Expedited partner therapy for Chlamydia trachomatis at the community pharmacy. British Journal of Obstetrics and Gynaecology, 117(9), 1074-1079.

Cameron ST, Glasier A \& Johnstone A (2012b). Pilot study of home selfadministration of subcutaneous depo-medroxyprogesterone acetate for contraception. Contraception, 85(5), 458-464.

Cameron ST, Gordon R \& Glasier A (2012c). The effect on use of making emergency contraception available free of charge. Contraception, 86(4), 366-369.

Cameron ST, Craig A, Sim J, Gallimore A, Cowan S, Dundas K et al. (2017). Feasibility and acceptability of introducing routine antenatal contraceptive counseling and provision of contraception after delivery in an NHS setting: The APPLES pilot evaluation. British Journal of Obstetrics and Gynaecology, DOI: 10.1111/1471-0528.14674, [Epub ahead of print].

Carstairs V \& Morris R. (1991) Deprivation and Health in Scotland. Aberdeen University Press, Aberdeen.

Cea Soriano L, Garcia Rodriguez LA, Machlitt A \& Wallander MA (2014a). Use of prescription contraceptive methods in the UK general population: a primary care study. British Journal of Obstetrics and Gynaecology, 121(1), 53-61.

Cea Soriano L, Wallander M-A, Andersson S, Filonenko A \& García Rodríguez LA (2014b). Use of long-acting reversible contraceptives in the UK from 2004 to 2010: Analysis using The Health Improvement Network Database. The European Journal of Contraception \& Reproductive Health Care, 19(6), 439-447.

Celen S, Möröy P, Sucak A, Aktulay A \& Danişman N (2004). Clinical outcomes of early postplacental insertion of intrauterine contraceptive devices. Contraception, 69(4), 279-282.

Celen S, Sucak A, Yildiz Y \& Danisman N (2011). Immediate postplacental insertion of an intrauterine contraceptive device during cesarean section.

Contraception, 84(3), 240-243.

Chen BA, Hayes JL, Hohmann HL, Perriera LK, Reeves MF \& Creinin MD (2009). A randomized trial of postplacental compared to delayed insertion of the levonorgestrel-releasing intrauterine device after vaginal delivery. Contraception, 80(2), 205.

Chen BA, Reeves MF, Hayes JL, Hohmann HL, Perriera LK \& Creinin MD (2010). Postplacental or delayed insertion of the levonorgestrel intrauterine device after vaginal delivery: a randomized controlled trial. Obstetrics \& Gynecology, 116(5), 1079-1087. 
Chi IC, Gao J, Siemens AJ \& Waszak CS (1986). IUD insertion at cesarean section the Chinese experience. Advances in Contraception, 2(2), 145-153.

Chi IC, Zhou SW, Balogh S \& NG K (1984). Post-cesarean section insertion of intrauterine devices. American Journal of Public Health, 74(11), 1281-1282.

Chin-Quee D, Hinson L, L'Engle KL, Otterness C \& Janowitz B (2012). Bridge over troubled waters: considerations in transitioning emergency contraceptive users to hormonal methods. Contraception, 85(4), 363-368.

Chin-Quee DS, Cuthbertson C \& Janowitz B (2006). Over-the-counter pill provision: evidence from Jamaica. Studies in Family Planning, 37(2), 99-110.

Chin-Quee DS, Wedderburn M, Otterness C, Janowitz B \& Chen-Mok M (2010). Bridging emergency contraceptive pill users to regular contraception: results from a randomized trial in Jamaica. Contraception, 81(2), 133-139.

Chin KL (2011). Sexual/reproductive health and the pharmacist: what is known and what is needed. Journal of Pharmaceutical Health Services Research, 2(2), 65-69.

Church E, Sengupta S \& Chia KV (2010). The contraceptive implant for long acting reversible contraception in patients undergoing first trimester medical termination of pregnancy. Sexual \& Reproductive Healthcare, 1(3), 105-109.

Cleland K, Bass J \& Foster AM (2016). Access to emergency contraception in the over-the-counter era. Women's Health Issues, 26(6), 622-627.

Clement KM \& Mansour DJ (2014). Improving uptake of the copper intrauterine device for emergency contraception by educating pharmacists in the community. Journal of Family Planning and Reprodutive Health Care, 40(1), 41-45.

Cohen MM, Dunn S, Cockerill R \& Brown TER (2004). Using a secret shopper to evaluate pharmacist provision of emergency contraception: satisfaction levels high, with privacy a concern. Canadian Pharmacists Journal / Revue des Pharmaciens du Canada, 137(1), 28-33.

Connolly A, Thorp J \& Pahel L (2005). Effects of pregnancy and childbirth on postpartum sexual function: a longitudinal prospective study. International Urogynecology Journal, 16(4), 263-267.

Cooper RJ, Bissell P \& Wingfield J (2008). Ethical, religious and factual beliefs about the supply of emergency hormonal contraception by UK community pharmacists. Journal of Family Planning and Reproductive Health Care, 34(1), 4750 .

Cover J, Namagembe A, Tumusiime J, Lim J, Drake JK \& Mbonye AK (2017). A prospective cohort study of the feasibility and acceptability of depot medroxyprogesterone acetate (DMPA) administered subcutaneously through selfinjection. Contraception, 95(3), 306-311.

Curry CL, Iverson R, Rindos N \& Sonalkar S (2012). Immediate postplacental IUD placement after cesarean and vaginal deliveries at an academic training center. Contraception, 86(2), 176. 
D Souza R \& Bounds W (2001). Over-the-counter emergency hormonal contraception: a survey of pharmacists' and general practitioners' knowledge, use and views. Pharmaceutical Journal, 266, 293-297.

Davidson AR, Kalmuss D, Cushman LF, Romera D, Heartwell S \& Rulin M (1997). Injectable contraceptive discontinuation and subsequent unintended pregnancy among low-income women. American Journal of Public Health, 87(9), 1532-1534.

Dennis A \& Grossman D (2012). Barriers to contraception and interest in over the counter access among low income women: a qualitative study. Perspectives on Sexual and Reproductive Health, 44(2), 84-91.

Department of Health. (2001) Better Prevention, Better Services, Better Sexual Health - the National Strategy for Sexual Health and HIV. Department of Health, London.

Department of Health. (2004) Choosing Health: Making Healthier Choices Easier. Department of Health, London.

Department of Health. (2005) Choosing Health through Pharmacy. Department of Health, London.

Department of Health. (2013) A Framework for Sexual Health Improvement in England. Department of Health, London.

Dias T, Abeykoon S \& Kumarasiri... S (2015). Use of ultrasound in predicting success of intrauterine contraceptive device insertion immediately after delivery. Ultrasound in Obstetrics \& Gynecology, 46(1), 104-108.

Donovan GR \& Paudyal V (2016). England's Healthy Living Pharmacy (HLP) initiative: Facilitating the engagement of pharmacy support staff in public health. Research in Social and Administrative Pharmacy, 12(2), 281-292.

Downing SG, Payze C, Doyle-Adams S \& Gorton C (2011). Emergency contraception over-the-counter: practices and attitudes of pharmacists and pharmacy assistants in far North Queensland. Australian and New Zealand Journal of Obstetrics and Gynaecology, 51(6), 527-531.

Dunn S, Brown TER, Cohen MM, Cockerill R, Wichman K, Weir N et al. (2003). Pharmacy provision of emergency contraception: the Ontario emergency contraception pilot project. Journal of Obstetrics and Gynaecology Canada, 25(11), 923-930.

Eades CE, Ferguson JS \& O'Carroll RE (2011). Public health in community pharmacy: a systematic review of pharmacist and consumer views. BMC Public Health, 11, 582.

Eggebroten JL, Sanders JN \& Turok DK (2017). Immediate postpartum intrauterine device and implant program outcomes: A prospective analysis. American Journal of Obstetrics \& Gynecology, [Epub ahead of print], doi: 10.1016/j.ajog.2017.03.015.

Elsedeek MS (2012). Puerperal and menstrual bleeding patterns with different types of contraceptive device fitted during elective cesarean delivery. International Journal of Gynaecology \& Obstetrics, 116(1), 31-34. 
Elsedeek MSE (2015). Five-year follow-up of two types of contraceptive device fitted during elective cesarean delivery. International Journal of Gynaecology \& Obstetrics, 130(2), 179-182.

Evans A, Hinchcliffe A \& Jenkins N (2013). Can providing NHS flu vaccination in community pharmacies reach people who otherwise would not get vaccinated? Experiences from the national community pharmacy seasonal influenza service in Wales. Proceedings from The Royal Pharmaceutical Society Annual Conference, Birmingham, UK.

Faculty of Sexual and Reproductive Healthcare. (2009a) Postnatal Sexual and Reproductive Health. Faculty of Sexual \& Reproductive Healthcare, London.

Faculty of Sexual and Reproductive Healthcare. (2009b) UK Medical Eligibility Criteria for Contraceptive Use 2009. Faculty of Sexual \& Reprodcutive Healthcare, London.

Faculty of Sexual and Reproductive Healthcare. (2012) Emergency Contraception Clinical Guidance. Faculty of Sexual \& Reproductive Health, London.

Faculty of Sexual and Reproductive Healthcare. (2013) New Product Review from the Clinical Effectiveness Unit. Subcutaneous Depo Medroxyprogesterone Acetate (Sayana Press). Faculty of Sexual \& Reproductive Healthcare, London.

Faculty of Sexual and Reproductive Healthcare. (2015a) Clinical Effectiveness Unit Statement on Self-Administration of Sayana Press. Faculty of Sexual \& Reproductive Healthcare, London.

Faculty of Sexual and Reproductive Healthcare. (2015b) Intrauterine Contraception Clinical Guidance. Faculty of Sexual \& Reproductive Healthcare, London.

Faculty of Sexual and Reproductive Healthcare. (2016) UK Medical Eligibility Criteria for Contraceptive Use 2016. Faculty of Sexual \& Reproductive Healthcare, London.

Faculty of Sexual and Reproductive Healthcare. (2017) FSRH Guideline: Contraception after Pregnancy. Faculty of Sexual \& Reproductive Healthcare, London.

Fakih S, Batra P, Gatny HH, Kusunoki Y, Barber JS \& Farris KB (2015). Young women's perceptions and experiences with contraception supply in community pharmacies. Journal of the American Pharmacists Association, 55(3), 255-264.

Famiyeh I-M \& McCarthy L (2017). Pharmacist prescribing: A scoping review about the views and experiences of patients and the public. Research in Social and Administrative Pharmacy, 13(1), 1-16.

Farris KB, Ashwood D, McIntosh J, DiPietro NA, Maderas NM, Landau SC et al. (2010). Preventing unintended pregnancy: pharmacists' roles in practice and policy via partnerships. Journal of the American Pharmacists Association, 50(5), 604-612.

Finer LB \& Henshaw SK (2006). Disparities in rates of unintended pregnancy in the United States, 1994 and 2001. Perspectives on Sexual and Reproductive Health, 38(2), 90-96. 
Folkes L, Graham A \& Weiss M (2001). A qualitative study of the views of women aged 18-29 on over-the-counter availability of hormonal emergency contraception. Journal of Family Planning and Reproductive Health Care, 27(4), 189-192.

Foreit KG, Foreit JR, Lagos G \& Guzman A (1993). Effectiveness and costeffectiveness of postpartum IUD insertion in Lima, Peru. International Family Planning Perspectives, 19(1), 19-33.

Forrest JD (1994). Epidemiology of unintended pregnancy and contraceptive use. American Journal of Obstetrics and Gynecology, 170(5), 1485-1489.

French AC \& Kaunitz AM (2007). Pharmacy access to emergency hormonal contraception in Jacksonville, FL: a secret shopper survey. Contraception, 75(2), 126-130.

French RS, Mercer CH, Johnson AM, Fenton KA, Erens B \& Wellings K (2009). Use of contraceptive services in Britain: findings from the second National Survey of Sexual Attitudes and Lifestyles (Natsal--2). Journal of Family Planning and Reproductive Health Care, 35(1), 9-14.

French RS, Geary R, Jones KG, Glasier A, Mercer CH, Datta J et al. (2017). Where do women and men in Britain obtain contraception? Findings from the third National Survey of Sexual Attitudes and Lifestyles (Natsal-3). Journal of Family Planning and Reproductive Health Care, [Submitted for publication].

Fuentes EC \& Azize-Vargas Y (2009). Knowledge, attitudes and practices in a group of pharmacists in Puerto Rico regarding emergency contraception. Puerto Rico Health Sciences Journal, 26(3), 191-197.

Gaffaney M, Secor-Turner M, Stamm C, Borgelt L, Mimi Chau V, Topp D et al. (2016). Outdated counseling in the pharmacy? A qualitative analysis of emergency contraception access in Wyoming. Proceedings from The 30th Annual Clinical Research Meeting of the North American Society of Pediatric and Adolescent Gynecology, Toronto.

Gainer E, Blum J, Toverud EL, Portugal N, Tyden T, Nesheim BI et al. (2003). Bringing emergency contraception over the counter: experiences of nonprescription users in France, Norway, Sweden and Portugal. Contraception, 68(2), 117-124.

Gale A \& Watson MC (2011). The provision of current and future sexual health services from community pharmacies in Grampian, Scotland. International Journal of Clinical Pharmacy, 33(2), 183-190.

Gardner JS, Downing DF, Blough D, Miller L, Le S \& Shotorbani S (2008). Pharmacist prescribing of hormonal contraceptives: results of the Direct Access study. Journal of the American Pharmacists Association, 48(2), 212-226.

Gardner JS, Hutchings J, Fuller TS \& Downing D (2001). Increasing access to emergency contraception through community pharmacies: lessons from Washington State. Family Planning Perspectives, 33(4), 172-175.

Gazmararian JA, Adams MM, Saltzman LE, Johnson CH, Bruce FC, Marks JS et al. (1995). The relationship between pregnancy intendedness and physical violence in mothers of newborns. Obstetrics \& Gynecology, 85(6), 1031-1038. 
Gilliam M, Knight S \& McCarthy M (2004). Success with oral contraceptives: a pilot study. Contraception, 69(5), 413-418.

Gipson JD, Koenig MA \& Hindin MJ (2008). The effects of unintended pregnancy on infant, child, and parental health: a review of the literature. Studies in Family Planning, 39(1), 18-38.

Glasier A, Manners R, Loudon JC \& Muir A (2010). Community pharmacists providing emergency contraception give little advice about future contraceptive use: a mystery shopper study. Contraception, 82(6), 538-542.

Glasier A, Scorer J \& Bigrigg A (2008). Attitudes of women in Scotland to contraception: a qualitative study to explore the acceptability of long-acting methods. Journal of Family Planning and Reproductive Health Care, 34(4), 213-217.

Glazer AB, Wolf A \& Gorby N (2011). Postpartum contraception: needs vs. reality. Contraception, 83(3), 238-241.

Goldthwaite LM \& Shaw KA (2015). Immediate postpartum provision of longacting reversible contraception. Current Opinion in Obstetrics and Gynecology, 27(6), 460-464.

Gonsalves L \& Hindin MJ (2016). Pharmacy provision of sexual and reproductive health commodities to young people: A systematic literature review and synthesis of the evidence. Contraception, 95(4), 339-363.

Goodman S, Hendlish SK, Reeves MF \& Foster-Rosales A (2008). Impact of immediate postabortal insertion of intrauterine contraception on repeat abortion. Contraception, 78(2), 143-148.

Greene D, Sharon F, Landau C, Monastersky N, Chung F, Kim N et al. (2006). Pharmacy access to emergency contraception in California. Perspectives on Sexual and Reproductive Health, 38(1), 46-52.

Griggs SK \& Brown CM (2007). Texas community pharmacists' willingness to participate in pharmacist-initiated emergency contraception. Journal of the American Pharmacists Association, 47(1), 48-57.

Grimes DA, Lopez LM, Schulz KF, Van Vliet HAAM \& Stanwood NL (2010). Immediate post-partum insertion of intrauterine devices. Cochrane Database of Systematic Reviews, Issue 1, CD003036.

Grindlay K, Burns B \& Grossman D (2013). Prescription requirements and over-thecounter access to oral contraceptives: a global review. Contraception, 88(1), 91-96.

Grindlay K, Foster DG \& Grossman D (2014). Attitudes toward over-the-counter access to oral contraceptives among a sample of abortion clients in the United States. Perspectives on Sexual and Reproductive Health, 46(2), 83-89.

Grol R \& Wensing M (2004). What drives change? Barriers to and incentives for achieving evidence-based practice. Medical Journal of Australia, 180(6 Suppl), S5760 .

Gross T, Lafortune J \& Low C (2014). What happens the morning after? The costs and benefits of expanding access to emergency contraception. Journal of Policy Analysis and Management, 33(1), 70-93. 
Grossman D (2008). Should the contraceptive pill be available without prescription? Yes. British Medical Journal, 337, a3044.

Grossman D (2011). Over-the-counter access to oral contraceptives. Expert Review of Obstetrics \& Gynecology, 6(5), 501-508.

Grossman D, Fernandez L, Hopkins K, Amastae J, Garcia SG \& Potter JE (2008).

Accuracy of self-screening for contraindications to combined oral contraceptive use. Obstetrics \& Gynecology, 112(3), 572-578.

Grossman D, Grindlay K, Li R, Potter JE, Trussell J \& Blanchard K (2013). Interest in over-the-counter access to oral contraceptives among women in the United States. Contraception, 88(4), 544-552.

Hametz I, Hodgkiss F \& Sheehy C. (2011) Review of the community pharmacy public health service for smoking cessation and emergency hormonal contraception. The Scottish Government, Edinburgh.

Hardy E, Santos LC, Osis MJ, Carvalho G, Cecatti JG \& Fau A (1998).

Contraceptive use and pregnancy before and after introducing lactational amenorrhea (LAM) in a postpartum program. Advances in Contraception, 14(1), 59-68.

Hariparsad N (2001). Attitudes and practices of pharmacists towards emergency contraception in Durban, South Africa. The European Journal of Contraception \& Reproductive Health Care, 6(2), 87-92.

Harney C, Dude A \& Haider S (2017). Factors associated with short interpregnancy interval in women who plan postpartum LARC: a retrospective study. Contraception, 95(3), 245-250.

Hayes M, Hutchings J \& Hayes P (2000). Reducing unintended pregnancy by increasing access to emergency contraceptive pills. Maternal and Child Health Journal, 4(3), 203-208.

Heinemann K, Reed S, Moehner S \& Minh TD (2015). Risk of uterine perforation with levonorgestrel-releasing and copper intrauterine devices in the European Active Surveillance Study on Intrauterine Devices. Contraception, 91(4), 274-279.

Heller R \& Cameron ST (2016). Evaluating the attractiveness of the availability of injectable progestogen contraceptives at the community pharmacy setting in the United Kingdom. International Journal of Pharmacy Practice, 24(4), 247-252.

Heller R, Cameron ST, Briggs R, Forson N \& Glasier A (2016). Postpartum contraception: a missed opportunity to prevent unintended pregnancy and short interpregnancy intervals. Journal of Family Planning and Reproductive Health Care, 42(2), 93-98.

Higgie S, Farris K, Barber J, Kusunoki Y, Batra P, Gatny H et al. (2014). Examining young women's negative experiences in community pharmacies with contraceptives: a cross-sectional study. Proceedings from The Royal Pharmaceutical Society Annual Conference, Birmingham, UK.

Higgins SJ \& Hattingh HL (2013). Requests for emergency contraception in community pharmacy: An evaluation of services provided to mystery patients. Research in Social and Administrative Pharmacy, 9(1), 114-119. 
Hind C, Peterkin G, Downie G, Michie C \& Chisolm E (2004). Successful provision of influenza vaccine from a community pharmacy in Aberdeen. Pharmaceutical Journal, 273, 194-196.

Hind C \& Downie G (2006). Vaccine administration in pharmacies - - a Scottish success story. Pharmaceutical Journal, 277, 134-136.

Hobbs M, Taft AJ \& Amir LH (2009). The emergency contraceptive pill rescheduled: a focus group study of women's knowledge, attitudes and experiences. Journal of Family Planning and Reproductive Health Care, 35(2), 87-91.

Hopkins K, Grossman D, White K, Amastae J \& Potter JE (2012). Reproductive health preventive screening among clinic vs. over-the-counter oral contraceptive users. Contraception, 86(4), 376-382.

Hussainy SY, Stewart K, Chapman CB, Taft AJ, Amir LH, Hobbs MK et al. (2011). Provision of the emergency contraceptive pill without prescription: attitudes and practices of pharmacists in Australia. Contraception, 83(2), 159-166.

Hussainy SY, Stewart K \& Pham M-P (2015). A mystery caller evaluation of emergency contraception supply practices in community pharmacies in Victoria, Australia. Australian Journal of Primary Health, 21(3), 310-316.

International Consortium for Emergency Contraception. (2017). Emergency Contraception: status and availability database. Retrieved 12th January, 2017, from http://www.cecinfo.org/country-by-country-information/status-availability-database/.

Jackson E \& Glasier A (2011). Return of ovulation and menses in postpartum nonlactating women: a systematic review. Obstetrics \& Gynecology, 117(3), 657662.

Jarvis S (2008). Should the contraceptive pill be available without prescription? No. British Medical Journal, 337, a3056.

Jones RK, Darroch JE \& Henshaw SK (2002). Contraceptive use among US women having abortions in 2000-2001. Perspectives on Sexual and Reproductive Health, 34(6), 294-303.

Kapp N \& Curtis KM (2009). Intrauterine device insertion during the postpartum period: a systematic review. Contraception, 80(4), 327-336.

Karpilow QC \& Thomas AT (2017). Reassessing the importance of long-acting contraception. American Journal of Obstetrics and Gynecology, 216(2), 148.e1148.e14.

Kaunitz AM, Peipert JF \& Grimes DA (2014). Injectable contraception: issues and opportunities. Contraception, 89(5), 331-334.

Kavanaugh ML, Jerman J, Ethier K \& Moskosky S (2013). Meeting the contraceptive needs of teens and young adults: youth-friendly and long-acting reversible contraceptive services in US family planning facilities. Journal of Adolescent Health, 52(3), 284-292.

Keith B, Wood S, Tifft S \& Hutchings J (2014). Home-based administration of Sayana Press: review and assessment of needs in low-resource settings. Contraception, 89(5), 344-351. 
M K, E M, DK F \& E G (2004). Emergency contraception. Has over the counter availability reduced attendances at emergency departments? Emergency Medicine Journal, 21(1), 67-68.

Killick SR \& Irving G (2004). A national study examining the effect of making emergency hormonal contraception available without prescription. Human Reproduction, 19(3), 553-557.

Kim CR, Fønhus MS \& Ganatra B (2017). Self-administration of injectable contraceptives: a systematic review. British Journal of Obstetrics and Gynaecology, 124(2), 200-208.

Kramer KT. (2017). State Legislation focuses on contraceptive access. Retrieved 1st April, 2017, from http://www.astho.org/StatePublicHealth/State-LegislationFocuses-on-Contraceptive-Access/3-9-17/.

Krska J \& Morecroft CW (2010). Views of the general public on the role of pharmacy in public health. Journal of Pharmaceutical Health Services Research, 1(1), 33-38.

Kulier R, Cheng L, Fekih A, Hofmeyr GJ \& Campana A (2001). Surgical methods for first trimester termination of pregnancy. Cochrane Database of Systematic Reviews, Issue 1, CD002900.

Kulier R, Kapp N, Gülmezoglu AM, Hofmeyr GJ, Cheng L \& Campana A (2011). Medical methods for first trimester abortion. Cochrane Database of Systematic Reviews, Issue 4, CD002855.

Lader D. (2009) Opinions Survey Report No. 41. Contraception and Sexual Health 2008/09. Office for National Statistics, London.

Lakha F \& Glasier A (2006). Unintended pregnancy and use of emergency contraception among a large cohort of women attending for antenatal care or abortion in Scotland. The Lancet, 368(9549), 1782-1787.

Laliberté MC, Perreault S, Damestoy N \& Lalonde L (2012). Ideal and actual involvement of community pharmacists in health promotion and prevention: a crosssectional study in Quebec, Canada. BMC Public Health, 12, 192.

Lambeth Lewisham and Southwark Health Action Zone. (2002) A Timely Service: A Lambeth, Southwark \& Lewisham Health Action Zone project on access to emergency hormonal contraception via accredited community pharmacies. Lambeth, Southwark and Lewisham Primary Care Trusts, London.

Lancaster GA, Dodd S \& Williamson PR (2004). Design and analysis of pilot studies: recommendations for good practice. Journal of Evaluation in Clinical Practice, 10(2), 307-312.

Landau SC, Tapias MP \& McGhee BT (2006). Birth control within reach: a national survey on women's attitudes toward and interest in pharmacy access to hormonal contraception. Contraception, 74(6), 463-470.

Landau S, Besinque K, Chung F, Dries-Daffner I, Maderes NM, McGhee BT et al. (2009). Pharmacist interest in and attitudes toward direct pharmacy access to 
hormonal contraception in the United States. Journal of the American Pharmacists Association, 49(1), 43-50.

Lara R, Sánchez RA \& Aznar R (1989). Application of intrauterine device through the incision of the cesarean section [abstract only: article in Spanish]. Ginecologia $Y$ Obstetricia De Mexico, 57, 23-27.

Larsson M, Aneblom G, Odlind V \& Tydén T (2002). Reasons for pregnancy termination, contraceptive habits and contraceptive failure among Swedish women requesting an early pregnancy termination. Acta Obstetricia et Gynecologica Scandinavica, 81(1), 64-71.

Larsson M, Eurenius K, Westerling R \& Tydén T (2004). Emergency contraceptive pills over-the-counter: a population-based survey of young Swedish women. Contraception, 69(4), 309-315.

Lebetkin E, Orr T, Dzasi K, Keyes E, Shelus V, Mensah S et al. (2014). Injectable contraceptive sales at licensed chemical seller shops in Ghana: access and reported use in rural and periurban communities. International Perspectives on Sexual and Reproductive Health, 40(1), 21-27.

Lee JT, Tsai JL, Tsou TS \& Chen MC (2011). Effectiveness of a theory-based postpartum sexual health education program on women's contraceptive use: a randomized controlled trial. Contraception, 84(1), 48-56.

Leeman L (2007). Medical barriers to effective contraception. Obstetrics and Gynecology Clinics of North America, 34(1), 19-29.

Legare K, Bakshi S, Keyhani S \& Howell EA (2012). Availability of over-thecounter emergency contraception in 2 disparate New York City neighborhoods. American Journal of Public Health, 102(11), e45-e47.

Lester F, Kakaire O, Byamugisha J, Averbach S, Fortin J, Maurer R et al. (2015). Intracesarean insertion of the Copper T380A versus 6 weeks postcesarean: a randomized clinical trial. Contraception, 91(3), 198-203.

Levi E, Canitllo E, Ades V, Banks E \& Murthy A (2012). Immediate postplacental IUD insertion at cesarean delivery: a prospective cohort study. Contraception, 86(2), 102-105.

Levi EE, Stuart GS, Zerden ML, Garrett JM \& Bryant AG (2015). Intrauterine device placement during cesarean delivery and continued use 6 months postpartum: a randomized controlled trial. Obstetrics \& Gynecology, 126(1), 5-11.

Lewington G \& Marshall K (2006). Access to emergency hormonal contraception from community pharmacies and family planning clinics. British Journal of Clinical Pharmacology, 61(5), 605-608.

Lewis LN, Doherty DA, Hickey M \& Skinner SR (2010). Implanon as a contraceptive choice for teenage mothers: a comparison of contraceptive choices, acceptability and repeat pregnancy. Contraception, 81(5), 421-426.

Liambila W, Obare F \& Keesbury J (2010). Can private pharmacy providers offer comprehensive reproductive health services to users of emergency contraceptives? Evidence from Nairobi, Kenya. Patient Education and Counseling, 81(3), 368-373. 
Liu BH, Zhang LY \& Zhang BR (1983). Intrauterine contraceptive device insertion with suture fixation at cesarean section. Chinese Medical Journal, 96(2), 141-144.

Lloyd K \& Gale E (2005). Provision of emergency hormonal contraception through community pharmacies in a rural area. Journal of Family Planning and Reproductive Health Care, 31(4), 297-300.

Lopez LM, Hiller JE, Grimes DA \& Chen M (2012). Education for contraceptive use by women after childbirth. Cochrane Database of Systematic Reviews, Issue 1, CD001863.

Lopez LM, Bernholc A, Hubacher D, Stuart G \& Van Vliet HA (2015). Immediate postpartum insertion of intrauterine device for contraception. Cochrane Database of Systematic Reviews, Issue 2, CD003036.

Lunniss H, Cameron S \& Chen ZE (2016). Views of general practitioners on providing contraceptive advice and long-acting reversible contraception at the 6week postnatal visit: a qualitative study. Journal of Family Planning and Reproductive Health Care, 42(2), 99-106.

Mackie CM, Elliot L, Thomson DAM, Bigrigg A \& McAllister KF (2002). Public and private collaboration in establishing a young person's sexual health clinic in a commercial setting. Journal of Family Planning and Reproductive Health Care, 28(4), 201-203.

Mackin ML \& Clark K (2011). Emergency contraception in Iowa pharmacies before and after over the counter approval. Public Health Nursing, 28(4), 317-324.

Madden JM, Quick JD, Ross-Degnan D \& Kafle KK (1997). Undercover careseekers: simulated clients in the study of health provider behavior in developing countries. Social Science \& Medicine, 45(10), 1465-1482.

Maderas NM, Landau SC \& Taylor-McGhee B (2008). The continuum of care: a case for pharmacists as key members of the reproductive health care team. Contraception, 77(3), 139-142.

Manski R \& Kottke M (2015). A survey of teenagers'” attitudes toward moving oral contraceptives over the counter. Perspectives on Sexual and Reproductive Health, 47(3), 123-129.

Marston C, Meltzer H \& Majeed A (2005). Impact on contraceptive practice of making emergency hormonal contraception available over the counter in Great Britain: repeated cross sectional surveys. British Medical Journal, 331(7511), 271.

McAllister KF, Elliott L, Thomson DA, Bigrigg A \& Mackie CM (2002). Evaluation of a young person's sexual health service in a commercial setting. Journal of Family Planning and Reproductive Health Care, 28(4), 203-206.

McAree DP \& Scott EM (2004). Advice provision for women: community pharmacists' attitudes and skills. International Journal of Pharmacy Practice, 12(1), $37-42$.

McCance K \& Cameron S (2014). Midwives' experiences and views of giving postpartum contraceptive advice and providing long-acting reversible contraception: 
a qualitative study. Journal of Family Planning and Reproductive Health Care, 40(3), 177-183.

McIntosh J, Tsikitas L \& Dennis A (2012). Low-income women's access to contraception after health care reform in Massachusetts. Journal of the American Pharmacists Association, 52(3), 349-357.

McLoone P \& Boddy FA (1994). Deprivation and mortality in Scotland, 1981 and 1991. British Medical Journal, 309, 1465.

Michie L, Cameron ST, Glasier A, Chen ZE, Milne D \& Wilson S (2016). Provision of contraception after emergency contraception from the pharmacy: evaluating the acceptability of pharmacy for providing sexual and reproductive health services. Public Health, 135, 97-103.

Michie L, Cameron ST, Glasier A, Larke N, Muir A \& Lorimer A (2014). Pharmacybased interventions for initiating effective contraception following the use of emergency contraception: a pilot study. Contraception, 90(4), 447-453.

Middleton AJ, Naish J \& Singer N (2011). General practitioners' views on the use of the levonorgestrel-releasing intrauterine system in young, nulligravid women, in London, UK. The European Journal of Contraception \& Reproductive Health Care, 16(4), 311-318.

Miller C, Rafie S, T. W \& Cohen Landau S (2016). Teen views on access to birth control in pharmacies: a qualitative study. Proceedings from The American Pharmacists Association Conference, Baltimore, USA.

Mohamed SS-E, Mahmoud AA \& Ali AA (2013). Involvement of Sudanese community pharmacists in public health activities. International Journal of Clinical Pharmacy, 35(3), 393-400.

Mohllajee AP, Curtis KM, Morrow B \& Marchbanks PA (2007). Pregnancy intention and its relationship to birth and maternal outcomes. Obstetrics \& Gynecology, 109(3), 678-686.

Monastersky MNJ \& Landau SC (2006). Pharmacy and clinic partnerships to expand access to injectable contraception. Journal of the American Pharmacists Association, 47(4), 527-531.

Moreau C, Bajos N \& Trussell J (2006a). The impact of pharmacy access to emergency contraceptive pills in France. Contraception, 73(6), 602-608.

Moreau C, Cleland K \& Trussell J (2007). Contraceptive discontinuation attributed to method dissatisfaction in the United States. Contraception, 76(4), 267-272.

Moreau C, Bouyer J, Goulard H \& Bajos N (2005). The remaining barriers to the use of emergency contraception: perception of pregnancy risk by women undergoing induced abortions. Contraception, 71(3), 202-207.

Moreau C, Trussell J \& Bajos N (2006b). The determinants and circumstances of use of emergency contraceptive pills in France in the context of direct pharmacy access. Contraception, 74(6), 476-482. 
Murphy C \& British Pregnancy Advisory Service. (2014) Sex and contraception after childbirth: supporting women's choices in the postnatal period. British Pregnancy Advisory Service, London.

Mwalwanda CS \& Black KI (2013). Immediate post-partum initiation of intrauterine contraception and implants: a review of the safety and guidelines for use. Australian and New Zealand Journal of Obstetrics and Gynaecology, 53(4), 331-337.

National Institute for Health and Care Excellence (NICE). (2006). Postnatal care: Routine postnatal care of women and their babies [CG37]. Retrieved 23rd February, 2017, from http://www.nice.org.uk/guidance/CG37.

National Institute for Health and Care Excellence (NICE). (2015). Patient Group Directions. Retrieved Friday 4th November, 2016, from https://www.nice.org.uk/guidance/mpg2.

National Institute for Health and Care Excellence (NICE). (2016). Contraception [QS129]. Retrieved 19th January, 2017, from https://www.nice.org.uk/guidance/qs129/chapter/introduction.

National Institute for Health and Care Excellence (NICE). (2017). Guidance and advice list. Retrieved 13th March, 2017, from https://www.nice.org.uk/guidance/indevelopment.

Nelson AL, Chen S \& Eden R (2009). Intraoperative placement of the Copper T-380 intrauterine devices in women undergoing elective cesarean delivery: a pilot study. Contraception, 80(1), 81-83.

Nelson AL \& Jaime CM (2009). Accuracy of information given by Los Angeles County pharmacies about emergency contraceptives to sham patient in need. Contraception, 79(3), 206-210.

Nettleman MD, Chung H, Brewer J, Ayoola A \& Reed PL (2007). Reasons for unprotected intercourse: analysis of the PRAMS survey. Contraception, 75(5), 361366.

Newton J, Harper M \& Chan K (1977). Immediate post-placental insertion of intrauterine contraceptive devices. The Lancet, 310(8032), 272-274.

NHS Choices Website. (2015). Where can I get contraception? Retrieved 16th March, 2017, from http://www.nhs.uk/Conditions/contraception-guide/Pages/wherecan-i-get-contraception.aspx.

NHS Scotland Information Services Division. (2010). Deprivation. Retrieved October 9th, 2014, from http://www.isdscotland.org/Products-and-Services/GPDSupport/Deprivation.

NHS Scotland Information Services Division. (2014a). Abortion Statistics 2013. http://www.isdscotland.org/Health-Topics/Sexual-Health/Publications/.

NHS Scotland Information Services Division. (2014b). Births in Scottish Hospitals: year ending 31 st March 2013. Retrieved 1st September, 2014, from http://www.isdscotland.org/Health-Topics/Maternity-and-births/Publications/. 
NHS Scotland Information Services Division. (2016a). Abortion Statistics 2015. Retrieved 12th March, 2017, from http://www.isdscotland.org/Health-Topics/SexualHealth/Publications/.

NHS Scotland Information Services Division. (2016b). Long acting reversible methods of contraception in Scotland. Retrieved 3rd February, 2017, from http://www.isdscotland.org/Health-Topics/Sexual-Health/Publications/.

Norman WV, Soon JA, Panagiotoglou D, Albert A \& Zed PJ (2015). The acceptability of contraception task-sharing among pharmacists in Canada - the ACT-Pharm study. Contraception, 92(1), 55-61.

Ogburn JA, Espey E \& Stonehocker J (2005). Barriers to intrauterine device insertion in postpartum women. Contraception, 72(6), 426-429.

Orr KK \& Kachur SG (2007). Community pharmacists"' opinions regarding emergency contraception: a survey in Rhode Island. Journal of the American Pharmacists Association, 47(4), 504-507.

Orrell C, Leong A, Boyd M \& Boardman H (2015). An evaluation of pharmacy customer views of services that should be available from community pharmacy. Proceedings from The Royal Pharmaceutical Society Annual Conference, Birmingham, UK.

Paluck EC, Stratton TP \& Eni GO (1994). Community pharmacists' participation in health education and disease prevention activities. Canadian Journal of Public Health, 85(6), 389-392.

Parsons J, Adams C, Aziz N, Holmes J, Jawad R \& Whittlesea C (2013). Evaluation of a community pharmacy delivered oral contraception service. Journal of Family Planning and Reproductive Health Care, 39(2), 97-101.

Paul C, Skegg DCG \& Williams S (1997). Depot medroxyprogesterone acetate. Patterns of use and reasons for discontinuation. Contraception, 56(4), 209-214.

Pharmaceutical Journal News Team. (2011). New specialist contraception service commissioned in Newcastle. Retrieved 19th March, 2015, from http://www.pharmaceutical-journal.com/news-and-analysis/news/new-specialistcontraception-service-commissioned-in-newcastle/11083246.article.

Picardo C \& Ferreri S (2010). Pharmacist-administered subcutaneous depot medroxyprogesterone acetate: a pilot randomized controlled trial. Contraception, 82(2), 160-167.

Polis CB, Grimes DA, Schaffer K, Blanchard K, Glasier A \& Harper C (2007). Advance provision of emergency contraception for pregnancy prevention. Cochrane Database of Systematic Reviews, Issue 2, CD005497.

Polis CB \& Zabin LS (2012). Missed conceptions or misconceptions: perceived infertility among unmarried young adults in the United States. Perspectives on Sexual and Reproductive Health, 44(1), 30-38.

Potter JE, McKinnon S, Hopkins K, Amastae J, Shedlin MG, Powers DA et al. (2011). Continuation of prescribed compared with over-the-counter oral contraceptives. Obstetrics \& Gynecology, 117(3), 551-557. 
Potter JE, Hubert C, Stevenson AJ, Hopkins K, Aiken ARA, White K et al. (2016). Barriers to postpartum contraception in Texas and pregnancy within 2 years of delivery. Obstetrics \& Gynecology, 127(2), 289-296.

Potter JE, White K, Hopkins K, Amastae J \& Grossman D (2010). Clinic versus over-the-counter access to oral contraception: choices women make along the USMexico Border. American Journal of Public Health, 100(6), 1130-1136.

Potter LS, Dalberth BT, Cañamar R \& Betz M (1997). Depot medroxyprogesterone acetate pioneers: a retrospective study at a North Carolina health department. Contraception, 56(5), 305-312.

Prabhakaran S \& Sweet A (2012). Self-administration of subcutaneous depot medroxyprogesterone acetate for contraception: feasibility and acceptability. Contraception, 85(5), 453-457.

Prager SW \& McCoy EE (2015). Immediate postpartum intrauterine contraception insertion. Obstetrics and Gynecology Clinics of North America, 42(4), 569-582.

(2004). Primary Medical Services (Scotland) Act. Retrieved 17th March, 2017, from http://www.legislation.gov.uk/asp/2004/1/part/1.

Puzey M (2005). Mirena at caesarean section. European Journal of Contraception \& Reproductive Healthcare, 10(3), 164-167.

Queddeng K, Chaar B \& Williams K (2011). Emergency contraception in Australian community pharmacies: a simulated patient study. Contraception, 83(2), 176-182.

Quilliam S (2013). "I honestly didn't know that I could": the rise and rise of inpharmacy sexual and reproductive health guidance. Journal of Family Planning and Reproductive Health Care, 40(1), 66-68.

Rafie S \& El-Ibiary SY (2011). Student pharmacist perspectives on providing pharmacy-access hormonal contraception services. Journal of the American Pharmacists Association, 51(6), 762-765.

Rafie S, Haycock M, Rafie S, Yen S \& Harper CC (2012). Direct pharmacy access to hormonal contraception: California physician and advanced practice clinician views. Contraception, 86(6), 687-693.

Rafie S, Kelly S, Gray EK, Wong M, Gibbs S \& Harper CC (2015). Provider opinions regarding expanding access to hormonal contraception in pharmacies. Women's Health Issues.

Rafie S, McIntosh J, Shealy KM, Borgelt LM, Forinash A, Shrader SP et al. (2014). Roles of the pharmacist in the use of safe and highly effective long-acting reversible contraception: an opinion of the women's health practice and research network of the American College of Clinical Pharmacy. Pharmacotherapy, 34(9), 991-999.

Rafie S \& El-Ibiary SY (2014). California pharmacy student perceptions of confidence and curricular education to provide direct pharmacy access to hormonal contraception. Pharmacy Education, 14(1), 31-36.

Ragland D, Payakachat N \& Stafford RA (2015). Emergency contraception counseling in a retail pharmacy setting: a pilot study. Journal of Pharmacy Practice, 28(3), 261-265. 
Raine R, Walt G \& Basnett I (2004). The White Paper on public health. British Medical Journal, 329(7477), 1247-1248.

Raine TR, Harper CC, Rocca CH, Fischer R, Padian N, Klausner JD et al. (2005). Direct access to emergency contraception through pharmacies and effect on unintended pregnancy and STIs: a randomized controlled trial. Journal of the American Medical Association, 293(1), 54-62.

Rani R (2008). Pharmacy-based sexual health services and clinical governance. Journal of Family Planning and Reproductive Health Care, 34(4), 274.

Rasch V (2002). Contraceptive failure: results from a study conducted among women with accepted and unaccepted pregnancies in Denmark. Contraception, 66(2), 109-116.

Ratanajamit C \& Chongsuvivatwong V (2001). Survey of knowledge and practice on oral contraceptive and emergency contraceptive pills of drugstore personnel in Hat Yai, Thailand. Pharmacoepidemiology and drug safety, 10(2), 149-156.

Ratanajamit C, Chongsuvivatwong V \& Geater AF (2002). A randomized controlled educational intervention on emergency contraception among drugstore personnel in southern Thailand. Journal of the American Medical Women's Association, 57(4), 196-199.

Raymond EG, Stewart F, Weaver M, Monteith C \& Van Der Pol B (2006). Impact of increased access to emergency contraceptive pills: a randomized controlled trial. Obstetrics \& Gynecology, 108(5), 1098-1106.

Raymond EG, Trussell J \& Polis CB (2007). Population effect of increased access to emergency contraceptive pills: a systematic review. Obstetrics \& Gynecology, 109(1), 181-188.

Richard CAH, Kinzey H \& Masters K (2015). Pharmacists' attitudes towards three models of expanded access to hormonal contraception in pharmacies. International Journal of Medicine and Pharmacy, 3(1), 5-16.

Richards E, Rafie S, Cohen Landau S \& Wilkinson T (2015). Pharmacists' views on California's new law for pharmacy access to contraception. Proceedings from The North American Forum on Family Planning, Chicago, USA.

Rocca CH, Schwarz EB, Stewart FH, Darney PD, Raine TR \& Harper CC (2007). Beyond access: acceptability, use and nonuse of emergency contraception among young women. American Journal of Obstetrics and Gynecology, 196(1), 29. e1-6.

Rose SB \& Lawton BA (2012). Impact of long-acting reversible contraception on return for repeat abortion. American Journal of Obstetrics \& Gynecology, 206(1), 37.e1-6.

Rosenberg MJ \& Waugh MS (1998). Oral contraceptive discontinuation: a prospective evaluation of frequency and reasons. American Journal of Obstetrics and Gynecology, 179(3), 577-582.

Royal College of General Practitioners. (2014) RCGP Position Statement on the Commissioning of Sexual and Reproductive Healthcare in England. RCGP, London. 
Royal College of Obstetricians and Gynaecologists. (2011) The Care of Women Requesting Induced Abortion. Evidence-Based Clinical Guideline Number 7. RCOG, London.

Royal College of Obstetricians and Gynaecologists. (2015) Best Practice in Postpartum Family Planning. Royal College of Obstetricians and Gynaecologists, London.

Rubin AG, Gold MA, Kim Y \& Schwarz EB (2011). Use of emergency contraception by US teens: effect of access on promptness of use and satisfaction. Journal of Pediatric and Adolescent Gynecology, 24(5), 286-290.

Ruiz-velasco V, Garcia C \& Castro H (1982). Cesarean section IUD insertion. Contraceptive Delivery Systems, 3(1), 21-24.

Ryder H, Aspden T \& Sheridan J (2015). The Hawke's Bay Condom Card Scheme: a qualitative study of the views of service providers on increased, discreet access for youth to free condoms. International Journal of Pharmacy Practice, 23(6), 381-389.

Saeed GA, Fakhar S, Rahim F \& Tabassum S (2008). Change in trend of contraceptive uptake - - effect of educational leaflets and counseling. Contraception, 77(5), 377-381.

Sampson O, Navarro SK, Khan A, Hearst N, Raine TR, Gold M et al. (2009).

Barriers to adolescents' getting emergency contraception through pharmacy access in California: differences by language and region. Perspectives on Sexual and Reproductive Health, 41(2), 110-118.

Samson FD, Loren R, Downing N, Schroeppel S, Kelly PJ \& Ramaswamy M (2013). Availability of emergency contraception in rural and urban pharmacies in Kansas. The Journal of Rural Health, 29(1), 113-118.

Santelli J, Rochat R, Hatfield Timajchy K, Gilbert BC, Curtis K, Cabral R et al. (2003). The measurement and meaning of unintended pregnancy. Perspectives on Sexual and Reproductive Health, 35(2), 94-101.

Saramunee K, Krska J, Mackridge A, Richards J, Suttajit S \& Phillips-Howard P (2014). How to enhance public health service utilization in community pharmacy?: General public and health providers' perspectives. Research in Social and Administrative Pharmacy, 10(2), 272-284.

Say L, Brahmi D, Kulier R, Campana A \& Gülmezoglu AM (2002). Medical versus surgical methods for first trimester termination of pregnancy. Cochrane Database of Systematic Reviews, Issue 2, CD0003037.

Say L, Chou D, Gemmill A, Tunçalp Ö, Moller A-B, Daniels J et al. (2014). Global causes of maternal death: a WHO systematic analysis. The Lancet Global Health, 2(6), e323-e333.

Schenk KD (2003). Emergency contraception: lessons learned from the UK. Journal of Family Planning and Reproductive Health Care, 29(2), 35-40.

Schünmann C \& Glasier A (2006). Measuring pregnancy intention and its relationship with contraceptive use among women undergoing therapeutic abortion. Contraception, 73(5), 520-524. 
Sedgh G, Bearak J, Singh S, Bankole A, Popinchalk A, Ganatra B et al. (2016). Abortion incidence between 1990 and 2014: global, regional, and subregional levels and trends. The Lancet, 388(10041), 258-267.

Seston EM, Elliott RA, Noyce PR \& Payne K (2007). Women's preferences for the provision of emergency hormonal contraception services. Pharmacy World \& Science, 29(3), 183-189.

Seston EM, Holden K \& Cantrill J (2001). Emergency hormonal contraception: the community pharmacy perspective. Journal of Family Planning and Reproductive Health Care, 27(4), 203-208.

Shacter HE, Gee RE \& Long JA (2007). Variation in availability of emergency contraception in pharmacies. Contraception, 75(3), 214-217.

Shah MA, Shah NM, Al-Rahmani E, Behbehani J \& Radovanovic Z (2001). Overthe-counter use of oral contraceptives in Kuwait. International Journal of Gynecology \& Obstetrics, 73(3), 243-251.

Shah PS, Balkhair T, Ohlsson A, Beyene J, Scott F \& Frick C (2011). Intention to become pregnant and low birth weight and preterm birth: a systematic review. Maternal and Child Health Journal, 15(2), 205-216.

Shotorbani S, Miller L, Blough DK \& Gardner J (2006). Agreement between women's and providers' assessment of hormonal contraceptive risk factors. Contraception, 73(5), 501-506.

Shukla M \& Qureshi S (2012). Post-placental intrauterine device insertion - a five year experience at a tertiary care centre in north India. Indian Journal of Medical Research, 136(3), 432-435.

Sidebotham P, Heron J \& The ALSPAC Study Team (2003). Child maltreatment in the "“"children of the nineties":" the role of the child. Child Abuse \& Neglect, 27(3), 337-352.

Smith E \& McLellan A (2014). Comment on 'Midwives' experiences and views of giving postpartum contraceptive advice and long-acting reversible contraception: a qualitative study'. Journal of Family Planning and Reproductive Health Care, 40(4), 312-313.

Smith GCS, Pell JP \& Dobbie R (2003). Interpregnancy interval and risk of preterm birth and neonatal death: retrospective cohort study. British Medical Journal, 327(7419), 313.

Sommers SD, Chaiyakunapruk N, Gardner JS \& Winkler J (2001). The emergency contraception collaborative prescribing experience in Washington State. Journal of the American Pharmacists Assocation, 41(1), 60-66.

Sonalkar S \& Kapp N (2015). Intrauterine device insertion in the postpartum period: a systematic review. The European Journal of Contraception \& Reproductive Health Care, 20(1), 4-18.

Soon JA, Levine M, Osmond BL, Ensom MHH \& Fielding DW (2005). Effects of making emergency contraception available without a physician's prescription: a population-based study. Canadian Medical Association Journal, 172(7), 878-883. 
Stanback J, Lebetkin E, Orr T \& Malarcher S (2014). Sale and provision of injectable contraceptives in drug shops in developing countries: conclusions from a technical consultation. Contraception, 91(2), 93-96.

Stevens-Simon C, Kelly L \& Kulick R (2001). A village would be nice but. it takes a long-acting contraceptive to prevent repeat adolescent pregnancies. American Journal of Preventative Medicine, 21(1), 60-65.

Stevens-Simon C, Sheeder J \& Harter S (2005). Teen contraceptive decisions: childbearing intentions are the tip of the iceberg. Women \& Health, 42(1), 55-73.

Sucak A, Ozcan S, Çelen C, Çaglar T, Göksu G \& Danisman N (2015). Immediate postplacental insertion of a copper intrauterine device: a pilot study to evaluate expulsion rate by mode of delivery. BMC Pregnancy and Childbirth, 15(1), 202.

Sucato GS, Gardner JS \& Koepsell TD (2001). Adolescents' use of emergency contraception provided by Washington State pharmacists. Journal of Pediatric and Adolescent Gynecology, 14(4), 163-169.

Sweeney L-A, Molloy GJ, Byrne M, Murphy AW, Morgan K, Hughes CM et al. (2015). A qualitative study of prescription contraception use: the perspectives of users, general practitioners and pharmacists. PloS one, 10(12), e0144074.

Tan SP, Yuen MY, Boardman H \& Boyd M (2015). Evaluation of community pharmacy customers' views of the seasonal influenza vaccination and community pharmacy vaccine services. Proceedings from The Royal Pharmaceutical Society Annual Conference, Birmingham, UK.

Tang JH, Dominik RC, Zerden ML, Verbiest SB, Brody SC \& Stuart GS (2014). Effect of an educational script on postpartum contraceptive use: A randomized controlled trial. Contraception, 90(2), 162-167.

Tavares MP \& Foster AM (2016). Emergency contraception in a public health emergency: exploring pharmacy availability in Brazil. Contraception, 94(2), 109114.

Taylor B (2008). Developments in pharmacy-based sexual health services. Journal of Family Planning and Reproductive Health Care, 34(3), 143-145.

The Scottish Government. (2011) Sexual Health and Blood Borne Virus Framework 2011-15. The Scottish Government, Edinburgh.

The Scottish Government. (2012a). Scottish Index of Multiple Deprivation 2012: a National Statistics Publication for Scotland. Retrieved 3rd October, 2016, from http://www.gov.scot/Topics/Statistics/SIMD.

The Scottish Government. (2012b) Scottish Index of Multiple Deprivation 2012: a national statistics publication for Scotland. The Scottish Government, Edinburgh.

The Scottish Government. (2015) Sexual Health and Blood Borne Virus Framework 2015-2020 Update. The Scottish Government, Edinburgh.

The Scottish Government: Pharmacy \& Medicines Division. (2013) Seasonal Influenza Immunisation 2013-14: Vaccine Supply Arrangements. The Scottish Government, Edinburgh. 
Tocce K, Sheeder J, Python J \& Teal SB (2012a). Long acting reversible contraception in postpartum adolescents: early initiation of etonogestrel implant is superior to IUDs in the outpatient setting. Journal of Pediatric and Adolescent Gynecology, 25(1), 59-63.

Tocce KM, Sheeder JL \& Teal SB (2012b). Rapid repeat pregnancy in adolescents: do immediate postpartum contraceptive implants make a difference? American Journal of Obstetrics and Gynecology, 206(6), 481.e1-7.

Todd A, Copeland A, Husband A, Kasim A \& Bambra C (2015). Access all areas? An area-level analysis of accessibility to general practice and community pharmacy services in England by urbanity and social deprivation. British Medical Journal Open, 5(5), e007328.

Torres L, Turok DK, Sanders J, Clark E \& Godfrey E (2014). Focused contraceptive counseling and case management versus usual care in women postpartum from a preterm birth: a randomized-controlled trial. Contraception, 90(3), 317.

Trussell J (2011). Contraceptive failure in the United States. Contraception, 83(5), 397-404.

Trussell J, Vaughan B \& Stanford J (1999). Are all contraceptive failures unintended pregnancies? Evidence from the 1995 National Survey of Family Growth. Family Planning Perspectives, 31(5), 246-260.

Van Riper KK \& Hellerstedt WL (2005). Emergency contraceptive pills: dispensing practices, knowledge and attitudes of South Dakota pharmacists. Perspectives on Sexual and Reproductive Health, 37(1), 19-24.

Vanthuyne A, Joyce G \& Pittrof R (2014). Comment on 'Midwives' experiences and views of giving postpartum contraceptive advice and providing long-acting reversible contraception: a qualitative study. Journal of Family Planning and Reproductive Health Care, 40(4), 313-314.

Vu K, Rafie S, Grindlay K \& Grossman D (2015). Pharmacist intentions to participate in expanded pharmacy access to hormonal contraception authority in California. Proceedings from The American Pharmacists Association, San Diego, USA.

Warner JG, Portlock J, Smith J \& Rutter P (2013). Increasing seasonal influenza vaccination uptake using community pharmacies: experience from the Isle of Wight, England. International Journal of Pharmacy Practice, 21(6), 362-367.

Washington CI, Jamshidi R, Thung SF, Nayeri UA, Caughey AB \& Werner EF (2015). Timing of postpartum intratuerine device placement: a cost-effectiveness analysis. Fertility and Sterility, 103(1), 131-137.

Weinberger M, Murray MD, Marrero DG, Brewer N, Lykens M, Harris LE et al. (2002). Issues in conducting randomized controlled trials of health services research interventions in nonacademic practice settings: the case of retail pharmacies. Health Services Research, 37(4), 1067-1077.

Wellings K, Jones KG, Mercer CH, Tanton C, Clifton S, Datta J et al. (2013). The prevalence of unplanned pregnancy and associated factors in Britain: findings from 
the third National Survey of Sexual Attitudes and Lifestyles (Natsal-3). The Lancet, 382(9907), 1807-1816.

Welsh Assembly Government. (2010). Sexual Health and Wellbeing Action Plan for Wales 2010-2015. Retrieved 25/11, 2016, from

http://gov.wales/docs/phhs/publications/101110sexualhealthen.pdf.

Whitaker AK, Endres LK, Mistretta SQ \& Gilliam ML (2014). Postplacental insertion of the levonorgestrel intrauterine device after cesarean delivery vs delayed insertion: a randomized controlled trial. Contraception, 89(6), 534-539.

Wilkinson TA, Fahey N, Shields C, Suther E, Cabral HJ \& Silverstein M (2012). Pharmacy communication to adolescents and their physicians regarding access to emergency contraception. Pediatrics, 129(4), 624-629.

Wilkinson TA, Vargas G, Fahey N, Suther E \& Silverstein M (2014). ““I'll see what I can do"': What adolescents experience when requesting emergency contraception. Journal of Adolescent Health, 54(1), 14-19.

Williams CM, Larsen U \& McCloskey LA (2008). Intimate partner violence and women's contraceptive use. Violence Against Women, 14(12), 1382-1396.

Williams RL, Hensel DJ \& Fortenberry JD (2013). Self-adminstration of subcutaneous depot medroxyprogesterone acetate by adolescent women. Contraception, 88(3), 401-407.

Wilson EK, Fowler CI \& Koo HP (2013). Postpartum contraceptive use among adolescent mothers in seven states. Journal of Adolescent Health, 52(3), 278-283.

Wilson EK, Samandari G, Koo HP \& Tucker C (2011). Adolescent mothers' postpartum contraceptive use: a qualitative study. Perspectives on Sexual and Reproductive Health, 43(4), 230-237.

Winner B, Peipert JF, Zhao Q, Buckel C, Madden T, Allsworth JE et al. (2012). Effectiveness of long-acting reversible contraception. New England Journal of Medicine, 366(21), 1998-2007.

World Health Organisation (1998). Task Force on Postovulatory Methods of Fertility Regulation. Randomized controlled trial of levonorgestrel versus the Yuzpe regimen of combined oral contraceptives for emergency contraception. The Lancet, 352(9126), 428-433.

World Health Organisation. (2006) Report of a WHO Technical Consultation on Birth Spacing. World Health Organisation, Geneva.

Xu JX, Connell C \& Chi I-C (1992). Immediate postpartum intrauterine device insertion: - a report on the Chinese experience. Advances in Contraception, 8(4), 281-290.

Yazd NKK, Gaffaney M, Stamm CA, Borgelt L, Gross L, McNair B et al. (2016). Physicians and pharmacists on over-the-counter contraceptive access for women. Journal of General Internal Medicine, 31(SUPPL 2), S342-S343.

Yeatman SE, Potter JE \& Grossman DA (2006). Over the counter access, changing WHO guidelines, and contraindicated oral contraceptive use in Mexico. Studies in Family Planning, 37(3), 197-204. 
Yee L \& Simon M (2011a). Urban minority women's perceptions of and preferences for postpartum contraceptive counseling. Journal of Midwifery \& Women's Health, 56(1), 54-60.

Yee LM \& Simon MA (2011b). Perceptions of coercion, discrimination and other negative experiences in postpartum contraceptive counseling for low-income minority women. Journal of Health Care for the Poor and Underserved, 22(4), 13871400 .

Zerden ML, Tang JH, Stuart GS, Norton DR, Verbiest SB \& Brody S (2015).

Barriers to receiving long-acting reversible contraception in the postpartum period. Women's Health Issues, 25(6), 616-621.

Zhou S-W \& Chi I-C (1991). Immediate postpartum IUD insertions in a Chinese hospital-: a two year follow-up. International Journal of Gynecology \& Obstetrics, $35(2), 157-164$. 


\section{Appendix 1: Questionnaires administered in research described in this thesis}

1. Questionnaire administered to patients in Chapter 3: Depo at the pharmacy

2. Questionnaire administered to patients exiting the project described in Chapter 5: Exit Questionnaire

3. Questionnaire administered to postpartum women in Chapter 6: 


\section{Depo-provera contraception at the pharmacy}

Depo-provera ('the jag') is a kind of contraception that is given every 3 months by an injection. At the moment this injection is into the muscle, and needs to be given by a doctor or nurse, which means visiting a clinic or your GP every 3 months. We now have a new kind of the jag which is an injection just under the skin, and this is the kind of injection a pharmacist can give.

We are doing a survey to find out what women think about this. Your answers are completely confidential and nobody in the clinic will know what you have ticked. We would be very grateful if you could take 5 minutes to fill in this quick questionnaire. Thank you.

\section{What contraception are you using at the moment?}

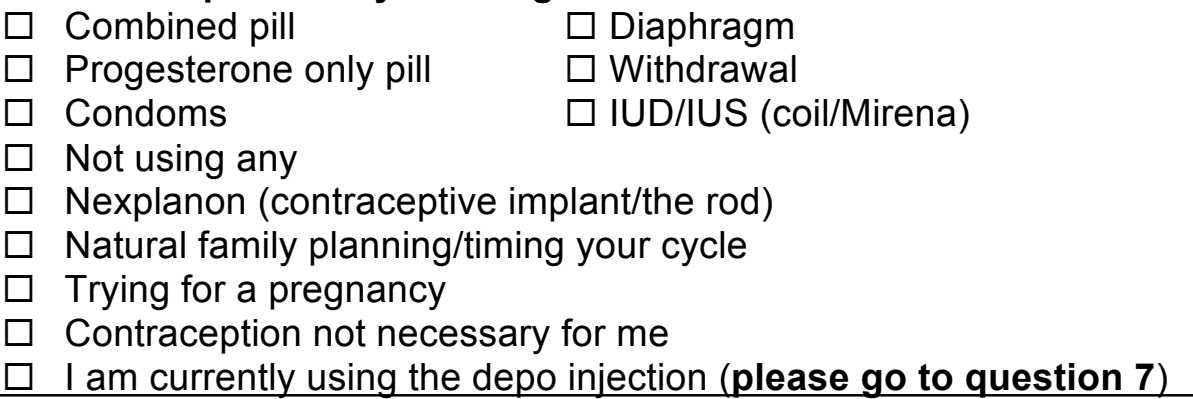

\section{Have you ever used the $\mathbf{3}$ monthly depo injection?}

\section{Yes}

\section{What was your reason for stopping it? (Please tick all that apply)}

$\square$ Having to see a doctor or nurse every 3 months

Painful injection

Weight gain

Acne

Periods stopping

Irregular bleeding

Mood swings

Headaches

Wanting to get pregnant

Wanting something longer acting

$\square$ Wanted a hormone free method

$\square$ Other (please let us know)

(Please go to question 6)
No

4. Have you ever considered using the depo injection?

$\square$ Yes (go to question 5) No (go to question 6)

5. What was your reason for deciding not to use it?

$\square$ Having to see a doctor or nurse every 3 months

$\square$ Fear of needles/injections

$\square$ Worry about weight gain

$\square$ Worry about bone health

$\square$ Don't want a method where your periods stop

$\square$ Delay in return to fertility

$\square$ Prefer something longer acting

$\square$ Other people put me off

$\square$ Other (please let us know)

(Please go to question 6) 
6. If you are NOT using depo, would you consider using it if you could get an injection under your skin given at your local pharmacy?

Yes (go to question 9)

No (go to question 10)

7. If you are a current user of the depo jag, how long have you been using it?

$\begin{array}{ll}\square & \text { I am getting my first injection today } \\ \square & \text { Less than a year } \\ \square & 1 \text { to } 3 \text { years } \\ \square & \text { More than } 3 \text { years }\end{array}$

8. If you are a current user of the depo jag, would you be interested in getting a jag under the skin at the pharmacy?

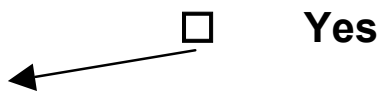

9. Why would this be a good option for you? (Please tick as many as you like)

Better opening hours at pharmacy

Easier to get there

Closer to my house

Quicker appointment

Prefer an injection under the skin to into the muscle

$\square$ Other (please let us know)
No

10. Why would this not be a good option for you? (Please tick as many as you like)

Prefer to see a nurse or doctor every 12 weeks Better opening hours here Easier to get here Prefer an injection into the muscle to under the skin Would never consider using depo

Want a hormone free method Other (please let us know)

Thank you very much for filling in this questionnaire. Please hand it back to the receptionists. We are very grateful for your help with our research. 
Thank you for participating in our research into women receiving their contraceptive injection at the pharmacy. We would be grateful if you could answer some questions now the project is over.

Name:

Why are you completing this exit questionnaire?
$\square$ The project is finished
$\square$ I left the project early

How many times did you attend the pharmacist for your contraceptive injection?
$\square 1$
$\square 3$
$\square 2$

What did you find to be the benefits of attending the pharmacy? (Tick all that apply)
$\square$ Better opening hours
$\square$ Nearer to me
$\square$ Easier to get to a pharmacy
$\square$ Flexibility of attending different pharmacists
$\square$ Shorter waiting time
$\square$ Quicker appointment
$\square$ Can do other things at the same time in the pharmacy
$\square$ Don't think there will be any real benefit
$\square$ Other (please say)

And if you had to pick one as being the best thing about it, what was it? (Pick just one)
$\square$ Better opening hours
$\square$ Nearer to me
$\square$ Easier to get to a pharmacy
$\square$ Flexibility of attending different pharmacists
$\square$ Shorter waiting time
$\square$ Quicker appointment
$\square$ Could do other things at the same time in the pharmacy
$\square$ Don't think there was any real benefit
$\square$ Other (please say)

What was the worst thing about attending the pharmacy?
$\square$ Wasn't able to discuss any problems I had with the method
$\square$ Feel more comfortable seeing a nurse or doctor than a pharmacist
$\square$ Wasn't able to discuss other medical problems
$\square$ Better opening hours at doctors/sexual health clinic
$\square$ Doctors/sexual health clinic is nearer to me
$\square$ Easier to get to GP/clinic
$\square$ There was a longer waiting time at the pharmacy
$\square$ There was a longer appointment at the pharmacy
$\square$ Can do other things at the same time in the sexual health clinic
$\square$ No downside to attending the pharmacy
$\square$ Other (please say)

If you had to pick one thing that was the worst, what would it be? (Pick just one)

$\square$ Wasn't able to discuss any problems I had with the method

$\square$ Feel more comfortable seeing a nurse or doctor than a pharmacist

$\square$ Wasn't able to discuss other medical problems 
Better opening hours at doctors/sexual health clinic

$\square$ Doctors/sexual health clinic is nearer to me

$\square$ Easier to get to GP/clinic

$\square$ There was a longer waiting time at the pharmacy

$\square$ There was a longer appointment at the pharmacy

$\square$ Can do other things at the same time in the sexual health clinic

$\square$ No downside to attending the pharmacy

$\square$ Other (please say)

If you left the project early without getting all 3 injections at the pharmacy, please can you tell us why?

Can you tell us anything else that you think we should know about what being in this research project was like? We will keep everything you say confidential from the pharmacy team.

Thank you very much for agreeing to participate in our research. 


\section{Questionnaire for postpartum women}

Q1. (a) While you were pregnant (i.e. at antenatal clinics or classes before giving birth) did any healthcare professional (doctor or nurse or midwife) discuss with you your plans for contraception after the birth of the baby? (please tick the box next to the correct response)

Yes, contraception was discussed and raised by the health care professional

$\square$ Yes, contraception was raised by me/my partner

$\square$ Yes, contraception was discussed but I can't remember who raised it

$\square$ No, contraception not discussed at all

Don't know/can't remember

(b) If yes, then how helpful was the discussion with the healthcare professional about contraception? (please tick the box next to the statement that corresponds most to how you feel)

The discussion about contraception was very helpful

The discussion about contraception was quite helpful

$\square$ The discussion about contraception was neither helpful nor unhelpful

$\square$ The discussion about contraception was not very helpful

The discussion about contraception was completely unhelpful

Q2. What type of birth did you have?

Normal vaginal birth

$\square$ Forceps OR Ventouse

$\square$ Emergency caesarean section

Elective (PLANNED) caesarean section

Q3. What method of contraception are you planning to use? (please tick appropriate box)

$\square$ Intrauterine device (IUD or copper coil)

$\square$ Injectable/Jag (Depo Provera)

$\square$ Implant (Nexplanon)

$\square$ Combined pill

$\square$ Progestogen only pill (minipill)

$\square$ Diaphragm

$\square$ Condoms

$\square$ Contraceptive patch

$\square$ Vaginal contraceptive ring

$\square$ Planning female sterilisation

$\square$ Breastfeeding as a method of

$\square$ Other - please specify: contraception

$\square$ Planning male vasectomy

$\square$ Don't know/not sure

$\square$ Mirena (IUS or hormone coil) 
Q4. Do you think that any of the following things might make it difficult for you to obtain contraception? (please tick all boxes that apply)

$\square$ Finding time for the extra visit to get contraception

$\square$ Getting organised with a young baby to care for

$\square$ Getting transportation to the clinic for contraception

$\square$ Getting childcare for me to attend another visit

$\square$ Taking time to recover from childbirth/and or c-section

$\square$ Other-please specify:

None of the above

You may/may not be aware that the most effective methods of contraception are the intrauterine methods the IUS and IUD (can last for 5 years) and the implant (that lasts for 3 years) and are completely reversible.

Also you may/may not be aware that the IUS and IUD can be inserted at the time of the birth, thus avoiding any further need for another vaginal examination and procedure a few weeks later. Also, the implant can be inserted into the arm before you go home.

Then I would ask ALL women the next questions

Q5. (a) If you could have an intrauterine method (IUS or IUD) inserted immediately after passing the placenta (after birth) in the labour ward, or, if you have a caesarean section, at the time of the operation how likely would you be to choose this (please tick box next to statement that corresponds most to how you feel)

$\square \quad$ Very likely $\quad \square \quad$ Quite likely $\quad \square \quad$ Neither/nor

$\square \quad$ Quite unlikely $\quad \square \quad$ Very unlikely

(b) If you could choose to have the implant inserted into your arm before you go home how likely would you be to choose this (please tick box next to statement that corresponds most to how you feel)

$\square$ Very likely $\quad \square \quad$ Quite likely $\quad \square \quad$ Neither/nor

$\square \quad$ Quite unlikely $\quad \square \quad$ Very unlikely 
Q7. Have you ever given birth before? (please tick appropriate box)

$\square \quad$ Yes What year was the last birth?
$\square \quad$ No

Q8. What are your future plans regarding pregnancy? (please tick appropriate box)

$\square \quad$ I intend to try for another baby in the next 12 months

$\square$ I intend to try for another baby but not for at least one year

$\square$ I do not want any more children

$\square$ Don't know yet

Q9. Are you planning to breast feed (please tick appropriate box)

$\begin{array}{llllll}\square & \text { Yes, fully } & \square & \text { Yes, partially } & \square & \text { No }\end{array}$

Q10. What age are you? ____ years old

Q11. What is your postcode? EH

Only give the next 3 characters (e.g. EH13 7) - This information is only used to find out what area you live in and how far you live from the hospital and family planning services.

If you have any other comments that you would like to make then please write them here:

Thank you. Your responses to this questionnaire will help us to determine if women feel any improvements in contraceptive advice and provision could be made.

Thank you for taking the time to fill in this questionnaire. 


\section{Appendix 2: Published papers resulting from research described in this thesis as published}

1. Heller R, Johnstone A, Cameron ST. The feasibility of contraceptive injections at the community pharmacy. Eur J Contracept Reprod Health Care 2017 doi:10.1080/13625187.2017.1357808 [Epub ahead of print]

2. Heller R, Johnstone A, Cameron ST. Routine provision of intrauterine contraception at elective cesarean section in a national public health service: an observational study. Acta Obstet Gynecol Scand 2017;96(9):1144-1151.

3. Heller R, Cameron ST. Evaluating the attractiveness of the availability of injectable progestogen contraceptives at the community pharmacy setting in the United Kingdom. Int J Pharm Pract 2016;24(4):247-252.

4. Heller R, Cameron S, Briggs R, Forson, N, Glasier A. Postpartum contraception: a missed opportunity to prevent unintended pregnancy and short inter-pregnancy intervals. J Fam Plan Reprod Health Care 2016;42(2)93-98. 


\section{The feasibility of contraceptive injections at the community pharmacy}

\section{R. Heller , A. Johnstone \& S. T. Cameron}

To cite this article: R. Heller , A. Johnstone \& S. T. Cameron (2017): The feasibility of contraceptive injections at the community pharmacy, The European Journal of Contraception \& Reproductive Health Care, DOI: 10.1080/13625187.2017.1357808

To link to this article: http://dx.doi.org/10.1080/13625187.2017.1357808

曲 Published online: 29 Aug 2017.

Submit your article to this journal $₫$

View related articles

View Crossmark data $₫$ 


\title{
The feasibility of contraceptive injections at the community pharmacy
}

\author{
R. Heller ${ }^{\mathrm{a}}\left(\mathbb{D}\right.$, A. Johnstone ${ }^{\mathrm{b}}$ and S. T. Cameron ${ }^{\mathrm{c}}$ \\ ${ }^{a}$ Community Sexual \& Reproductive Health, Chalmers Centre, Edinburgh, UK; ${ }^{b}$ Chalmers Centre, University of Edinburgh, Edinburgh, UK; \\ ${ }^{c}$ Chalmers Centre, Sexual \& Reproductive Health, University of Edinburgh and NHS Lothian, Edinburgh, UK
}

\begin{abstract}
Purpose: The progestogen-only intramuscular injectable Depo-Provera ${ }^{\circledR}$ (depot medroxyprogesterone acetate) is an effective contraceptive method, but users need to attend a clinic every 12-13 weeks for a repeat injection from a doctor/nurse. This limits convenience of the method and may affect continuation rates. We conducted a pilot study to examine the feasibility and acceptability of users receiving the subcutaneous form of the contraception injection from pharmacists in the community pharmacy setting.

Materials and methods: Existing users of Depo-Provera ${ }^{\circledR}$, who wished to switch to the subcutaneous preparation with the same active ingredient (Sayana Press ${ }^{\circledR}$ ) were invited to attend 1 of 11 community pharmacies for up to three repeat injections, given by a pharmacist. Evaluation consisted of (i) self-administered questionnaires of women and (ii) interviews with participating pharmacists, at study exit on their respective experiences.

Results: Global unavailability of the product during the study adversely affected recruitment and retention. 50 women were recruited. Only 48 injections were delivered at the pharmacy out of a possible 150 (34\%). About 26 participants received no injections at the pharmacy and only seven (14\%) participants received all three injections at the pharmacy. Participants reported mixed experiences, with some welcoming the intervention but others experiencing difficulty with pharmacist availability. Pharmacists were enthusiastic about this expansion of their role, and did not view their availability as a barrier to service delivery.

Conclusions: Delivery of the subcutaneous contraceptive injectable from a community pharmacy may be feasible but availability of sufficient numbers of pharmacists trained in this technique is necessary for a robust model of service delivery.
\end{abstract}

\section{ARTICLE HISTORY}

Received 4 April 2017

Revised 13 July 2017

Accepted 15 July 2017

Published online 30 August

2017

\section{KEYWORDS}

Contraception; community pharmacy; contraceptive injection; subcutaneous injection

\section{Introduction}

Community pharmacists are located within patients' communities, of particular relevance for those living in areas of deprivation or rural settings [1]. In the interest of improving access to health care and also cost savings, there has been increasing attention on the ability of the community pharmacy to deliver health care, and sexual health care has been a particular focus $[2,3]$. One possible area of sexual health care that community pharmacists could become involved in is the delivery of contraception. Two small studies in the United States have demonstrated the feasibility of community pharmacists administering the subcutaneous contraceptive injection [4,5]. However, the feasibility and acceptability of this approach in a UK setting has not been addressed, crucially in a setting where all contraception and health care delivery is free.

The contraceptive injection is a reliable method of contraception, when users are able to receive regular doses in a timely fashion [6]. The methods available for use in the United Kingdom are both depot medroxyprogesterone acetate in the intra-muscular version (150 mg DMPA-IM given every 12 weeks, Depo-Provera ${ }^{\circledR}$ ) and the more recently licensed subcutaneous version (104 mg DMPA-SC given every 13 weeks, Sayana Press ${ }^{\circledR}$ ). The subcutaneous version was developed to be as straightforward to administer as possible, with a view to self-administration, but at the time this project was conceived both versions of injectable contraception were only licensed for administration by health care professionals in the United Kingdom.

Despite its popularity, discontinuation rates of the injection are reported to be as high as $42.3 \%$ at 12 months $[7,8]$. Although the primary reason women give for discontinuation is side effects $[9,10]$, the inconvenience of having to attend a clinic regularly may be a contributory factor. Due to the regular visits needed, compared to other LARC (long acting reversible contraception) methods which last for years after insertion (three years for the subdermal implant and 5 or 10 years for intrauterine methods) or short-acting methods where patients may be provided with one year's supply at a single visit [11], using the contraceptive injection entails more visits to a clinician than any other method. Research within a specialised contraceptive service in Scotland indicated that the option to receive injections from a community pharmacy might improve uptake of this method. In a survey of women attending the contraceptive service, $31 \%$ of those respondents who identified as non-users of the injectable indicated that they would be potentially interested in using this method if they could receive injections from the community pharmacy [12].

We therefore conducted a pilot study in Edinburgh, Scotland to examine the feasibility and acceptability of 
users of the contraceptive injectable receiving repeat subcutaneous injections of DMPA-SC from pharmacists in the community pharmacy setting.

\section{Methods}

\section{Selection and description of participants}

A total of 11 pharmacies throughout Edinburgh and the surrounding area were identified as suitable for participation, based on a number of factors, including: established links with the research team, a high proportion of contraceptive injectable users in close proximity to the pharmacy and convenient location of pharmacy such as a shopping centre. Pharmacists underwent training in emergency resuscitation and anaphylaxis, as well as training in giving subcutaneous injections, with supervised injections on simulated models until they felt competent. All pharmacists were provided with a DVD demonstrating the injection technique to watch before each injection. A patient group direction, which is a document allowing certain health care professionals to administer medications to specific groups without a prescription, was created to be used in this research.

Women were recruited from a large integrated sexual and reproductive health service in the centre of Edinburgh, and from peripheral clinics staffed by this centre. All women who attended who were aged 16-45 and had been using the contraceptive injection for at least six months were given information about the study and offered participation.

\section{Technical information}

Women wanting to participate received their first DMPA-SC injection with the research team at a clinic. Participants were asked to contact their chosen participating pharmacist one week before their next injection was due to arrange an appointment to receive this. If women encountered difficulties getting a pharmacist appointment they could contact the research team and attend the clinic.

Upon completion of each injection, sent information to the research team and a payment of $£ 20$ per injection plus the cost of one Sayana Press ${ }^{\circledR}$ injection $(£ 6.90)$ was paid to the pharmacy for participation in the research.

Participants were followed up by phone call or text message after their injection was due. Data was collected about any problems encountered, and whether they were happy to attend a pharmacy for their next injection. After one year of injections comprising an initial injection at clinic followed by three injections at the pharmacy, participants were invited to attend the research team for their fifth injection and to complete a questionnaire about their experience. This was also offered to any withdrawing participants who had received at least one injection at a pharmacy. The questionnaire asked about the disadvantages and advantages of attending the community pharmacy, and contained free text boxes for participants to discuss their experience.

Each participating pharmacist was invited to participate in a recorded, semi-structured interview. Interviews were recorded, transcribed and analysed. Pharmacists discussed barriers to delivering the service, and their perspective of
Table 1. Demographics of participants

\begin{tabular}{lc}
\hline Age median (range) & $28(17-48)$ years \\
BMI (body mass index): median (range) & $26(17.9-45.7) \mathrm{kg} / \mathrm{m}^{2}$ \\
Parity & $37(74 \%)$ \\
0 & $4(8 \%)$ \\
1 & $7(14 \%)$ \\
2 & $1(2 \%)$ \\
3 & $1(2 \%)$ \\
4 & \\
Previous termination of pregnancy (TOP) & $40(80 \%)$ \\
0 & $10(20 \%)$ \\
1 & \\
SIMD (Scottish index of maternal deprivation) $[13]$ & $11(22 \%)$ \\
SMID 5 (affluent) & $10(20 \%)$ \\
4 & $11(22 \%)$ \\
3 & $15(30 \%)$ \\
2 & $3(6 \%)$ \\
1 (deprived) & \\
Months using contraceptive injection & $7(14 \%)$ \\
6 & $9(18 \%)$ \\
$7-12$ & $13(26 \%)$ \\
$13-24$ & $9(18 \%)$ \\
$25-36$ & $4(8 \%)$ \\
$37-48$ & $11(22 \%)$ \\
$>48$ &
\end{tabular}

the role of the pharmacist in sexual and reproductive health care.

Approximately three months after recruitment of the first participant, and after recruitment of participant number 25, Sayana Press ${ }^{\circledR}$, the only brand of subcutaneous contraceptive injectable, became globally unavailable, a shortage lasting five months. The impact will be examined in the discussion.

Pharmacists were approached in July 2014 and recruitment began 1 October 2014. The last patient was recruited in March 2016 and the final injection was given in November 2016.

Ethical approval was granted by the National Research Ethics Service Committee East Midlands - Leicester 01 on 15th July 2014 (REC reference 14/EM/1058).

\section{Results}

\section{Demographics}

About 78 women were approached for study participation and 50 were recruited, with an age range of 17-48. Demographics and reproductive history of participants are shown in Table 1.

\section{Participant flow}

Twenty-six participants (52\%) received no injections at the pharmacy, for a variety of reasons (see Table 2). Forty-eight injections were received at a pharmacy out of a possible 150. Figure 1 demonstrates where participants received their injections. A total of 26 participants (54\%) chose not to continue with the study after one or two injections. Three participants were lost to follow-up over the course of the study.

\section{Continuation and satisfaction}

During the period DMPA-SC was unavailable 17 participants withdrew from the research. At the end of the project there were 21 participants remaining; seven received all three injections at the pharmacy, eight received two injections at 
Table 2. Timings and reasons for patient withdrawals.

Withdrew before first injection at pharmacy due $(n=3)$

Left area

Changed contraceptive method

Unacceptable side effects DMPA-SC

Withdrew after first injection at pharmacy due/given $(n=19)$

$\begin{array}{lc} & \text { First injection received at pharmacy }(n=4) \\ \text { Left area } & 0 \\ \text { Prefer to attend clinic } & 1 \\ \text { Discontinued contraceptive injection } & 1 \\ \text { Outwith protocol due to age } & 0 \\ \text { Unacceptable side effects with DMPA-SC } & 0 \\ \text { Prefer self-injection } & 2\end{array}$

Withdrew after second injection at pharmacy due/given $(n=4)$

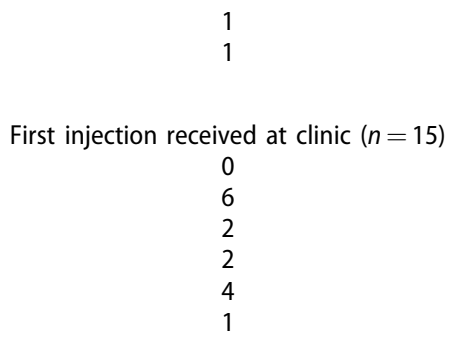

First injection received at clinic $(n=15)$

Second injection received at clinic $(n=3)$ 1 1

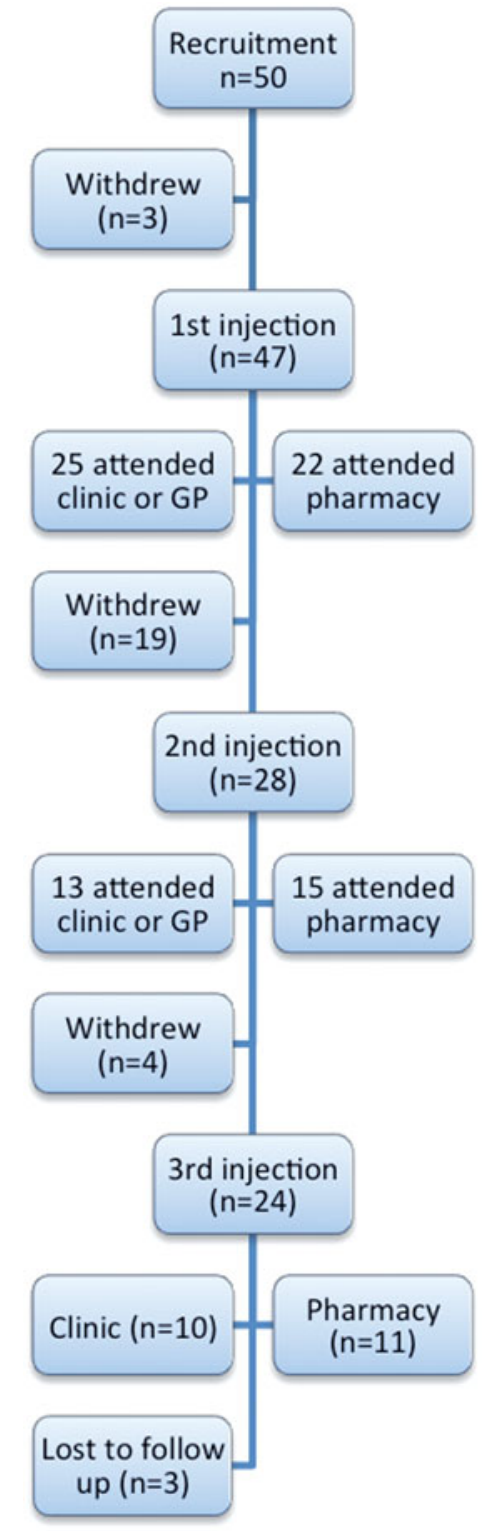

Figure 1. Outcomes and withdrawals.

the pharmacy, two had received one, and four participants stayed in the project but did not manage to receive any injections at the pharmacy. Of the 50 women recruited, 24 received at least one injection at a community pharmacy. Seven received one injection, 10 received two injections and seven received three injections. 22 women completed an exit questionnaire. This was $44 \%$ of participants, $92 \%$ of those who had experienced the intervention. Results are detailed in Tables 3 and 4. Details from the free text comments reflect participants' mixed experiences.

Some participants found the service excellent, and thought it should be available as a routine service. 11 participants had nothing but positive comments.

Very easy and positive experience! Pharmacist great (32-yearold, six months using depo, three injections at pharmacy)

Some women had a more difficult experience, with problems focusing on a few themes. Seven women commented that when they phoned the pharmacy to make an appointment, staff who answered the phone did not know about the service and were unsure how to advise them. This difficulty was reported by women using larger, chain pharmacists, and was more marked at the beginning of the project, but continued throughout the research. Participants found this frustrating and inconvenient, and most women who commented on this did not receive all three injections at the pharmacy.

Three women also expressed concerns about the ability of the pharmacist to give the drug. They commented on the anxiety of the pharmacist, and their apparent lack of familiarity with the injection. This anxiety was mentioned by pharmacists themselves in interviews. One woman said 'I have had the injection many many times before, so the above didn't phase me too much, as I know what to do and what to expect already.' This was a participant in whom the pharmacist attempted the injection four times. She explicitly states that the reason she did not receive three injections at the pharmacy was not because of this.

A high number of participants also commented on the challenge of attending when a trained pharmacist was working. This was more evident in chain pharmacies, where a trained provider might only be available a few days a week.

\section{Working with community pharmacies}

There was a considerable variety in the popularity of pharmacies and the number of injections each pharmacy gave. This was due in part to the movement of pharmacists. Some left the project and were replaced with pharmacists who wanted to continue participating, but others were not replaced. This movement is detailed in Table 5. 
Table 3. advantages of attending community pharmacy.

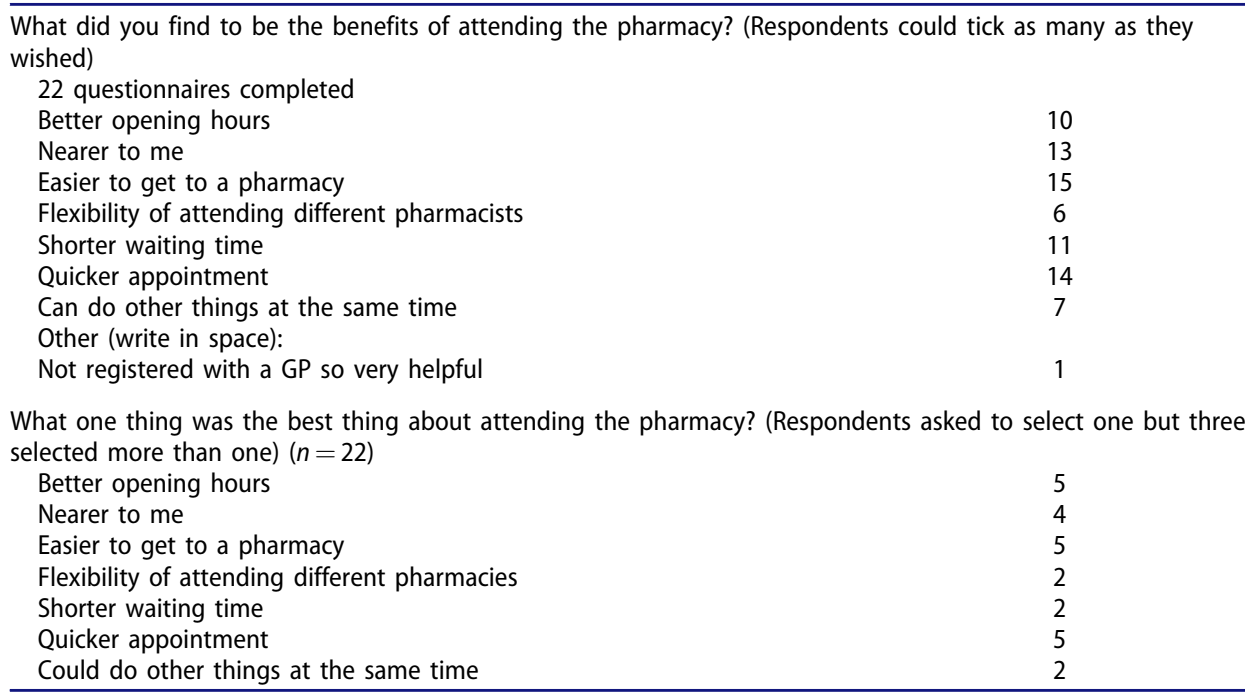

Table 4. Disadvantages of attending the community pharmacy.

What was the worst thing about attending the pharmacy? (Patients could tick as many as they wished)

22 questionnaires completed

Wasn't able to discuss problems I had with the method

Feel more comfortable seeing a nurse or doctor

Wasn't able to discuss other medical problems

Better opening hours at doctors/sexual health clinic

Doctors/sexual health clinic is nearer to me

Easier to get to GP/sexual health clinic

Longer waiting time at the pharmacy

Longer appointment at the pharmacy

Can do other things at the same time in the sexual health clinic

No downside to attending the pharmacy

Other: the pharmacist wasn't there every day

Other: I thought the opening hours would be better

Other: the injection was painful

If you had to select one thing that was the worst thing about attending the pharmacy what would it be? (Respondents asked to select one) $(n=22)$

Wasn't able to discuss problems I had with the method

Feel more comfortable seeing a nurse or doctor

Wasn't able to discuss other medical problems

Better opening hours at doctors/sexual health clinic

Doctors/sexual health clinic is nearer to me

Easier to get to GP/sexual health clinic

Longer waiting time at the pharmacy

Longer appointment at the pharmacy

Can do other things at the same time in the sexual health clinic

No downside to attending the pharmacy

Other: the injection was painful

1
1
1
1
1
1
2
1
1
0
1
1
2
be?
1
2
2
1
1
1
1
0
1
9
1

Table 5. Pharmacy activity.

\begin{tabular}{|c|c|c|c|c|}
\hline & Number of patients seen & Number of injections given & Length of participation & Number of pharmacists trained \\
\hline \multicolumn{5}{|c|}{ Independent pharmacies } \\
\hline Pharmacy 1 & 6 & 9 & Throughout & 2 \\
\hline Pharmacy 2 & 2 & 4 & Throughout & 2 \\
\hline Pharmacy 3 & 0 & 0 & Throughout & 4 \\
\hline Pharmacy 4 & 0 & 0 & $\begin{array}{l}\text { Pharmacist left before any } \\
\text { injections given }\end{array}$ & 1 \\
\hline \multicolumn{5}{|l|}{ Small chains } \\
\hline Pharmacy 5 & 1 & 1 & $\begin{array}{l}\text { Pharmacist left after giving } \\
\text { one injection }\end{array}$ & 1 \\
\hline Pharmacy 6 & 0 & 0 & $\begin{array}{l}\text { One pharmacist left six months } \\
\text { in, another pharmacist was } \\
\text { trained }\end{array}$ & 2 \\
\hline Pharmacy 7 & 0 & 0 & $\begin{array}{l}\text { Joined after } 8 \text { months, left } \\
\text { before project finished }\end{array}$ & 1 \\
\hline \multicolumn{5}{|c|}{ Supermarket chain } \\
\hline Pharmacy 8 & 7 & 13 & Throughout & 1 \\
\hline \multicolumn{5}{|l|}{ Large chain } \\
\hline Pharmacy 10 & 8 & 14 & Throughout & 2 \\
\hline Pharmacy 11 & 1 & 2 & Pharmacist left after 1 year & 1 \\
\hline Pharmacy 12 & 2 & 2 & Throughout & $1^{\mathrm{a}}$ \\
\hline
\end{tabular}

${ }^{a}$ The pharmacist from pharmacy 11 delivered no injections, the trained pharmacist from pharmacy 12 delivered the injections at pharmacy 11. 


\section{Pharmacy interviews}

Interviews were conducted with pharmacists from all but two pharmacies. Pharmacists ranged in experience from just over three years to over 30 years. All pharmacists felt that providing contraceptive services in the pharmacy was a way to improve access for women, as they were conveniently located for people, with better opening hours and quicker appointments than general practitioners or sexual health clinics. They saw themselves as a 'first port of call' and indicated that their contribution to public health care enabled 'freeing up' of doctors. There was a view that providing contraception from the pharmacy would result in GP availability 'to actually deal with emergencies', which would help the health care system, which all pharmacists perceived as being under severe pressure.

Most pharmacists did not perceive the movement of pharmacists or maintaining the service during holiday leave to be a problem. Although they did acknowledge that if they weren't available for a prolonged period: 'you're going to have to find somewhere else so it's not going to work that way', generally, even when questioned specifically, they felt that neither movement of pharmacists or pharmacist absence would interrupt the service delivery and would not prove difficult for participants in the research.

Generally pharmacists were enthusiastic and willing to expand their role. About half of pharmacists interviewed were wholly pleased to develop the ability to administer the contraceptive injection. Some chain pharmacists felt they were not given a choice about role expansion, with pressure from managers to constantly develop new skills so the pharmacy could increase services offered. 'We don't have a choice really.' All chain pharmacists agreed that more managerial support would be necessary to enable long-term delivery of this service.

A factor that the research team had not anticipated was the anxiety which pharmacists felt about delivering the contraceptive injection. Pharmacists stated that this was because administration involved 'breaking the skin'. However, the fact that they were giving contraception also meant the stakes were very high. The importance of contraception to women meant it was crucial to get it right.

Pharmacists felt that the pharmacy was an appropriate place for women to receive contraceptive services and advice. For some pharmacists the fact that the research centred around contraception had been part of the appeal. Through their delivery of emergency hormonal contraception pharmacists were aware of the unmet need for contraception and wanted to help meet it: 'I just think there's so much more we could do.'

\section{Discussion}

\section{Findings and interpretation}

As the high number of withdrawals and some patient comments indicate, this research met a number of challenges. The primary one was the global unavailability of Sayana Press ${ }^{\circledR}$. During this unavailability all patients who had already been recruited were unable to attend the pharmacy for one injection, and some for two injections. Instead they were seen by the research team, and given DMPA-IM. During this time a significant amount of good will was lost with patients. Many that had anticipated attending the community pharmacy but were not able to, withdrew from the project. We cannot say whether the reason that these 17 patients withdrew was definitely the unavailability of Sayana Press ${ }^{\circledR}$. The questionnaire asking participants about reasons for withdrawals was designed and approved by the board of ethics before the study commenced, and the possibility of withdrawing due to unavailability of the contraceptive was not anticipated. The majority of patients who withdrew during this time period cited preferring to attend clinic as the reason for their withdrawal. They declined the option of being notified when Sayana Press ${ }^{\circledR}$ was back in stock so that they could continue to receive injections at the pharmacy once this option was available to them. However it seems likely that this difficulty made the experience less attractive to participants.

In addition, a number discontinued due to side effects. This is a common reason to discontinue all contraception, including the contraceptive injection [7]. In addition, in September 2015 Sayana Press ${ }^{\circledR}$ was licensed in the UK for self-injection [14]. The centre from where recruitment took place began offering this service in July of 2016. Three participants withdrew to learn self-injection rather than continue attending the pharmacy.

Working with community pharmacies presented other challenges. Some pharmacists working for commercial chains took months to be able to deliver the contraceptive injection. This was not the case for any independent pharmacists. Pharmacies did not have any protocols or procedures for practice in the context of research, and all pharmacists except one were new to clinical research. Each commercial chain had forms that needed to be signed by differing levels of management to enable pharmacists to use the patient group direction. In addition pharmacists involved had a number of pressures that inevitably impacted the resources available for them to spend on this research, particularly as they were not given any extra time or financial compensation. The time taken for each pharmacist to receive full approval from each level of management delayed progress and consequently a number of participants that anticipated receiving their injection at a chain pharmacy needed to attend a clinic instead. This break in anticipated service delivery may be another explanation for the high withdrawal rate seen. This frustration was reflected in the participant comments, and may be a further explanation for the high withdrawal rate seen.

A further unexpected challenge was the number of times that trained pharmacists were moved to different pharmacies. The participating pharmacist might only be working at the relevant pharmacy one or two days a week. As a consequence, a number of patients ended up contacting the research team to ask for a clinic appointment, as they were anxious about not receiving their injection in time, and putting themselves at risk of unplanned pregnancy.

The number of people working in each pharmacy meant that the full staff was not always aware that the service was being offered, or potentially offered. Participants attempting to make their appointment were informed on more than one occasion that this service was not offered. This was discouraging and generated some discontent, as is demonstrated in the participant comments. Attempts to rectify this were made by repeated site visits by 
investigators, but a high degree of staff turnover meant that many staff remained unaware of their pharmacy's participation. When asked, pharmacists involved felt confident that all staff in the pharmacy were aware of the service, and declined further visits or meetings, but this picture did not match patient's comments. Dissemination of information is a challenge in large pharmacy chains and is an important aspect of the feasibility of the research being expanded. It is likely that the uncertainty was a consequence of this being a small research project rather than a service offered generally, but demonstrates the difficulty of bringing about service change, particularly within large pharmacies employing many pharmacists.

\section{Strengths and weaknesses of the study}

A strength of this study is that very limited research has been carried out into patients receiving contraception injections at the community pharmacy. Those that do have concentrated on using a single pharmacy, or involved many but found that by far the majority of injections were given at a very small number of pharmacies $[4,5,15]$. Our results replicate this finding, as the majority of participants also attended three pharmacies in the study.

The weaknesses of the study are the small sample size and the high number of withdrawals. Withdrawals were for a number of reasons but the global unavailability of the injection being used was almost certainly a contributory factor. The training and the way the research was set-up did not adequately cover the amount of pharmacist movement within the study. These aspects could be minimised in further studies.

\section{Differences in results and conclusions in relation to other studies}

Little has been published on this subject. Picardo et al. conducted the only randomised controlled trial, where 50 women were randomised to receive contraceptive injections either at their clinic or at a nearby pharmacy. Women were less likely to return to the pharmacy than the clinic for both their second and third injection, although there was no reported difference in satisfaction with location or convenience. Our findings were in keeping with this, with more injections being given in the clinic than at a pharmacy [4]. Monastersky et al. partnered 27 pharmacists with 19 clinics, offering 69 women 143 contraceptive injections. There is no information on discontinuation or withdrawal rates so it is difficult to compare our results, but clinicians involved concluded, as we did, that the programme would benefit from all local pharmacies carrying the service [5]. Ferreira et al examined women who had received three-monthly injections at the pharmacy compared to women who had received sixmonthly injections at the pharmacy, with alternate threemonth injections being given at a location closer to home, including pharmacies. Women who received this service were more likely to continue with the method, and less likely to become pregnant [15]. Some of the difficulties we experienced in working with pharmacists, notably their lack of experience in clinical research, have also been documented in the literature [16].

\section{Relevance of the findings: implications for clinicians and policy makers}

In the future, the licensing of Sayana Press ${ }^{\circledR}$ for self-administered injection [14] is likely to impact significantly upon the utility of exploring pharmacy administration. The main concern that women cite when discussing receiving health care in the community pharmacy setting is lack of privacy, with resulting anxiety about confidentiality [17-19]. The main advantages are cited as convenience and speed and ease of access [20,21]. Giving patients the ability to selfadminister in the privacy of their own homes at a time that suits them would confer more advantages in these areas than attending a pharmacy does, as evidence on self-injection suggests [22-24].

It may be that there are small numbers of women who do not wish to self-inject, and the pharmacy could offer this resource. The response of participants and pharmacists, particularly in chain pharmacies, suggests that any contraceptive service offered would have a greater chance of success if the service was offered by the majority of pharmacists in all pharmacies. The confusion of some staff and the sporadic nature of the injections seems to have had a negative impact on the experience of patients. In particular the limited pharmacist availability hindered access, and increased access to this method was the rationale for initiating the research. If offering the contraceptive injection at the pharmacy became routine then it is possible awareness would spread and both pharmacists and pharmacy staff would become more comfortable with the concept. The influenza vaccination is a good example of an injection delivered successfully by pharmacies $[25,26]$. A key difference seems to be that this was instigated by high-level management within pharmacies, and a number of systems were put in place to ensure that vaccinations could be delivered to customers.

\section{Unanswered questions and future research}

It is difficult to know to what extent the problems encountered are a result of specific systems in place. It is possible that repeating the project but addressing some of the training needs identified by pharmacists and staff working in pharmacy could generate a more successful response. The comments about 'shaky hands' and multiple attempts being needed to give injections suggests that the training was not adequate for pharmacist confidence, even if it was deemed adequate for competence. A rolling programme of training for pharmacists to attend prior to giving an injection might alleviate some of these anxieties. Ideally future research would provide adequate funding to enable pharmacists to attend further training in their paid working hours, for example by providing relief pharmacist cover. An alternative option would be for a research student or nurse to attend each pharmacist prior to each injection to repeat the procedure and answer any questions. Our approach was to provide an open line of communication that the pharmacists could use if they wanted further training. This option was only taken up by one pharmacist who had additional supervision and training at their pharmacy. However, if the research were to be repeated, routinely offering additional training, at least in the early stages, might increase pharmacist confidence. It should however be noted here 
that, although some participants commented on pharmacist anxiety, none offered this as a reason for withdrawal from the research.

When we invited the pharmacists to participate, only one pharmacist from each pharmacy wished to develop the skill, but this might be different if more financial support had been available or more in-depth training. Training every pharmacist working at each pharmacy involved in service delivery could be a useful approach in further studies, as this would negate the problem of advertised opening hours being so different to trained pharmacist availability. However, the largest chain pharmacies had a very large number of pharmacists who could work in each pharmacy, and therefore this would be very challenging unless delivering the contraceptive injection became a core pharmacist skill, as influenza vaccination has now done $[26,27]$. Such a change in pharmacy services would need managerial input, as well as the support of local public health authorities, and this might be difficult to achieve without compelling research to demonstrate its efficacy and acceptability. Nevertheless, enthusiastic support at a managerial level would be considerably more likely to lead to new service implementation [28].

Ultimately a randomised controlled trial, where patients are randomised to receive injections either from the pharmacy or from a clinic or GP, would be the most robust way to assess injections received, continuity rates and patient experiences.

\section{Conclusions}

Delivery of the subcutaneous contraceptive injectable from a community pharmacy may be feasible but availability of sufficient numbers of pharmacists trained in this technique is necessary for a robust model of service delivery.

\section{Funding}

This study was funded by an independent investigator-initiated research grant from Pfizer Ltd., makers of Sayana Press ${ }^{\circledR}$.

The PhD of the first author was funded by an unrestricted grant from HRA Pharma.

\section{ORCID}

R. Heller (D) http://orcid.org/0000-0003-3584-0141

\section{References}

[1] Todd A, Copeland A, Husband A, et al. Access all areas? An area-level analysis of accessibility to general practice and community pharmacy services in England by urbanity and social deprivation. BMJ Open. 2015;5:e007328.

[2] Department of Health. Choosing health: making healthier choices easier. London: Department of Health; 2004.

[3] Department of Health. Choosing health through pharmacy. London: Department of Health; 2005.

[4] Picardo C, Ferreri S. Pharmacist-administered subcutaneous depot medroxyprogesterone acetate: a pilot randomized controlled trial. Contraception. 2010;82:160-167.

[5] Monastersky MNJ, Landau SC. Pharmacy and clinic partnerships to expand access to injectable contraception. J Am Pharm Assoc (2003). 2006;47:527-531.
[6] Trussell J. Contraceptive failure in the United States. Contraception. 2011;83:397-404.

[7] Moreau C, Cleland K, Trussell J. Contraceptive discontinuation attributed to method dissatisfaction in the United States. Contraception. 2007;76:267-272.

[8] Kaunitz AM, Peipert JF, Grimes DA. Injectable contraception: issues and opportunities. Contraception. 2014;89:331-334.

[9] Potter LS, Dalberth BT, Cañamar R, et al. Depot medroxyprogesterone acetate pioneers: a retrospective study at a North Carolina health department. Contraception. 1997;56:305-312.

[10] Paul CSDCG, Williams S. Depot medroxyprogesterone acetate. Patterns of use and reasons for discontinuation. Contraception. 1997;56:209-214.

[11] Faculty of Sexual and Reproductive Healthcare. UK Medical Eligibility Criteria for Contraceptive Use (UK MEC 2016). London: Faculty of Sexual and Reproductive Healthcare; 2016.

[12] Heller R, Cameron ST. Evaluating the attractiveness of the availability of injectable progestogen contraceptives at the community pharmacy setting in the United Kingdom. Int J Pharm Pract. 2016;24:247-252.

[13] The Scottish Government. Scottish Index of Multiple Deprivation 2012: a national statistics publication for Scotland. Edinburgh: The Scottish Government; 2016.

[14] Faculty of Sexual and Reproductive Healthcare. CEU statement on self-administration of Sayana Press. London: Faculty of Sexual and Reproductive Healthcare; 2015.

[15] Ferreira JM, Bottura BF, Gonçalves MP, et al. Comparison of two strategies for the administration of injectable depot medroxyprogesterone acetate: among women who returned to a family planning clinic at three- or six-month intervals. Eur J Contracept Reprod Health Care. 2016;21:408-411.

[16] Black K, Anderson C, Kubba A, et al. Involving pharmacists in sexual health research: experience: experience from an emergency contraception study. J Fam Plann Reprod Health Care. 2009;35:41-43.

[17] Saramunee K, Krska J, Mackridge A, et al. How to enhance public health service utilization in community pharmacy?: General public and health providers' perspectives. Res Social Admin Pharm. 2014;10:272-284.

[18] Lambeth Lewisham and Southwark Health Action Zone. A Timely Service: A Lambeth, Southwark \& Lewisham Health Action Zone project on access to emergency hormonal contraception via accredited community pharmacies. London: Lambeth Lewisham and Southwark Health Action Zone; 2002.

[19] Anderson C, Blenkinsopp A, Armstrong M. Feedback from community pharmacy users on the contribution of community pharmacy to improving the public's health: a systematic review of the peer reviewed and non-peer reviewed literature 1990-2002. Health Expect. 2004;7:191-202.

[20] Barlassina L. Views and attitudes of oral contraceptive users towards their availability without a prescription in the Republic of Ireland. Pharm Pract (Granada). 2015;13:565.

[21] Greene D, Sharon F, Landau C, et al. Pharmacy access to emergency contraception in California. Perspect Sex Reprod Health. 2006;38:46-52.

[22] Kim CR, Fønhus MS, Ganatra B. Self-administration of injectable contraceptives: a systematic review. BJOG. 2016;124:200-208.

[23] Beasley A, O'Connell White K, et al. Randomized clinical trial of self versus clinical administration of subcutaneous depot medroxyprogesterone acetate. Contraception. 2014;89:352-356.

[24] Cameron ST, Glasier A, Johnstone A. Pilot study of home selfadministration of subcutaneous depo-medroxyprogesterone acetate for contraception. Contraception. 2012;85:458-464.

[25] Orrell C, Leong A, Boyd M, et al. An evaluation of pharmacy customer views of services that should be available from community pharmacy. Roy Pharm Soc Ann Conf. 2015;23:59-60.

[26] Anderson C, Thornley T. It's easier in pharmacy': why some patients prefer to pay for flu jabs rather than use the National Health Service. BMC Health Serv Res. 2014;14:35.

[27] Brown D, Portlock J, Rutter P. Review of services provided by pharmacies that promote healthy living. Int J Clin Pharm. 2012;34:399-409.

[28] Grol R, Wensing M. What drives change? Barriers to and incentives for achieving evidence-based practice. Med J Aust. 2004;180:S57-S60. 


\title{
Routine provision of intrauterine contraception at elective cesarean section in a national public health service: a service evaluation
}

\author{
REBECCA HELLER ${ }^{1,2}$ (D) ANNE JOHNSTONE ${ }^{2}$ \& SHARON T. CAMERON ${ }^{1,2}$ \\ ${ }^{1}$ NHS Lothian, Chalmers Center, Edinburgh, and ${ }^{2}$ University of Edinburgh, Edinburgh, UK
}

Key words

Contraception, cesarean, contraceptive devices, postpartum care, intrauterine contraception

\section{Correspondence}

Rebecca Heller, ST1 in Community Sexual and Reproductive Health, NHS Lothian, University of Edinburgh, Chalmers Center, 2A Chalmers Street, Edinburgh, EH3 9ES Edinburgh, UK.

E-mail: rebecca.heller@ed.ac.uk

\section{Conflict of interest}

R.H.'s fellowship salary was paid by an unrestricted grant from HRA Pharma. The other authors have stated explicitly that they have no conflicts of interest in connection with this article.

Please cite this article as: Heller $\mathrm{R}$, Johnstone A, Cameron ST. Routine provision of intrauterine contraception at elective cesarean section in a national public health service: a service evaluation. Acta Obstet Gynecol Scand 2017; 96:1144-1151.

Received: 2 March 2017 Accepted: 29 May 2017

DOI: 10.1111/aogs.13178

\begin{abstract}
Introduction. We conducted a prospective health service evaluation to assess the feasibility and acceptability of routinely offering insertion of intrauterine contraception at cesarean section in a maternity setting in the UK. Material and methods. One month before scheduled cesarean section, women were sent information about postpartum contraception including the option of insertion of an intrauterine contraception at cesarean. Women choosing intrauterine contraception (copper intrauterine device or levonorgestrel intrauterine system) were followed up in person at six weeks, and telephone contact was made at three, six and 12 months postpartum. Our main outcome measures were uptake of intrauterine contraception and complications by six weeks. Secondary outcomes were continuation and satisfaction with intrauterine contraception at 12 months. Results. 120/877 women opted to have intrauterine contraception (13.7\%), of which 114 were fitted. By six weeks, there were seven expulsions $(6.1 \%)$. The expulsion rate by one year was $8.8 \%$. There were no cases of uterine perforations and one case of infection $(0.8 \%)$. Follow-up rates were $82.5 \%$ at 12 months, and continuation rates with intrauterine contraception at 12 months were $84.8 \%$ of those contacted. At 12 months, $92.7 \%$ of respondents asked were either 'very' or 'fairly' happy with their intrauterine contraception. Conclusions. Routine provision of intrauterine contraception at elective cesarean for women in a public maternity service is feasible and acceptable to women. It is associated with good uptake and good continuation rates for the first year. This could be an important strategy to increase use of intrauterine contraception and prevent short interpregnancy intervals and unintended pregnancies.
\end{abstract}

Abbreviations: IUC, intrauterine contraception; LARC, long-acting reversible contraception; LNG-IUS, levonorgestrel intrauterine system; NHS, National Health Service.

\section{Introduction}

There is growing recognition of the need to improve uptake of contraception in the immediate postpartum period. Up to $50 \%$ of women have resumed sexual activity by six weeks postpartum and ovulation may occur as early as three weeks postpartum in non breast-feeding women (1). A UK study reported that one in 13 women requesting termination had conceived the pregnancy within one year of childbirth (2), and one in 13 women who gave birth had conceived within one year of childbirth. An interpregnancy interval of less than 12 months increases the risk of complications, including preterm birth, stillbirth and neonatal death (3).

Long-acting reversible contraception (LARC) methods such as the copper intrauterine device (Cu-IUD) and levonorgestrel intrauterine system (LNG-IUS), and the 
subdermal implant are known to be the most effective methods of preventing unintended pregnancy (4). The prevalence of use of intrauterine contraception (IUC) in the UK is approximately $8.7 \%$ (5). Traditionally, women choosing IUC postpartum may require several visits with a healthcare provider before insertion (6). Each visit may represent a significant barrier for women who are likely to be recovering from childbirth (particularly after a cesarean section) and caring for a new baby. There is good evidence that insertion of IUC at cesarean is safe, convenient and acceptable, and has high continuation rates (7-18). However, this information comes mainly from low-income countries and the USA. This practice has not been widely reported in Europe, and there are no data from the UK. With a cesarean section rate of $30 \%$ in Scotland (19) and 27\% in England (20) in 2015/2016, there is the potential to offer IUC at cesarean to a significant proportion of women. In the UK, maternity care and sexual healthcare, including all contraception, is free of charge, delivered through the National Health Service (NHS).

NHS Lothian (Edinburgh and the surrounding region, Scotland) began a series of pilot initiatives in 2013 to improve the uptake of postpartum contraception. This involved the introduction of antenatal contraceptive counseling by midwives and provision of contraception to women living in one area of the region from the maternity service after delivery (21). This current study aimed to investigate the feasibility of routinely offering all women within this region who were undergoing an elective cesarean section the opportunity to have IUC insertion at this time, and to assess uptake, complications, continuation rates and acceptability.

\section{Material and methods}

A training package and video were developed that included the technique of insertion of IUC at cesarean section, medical eligibility criteria for contraceptive use (22), contraindications to IUC postpartum, and what post-procedure counseling with women should include. The team also produced checklists that obstetricians could use for counseling women about IUC, detailing the risks, benefits and possible side effects, and written information to give to women about what to expect after IUC insertion and what follow up was necessary. This material was presented to all consultants and obstetric trainees working at the two maternity hospitals in the region (approximately 35 doctors). Consultants underwent one individual training session and registrars observed the video as part of their ongoing training. Ongoing peer supervision in theater was encouraged and adopted until all obstetricians were trained; this took approximately four months.
Obstetricians were instructed that following delivery of the placenta, the IUC should be removed from its inserter and placed at the fundus through the uterine incision. They were instructed to use artery forceps to guide the threads of the device towards the cervix, and through the cervix if possible. Threads of the device were not cut. The uterus, sheath and skin were then sutured as per the obstetrician's normal practice. In addition, training on contraception and information about IUC insertion at cesarean was also provided to hospital midwives.

From the 22 June 2015, information about all types of postpartum contraception was included with the routine information on an elective cesarean section that was sent to women three to four weeks before their scheduled cesarean date. This included a letter stating that if women wished to use IUC for contraception postnatally, they might be able to choose to have it inserted during their cesarean section. Women were invited to discuss this option with their obstetrician in advance of delivery. This was in addition to the routine antenatal contraceptive counseling that some women in the region received at that time from a midwife at 22 weeks (21). The telephone numbers of the evaluation team were included so that women could contact the evaluation team if they had any unanswered questions about IUC at cesarean. When women gave consent for their delivery on the day of cesarean section, those who had made the prior decision to have IUC also gave written consent for insertion of IUC.

Following delivery all women who had elective cesareans were reviewed by obstetricians. Both obstetric and midwifery staff provided verbal and written information about the device that was inserted and what to expect in coming weeks. All women who had IUC fitted were identified by the evaluation team using electronic maternity records (TRAK). These women were contacted and offered a six-week IUC thread check at their local hospital or sexual health center with the evaluation team (A.J., a research nurse, and R.H., a research fellow who is an obstetric and gynecology trainee). At this visit, women had the opportunity to answer any further questions

\section{Key Message}

Short interpregnancy intervals are associated with poorer obstetric outcomes, so providing effective contraception immediately postpartum is an essential part of healthcare. Routinely offering women undergoing cesarean section, intrauterine contraception provides a safe solution with high satisfaction and continuity rates one year later. 
about IUC. They underwent a speculum (RH) to look for the threads of the IUC and trim them if necessary. If no threads were seen, an ultrasound scan was conducted to ensure the device was in situ. A partial expulsion was diagnosed if the stem of the device was seen on clinical speculum examination, or if an ultrasound scan revealed the device to be in the cervical canal. (A complete expulsion was diagnosed if the woman reported the device as expelled, or if no threads were seen, no device was visible on ultrasound, and no device was visible on abdominal $\mathrm{x}$-ray.) Women were also asked how satisfied they were with having the IUC inserted at cesarean.

Women were then contacted by phone or text at three, six and 12 months to determine continuation rates and satisfaction with the IUC as a method of contraception. Our main outcome measures were uptake of IUC and complications by six weeks. Secondary outcomes were continuation and satisfaction with IUC at 12 months.

In addition to the information gathered at follow up, the regional hospital database was reviewed for each woman to ensure that data on any complications was accurate. For all women who were lost to follow up, both the regional hospital database and the local sexual health database were examined to obtain any further information about the presence or removal of IUC, as well as any further pregnancies. Sample size was not determined based on an a priori hypothesis; we continue to collect data on women that undergo this procedure. We report here data from those women who have completed 12 months follow up.

\section{Ethical approval}

The local Quality Improvement Teams for women's services and sexual and reproductive health approved the project. The scientific officer of the local ethics committee reviewed the project proposal and confirmed that ethical approval was not required as this was a health service evaluation project.

\section{Results}

Between 13 July 2015 and 2 March 2016, 877 women were scheduled for cesarean section and were sent information about having IUC inserted at delivery. In all, 120 women chose this option (13.7\%). Demographics of women who chose IUC are detailed in Table 1.

A total of 114 devices were inserted at the time of cesarean, 100 LNG-IUS and $14 \mathrm{Cu}-\mathrm{IUD}$. Two women changed their minds. Four IUC could not be inserted due to postpartum hemorrhage; of these, the women were known to have attended the local sexual health service for subsequent insertion of IUC $(n=3)$ or insertion of a
Table 1. Demographics.

\begin{tabular}{lc}
\hline Age, median (range) & $33(21-41)$ years \\
Body mass index at booking, \\
median (range) \\
$\begin{array}{c}\text { Parity } \\
0\end{array}$ \\
1 & $9(7.5 \%)$ \\
2 & $80(66.6 \%)$ \\
3 & $26(21.6 \%)$ \\
4 & $2(1.7 \%)$ \\
$4+$ & $2(1.7 \%)$ \\
Scottish Index of Maternal Deprivation (SIMD) $(23)$ \\
SMID 5 (affluent) \\
4 & $33(27.5 \%)$ \\
3 & $22(18.3 \%)$ \\
2 & $20(16.6 \%)$ \\
1 (deprived) & $24(20.0 \%)$ \\
\hline
\end{tabular}

subdermal contraceptive implant $(n=1)$ (Figure 2). In all, 103 women attended the six-week follow up, and a further nine were contacted at this time by phone. The percentage of women with information available at each interval is detailed in Figure 1.

Outcomes of IUC insertion are detailed in Figure 2. Of the 114 women who had IUC inserted at cesarean (88.5\%), 100 attended for a follow up at six weeks. A further three women who had not had a device inserted came for insertion at six weeks. Fourteen women attended earlier than six weeks due to noticing long threads or threads external to the vagina. One of these women was found to have a partial expulsion and the device was removed and a new IUC replaced. All others had the threads trimmed to $2 \mathrm{~cm}$ from the os.

There were 10 expulsions, seven by six weeks following insertion, one at seven weeks, one at 14 weeks, and one at 28 weeks. One of the early expulsions was unnoticed but was presumed to be before six weeks, as no device was seen at the six-week scan (details below). The 10 expulsions included five partial expulsions. Four Cu-IUDs were expelled and six LNG-IUSs. All complete expulsions were noticed by women except in one case. Of the 10 expulsions, six women were fitted with a replacement IUC. In four cases, IUCs were removed accidentally by women. Two happened before women were seen for their six-week thread check, when their threads had not been trimmed. Two others were pulled out with tampons. One woman had her device replaced.

There was one case of endometritis diagnosed at the six-week check (pain plus Escherichia coli infection confirmed on high vaginal swab); this was treated with oral antibiotics and the device was left in situ. Another device was removed at two days post-insertion due to an initial suspicion of endometritis, but microbiological investigation was negative, and the woman was subsequently 


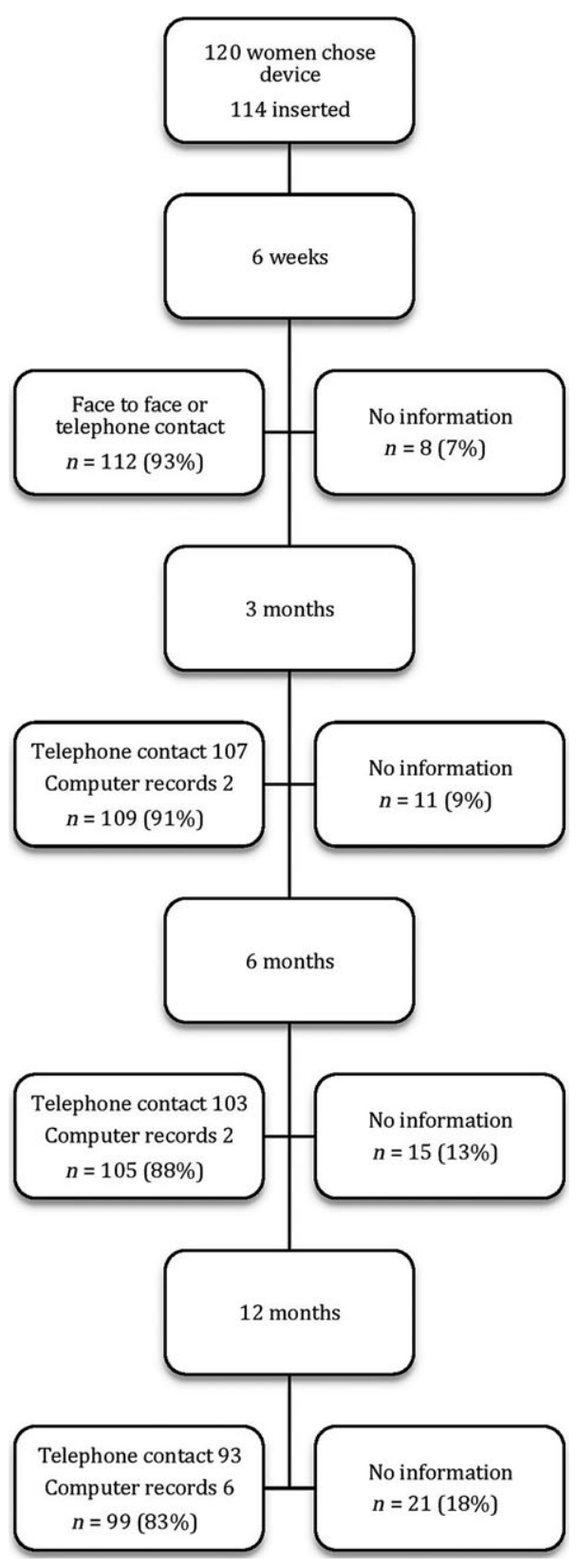

Figure 1. Percentage of women with information available at follow up.

diagnosed with pyelonephritis (a further device was inserted eight months later).

Complications that had occurred by six weeks included seven expulsions (three partial and four complete), two accidental removals and one case of endometritis. There were three removals by six weeks, one for suspected infection and two at patient request.

Of 97 women who attended believing they had an IUC in situ, threads were visible in 49 cases (50\%). Ultrasound confirmed intrauterine placement in all but two women with missing threads. One woman was noted to have an intracervical device, this was removed and alternative contraception started; this is counted in our data as a partial expulsion. Another woman had no device as seen on ultrasound. Unfortunately, despite multiple efforts she did not attend for abdominal x-ray. She did attend clinic six months after insertion with an unplanned pregnancy for a termination, and was considered to have a unrecognized complete expulsion. Six women did not attend the six-week thread check but were followed up by telephone and either reported that they could feel threads or had attended their general practitioner for a confirmation that the IUC threads were visible. Of the four women who could not have an IUC inserted at cesarean, three of them attended for IUC insertion at six weeks.

Continuation rates are detailed in Table 2. Percentages are detailed both from the total number of devices planned, using intention-to-treat and assuming that everyone lost to follow up is no longer using IUC for contraception, and as a percentage of number of women contacted. At 12 months, 93 women were contactable, and information about a further six was gained from the clinical databases, a follow up rate of $82.5 \%$. As a proportion of all 117 women who had the device fitted either at cesarean $(n=114)$ or afterwards $(n=3), 71.8 \%$ were known to have a device in situ at 12 months $(n=84)$.

Of 117 devices inserted, 15 have been removed (12.8\%); 14 removals were of devices inserted at cesarean and one of a device inserted 10 weeks postpartum. Removals have been from two days after insertion to 27 weeks, all were LNG-IUS devices. Reasons for removal included disliking bleeding patterns $(n=5)$, headache $(n=1)$, disliking a foreign body in the uterus $(n=1)$, recurrent thrush $(n=1)$, pain during intercourse $(n=1)$, postpartum hemorrhage $(n=1)$, not liking the method $(n=1)$, believing the method affected milk production $(n=1)$ and wanting to conceive again $(n=1,10$ months in situ). One removal was due to placement concerns. An ultrasonographer reported the IUC to be embedded in the myometrium, but it was removed easily and replaced. One woman whose device was removed due to an infection concern subsequently had the IUC replaced. All removals were straightforward outpatient procedures, including three cases where threads were not visible. Of note, one woman who chose to have her LNG-IUS removed and commenced a less effective method (the combined hormonal contraceptive patch) had an unintended pregnancy two months later.

After one year, 82 women still using the device were asked about their satisfaction with the device; $92.7 \%$ of them were 'very' or 'fairly' happy with the IUC $(n=76)$. Further satisfaction rates are detailed in Table 3. 


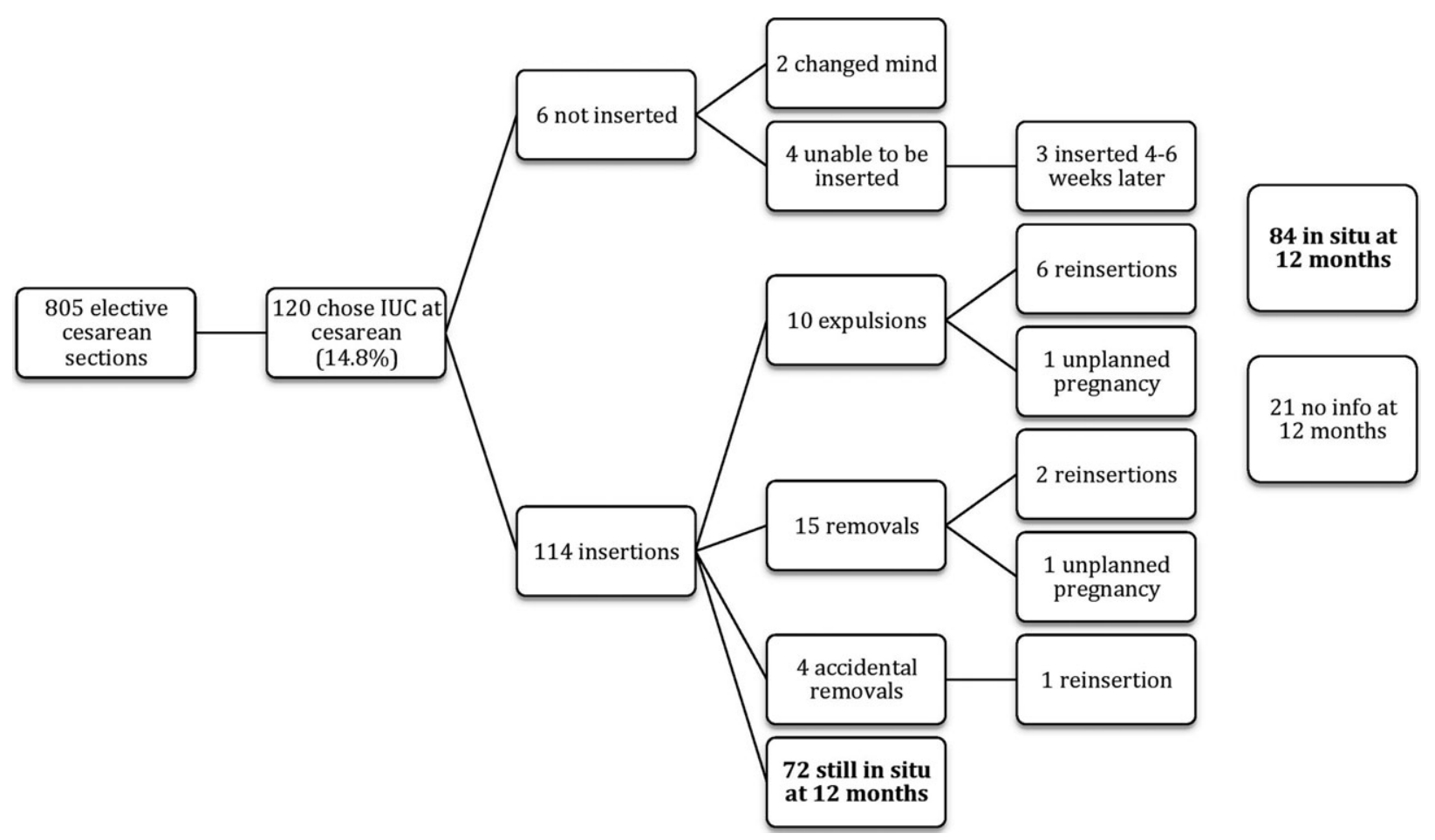

Figure 2. Outcomes of participants.

Table 2. Continued use of intrauterine contraception (IUC).

\begin{tabular}{|c|c|c|c|c|c|}
\hline$n=120$ & At cesarean section & 6 weeks & 3 months & 6 months & 12 months \\
\hline Number of women contacted/information available & 120 & 112 & 109 & 105 & 99 \\
\hline Using IUC & 114 & $111^{\mathrm{a}}$ & 96 & 91 & $84^{\mathrm{b}}$ \\
\hline Continuation rates as percentage of those with known status & $95 \%$ & $99 \%$ & $88 \%$ & $87 \%$ & $85 \%$ \\
\hline Continuation rates as percentage of 120 planned insertions & $95 \%$ & $93 \%$ & $80 \%$ & $76 \%$ & $70 \%$ \\
\hline
\end{tabular}

a 114 devices inserted at cesarean, 3 removals, 7 expulsions, 4 replacements, 3 new insertions.

b72 still in situ, 6 expulsions replaced, 2 removals replaced, 1 accidental removal replaced, 3 new insertions.

\section{Discussion}

Routinely offering insertion of IUC at the time of elective cesarean section was popular among women, with more than one in eight women in this cohort choosing this option, the majority opting for an LNG-IUS. In addition, the study confirmed the low complication rate associated with insertion of IUC at this time and an expulsion rate in keeping with that of insertion of IUC in women who are not postpartum (24). Furthermore, satisfaction rates among women with IUC insertion at cesarean were high, and satisfaction and continuation with the method remained high at 12 months post-insertion.

To our knowledge this is the first paper from Western Europe to evaluate insertion of IUC at the time of elective cesarean section, routinely offered in a public health setting. Our loss to follow up was low, and reviewing regional databases minimized the risk of missing any serious complications associated with IUC insertion. In addition, there are few existing data in the literature regarding women's acceptability of IUC insertion at this time. It is a significant strength of our paper that we examined routine insertion of IUC in a setting where all contraception is free. A number of other studies have made IUC free for the purposes of research, in a setting where this would normally entail an extra cost for patients. However, in this NHS population, cost is not an incentive to chose a contraceptive method, so removing the barrier of attending a clinic at a later date in the postpartum period may have a proportionately greater impact on women's choice of IUC at cesarean.

This was an evaluation of a new service and so the findings are likely to represent uptake of IUC in similar public maternity settings. Our study is limited, however, 
Table 3. Satisfaction rates.

\begin{tabular}{|c|c|c|c|c|}
\hline \\
\hline \multicolumn{5}{|c|}{$\begin{array}{l}\text { Satisfaction with insertion taking place at the time of cesarean section }(n=114) \text { (asked at six-week follow up) } \\
\text { Number of women asked }\end{array}$} \\
\hline Very happy & & & & $74(76 \%)$ \\
\hline Fairly happy & & & & $21(21 \%)$ \\
\hline Neither happy nor unhappy & & & & $3(3 \%)$ \\
\hline Fairly unhappy & & & & 0 \\
\hline \multirow[t]{2}{*}{ Very unhappy } & & & & 0 \\
\hline & $\begin{array}{l}\text { 6-week thread } \\
\text { check }\end{array}$ & $\begin{array}{l}\text { 3-month } \\
\text { follow up }\end{array}$ & $\begin{array}{l}\text { 6-month } \\
\text { follow up }\end{array}$ & $\begin{array}{l}\text { 12-month } \\
\text { follow up }\end{array}$ \\
\hline \multicolumn{5}{|l|}{ Satisfaction with intrauterine contraception device } \\
\hline Number of women with intrauterine contraception in situ & $111^{\mathrm{a}}$ & 96 & 91 & 84 \\
\hline Number of women asked & 99 & 92 & 89 & 82 \\
\hline Very happy & $42(42 \%)$ & $67(72 \%)$ & $61(69 \%)$ & $64(78 \%)$ \\
\hline Fairly happy & $43(43 \%)$ & $21(23 \%)$ & $22(25 \%)$ & $12(15 \%)$ \\
\hline Neither happy nor unhappy & $14(14 \%)$ & $4(4 \%)$ & $4(4 \%)$ & $5(6 \%)$ \\
\hline Fairly unhappy & 0 & 0 & 0 & $1(1 \%)$ \\
\hline Very unhappy & 0 & 0 & $2(2 \%)$ & $1(1 \%)$ \\
\hline
\end{tabular}

Percentages are given as a percentage of women asked.

ancluding those who had had a device expelled or removed, and reinserted.

by the fact that this service evaluation relates to a single region. The percentage of women choosing IUC and the satisfaction rates may differ in other populations. We have limited data on removals, as only a small number of women have had their device removed. It is reassuring that removal was straightforward in those women who requested it.

As has been argued elsewhere, the main advantage of insertion of IUC at cesarean section is the benefit it confers in terms of uptake and continuity (25). In addition, the procedure is convenient for both women and health services, and avoids a potentially more difficult insertion through a possibly narrow os weeks later. This is particularly relevant given the evidence that the risk of uterine perforation is increased in the first 36 weeks postpartum, with breastfeeding at time of insertion associated with a sixfold increased risk (26). The number of women choosing IUC was considerably higher than was previously found in a similar maternity population, when insertion at cesarean section was not routinely available (2).

Expulsion rates in our study were low, and when expulsion did occur, it was recognized in all but one case, and most these women chose to have another device inserted. A potential challenge with IUC insertion at cesarean is that a significant proportion of women will not have visible threads at six weeks. In our study this was half of all cases. This indicates that an important component of making insertion of IUC at cesarean routine practice is a system ensuring rapid access to ultrasound scans to confirm that the IUC is in situ. This could be either at a hospital or clinic or by direct referral to sexual health centers that have ultrasound capabilities.
A small number of women in our study presented with very long threads, but trimming the threads can be easily managed by any healthcare practitioner who is competent to conduct a cervical smear.

Insertion of IUC at cesarean section is a relatively straightforward procedure. Obstetricians are skilled operators and insertion of the IUC at cesarean is not complicated. Indeed, it is more straightforward than female sterilization at the time of cesarean, and could in fact be offered to women as an alternative to this practice, particularly as sterilization is a permanent procedure. Training of obstetricians was easy and required a small number of dedicated trainers only (the evaluation team). Training for midwives to ensure a basic knowledge about IUC and dispel any myths was also required, although some of this could be delivered online or in video format rather than in face-to-face teaching sessions (21).

Robust processes need to be in place for appropriate follow up post IUC insertion to succeed; thus a close working partnership between maternity, sexual health services and general practitioners is important to make routine insertion of IUC at cesarean achievable and successful.

\section{Conclusion}

Routine provision of IUC at cesarean section is feasible with a simple training package for maternity staff. It is both safe, with low complication rates, and highly acceptable to women, with good uptake and good continuation rates. Any increase in LARC in the postpartum setting has the potential to prevent unintended pregnancy and 
enable women to achieve healthy inter-pregnancy intervals. We would propose that this option is routinely offered to women as part of good maternity care (27).

\section{Acknowledgments}

We are grateful to Allyn Dick for assistance with compiling of maternity figures, to Stephen Punton of Medical Photography for preparation of video materials, and to the obstetricians, midwives and patients involved in this study. We are especially grateful to Dr. Shiona Coutts for assistance with data capture and Dr. Sarah Cooper and the patient concerned for permitting filming of the teaching video.

\section{Funding}

Some funds for the evaluation came from NHS Lothian Sexual Health Strategy Funding. R.H.'s fellowship salary was paid by an unrestricted grant from HRA Pharma. Funding was also provided by Wellbeing of Women, and Chief Scientist Office, Scottish Government.

\section{References}

1. Jackson E, Glasier A. Return of ovulation and menses in postpartum nonlactating women: a systematic review. Obstet Gynaecol. 2011;117:657-62.

2. Heller R, Cameron S, Briggs R, Forson N, Glasier A. Postpartum contraception: a missed opportunity to prevent unintended pregnancy and short interpregnancy intervals. J Fam Plann Reprod Health Care. 2016;42:93-8.

3. Smith GCS, Pell JP, Dobbie R. Interpregnancy interval and risk of preterm birth and neonatal death: retrospective cohort study. BMJ. 2003;327:313.

4. Tocce KM, Sheeder JL, Teal SB. Rapid repeat pregnancy in adolescents: do immediate postpartum contraceptive implants make a difference? Am J Obstet Gynecol. 2012;206:481.e1-7.

5. Cea Soriano L, Garcia Rodriguez LA, Machlitt A, Wallander MA. Use of prescription contraceptive methods in the UK general population: a primary care study. BJOG. 2014;121:53-61.

6. Lunniss H, Cameron S, Chen ZE. Views of general practitioners on providing contraceptive advice and longacting reversible contraception at the 6-week postnatal visit: a qualitative study. J Fam Plann Reprod Health Care. 2016;42:99-106.

7. Ruiz-velasco V, Garcia C, Castro H. Cesarean section IUD insertion. Contracept Deliv Syst. 1982;3:21-4.

8. Chi IC, Zhou SW, Balogh S, NG K. Post-cesarean section insertion of intrauterine devices. Am J Public Health. 1984;74:1281-2.
9. Chi IC, Gao J, Siemens AJ, Waszak CS. IUD insertion at cesarean section - the Chinese experience. Adv Contracept. 1986;2:145-53.

10. Van Kets H, Thiery M, Van der Pas H. IUD insertion during cesarean section. Adv Contracept. 1985;1:337-9.

11. Puzey M. Mirena at caesarean section. Eur J Contracept Reprod Health Care. 2005;10:164-7.

12. Letti Müller AL, Lopes Ramos JG, Martins-Costa SH, Palma Dias RS, Valério EG, Hammes LS, et al.

Transvaginal ultrasonographic assessment of the expulsion rate of intrauterine devices inserted in the immediate postpartum period: a pilot study. Contraception. 2005;72:192-5.

13. Kapp N, Curtis KM. Intrauterine device insertion during the postpartum period: a systematic review. Contraception. 2009;80:327-36.

14. Celen S, Sucak A, Yildiz Y, Danisman N. Immediate postplacental insertion of an intrauterine contraceptive device during cesarean section. Contraception. 2011;84:240-3.

15. Goldstuck ND, Steyn PS. Intrauterine contraception after cesarean section and during lactation: a systematic review. Int J Womens Health. 2013;5:811-8.

16. Lester F, Kakaire O, Byamugisha J, Averbach S, Fortin J, Maurer R, et al. Intracesarean insertion of the Copper T380A versus 6 weeks postcesarean: a randomized clinical trial. Contraception. 2015;91:198-203.

17. Levi E, Canitllo E, Ades V, Banks E, Murthy A. Immediate postplacental IUD insertion at cesarean delivery: a prospective cohort study. Contraception. 2012;86:102-5.

18. Levi EE, Stuart GS, Zerden ML, Garrett JM, Bryant AG. Intrauterine device placement during cesarean delivery and continued use 6 months postpartum: a randomized controlled trial. Obstet Gynecol. 2015;126:5-11.

19. NHS Scotland Information Services Division. Births in Scottish Hospitals: year ending 31 March 2016. [Internet] Available online at: http://www.isdscotland.org/HealthTopics/Maternity-and-births/ (accessed April 17, 2017).

20. NHS Digital. Hospital Maternity Activity 2015-2016. [Internet] Available online at: http://www.content.digital. nhs.uk/catalogue/PUB22384 (accessed April 17, 2017).

21. Cameron S, Milne D, Galimore A, Craig A, Cowan S, Dundas K, et al. Feasibility and acceptability of introducing routine antenatal contraceptive counseling and provision of contraception after delivery: the APPLES pilot evaluation. BJOG. 2017 Apr 5. doi: 10.1111/1471-0528. 14674. [Epub ahead of print].

22. Faculty of Sexual and Reproductive Healthcare. UK Medical Eligibility Criteria for Contraceptive Use (UK MEC) 2016. [Internet] Available online at: http://ukmec.pa gelizard.com/2016 (accessed October 20, 2016).

23. The Scottish Government. Scottish Index of Multiple Deprivation. [Internet] Available online at: http:// www.gov.scot/Topics/Statistics/SIMD (accessed October 3, 2016). 
24. Faculty of Sexual and Reproductive Healthcare. Intrauterine Contraception Clinical Guidance. [Internet] Available online at: https://www.fsrh.org/standards-andguidance/documents/ceuguidanceintrauterinecontraception/ (accessed October 25, 2016).

25. Blumenthal PD, Goldthwaite LM. Intrauterine device insertion during cesarean delivery: the rising tide of the postdelivery intrauterine device. Obstet Gynecol.

2015;126:1-2.
26. Heinemann K, Reed S, Moehner S, Minh TD. Risk of uterine perforation with levonorgestrel-releasing and copper intrauterine devices in the European Active Surveillance Study on Intrauterine Devices. Contraception. 2015;91:274-9.

27. Faculty of Sexual and Reproductive Healthcare. FSRH Guideline: contraception after pregnancy. London: Faculty of Sexual and Reproductive Healthcare, 2017. 


\title{
Evaluating the attractiveness of the availability of injectable progestogen contraceptives at the community pharmacy setting in the United Kingdom
}

\author{
Rebecca Heller and Sharon T. Cameron \\ Chalmers Sexual \& Reproductive Health Centre, Edinburgh, UK
}

\section{Keywords}

community pharmacy; contraception; sexual health; skill mix

\section{Correspondence \\ Dr Rebecca Heller, Chalmers Sexual \& Reproductive Health Centre, 2A Chalmers Street, Edinburgh EH3 9ES, UK. \\ E-mail: rebecca.heller@ed.ac.uk}

Received May 22, 2015

Accepted January 3, 2016

doi: 10.1111/ijpp.12249

\begin{abstract}
Introduction Pharmacists have extended opening hours and are located in communities. Many offer sexual and reproductive health services such as emergency contraception. The opportunity to receive injectable contraception from community pharmacists would improve availability of this method and might increase uptake and continuation. A self-administered survey of women attending a large urban sexual and reproductive health clinic was undertaken to determine the acceptability of receiving contraceptive injections from a community pharmacist.

Methods Women aged 16-50 years attending an NHS walk-in sexual and reproductive health clinic were invited to complete questionnaires while they were waiting to attend an appointment with a clinician. Questionnaires asked women if they were current, previous or never users of the progestogen only injectable, their method of contraception and whether availability of the injectable from a local pharmacist would influence their decision to use this method.

Results Two hundred and forty questionnaires were distributed and 220 completed (92\%). A total of $9 \%$ of respondents were past users of the injectable $(n=21), 4 \%$ were current users $(n=8)$ and the remaining $87 \%$ were never users. Of those 191 current non-users, $33 \%(n=64)$ indicated that they would consider using this method if it was available at the pharmacy. The main perceived advantages of attending the pharmacy were quicker appointments (52\%) and easier access (47\%).

Conclusion Provision of the injectable contraceptive from a pharmacist might make this method attractive to almost one in three women who are not currently using it. This could be a strategy to improve uptake and continuation of

this method.
\end{abstract}

\section{Key Points}

- Almost one in three women surveyed who was not using the contraceptive injection would consider using it if they could get injections from their community pharmacy.

- Women felt that pharmacies would offer quicker appointments, easier access and better opening hours.

- This approach has the potential to improve uptake and continuation rates of this effective method of contraception.

\section{Introduction}

The contraceptive injection depot medroxyprogesterone acetate (DMPA) is given every 12 weeks, but discontinuation rates of this contraceptive method have been noted to be particularly high. ${ }^{[1-3]}$ Although not the only factor, remembering to return to clinic every 3 months, as well as the inconvenience of having to do so has been cited by users as a contributory factor to discontinuation. ${ }^{[1]} \mathrm{A}$ subcutaneous formulation of injectable contraception (Sayana Press ${ }^{\circledR}$, Pfizer Inc.) is now available in the UK. 
This preparation is a lower dose $(104 \mathrm{mg})$, but is similar in other aspects including efficacy, injection interval, return to fertility and bleeding pattern. ${ }^{[4]}$ The subcutaneous preparation comes packaged in a simple delivery system known as uniject, consisting of a polyethylene reservoir pre-filled with DMPA and an integral fine subcutaneous needle. This may facilitate administration of injectable contraception and so expand the range of health professionals who can administer it, including community pharmacists. Self-administration of subcutaneous DMPA has been shown to be feasible in several trials, ${ }^{[5-7]}$ and Sayana Press ${ }^{\circledR}$ has recently been licensed in the UK for this purpose. ${ }^{[8]}$

There is growing recognition of the potential that community pharmacists could play in delivering sexual health services within the UK. Pharmacies are located directly in communities, and have extended evening and weekend hours. Examples of sexual health services delivered successfully by community pharmacists include provision of emergency contraception (EC), ${ }^{[9,10]}$ and treatment of partners of individuals with uncomplicated Chlamydia infection. ${ }^{[11]}$ In addition, there have been pilot projects of provision of the combined oral contraceptive pill from the pharmacy, ${ }^{[12]}$ and pharmacists providing 'bridging contraception' (a 1 month supply of a progestogen-only pill) alongside oral EC. ${ }^{[13]} \mathrm{A}$ pharmacist in the UK has also been trained to fit contraceptive implants and give the DMPA-IM injection. ${ }^{[14]}$ Community pharmacists in the UK currently administer other subcutaneous injections and are essential for delivery of national flu vaccination programmes. ${ }^{[15-17]}$ Pharmacists administer these medications under a Patient Group Directive, a legal framework that allows certain registered health professionals to administer specified medicines to a pre-defined group of patients without the necessity of seeing a doctor. Patient Group Directives were first introduced through legislation in 2000, and are currently covered under The Human Medicines Regulation 2012. ${ }^{[18]}$ This questionnaire was administered in Scotland, where sexual health services differ somewhat at an administrative level from other parts of the UK. However, the same 'no cost' services to the public are provided (through the National Health Service) including contraceptive methods.

For women who are already established users of the contraceptive injection, receiving repeat injections from their community pharmacy may be an attractive option. Administration of subcutaneous DMPA by community pharmacists (using a more complicated system of syringe and needle) has already been shown to be feasible in pilot studies conducted in the United States. ${ }^{[19,20]}$

We wanted to establish whether the option of receiving the injectable contraceptive from a community pharma- cist was acceptable to women, particularly in the case of those who are using less effective methods.

\section{Methods}

From 3 February to 11 July 2014, an anonymous, selfadministered questionnaire was offered to women attending a single-site walk-in clinic at an integrated sexual and reproductive health $(\mathrm{SRH})$ service located in the centre of Edinburgh, UK. The site was selected as it sees a high volume of patients and, as research is often conducted from this centre, reception staff are used to handing out research materials to patients. Women attend this walk-in clinic largely for contraception and sexually transmitted infection screening. Clinic reception staff were asked to hand out a questionnaire to all women of reproductive age (16-50 years) attending the clinic, to complete while they were waiting to be called to see the doctor or nurse. Women attending the termination of pregnancy service or the menopause clinic were not invited to participate. Women were asked to place completed questionnaires in a closed collection box.

Questionnaires were piloted with five service users and consisted of a brief written introduction that outlined the injectable method of contraception and introduced the idea that it might be possible to receive injections at a community pharmacy in the future. It was explained to women that the injection would be subcutaneous ('under the skin rather than into the muscle'). Questions required simple tick-box responses, with a space to expand upon responses if wished. Questionnaires asked women if they were current, previous or never users of the injectable, which method of contraception they were currently using, and whether availability of the injectable from a local pharmacist would influence their decision to use this method. They were also asked to select perceived advantages or disadvantages of receiving their contraception from the community pharmacy from a predefined list of options.

\section{Data analysis}

All data was entered into Microsoft Excel for analysis. All questionnaire responses were entered including those where not all questions had been answered. Responses to questions were expressed as numbers and percentages, with percentages rounded to the nearest integer. Questions were analysed separately, i.e. if a questionnaire was not fully answered, analysis was performed on the questions that were answered.

The local Quality Improvement Team for SRH approved the project. The scientific officer of the local 
ethics committee reviewed the project proposal and confirmed that ethical approval was not required.

\section{Results}

Two hundred and forty questionnaires were distributed and 220 were returned (91\%). One hundred and sixtythree were fully completed (74\%) and the remaining 57 were partially completed. Three only had information completed about the contraception being used by the participant, and 54 had answers completed stating whether they would attend the pharmacist for the contraceptive injection, but no reasons completed why/why not. During the study period, 1605 women attended appointments at the walk-in clinic. The number of women attending is likely to be smaller as some women may have attended more than once.

The main contraceptive methods being used by respondents are outlined in Table 1. As a percentage of 220 returned questionnaires, there were eight current users of the injectable in the women surveyed (4\%), 23 previous users $(10 \%)$ and 187 who had never used it $(85 \%)$. Of those who had never used it, $33(18 \%)$ had considered using it in the past but decided against it.

Of 23 previous users, the most common reason for having stopped the injectable was irregular bleeding, cited

Table 1 Contraceptive methods currently being used by respondents (for respondents using more than one method, most effective method being used)

\begin{tabular}{|c|c|c|}
\hline $\begin{array}{l}\text { Method in use at time of } \\
\text { completing questionnaire }\end{array}$ & $\begin{array}{l}n(\%) \\
n=220\end{array}$ & $\begin{array}{l}n \text { (\% of users of this } \\
\text { method) that would } \\
\text { consider a contraceptive } \\
\text { injection from a } \\
\text { community pharmacist } \\
n=65\end{array}$ \\
\hline $\begin{array}{l}\text { Combined oral contraception } \\
\text { (pill/patch/ring) }\end{array}$ & $60(27 \%)$ & $15(25 \%)$ \\
\hline Condoms & $43(20 \%)$ & $12(28 \%)$ \\
\hline Not using any contraception & $34(16 \%)$ & $8(24 \%)$ \\
\hline Contraceptive implant & $30(14 \%)$ & $16(53 \%)$ \\
\hline $\begin{array}{l}\text { IUD (intratuterine } \\
\text { device)/Mirena IUS } \\
\text { (intrauterine system) }\end{array}$ & $27(12 \%)$ & $11(41 \%)$ \\
\hline Progestogen only pill & $9(4 \%)$ & $2(22 \%)$ \\
\hline Current depo users & $8(4 \%)$ & $1(13 \%)$ \\
\hline Withdrawal method & $2(1 \%)$ & 0 \\
\hline $\begin{array}{l}\text { Contraception is not } \\
\text { necessary for me }\end{array}$ & $3(1 \%)$ & 0 \\
\hline Trying for a pregnancy & $1(0.5 \%)$ & 0 \\
\hline Diaphragm & $1(0.5 \%)$ & 0 \\
\hline Vasectomy & $1(0.5 \%)$ & 0 \\
\hline $\begin{array}{l}\text { Natural family planning } \\
\text { methods }\end{array}$ & $1(0.5 \%)$ & 0 \\
\hline
\end{tabular}

Table 2 Reasons for stopping the contraceptive injection, or deciding not to use it

\begin{tabular}{|c|c|}
\hline Reason & $\begin{array}{l}n(\text { no. of times ticked) } \\
\text { (\%) (percentage of } \\
\text { respondents selecting it*) }\end{array}$ \\
\hline \multicolumn{2}{|l|}{$\begin{array}{l}\text { Reasons for stopping (completed } \\
\text { by } 23 \text { ex-users of this method) }\end{array}$} \\
\hline Irregular bleeding & $10(44 \%)$ \\
\hline Weight gain & $6(26 \%)$ \\
\hline Acne & $6(26 \%)$ \\
\hline $\begin{array}{l}\text { Having to see a nurse or } \\
\text { doctor every } 12 \text { weeks }\end{array}$ & $6(26 \%)$ \\
\hline \multicolumn{2}{|c|}{$\begin{array}{l}\text { Reasons for deciding not to use the method (completed by } 33 \\
\text { women who had considered using the contraceptive injection } \\
\text { but decided not to) }\end{array}$} \\
\hline Worry about weight gain & $28(85 \%)$ \\
\hline $\begin{array}{l}\text { Having to see a doctor or nurse } \\
\text { every } 3 \text { months }\end{array}$ & $24(73 \%)$ \\
\hline $\begin{array}{l}\text { Don't want a method where } \\
\text { your period stops }\end{array}$ & $22(67 \%)$ \\
\hline Delay in return to fertility & $15(45 \%)$ \\
\hline Worry about bone health & $14(42 \%)$ \\
\hline Prefer something more long acting & $14(42 \%)$ \\
\hline Other people put me off & $9(27 \%)$ \\
\hline Not familiar with this method & $8(24 \%)$ \\
\hline
\end{tabular}

*Percentages add up to $>100$ as most respondents selected more than one option.

by 10 women (44\%). Six respondents (26\%) indicated that having to see a doctor or nurse every 3 months was a reason for stopping this contraceptive method. Other reasons are cited in Table 2. Of the 33 women who had considered using the contraceptive injection but decided against it, $24(73 \%)$ cited having to see a doctor or nurse every 3 months as one of the reasons they had decided not to use it. Other reasons are further detailed in Table 2.

Out of 212 current non-users, 65 (31\%) said they would consider using the contraceptive injection if they could get this from their local pharmacy. A total of 58\% of them $(n=37)$ were using less effective methods or no methods. The methods that all 65 were using are in Table 1. In response to why this would be a good option for them, most respondents ticked more than one option from a list. The main reasons were quicker appointments $(52 \%)$ and easier access (47\%) Further reasons are detailed in Table 3. Just one current contraceptive injection user said that they would be interested in getting future contraceptive injections from a community pharmacy.

One hundred and forty-three respondents (65\%) said that they would not consider using the contraceptive injection even if it could be given by a community pharmacist. The reasons largely related to the method itself 
Table 3 Perceived benefits and disadvantages of receiving contraceptive injections from a pharmacist in the community

Perceived disadvantages (completed by 143 respondents who would not choose this option)

\begin{tabular}{ll}
\hline & $\begin{array}{l}n \text { (no. of times ticked) } \\
(\%) \text { (percentage of } \\
\text { respondents selecting it*) }\end{array}$ \\
\hline $\begin{array}{l}\text { Perceived disadvantages } \\
\text { method ever }\end{array}$ & $53(37 \%)$ \\
$\begin{array}{l}\text { Other: 'want hormone free method' } \\
\text { Other reasons given }\end{array}$ & $32(22 \%)$ \\
$\begin{array}{l}\text { Prefer to see a nurse or doctor } \\
\text { every } 12 \text { weeks }\end{array}$ & $25(17 \%)$ \\
$\begin{array}{l}\text { Prefer coming to this clinic } \\
\text { Prefer an injection into the muscle } \\
\text { to under the skin }\end{array}$ & $7(8 \%)$ \\
Do not trust a pharmacist to give it & $4(3 \%)$ \\
Did not answer & $0(0 \%)$ \\
\hline
\end{tabular}

Perceived benefits (completed by 65 respondents who indicated interest in this service)

\begin{tabular}{ll}
\hline Perceived benefits & $\begin{array}{l}n \text { (no of times ticked) } \\
(\%) \text { (percentage of } \\
\text { respondents selecting it*) }\end{array}$ \\
\hline Better opening hours & $25(38 \%)$ \\
Easier to get to & $31(47 \%)$ \\
Closer to my home & $20(30 \%)$ \\
Quicker appointment & $34(52 \%)$ \\
Prefer an injection under the skin & $14(21 \%)$ \\
Did not answer & $17(26 \%)$ \\
\hline
\end{tabular}

*Percentages add up to $>100$ as most respondents selected more than one option. 'Other reasons given included 'scared of jags', 'out at sea for work so will not be able to get injection', 'Mirena is better option for me' and concerns about bone health and a delay in return to fertility. These all represented reasons why the method would not be considered, regardless of location of delivery.

rather than the place of delivery. Fifty-three (37\%) said that they did not wish to use this method at all, and 32 respondents $(22 \%)$ ticked 'other' and stated that they wanted a hormone free method. Only $11(8 \%)$ stated that they would prefer to see a nurse or doctor. Reasons are further outlined in Table 3. We did not include cost as a factor because in the United Kingdom; all contraception is free to users, as is seeking advice from a pharmacist, doctor or nurse. Obtaining contraception from a pharmacist would therefore represent no financial advantage or disadvantage to patients.

\section{Discussion}

Our survey suggests that almost one in three women who is not currently a user of the contraceptive injection would consider using this method if they could receive injections from their local pharmacy. Quicker appointments, better opening hours and the pharmacy being easier to get to were all stated as reasons why women thought this would be a good option for them.

Questions examined only what people said they might do in theory, so it is possible that this may not truly reflect the proportion of women who would choose to go to the pharmacist for contraceptive injections if this were a current option. We also captured only a small number of current users. This may be an indication that regular users of DMPA are choosing to attend their General Practitioner (GP) or practice nurse, rather than a SRH clinic. It may also reflect the local service policy of actively encouraging women who need a repeat injection to attend their local GP practice for this, so that SRH consultations can be prioritised for individuals with more complex sexual health needs. It does mean, however, that the survey findings cannot estimate what proportion of total current users would switch to go a pharmacy if this option was available. It would be worthwhile repeating this survey in a primary care setting to see if results differ. Since we relied on reception staff handing out questionnaires to women, only a small proportion of women that were eligible were handed questionnaires. We did not capture information on demographics of respondents. Due to busy clinics and a high workload reception, staff were unable to keep track of how many eligible women were not offered questionnaires. The list of possible advantages and disadvantages of receiving contraception at the pharmacy were created based on what we anticipated women's views would be and not on qualitative research with users.

Interestingly, a quarter of ex-users stated that having to visit a doctor or nurse every 3 months had been a reason that led to them stopping this method of contraception, suggesting that some women might continue using the contraceptive injection if they did not have to make a regular appointment with a doctor or nurse. In keeping with the existing literature, the main reason cited for stopping was problematic bleeding. ${ }^{[1-3]}$ Among the women who had given consideration to using the contraceptive injection in the past, but elected not to use it, almost three quarters of respondents stated that the fact that you had to see a doctor or nurse regularly had been a factor in them deciding not to use it. This gives a strong indication that removing this aspect of the contraceptive injection might make it a more attractive option to women who are considering using this as their method of contraception.

Although almost one in three women indicated that they would be interested in the contraceptive injection if the option to receive it from pharmacies was available, this may be an overestimation of the proportion of women choosing this if available. When asked about the 
reason they had decided not to use, or stopped using, the contraceptive injection, respondents mainly cited many reasons unrelated to delivery of the contraception, notably irregular bleeding and weight gain. As with all contraception, women's decision to use a method is likely to be influenced by a number of factors, and perceived disadvantages may be more or less relevant when weighed up against perceived advantages. Our survey does seem to suggest that availability at a pharmacy would be a perceived advantage to a fair proportion of women who are using less effective methods. Very few women stated that they preferred to see a doctor or nurse or attend a clinic.

This research captures patients' opinions on a novel potential option for delivering contraceptive injections in the community and demonstrates that a significant proportion of women would welcome the possibility of receiving this method from a community pharmacy. The availability of contraception injections from a local pharmacy would increase access for women and might make this longer acting method more attractive to a greater number of women, notably to women who are put off by the necessity of regular appointments with a doctor or nurse, who may opt instead for less effective methods. It may also mean that women might be less likely to discontinue the method once they start using it and this could potentially prevent more unintended pregnancies. Although a pilot study of pharmacists administering the contraceptive injectable in the US did not observe any difference in continuation rates of the method compared to standard practice, the study was a pilot of just 50 women randomised to clinic or pharmacy. ${ }^{[19]}$ The study did confirm that women found the pharmacy convenient, that pharmacists were respectful and overall women were very satisfied with receiving the injectable from this setting.

Studies indicate that both providers and women prefer a subcutaneous injection of DMPA delivered via Sayana Press ${ }^{\circledR}$, s uniject system to an intramuscular injection DMPA. ${ }^{[21,22]}$ While self-administration of the subcutaneous DMPA remains highly acceptable to women, ${ }^{[5-7]}$ the option of receiving injections from pharmacists nevertheless expands the options for increasing access to this method and may be particularly attractive to women who do not want to self-administer injections.

Our survey did not specify whether injections from the community pharmacy would be repeat or first injections of this method. It would make sense for a pilot project to explore continuation injections from the pharmacy, following method initiation from a clinician.

\section{Conclusions}

Our survey indicates that for a significant proportion of women who are not using the contraceptive injection, the ability to receive repeat injections at a local pharmacy would be an attractive prospect. This could be a simple strategy that might increase uptake and continuation rates of injectable contraception, and reduce unplanned pregnancies.

Further research piloting pharmacists as providers of this method of contraception is therefore warranted. Our survey did not specify whether injections from the community pharmacy would be repeat or first injections of this method. It would make sense for a pilot project to explore continuation injections from the pharmacy, following method initiation from a clinician.

\section{Declarations}

\section{Conflict of interest}

Dr Cameron receives investigator initiated research funding from Pfizer. This particular research project received no specific grant from any funding agency in the public, commercial or not-for-profit sectors.

\section{Funding}

This research received no specific grant from any funding agency in the public, commercial or not-for-profit sectors.

\section{Acknowledgements}

Thanks to the receptionists at Chalmers Centre for their assistance in handing out questionnaires.

\section{Authors' contributions}

$\mathrm{RH}$ designed questionnaire, piloting and collection, data entry, analysis of results, primary author of manuscript. SC gave the original questionnaire idea. All Authors state that they had complete access to the study data that support the publication.

\section{References}

1. Davidson AR et al. Injectable contraceptive discontinuation and subsequent unintended pregnancy

among low-income women.

Am J Public Health 1997; 87: $1532-$

1534.
2. Paul C et al. Depot medroxyprogesterone acetate. Patterns of use and reasons for discontinuation. Contraception 1997; 56: 209-214. 
3. Potter LS et al. Depot medroxyprogesterone acetate pioneers: a retrospective study at a North Carolina health department. Contraception 1997; 56: 305-312.

4. Faculty of Sexual and Reproductive Healthcare. New product review from the clinical effectiveness unit. Subcutaneous depot medroxyprogesterone acetate (Sayana Press ${ }^{\circledR}$ ). Faculty of Sexual and Reproductive Healthcare, 2013.

5. Cameron ST et al. Pilot study of home self-administration of subcutaneous depo-medroxyprogesterone acetate for contraception. Contraception 2012; 85: 458-464.

6. Prabhakaran S, Sweet A. Self-administration of subcutaneous depot medroxyprogesterone acetate for contraception: feasibility and acceptability. Contraception 2012; 85: 453457.

7. Williams $\mathrm{R}$ et al. Self-administration of subcutaneous depot medroxyprogesterone acetate by adolescent women. Contraception 2013; 88: 401407.

8. Faculty of Sexual and Reproductive Healthcare. CEU statement on selfadministration of Sayana Press ${ }^{\circledR}$. Faculty of Sexual and Reproductive Healthcare, 2015.

9. Black KI et al. Provision of emergency contraception: a pilot study comparing access through pharma- cies and clinical settings. Contraception 2008; 77: 181-185.

10. Marston $\mathrm{C}$ et al. Impact on contraceptive practice of making emergency hormonal contraception available over the counter in Great Britain: repeated cross sectional studies. BMJ 2005; 331: 271-276.

11. Cameron $\mathrm{S}$ et al. Expedited partner therapy for Chlamydia trachomatis at the community pharmacy. BJOG 2010; 117: 1074-1079.

12. Parsons $\mathrm{J}$ et al. Evaluation of a community pharmacy delivered oral contraception service. J Fam Plann Reprod Health Care 2013; 39: 97101.

13. Michie L et al. Pharmacy-based interventions for initiating effective contraception following the use of emergency contraception: a pilot study. Contraception 2014; 90: 447-453.

14. News Team. New specialist contraception service commissioned in Newcastle. Pharm J [serial online] 2011. http://www.pharmaceutical-journal. com/news-and-analysis/news/new-spe cialist-contraception-service-commissi oned-in-newcastle/11083246.article (accessed: 22 May 2015). Archived by WebCite ${ }^{\circledR}$ at http://www.webcitation.org/6YiIPwqvo

15. Hind $\mathrm{C}$ et al. Successful provision of influenza vaccine from a community pharmacy in Aberdeen. Pharm J 2004; 273: 194-196.
16. Brown D et al. Review of services provided by pharmacies that promote healthy living. Int J Clin Pharm 2012; 34: 399-409.

17. Anderson C, Thornley T. "It's easier in pharmacy": why some patients prefer to pay for flu jabs rather than use the National Health Service. BMC Health Serv Res 2014; 14: 35.

18. National Institute for Clinical Excellence (NICE). Patient Group Directions. NICE medicines practice guideline 2 2011. www.nice.org.uk/ guidance/mpg2 (accessed Wednesday 4th February 2015).

19. Picardo C, Ferreri S. Pharmacistadministered subcutaneous depot medroxyprogesterone acetate: a pilot randomized controlled trial. Contraception 2010; 82: 160-167.

20. Monastersky Maderas N, Landau S. Pharmacy and clinic partnerships to expand access to injectable contraception. $J$ Am Pharm Assoc 2007;47:527-531.

21. Burke HM et al. Observational study of the acceptability of Sayana ${ }^{\circledR}$ Press among intramuscular DMPA users in Uganda and Senegal. Contraception 2014; 89: 361-367.

22. Burke HM et al. Provider acceptability of Sayana ${ }^{\circledR}$ Press: results from community health workers and clinic-based providers in Uganda and Senegal. Contraception 2014; 89: 368373. 


\title{
Postpartum contraception: a missed
} opportunity to prevent unintended pregnancy and short inter-pregnancy intervals

\author{
Rebecca Heller, ${ }^{1}$ Sharon Cameron, ${ }^{2}$ Rosie Briggs, ${ }^{3}$ Norma Forson, ${ }^{4}$ \\ Anna Glasier ${ }^{5}$
}

${ }^{1}$ Clinical Research Fellow, Chalmers Sexual \& Reproductive Health Service, Edinburgh, UK ${ }^{2}$ Consultant Gynaecologist, Chalmers Sexual \& Reproductive Health Service, Edinburgh, UK ${ }^{3}$ Foundation Doctor, Midwife, Royal Sussex County Hospital, Brighton, UK

${ }^{4}$ Midwife, Chalmers Sexual \& Reproductive Health Service,

Edinburgh, UK

${ }^{5}$ Honorary Professor, Department of Obstetrics and Gynaecology, Royal Infirmary of Edinburgh, Edinburgh, UK

\section{Correspondence to} Dr Rebecca Heller, Chalmers Sexual \& Reproductive Health Service, 2A Chalmers Street, Edinburgh EH3 9ES, UK; rheller@staffmail.ed.ac.uk

Received 17 December 2014 Revised 19 August 2015 Accepted 4 November 2015 Published Online First 8 December 2015

\section{CrossMark}

\begin{abstract}
Background Women in the postpartum period need effective contraception. Unintended pregnancies soon after childbirth may lead to abortion or short inter-pregnancy intervals associated with adverse outcomes. Using databases for a 6-month period (September 2013-February 2014) we examined the proportion of women attending for abortion in Edinburgh, Scotland who had given birth in the preceding 12 months, and the proportion of women giving birth in this region after an inter-pregnancy interval of 12 months or less. We also surveyed 250 women prior to discharge from the same maternity service about their contraceptive intentions.
\end{abstract}

Results Some 75/1175 (6.4\%) attending for abortion had given birth within the preceding 12 months and 332/4713 (7.0\%) postpartum women gave birth following an inter-pregnancy interval of 12 months or less. When considering parous women, percentages were $13.3 \%$ and $13.9 \%$, respectively. The majority $(n=237,96.7 \%)$ of postpartum women were not planning another pregnancy within the year but only a minority ( $n=32,12.8 \%$ ) were planning on using longacting reversible contraception (LARC), namely the implant or intrauterine device. However, $42.8 \%$ ( $n=107)$ indicated that if the implant or intrauterine contraception could be inserted before they left hospital then they would choose these methods ( $p<0.0001)$.

Discussion Almost one in thirteen women in our population presenting for abortion or giving birth has conceived within 1 year of giving birth. Provision of LARC immediately postpartum appears to be an attractive option to mothers, and could be an important strategy to prevent unintended pregnancy and short inter-pregnancy intervals.

\section{INTRODUCTION}

The World Health Organization (WHO) recommends that birth to pregnancy
Key message points

- One in thirteen women presenting for abortion or giving birth has conceived within 1 year of giving birth.

- More than one in eight parous women presenting for abortion or giving birth have conceived within a year of giving birth.

- Just under half of women say that they would be likely to choose an intrauterine method or implant if these methods were available in the immediate postpartum period.

intervals be at least 2 years in order to optimise maternal and infant outcomes across both resource-poor and resource-rich countries. ${ }^{1}$ Data from the UK have shown that women with intervals between births of less than 12 months are at increased risk of obstetric complications, premature birth and neonatal death, even after the potentially confounding effect of maternal obstetric history has been taken into account. ${ }^{2}$ It is therefore essential that in the postpartum period there are as few barriers as possible to accessing effective contraception, particularly long-acting reversible contraceptive (LARC) methods such as intrauterine contraception and the contraceptive implant, since these are the most effective at preventing pregnancy. ${ }^{3} 4$

Guidelines from the National Institute for Health and Care Excellence (NICE) state that method and timing of contraception should be discussed in the first week postpartum, although they provide no guidance about the content of this 
discussion. $^{5}$ The Faculty of Sexual \& Reproductive Healthcare guidelines advise that time should be found in both the antenatal and postnatal period to discuss all forms of contraception. ${ }^{6}$ However, a large internet survey of UK mothers found widespread dissatisfaction with the timing and quality of contraceptive advice received postnatally. ${ }^{7} 8$ Although traditionally contraception is discussed at the 6 week general practitioner visit, fertility may have returned by this time for women who are not exclusively breastfeeding. ${ }^{9}$ There is evidence that $35-57 \%$ of mothers resume intercourse within 6 weeks postpartum, ${ }^{10} 11$ and that attendance for additional visits required to insert an intrauterine method or implant is poor at this time. ${ }^{12}$ Caring for a young baby, as well as fatigue and adapting to a period of change, may make attendance particularly challenging. At delivery, however, the mother is already in a healthcare setting with access to trained health professionals. Provision of implants and intrauterine contraception from the maternity service after childbirth could be convenient for women as they are already in a healthcare setting with skilled providers available. Although some maternity services in the UK currently provide contraceptive implants for some women before they are discharged home, ${ }^{13}{ }^{14}$ this is not universal across UK maternity services.

The Royal Infirmary of Edinburgh (RIE) is the main delivery unit for women in NHS Lothian (Edinburgh and the surrounding region), a district that had more than 9000 births in the year ending 31 March $2013 .^{15}$ In NHS Lothian in 2013, there were 2314 induced abortions. ${ }^{16}$ The RIE and the Chalmers Centre for Sexual and Reproductive Healthcare are the main providers of abortion in NHS Lothian. The aim of this study was to determine what proportion of women attending the abortion service had given birth in the preceding 12 months, and what proportion of women giving birth in NHS Lothian had given birth following a birth to pregnancy interval of 12 months or less. We also wished to determine views of postpartum mothers on future contraception and in particular on the theoretical acceptability of provision of intrauterine contraception and implants in the immediate postpartum period.

\section{METHODS}

We examined the computerised database for women presenting for abortion at RIE and Chalmers Centre, Edinburgh between 1 September 2013 and 28 February 2014 to determine if women had given birth in the preceding year. All women attending the abortion service have a routine ultrasound for gestational dating, and so we calculated the inter-pregnancy interval from the date of last childbirth to the estimated date of conception of the index pregnancy (abortion request). The date of conception was assumed to be 2 weeks after the last menstrual period (using the ultrasound gestational age).
We used the regional maternity computer database (Trak) to identify women who gave birth during the same study period in the region. We then gathered further data on anyone with an inter-pregnancy interval of 12 months or less, namely a previous birth within 21 months (we assumed a gestation of 9 months for the index pregnancy and added 12 months for an inter-birth interval to give 21 months). This method may underestimate the number of inter-pregnancy intervals less than 12 months, as it does not take account of shorter gestations associated with preterm birth. [For reference purposes, in 2012/2013 the percentage of babies born under 37 weeks was $5.3 \%$ (476/9050 births)]. ${ }^{15}$

Demographic data collected on women from abortion and maternity cohorts included age, parity and deprivation scores based upon postcode area of residence. Due to the different ways that the services managed data, women presenting for abortion were assigned deprivation scores (DEPCAT) based on 2001 census data about their postcode at the time of presentation, ${ }^{17}{ }^{18}$ but for maternity patients the deprivation measure assigned was the Scottish Index of Multiple Deprivation (SIMD) spread into populationweighted categories of roughly equal quintiles. ${ }^{19} 20$

An anonymous self-administered survey asking women their views on postnatal contraception and contraceptive intentions was conducted between 3 October 2013 and 31 January 2014 on postnatal wards and the Lothian Birth Centre (low risk delivery unit) in the RIE. Specifically, women were asked whether they would be likely to use a contraceptive implant or intrauterine contraception, if these methods were available in the immediate postpartum period, before discharge from the maternity service. Questionnaires were distributed by a research midwife. Women who spoke little English and who were without an interpreter or who had had poor pregnancy outcomes (e.g. stillbirth) were not given a questionnaire. The questionnaire consisted mainly of a series of simple tick box answers or a Likert scale (very likely to very unlikely) to indicate level of agreement with a range of statements on contraception. The questionnaire also provided free space for any comments women wished to make. Women were asked to place placed completed questionnaires in a closed box on the ward.

The local Quality Improvement Team for obstetrics and gynaecology approved both projects. The scientific officer of the local ethics committee reviewed the project proposal and confirmed that ethical approval was not required.

\section{Statistics}

The questionnaires were coded and the data were entered into Microsoft Excel. Statistical analysis was conducted using IBM Statistical Package for Social Sciences (SPSS) software V.18 (SPSS Inc., Chicago, IL, USA). Descriptive statistics were performed for 
demographics and groups were compared by Chi-squared $\left(\chi^{2}\right)$ test of significance. Statistical significance was taken as $p<0.05$.

\section{RESULTS}

Women presenting for abortion within 1 year of childbirth Over the study period, 1179 women attended requesting abortion. Of these, 75 (6.4\%) had given birth within the year preceding conception of this index pregnancy. Considering only the parous women $(n=563)$ who attended the clinic, $13.3 \%$ of women had given birth within the year preceding the index conception. The average gestation at the time of presentation for abortion was 52 days. The average number of days between childbirth and the start of the index pregnancy as determined by ultrasound was 193 (just over 6 months), with a minimum of 51 days (just over 7 weeks). The average age of women who had given birth in the previous year was 26 (17-37) years. Some $29.3 \%$ of women $(n=22)$ who attended for abortion within 1 year of giving birth were aged 30 years or older. Table 1 demonstrates their demographics and compares them to the demographics of all women presenting to the same abortion service during this period. Women who had given birth within 12 months were more likely to be deprived $(p=0.016)$ and to be aged 20-34 ( $p=0.021)$.

\section{Women giving birth who previously delivered within 1 year of pregnancy}

A total of 4713 women gave birth in the region over the time period. Three hundred and thirty-two women had given birth within the preceding

Table 1 Demographics of women attending for abortion who had given birth within 12 months of conception of index pregnancy compared with demographics of all women attending for abortion within same time period (September 2013-February 2014)

\begin{tabular}{|c|c|c|}
\hline Demographics & $\begin{array}{l}\text { Women who have } \\
\text { given birth within } \\
1 \text { year }(N=75) \\
{[n(\%)]}\end{array}$ & $\begin{array}{l}\text { All women } \\
\text { attending for } \\
\text { abortion }(N=1179) \\
\text { [n }(\%)]\end{array}$ \\
\hline \multicolumn{3}{|c|}{ Deprivation Category* $(p=0.016)$} \\
\hline 1-2 (affluent) & $14(18.7)$ & $193(16.4)$ \\
\hline 3-5 (intermediate) & $44(58.7)$ & $820(69.6)$ \\
\hline 6-7 (most deprived) & $17(22.7)$ & $146(12.6)$ \\
\hline Unknown & 0 & $20 \quad(1.7)$ \\
\hline \multicolumn{3}{|l|}{ Age (years) $(p=0.021)$} \\
\hline$<20$ & $8(10.6)$ & $221(18.7)$ \\
\hline $20-24$ & $24(32.0)$ & $362(30.7)$ \\
\hline $25-29$ & $21(28.0)$ & $266(22.6)$ \\
\hline $30-34$ & $17(22.7)$ & $161(13.7)$ \\
\hline $35-39$ & $5 \quad(6.7)$ & $106(9.0)$ \\
\hline $40+$ & 0 & $61 \quad(5.2)$ \\
\hline Unknown & 0 & $2(0.2)$ \\
\hline
\end{tabular}

*See McLoone and Boddy. ${ }^{18}$
21 months (representing a birth to pregnancy interval of less than a year), representing $7.0 \%$ of the total births. When considering only the 2393 parous women, the percentage was $13.9 \%$. The average time between births of this cohort was 17 months, with the shortest period being 9.5 months between births (in this case the index birth was significantly preterm). One hundred and eight women $(4.5 \%$ of parous women) had a birth to birth interval of 15 months or less, representing conception within 6 months of childbirth.

The average age of the women with a birth to pregnancy interval of 12 months or less was 29 years (see Table 2). $53.6 \%(n=178)$ were aged 30 years or older. Table 2 demonstrates the deprivation and ages of women in both categories. There was a statistically significant difference in the characteristics of women with a short birth to pregnancy interval and the general population of women giving birth. Women who had given birth within 12 months of their previous birth were more likely to come from areas of deprivation $(p=0.002)$ and tended to be younger $(p=0.020)$.

\section{Questionnaires}

A total of 300 questionnaires were handed out to 318 postpartum women, present in the maternity wards over the study period, representing a distribution rate of $95 \%$. The completion rate was $83 \%$ (250 completed). The reasons why 18 women did not receive a questionnaire included inability to speak English and poor pregnancy outcome. These judgments were made through discussion with the midwives looking after the women on the ward. Women may have also

Table 2 Demographics of women giving birth who had given birth within the preceding 21 months compared with demographics of all women giving birth within same time period (September 2013-February 2014)

\begin{tabular}{|c|c|c|}
\hline Demographics & $\begin{array}{l}\text { Women who have also } \\
\text { given birth within } \\
12 \text { months }(N=332) \\
\text { [n (\%)] }\end{array}$ & $\begin{array}{l}\text { All women } \\
\text { giving birth } \\
(N=4713) \\
{[n(\%)]}\end{array}$ \\
\hline \multicolumn{3}{|c|}{ SIMD Quintile* $(p=0.002)$} \\
\hline 4-5 (affluent) & $107(32.2)$ & $1927(40.9)$ \\
\hline 3 (intermediate) & $79(23.8)$ & $912(19.4)$ \\
\hline 1-2 (deprived) & $137(41.3)$ & $1715(36.4)$ \\
\hline Unknown & $9(2.7)$ & 159 (3.4) \\
\hline \multicolumn{3}{|c|}{ Age (years) $(p=0.020)$} \\
\hline$<20$ & $10(3.0)$ & $190(4.0)$ \\
\hline $20-24$ & $61(18.4)$ & $633(13.4)$ \\
\hline $25-29$ & $83(25.0)$ & $1165(24.7)$ \\
\hline $30-34$ & $105(31.6)$ & $1554(33.0)$ \\
\hline $35-39$ & $64(19.3)$ & $927(19.7)$ \\
\hline $40+$ & $9 \quad(2.7)$ & $244 \quad(5.2)$ \\
\hline
\end{tabular}

${ }^{*}$ The Scottish Government. ${ }^{19}$

SIMD, Scottish Index of Multiple Deprivation. 
been absent from the ward when researchers were distributing questionnaires.

Of those completing the questionnaires, 49\% $(n=121)$ had given birth before. The average age was $30(16-47)$ years. Twenty percent of women were from DEPCAT Categories 1-2 (affluent), 65.6\% were from Categories 3-5 (moderate deprivation) and 8\% were from Categories 6-7 (severely deprived). Some $(6.4 \%)$ did not provide their postcode. Of the 242 (97\%) women who answered the question about whether or not they could recall ever having a discussion antenatally with a health care provider about future contraception, 73 (30\%) women indicated that they had discussed contraception during the pregnancy. Of this group, 56 women $(77 \%)$ had found the discussion helpful.

The majority of the women (237/245, 96.7\%) stated that they were not planning a baby in the next year. Table 3 shows which methods of contraception women intended using.

Only $32(12.8 \%)$ women were planning on using the contraceptive implant or intrauterine contraception and $27(10.8 \%)$ were planning male or female sterilisation; 88 (35.2\%) women indicated that they did not know what contraception they would be using postnatally. Four percent $(n=10)$ of women indicated that contraception was not necessary for them. The most popular method that women were planning to use was condoms (see Table 3).

Of 239 women who answered the question about whether they would choose an intrauterine method if it could be inserted prior to leaving the hospital, 78 $(32.6 \%)$ described themselves as 'very likely' or 'quite likely' to choose this method. Of 241 women answering the same question about the likelihood of choosing the contraceptive implant if it could be fitted before leaving the hospital, 74 (30.7\%) said they

Table 3 Intentions for contraceptive use $(n=250)$

\begin{tabular}{lrr}
\hline Intentions & {$[\boldsymbol{n}(\%)]^{*}$} \\
\hline Don't know & $88(35.2)$ \\
Condoms & $71(28.4)$ \\
Progestogen-only pill & $28(11.2)$ \\
Combined oral contraceptive pill & $24 \quad(9.6)$ \\
Progestogen-only implant & 16 & $(6.4)$ \\
Intrauterine contraceptiont & 16 & $(6.4)$ \\
Progestogen-only injectable & 13 & $(5.2)$ \\
Male sterilisation & 14 & $(5.6)$ \\
Contraception not necessary & 10 & $(4)$ \\
Female sterilisation & 10 & $(4)$ \\
Lactational amenorrhoea & 7 & $(2.8)$ \\
Combined contraceptive patch or ring & 2 & $(0.8)$ \\
Question not answered & 5 & $(2)$ \\
\hline
\end{tabular}

${ }^{*}$ Numbers add up to more than $100 \%$ as some respondents ticked more than one answer. Intrauterine contraception includes both the copper intrauterine device and the levonorgestrel-releasing intrauterine system. would be 'very likely' or 'quite likely' to choose this. Combining the numbers of women likely to choose either intrauterine methods or implants gives a total of $107(42.8 \%)$ respondents who stated they would opt for one of these methods if insertion were available before leaving hospital. The difference in planned use of implant and intrauterine method and 'theoretical' use of the method if it was immediately available was statistically significantly $(p<0.0001)$. Only $12(4.8 \%)$ women who completed questionnaires were aged 20 years or under and six $(50 \%)$ indicated that they would be very likely or quite likely to choose a contraceptive implant if available before discharge home.

\section{DISCUSSION}

Almost one in thirteen women in our population presenting for abortion or giving birth have conceived within 1 year of giving birth. Of women who already have children, over one in eight requesting an abortion or giving birth conceived again within 1 year of their previous birth. Yet the survey suggests that the vast majority of postnatal women do not plan on having another pregnancy within the next year. Does this suggest that we are failing to meet the contraceptive need of women postpartum? It may well do. Most women had not discussed ongoing contraception during the pregnancy with a health professional and most were unsure about what method they would use. Although one in ten women were considering intrauterine contraception or implants, almost one in two women indicated that they would 'in theory' choose these methods if they could be provided before they left hospital. This interesting finding may reflect the convenience of this option, or the removal of important barriers that would otherwise deter them from choosing this method. Immediate postpartum provision of these methods could therefore be an important strategy to prevent unintended pregnancies for women, in the same way that immediate uptake of LARC post-abortion reduces a woman's risk of having another abortion in the next 2 years. ${ }^{21}$ There is also evidence that uptake of contraceptive implants in the early postpartum period reduces the risk of another pregnancy in young women in the next 12 months. ${ }^{22}{ }^{23}$ Although there has been some concern about the impact of early initiation of progestogen on breastfeeding, the available evidence is consistently reassuring and shows no adverse effects on lactation or infant growth. ${ }^{24}$

Women who attended for an abortion within 1 year of giving birth were more likely to be from areas of deprivation, as were women who gave birth to a baby that had been conceived within 1 year of childbirth. Nevertheless, both women who gave birth after a short inter-pregnancy interval and those attending for an abortion were from a wide age range and all socioeconomic groups, suggesting that all women, not only 
those from vulnerable groups (who are often the focus of targeted, contraceptive interventions), 23 25-28 could benefit from immediate access to implants and intrauterine contraception postpartum.

Although a significant proportion of women expressed a desire for immediate intrauterine contraception, this service is not usually available in the UK, with insertion usually taking place after 4 weeks postpartum. $^{29}$ There is, however, reasonable evidence from low- and middle-income countries that postplacental insertion of intrauterine contraception (within 10 minutes of placental expulsion) is safe and effective, ${ }^{30-33}$ although expulsion rates of between $7 \%$ and $25 \%$ have been reported. ${ }^{30-32}{ }^{34}$ Insertion immediately postpartum may also be easier since the cervix is dilated. Small studies also show that insertion at elective caesarean section may be a particularly beneficial option, with low expulsion rates of 0 $4 \% .{ }^{33} 3536$

A major limitation of our study is that the survey asked women about what contraceptive method they would theoretically choose: that is, they are open to the idea in theory, but this is not the same as evidence of acceptability. Addressing the question of whether women would choose LARC immediately postpartum and whether this would result in fewer unintended pregnancies and fewer short inter-pregnancy intervals will need further research. In addition, this study is unable to determine what proportion of the pregnancies following a short inter-pregnancy interval was intentional. Any intervention to improve uptake of LARC postpartum would ideally therefore contain an educative component which makes clear the risks of short inter-pregnancy intervals, as well as providing the means to prevent them. Finally, although our research was conducted in one of the largest maternity services and abortion services in the UK, our findings cannot be assumed to reflect others throughout the UK.

\section{CONCLUSIONS}

This study shows that short inter-pregnancy intervals are not uncommon, However, our survey data suggest that a significant proportion of women might be open to choosing one of the most effective methods of contraception (intrauterine contraception or the implant) in the immediate postpartum period, if this option was available. Provision and uptake of these methods at this time could in theory prevent more unintended pregnancies that currently end in abortion, miscarriage, ectopic pregnancies or short interpregnancy intervals. This would have important health benefits for women, their children and their future opportunities. Research is therefore necessary to progress strategies to provide LARC in the immediate postpartum period and to evaluate the acceptability and effectiveness of such strategies. This will necessitate both training of healthcare providers in maternity services to counsel and provide LARC to women postpartum, and funding to provide this service.

Acknowledgements The authors would like to thank Rob Elton for assistance with statistics and Neil Blackie for assistance with maternity data.

Competing interests None declared.

Ethics approval South-East Scotland Research Ethics Board.

Provenance and peer review Not commissioned; externally peer reviewed.

Data sharing statement Women who filled in questionnaires were also asked about what they thought would make it difficult to obtain contraception after delivery. These data were not included as they were not felt to be relevant to the main argument of the paper. These data are available by contacting the corresponding author.

\section{REFERENCES}

1 World Health Organization (WHO). Report of a WHO Technical Consultation on Birth Spacing. Technical Consultation. Geneva, Switzerland: World Health Organization, 2006.

2 Smith GCS, Pell JP, Dobbie R. Interpregnancy interval and risk of preterm birth and neonatal death: retrospective cohort study. BMJ 2003;327:c3967.

3 Winner B, Peipert JF, Zhao Q, et al. Effectiveness of long-acting reversible contraception. N Engl J Med 2012;366:1998-2007.

4 Trussell J. Contraceptive failure in the United States. Contraception 2011;83:397-404.

5 National Institute for Health and Clinical Excellence (NICE). Postnatal Care: Routine Postnatal Care of Women and Their Babies [CG37]. July 2006. http://www.nice.org.uk/guidance/ CG37 [accessed 4 December 2015].

6 Faculty of Sexual \& Reproductive Healthcare (FSRH). Postnatal Sexual and Reproductive Health. London, UK: FSRH, 2009.

7 Mumsnet/bpas survey shows gaps in contraception care for new mums. http://www.bpas.org/bpasknowledge.php? year $=2012$ \&npage $=0$ \&page $=81$ \&news $=543$ [accessed 26 August 2014].

8 McCance K, Cameron S. Midwives' experiences and views of giving postpartum contraceptive advice and providing long-acting reversible contraception: a qualitative study. J Fam Plann Reprod Health Care 2014;40:177-183.

9 Jackson E, Glasier A. Return of ovulation and menses in postpartum nonlactating women: a systematic review. Obstet Gynecol 2011;117:657-662.

10 Connolly A, Thorp J, Pahel L. Effects of pregnancy and childbirth on postpartum sexual function: a longitudinal prospective study. Int Urogynecol J 2005;16:263-267.

11 Murphy C, British Pregnancy Advisory Service. Sex and Contraception After Childbirth: Supporting Women's Choices in the Postnatal Period. London, UK: British Pregnancy Advisory Service, 2014.

12 Ogburn JA, Espey E, Stonehocker J. Barriers to intrauterine device insertion in postpartum women. Contraception 2005;72:426-429.

13 Vanthuyne A, Joyce G, Pittrof R. Comment on 'Midwives' experiences and views of giving postpartum contraceptive advice and providing long-acting reversible contraception: a qualitative study'. J Fam Plann Reprod Health Care 2014;40:313-314. 
14 Smith E, McLellan A. Comment on 'Midwives' experiences and views of giving postpartum contraceptive advice and long-acting reversible contraception: a qualitative study'. J Fam Plann Reprod Health Care 2014;40:312-313.

15 NHS Scotland Information Services Division. Births in Scottish Hospitals: Year ending 31st March 2013. Edinburgh, UK: iSD Scotland, 2014.

16 NHS Scotland Information Services Division. Abortion statistics. Edinburgh, UK: iSD Scotland, 2014.

17 Carstairs V, Morris R. Deprivation and health in Scotland. Aberdeen, UK: Aberdeen University Press, 1991.

18 McLoone P, Boddy FA. Deprivation and mortality in Scotland, 1981 and 1991. BMJ 1994;309:1465.

19 The Scottish Government. Scottish Index of Multiple Deprivation 2012. 2012. http:/simd.scotland.gov.uk/ publication-2012/ [accessed 9 October 2014].

20 Deprivation. Information Services Division Scotland. http:// www.isdscotland.org/Products-and-Services/GPD-Support/ Deprivation/ [accessed 9 October 2014].

21 Cameron S, Glasier A, Chen Z, et al. Effect of contraception provided at termination of pregnancy and incidence of subsequent termination of pregnancy. BJOG 2012;119:1074-1080.

22 Tocce KM, Sheeder JL, Teal SB. Rapid repeat pregnancy in adolescents: do immediate postpartum contraceptive implants make a difference? Obstet Gynecol 2012;206:481. e1-481.e7.

23 Lewis LN, Doherty DA, Hickey M, et al. Implanon as a contraceptive choice for teenage mothers: a comparison of contraceptive choices, acceptability and repeat pregnancy. Contraception 2010;81:421-426.

24 Cameron S. Postabortal and postpartum contraception. Best Pract Res Clin Obstet Gynaecol 2014;28:871-880.

25 Wilson EK, Samandari G, Koo HP, et al. Adolescent mothers' postpartum contraceptive use: a qualitative study. Perspect Sex Reprod Health 2011;43:230-237.
26 Wilson EK, Fowler CI, Koo HP. Postpartum contraceptive use among adolescent mothers in seven states. J Adolesc Health 2013;52:278-283.

27 Lopez L, Hiller J, Grimes D, et al. Education for contraceptive use by women after childbirth. Cochrane Database Syst Rev 2012;8:CD001863.

28 Stevens-Simon C, Kelly L, Kulick R. A village would be nice but...it takes a long-acting contraceptive to prevent repeat adolescent pregnancies. Am J Prev Med 2001;21:60-65.

29 Faculty of Sexual and Reproductive Health Care (FSRH). UK Medical Eligibility Criteria for Contraceptive Use (UKMEC 2009). 2009. http://www.fsrh.org/pdfs/UKMEC2009.pdf [accessed 12 December 2014].

30 Kapp N, Curtis KM. Intrauterine device insertion during the postpartum period: a systematic review. Contraception 2009;80:327-336.

31 Grimes DA, Lopez LM, Schulz KF, et al. Immediate post-partum insertion of intrauterine devices. Cochrane Database Syst Rev 2010;5:CD003036.

32 Chen BA, Hayes JL, Hohmann HL, et al. A randomized trial of postplacental compared to delayed insertion of the levonorgestrel-releasing intrauterine device after vaginal delivery. Contraception 2009;80:205.

33 Lara R, Sánchez R, Aznar R. Application of intrauterine device through the incision of the cesarean section. Ginecol Obstet Mex 1989;57:23-27.

34 Mwalwanda CS, Black KI. Immediate post-partum initiation of intrauterine contraception and implants: a review of the safety and guidelines for use. Aust N Z J Obstet Gynaecol 2013;53:331-337.

35 Chi IC, Ji G, Siemens AJ, et al. IUD insertion at cesarean section - the Chinese experience. Adv Contracept 1986;2:145-153.

36 Levi E, Cantillo E, Ades V, et al. Immediate postplacental IUD insertion at cesarean delivery: a prospective cohort study. Contraception 2012;86:102-105. 
Postpartum contraception: a missed opportunity to prevent unintended pregnancy and short inter-pregnancy intervals

Rebecca Heller, Sharon Cameron, Rosie Briggs, Norma Forson and Anna Glasier

J Fam Plann Reprod Health Care 2016 42: 93-98 originally published online December 8, 2015

doi: 10.1136/jfprhc-2014-101165

Updated information and services can be found at:

http://jfprhc.bmj.com/content/42/2/93

\section{Notes}

\section{These include:}

References This article cites 24 articles, 4 of which you can access for free at: http://jfprhc.bmj.com/content/42/2/93\#BIBL

\section{Email alerting} service box at the top right corner of the online article.

To request permissions go to:

http://group.bmj.com/group/rights-licensing/permissions

To order reprints go to:

http://journals.bmj.com/cgi/reprintform

To subscribe to BMJ go to:

http://group.bmj.com/subscribe/ 